\title{
Insight into chemical, biological, and physical processes in coastal waters from dissolved oxygen and inert gas tracers
}

by

Cara Charlotte Marie Manning

B.Sc. (Hons.), University of Victoria (2010)

Submitted in partial fulfillment of the requirements for the degree of

Doctor of Philosophy

at the

MASSACHUSETTS INSTITUTE OF TECHNOLOGY

and the

WOODS HOLE OCEANOGRAPHIC INSTITUTION

February 2017

(C) 2017 Cara Manning. All rights reserved.

The author hereby grants to MIT and WHOI permission to reproduce and to distribute publicly paper and electronic copies of this thesis document in whole or in part in any medium now known or hereafter created.

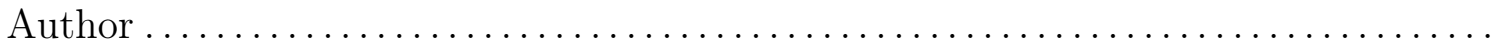

Joint Program in Oceanography and Applied Ocean Science \& Engineering Massachusetts Institute of Technology \& Woods Hole Oceanographic Institution

November 4, 2016

Certified by

Rachel H.R. Stanley

Assistant Professor, Department of Chemistry

Wellesley College

Thesis Supervisor

Certified by

David P. Nicholson Associate Scientist, Department of Marine Chemistry and Geochemistry Woods Hole Oceanographic Institution

Thesis Supervisor

Accepted by

Shuhei Ono

Chair, Joint Committee for Chemical Oceanography Massachusetts Institute of Technology Woods Hole Oceanographic Institution 


\title{
Insight into chemical, biological, and physical processes in coastal waters from dissolved oxygen and inert gas tracers
}

by

\author{
Cara Charlotte Marie Manning
}

\begin{abstract}
Submitted to the MIT-WHOI Joint Program in Oceanography and Applied Ocean Science and Engineering on November 4, 2016, in partial fulfillment of the requirements for the degree of Doctor of Philosophy in Chemical Oceanography
\end{abstract}

\begin{abstract}
In this thesis, I use coastal measurements of dissolved $\mathrm{O}_{2}$ and inert gases to provide insight into the chemical, biological, and physical processes that impact the oceanic cycles of carbon and dissolved gases. Dissolved $\mathrm{O}_{2}$ concentration and triple isotopic composition trace net and gross biological productivity. The saturation states of inert gases trace physical processes, such as air-water gas exchange, temperature change, and mixing, that affect all gases.

First, I developed a field-deployable system that measures $\mathrm{Ne}, \mathrm{Ar}, \mathrm{Kr}$, and $\mathrm{Xe}$ gas ratios in water. It has precision and accuracy of $1 \%$ or better, enables near-continuous measurements, and has much lower cost compared to existing laboratory-based methods. The system will increase the scientific community's access to use dissolved noble gases as environmental tracers.

Second, I measured $\mathrm{O}_{2}$ and five noble gases during a cruise in Monterey Bay, California. I developed a vertical model and found that accurately parameterizing bubble-mediated gas exchange was necessary to accurately simulate the He and Ne measurements. I present the first comparison of multiple gas tracer, incubation, and sediment trap-based productivity estimates in the coastal ocean. Net community production estimated from ${ }^{15} \mathrm{NO}_{3}{ }^{-}$uptake and $\mathrm{O}_{2} / \mathrm{Ar}$ gave equivalent results at steady state. Underway $\mathrm{O}_{2} / \mathrm{Ar}$ measurements revealed submesoscale variability that was not apparent from daily incubations.

Third, I quantified productivity by $\mathrm{O}_{2}$ mass balance and air-water gas exchange by dual tracer $\left({ }^{3} \mathrm{He} / \mathrm{SF}_{6}\right)$ release during ice melt in the Bras d'Or Lakes, a Canadian estuary. The gas transfer velocity at $>90 \%$ ice cover was $6 \%$ of the rate for nearly ice-free conditions. Rates of volumetric gross primary production were similar when the estuary was completely ice-covered and ice-free, and the ecosystem was on average net autotrophic during ice melt and net heterotrophic following ice melt. I present a method for incorporating the isotopic composition of $\mathrm{H}_{2} \mathrm{O}$ into the $\mathrm{O}_{2}$ isotope-based productivity calculations, which increases the estimated gross primary production in this study by $46-97 \%$.

In summary, I describe a new noble gas analysis system and apply $\mathrm{O}_{2}$ and inert gas observations in new ways to study chemical, biological, and physical processes in coastal waters.

Thesis Supervisor: Rachel H.R. Stanley

Title: Assistant Professor, Department of Chemistry

Wellesley College

Thesis Supervisor: David P. Nicholson

Title: Associate Scientist, Department of Marine Chemistry and Geochemistry

Woods Hole Oceanographic Institution
\end{abstract}




\section{Acknowledgments}

I am very grateful to my advisors, Rachel Stanley and Roo Nicholson, for their scientific mentorship. I am fortunate to have worked with not just one, but two wonderful advisors during my time at WHOI. My committee members, Carol Anne Clayson, Stephanie Dutkiewicz, and Bill Jenkins, as well as my thesis defense chair Mark Kurz, all deserve thanks for their contributions to my thesis. I wish to thank the other members of the Stanley lab and the WHOI Isotope Geochemistry Facility including Kevin Cahill, Josh Curtice, Joanne Goudreau, Evan Howard, Dempsey Lott, and Zoë Sandwith, who contributed their expertise to the laboratory and field work. I would also like to thank my coauthors and collaborators outside WHOI, especially Jason Smith who invited me to participate in the MBARI cruise which resulted in two chapters of my thesis. Many staff at WHOI including the Academic Programs Office staff, Marine Chemistry and Geochemistry department administrators, libraries staff, shipping and receiving staff, and security staff deserve kudos for helping WHOI to run so efficiently and assisting me at various points during my time at WHOI. I am grateful to Roberta Hamme, who introduced me to the world of gas tracers at the University of Victoria, and Karen Casciotti, who supervised me during my first summer at WHOI as a Summer Student Fellow, because I would not have found my way into graduate studies at MIT-WHOI without their mentorship. Finally, I would like to thank my family and friends (including all of the friends I have gained while at WHOI, as well as my friends back in Canada) for their support.

During my graduate studies, my research and studies have been supported by funding from the National Science Foundation (NSF) through grants OCE-8608400 and PLR1304406 (to RHR Stanley) and OCE-1129644 (to DP Nicholson), the WHOI Arctic Research

Initiative (funds to RHR Stanley and B Loose), a WHOI Innovative Technology grant (to RHR Stanley), the WHOI Coastal Ocean Institute (Student Research Fund to CC Manning), the WHOI Academic Programs Office (including an Ocean Ventures Fund grant to CC Manning), and the Houghton Fund at MIT. I received scholarships from the National Sciences and Engineering Research Council of Canada (NSERC) and the Canadian Meteorological and Oceanographic Society (CMOS). 


\section{Contents}

1 Introduction $\quad \mathbf{1 5}$

1.1 Background and motivation . . . . . . . . . . . . . . . 16

1.2 Tools for understanding the chemical, biological, and physical cycles of gases . 17

1.2 .1 Inert gas tracers . . . . . . . . . . . . . . . . . . . . 17

1.2.2 Deliberate gas tracer releases . . . . . . . . . . . . . . . . . . 19

1.2.3 Oxygen as a tracer of the biological pump . . . . . . . . . . . . . 19

1.2.4 Challenges in the use of gas tracers . . . . . . . . . . . . 20

1.3 Thesis overview . . . . . . . . . . . . . . . . . . . . . . . 21

1.3.1 A new field-deployable noble gas mass spectrometer - Chapter 2 . . . . 21

1.3.2 Evaluating published gas exchange parameterizations using a timeseries of noble gas measurements in Monterey Bay - Chapter 3 . . . . 22

1.3.3 Quantifying productivity by gas tracer, incubation, and sediment trap methods in Monterey Bay - Chapter 4 . . . . . . . . . . . . . 23

1.3.4 Productivity and gas exchange during seasonal ice melt in the Bras d'Or Lake - Chapter 5 . . . . . . . . . . . . . . . 24

1.4 Summary . . . . . . . . . . . . . . . . . . . . . 25

2 Continuous measurements of dissolved $\mathrm{Ne}, \mathrm{Ar}, \mathrm{Kr}$, and $\mathrm{Xe}$ ratios with a field-deployable gas equilibration mass spectrometer $\quad 27$

2.1 Abstract . . . . . . . . . . . . . . . . . . . . . . 28

2.2 Introduction . . . . . . . . . . . . . . . . . . 28

2.3 Experimental section . . . . . . . . . . . . . . . . . . . . . . . . . . . 29

2.3.1 Equilibration components . . . . . . . . . . . . . 30

2.3.2 Measurement components . . . . . . . . . . . . . . . . . . 32

2.3 .3 Data analysis . . . . . . . . . . . . . . . . . . . . . 34

2.4 Results and discussion . . . . . . . . . . . . . . . . . . 36

2.4 .1 Precision . . . . . . . . . . . . . . . 36

2.4 .2 Accuracy . . . . . . . . . . . . . . . . . . . 38

2.4 .3 Equilibration timescale . . . . . . . . . . . . . . . . . . . 42

2.4 .4 Pilot field study . . . . . . . . . . . . . . . . . . . . . . . 42

2.4.5 Comparison with other published methods . . . . . . . . . . . . . . 46

2.5 Conclusions . . . . . . . . . . . . . . . . . . . . . . 47 
2.6 Acknowledgments . . . . . . . . . . . . . . . . . . . . . 47

2.7 Supplemental information . . . . . . . . . . . . . . . . . . . 47

3 Quantifying air-sea gas exchange using noble gases in a coastal upwelling zone

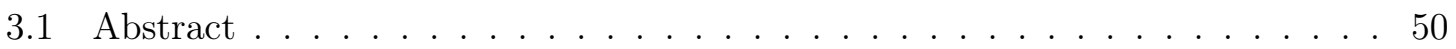

3.2 Introduction . . . . . . . . . . . . . . . . . 50

3.3 Methods . . . . . . . . . . . . . . . . . . . . . 51

3.3 .1 Cruise description . . . . . . . . . . . . . . . . . 51

3.3 .2 Noble gas data . . . . . . . . . . . . . . . . . . . . 52

3.4 Model description . . . . . . . . . . . . . . . . . . . . . . 53

3.4 .1 Data input and model setup . . . . . . . . . . . . . . . 53

3.4 .2 Adjustment for upwelling . . . . . . . . . . . . . . 55

3.4.3 Choice of gas exchange parameterizations . . . . . . . . . . . . . 57

3.5 Results . . . . . . . . . . . . . . . . . . . . . . . 59

3.6 Discussion . . . . . . . . . . . . . . . . . . . . . . . . 62

3.6.1 Modeled surface concentrations and saturation anomalies . . . . . . 62

3.6 .2 Modeled air-sea fluxes . . . . . . . . . . . . . . . . . . . . . . . 64

3.7 Conclusions and future work . . . . . . . . . . . . . . . . 66

3.8 Acknowledgments . . . . . . . . . . . . . . . . . . . . 67

3.9 Supplemental information . . . . . . . . . . . . . . . . . . 68

4 Impact of recently upwelled water on productivity investigated using in situ and incubation-based methods in Monterey Bay $\quad 69$

4.1 Abstract . . . . . . . . . . . . . . . . . . 70

4.2 Introduction . . . . . . . . . . . . . . . . . . 70

4.3 Background on methods . . . . . . . . . . . . . . . . . . 73

4.3.1 ${ }^{14} \mathrm{C}$ incubations . . . . . . . . . . . . . . . . 73

$4.3 .2{ }^{15} \mathrm{~N}$ incubations . . . . . . . . . . . . . . . . 73

$4.3 .3 \mathrm{O}_{2}$ mass balance . . . . . . . . . . . . . . . . . 74

4.3.4 Sediment traps . . . . . . . . . . . . . . . . . 75

4.4 Field and analytical methods . . . . . . . . . . . . . . 76

4.4 .1 Cruise description . . . . . . . . . . . . . . . 76

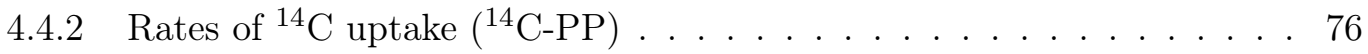

4.4.3 Rates of ${ }^{15} \mathrm{NO}_{3}^{-}$uptake, ${ }^{15} \mathrm{NH}_{4}^{+}$uptake, and nitrification . . . . . . . . 77

$4.4 .4\left[\mathrm{O}_{2}\right],[\mathrm{Ar}], \mathrm{O}_{2} / \mathrm{Ar}$, and ${ }^{17} \Delta$ analysis . . . . . . . . . 78

4.4.5 Sediment trap-based particle fluxes . . . . . . . . . . . . . 79

4.4 .6 Nutrients and pigments . . . . . . . . . . . . . . . . . . . . . 79

4.4 .7 Satellite data . . . . . . . . . . . . . . . . . . 80 
4.5 Calculations . . . . . . . . . . . . . . . . . . 80

4.5.1 Calculation of NOP from $\mathrm{O}_{2} / \mathrm{Ar}$ mass balance . . . . . . . . . . 80

4.5.2 Calculation of GOP from ${ }^{17} \Delta \ldots \ldots \ldots \ldots$

4.6 Results and discussion . . . . . . . . . . . . . . . . . . 85

4.6.1 Hydrographic and biogeochemical setting . . . . . . . . . . 85

$4.6 .2{ }^{14} \mathrm{C}$ incubations . . . . . . . . . . . . . . . . . . . . . . 89

$4.6 .3{ }^{15} \mathrm{~N}$ incubations . . . . . . . . . . . . . . . . . . . . . . . 89

$4.6 .4 \mathrm{NCP}$ and $\mathrm{NOP}$ from $\mathrm{O}_{2} / \mathrm{Ar} \ldots \ldots \ldots . \ldots . \ldots . \ldots 90$

4.6.5 GPP and GOP from ${ }^{17} \Delta \ldots \ldots \ldots . \ldots . \ldots . \ldots . \ldots 9$

4.6.6 Sediment trap-based carbon fluxes . . . . . . . . . . . . . . . 94

4.7 Synthesis and comparison with prior work . . . . . . . . . . . . 95

4.8 Implications and future directions . . . . . . . . . . . . . . . . . . . . . . . . 98

4.9 Acknowledgments . . . . . . . . . . . . . . . . . . . . . . 99

5 Changes in gross primary production, net community production, and air-water gas exchange during seasonal ice melt in the Bras d'Or Lake, a $\begin{array}{ll}\text { Canadian estuary } & 101\end{array}$

5.1 Abstract . . . . . . . . . . . . . . . . . . . . . . 102

5.2 Introduction . . . . . . . . . . . . . . . . . . . . . 102

5.3 Field work and analytical methods . . . . . . . . . . . . . . 105

5.3.1 Setup at Little Narrows . . . . . . . . . . . . . . . . . . . . 105

5.3 .2 Tracer injections . . . . . . . . . . . . . . . . . . . 107

5.3.3 Measurement of $\mathrm{O}_{2} / \mathrm{Ar}$ and the triple oxygen isotopic composition of $\mathrm{O}_{2} \ldots \ldots \ldots \ldots \ldots$. . . . . . . . . . . . . . . . . . . . . . . .

5.3 .4 Measurement of $\mathrm{SF}_{6} \ldots \ldots \ldots \ldots$. . . . . . . . . . . . . . . . . . . .

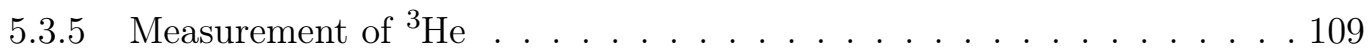

5.4 Calculations, results, and discussion . . . . . . . . . . . . . 109

5.4 Hydrography . . . . . . . . . . . . . . . . . . . . . . . . . . 109

5.4 .2 Gas transfer velocity . . . . . . . . . . . . . . . . . . 110

5.4 .3 Gross oxygen production . . . . . . . . . . . . . . . . 118

5.4 .4 Net oxygen production and export efficiency . . . . . . . . . . . . 128

5.4.5 Comparison to other productivity estimates . . . . . . . . . . 133

5.4.6 Effect of physical processes on productivity estimates . . . . . . . . 134

5.5 Conclusions . . . . . . . . . . . . . . . . . . . . 136

5.6 Acknowledgments . . . . . . . . . . . . . . . . . . . . 137

6 Conclusions and future directions $\quad 139$

6.1 Thesis summary . . . . . . . . . . . . . . . . . . . . . . 140

6.2 Future directions . . . . . . . . . . . . . . . . . . . 140 
6.2.1 Applications and modifications to the gas equilibration mass spectrometer . . . . . . . . . . . . . . . . . . . . . . . 140

6.2.2 Parameterization of air-sea exchange and other physical processes . . . 141

6.2.3 Lagrangian studies of gases and productivity . . . . . . . . . . . . . . 142

6.2.4 Characterization of the isotopic composition of photosynthetic $\mathrm{O}_{2}$. . 144

6.3 Broader implications . . . . . . . . . . . . . . . . . . . . . . 145

A Supplemental Information for Chapter 2: Continuous measurements of dissolved $\mathrm{Ne}, \mathrm{Ar}, \mathrm{Kr}$, and $\mathrm{Xe}$ ratios with a field-deployable gas equilibration mass spectrometer $\quad 149$

A.1 Introduction . . . . . . . . . . . . . . . . . . . . . 150

A.2 Alternative configurations tested . . . . . . . . . . . . . . . 150

A.3 Details on water pumps, dessicant, and membrane contactor . . . . . . . . . 153

A.3.1 Choice of water pumps . . . . . . . . . . . . . . . . . . . 153

A.3.2 Maintaining a dry environment in the headspace . . . . . . . . . . . 153

A.3.3 Potential permeation of $\mathrm{He}$ and $\mathrm{Ne}$. . . . . . . . . . . . . . . . . . . 154

A.4 Mass spectrometer settings _ . . . . . . . . . . . . . . . . . 155

A.4.1 Elimination of doubly-charged $\mathrm{CO}_{2} \ldots \ldots . \ldots . \ldots 156$

A.5 Field data . . . . . . . . . . . . . . . . . . . . . . . . . . 156

A.5.1 Data from Waquoit Bay, MA . . . . . . . . . . . . . . 156

A.5.2 Data on a moving vessel and the impact of vibration-induced noise . . 157

A.6 Other potential applications . . . . . . . . . . . . . . . . . . . . . . . . . 159

A.7 Parts list and photographs . . . . . . . . . . . . . . . . . . . . 161

B Supplemental Information for Chapter 3: Quantifying air-sea gas exchange $\begin{array}{lr}\text { using noble gases in a coastal upwelling zone } & 167\end{array}$

B.1 Software and data . . . . . . . . . . . . . . . . . . . . . . . 168

B.2 Description of figures . . . . . . . . . . . . . . . . 168

C Supplemental Information for Chapter 4: Impact of recently upwelled water on productivity investigated using in situ and incubation-based meth$\begin{array}{ll}\text { ods in Monterey Bay } & 171\end{array}$

C.1 Introduction . . . . . . . . . . . . . . . . . . . . . . . . . 172

C.2 Supplementary figures . . . . . . . . . . . . . . . . . . 173

D Supplemental Information for Chapter 5: Changes in gross primary production, net community production, and air-water gas exchange during seasonal ice melt in the Bras d'Or Lake, a Canadian estuary $\quad 179$

D.1 ADCP data . . . . . . . . . . . . . . . . . . . . . . . . 180

D.2 Photographs of study area . . . . . . . . . . . . . . . . 181 


\section{List of Figures}

$1-1 \quad$ Gas physical properties . . . . . . . . . . . . . . . . . . 18

2-1 Schematic of the GEMS setup . . . . . . . . . . . . . . . 30

2-2 GEMS precision determination . . . . . . . . . . . . . . . 37

2-3 GEMS accuracy determination . . . . . . . . . . . . . . . 41

2-4 GEMS equilibration timescale . . . . . . . . . . . . . . . . . 43

2-5 Measurements in Waquoit Bay, MA to evaluate the utility of the GEMS . . . 45

3-1 Map of the study site. . . . . . . . . . . . . . . . . . . . . . 52

3-2 Measured and idealized noble gas profiles . . . . . . . . . . . . 56

3-3 $\quad \mathrm{O}_{2}$, temperature, and $K_{z}$ profiles during the cruise $\ldots \ldots \ldots \ldots$

3-4 Near-surface gas concentrations and saturation anomalies . . . . . . . . . . 59

3-5 Model results without upwelling adjustment . . . . . . . . . . . . . . . . 62

3-6 Modeled air-sea gas fluxes of He and Xe . . . . . . . . . . . . . . . . . 64

4-1 Profiles of $\mathrm{K}_{z}, \mathrm{O}_{2}, \Delta\left(\mathrm{O}_{2} / \mathrm{Ar}\right)$, and ${ }^{17} \Delta \ldots \ldots \ldots \ldots$. . . . . . . 82

4-2 Map of study area and sea surface temperature . . . . . . . . . . . . . . 85

4-3 Profiles of temperature, $\mathrm{O}_{2}$, and $\mathrm{NO}_{3}^{-} \ldots \ldots \ldots \ldots$. . . . . 87

4-4 Near-surface measurements of temperature, $\mathrm{O}_{2}, \mathrm{NO}_{3}^{-}$, and $\mathrm{SiO}_{4} \ldots \ldots$. . . 88

4-5 Incubation and sediment trap results . . . . . . . . . . . . . . . . . . 91

4-6 GOP results and ${ }^{17} \Delta$ measurements . . . . . . . . . . . . . . . . . . . . 93

4-7 Energy flow diagram for Phase 1 and Phase $2 \ldots \ldots$. . . . . . . . . . 95

5-1 Map of Whycocomagh Bay and surrounding region . . . . . . . . . . . . . 104

5-2 Satellite images showing changes in ice cover in Whycocomagh Bay . . . . . . 111

5-3 Temperature and salinity profiles at Little Narrows . . . . . . . . . . . . . 112

5-4 Measured and modeled excess tracer ratios . . . . . . . . . . . . . . . 114

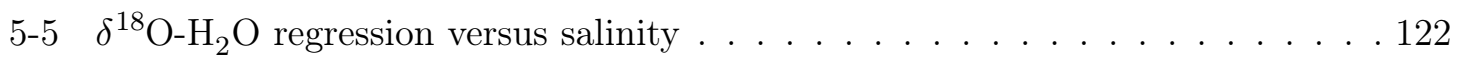

5-6 Gross oxygen production at Little Narrows . . . . . . . . . . . . . . . . . . . 125

5-7 Net oxygen production at Little Narrows . . . . . . . . . . . . . . . . . 130

A-1 Comparing performance of St707 and St2002 getter alloys . . . . . . . . 152

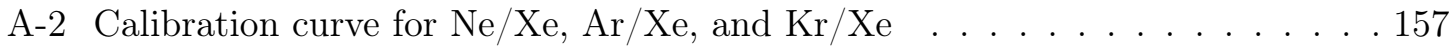

A-3 GEMS field data, calibrated only with air. . . . . . . . . . . 158 
A-4 GEMS field data, Xe ratios calibrated with discrete samples. . . . . . . . . 158

A-5 First photo of GEMS equilibration components . . . . . . . . . . . . . . 162

A-6 Second photo of GEMS equilibration components . . . . . . . . . . . . . . 163

A-7 Photo of GEMS measurement components . . . . . . . . . . . . . . . . . 164

A-8 Schematic of getter chambers . . . . . . . . . . . . . . . 165

B-1 Measured and idealized noble gas profiles, using published solubilities . . . . . 169

B-2 Near surface gas distributions, using published solubilities . . . . . . . . . . 169

B-3 Model results without upwelling adjustment, using published solubilities . . . 170

B-4 Modeled air-sea gas fluxes, using published solubilities . . . . . . . . . . . . 170

C-1 Underway shipboard measurements, all data . . . . . . . . . . . . . . . 173

C-2 Underway shipboard measurements from main study area . . . . . . . . . . 174

C-3 Modeled change in $\Delta\left(\mathrm{O}_{2} / \mathrm{Ar}\right)$ following non-steady state . . . . . . . . . 175

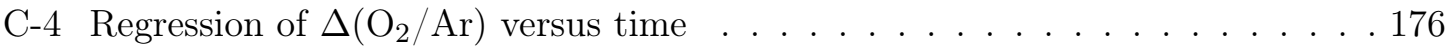

C-5 Underway temperature, salinity, and $\Delta\left(\mathrm{O}_{2} / \mathrm{Ar}\right) \ldots \ldots \ldots \ldots$

D-1 ADCP data from Little Narrows _ . . . . . . . . . . . . . . 180

D-2 Photo of Whycocomagh Bay on 28 March 2013 . . . . . . . . . . . . . . . 181

D-3 Photo of tracer injection on 30 March 2013 . . . . . . . . . . . . . . . 182

D-4 Photo of Whycocomagh Bay on 12 April $2013 \ldots \ldots$. . . . . . . . . . 183

D-5 Photo of Whycocomagh Bay on 12 April 2013 . . . . . . . . . . . . . . . 184 


\section{List of Tables}

3-1 Model performance of the four parameterizations . . . . . . . . . . . . . 61

3-2 Air-sea fluxes determined with different parameterizations . . . . . . . . . 66

4-1 Mixed layer productivity and nitrification results . . . . . . . . . . . . 90

5-1 Data for determination of the gas transfer velocity . . . . . . . . . . 115

A-1 Ion source settings f . . . . . . . . . . . . . . . . . 155

A-2 Acquisition settings for MID (multiple ion detection) experiment . . . . . . 156

A-3 Measurement components . . . . . . . . . . . . . . . . 161

A-4 Equilibration components . . . . . . . . . . . . . . . 166 
Chapter 1

\section{Introduction}




\subsection{Background and motivation}

The carbon cycle encompasses all of the mechanisms by which carbon is transferred between the atmosphere, ocean, land, and deep earth, and the environmental processes that convert carbon between different chemical forms. Carbon dioxide $\left(\mathrm{CO}_{2}\right)$ concentration in the atmosphere affects global climate because $\mathrm{CO}_{2}$ is a greenhouse gas. The deep ocean is an important regulator of atmospheric $\mathrm{CO}_{2}$ levels because it stores four times more carbon than the remainder of the biosphere combined (Ciais et al., 2013; Le Quéré et al., 2015). Glacialinterglacial changes in the atmospheric $\mathrm{CO}_{2}$ concentration are likely linked to changes in the exchange of $\mathrm{CO}_{2}$ between the deep ocean and atmosphere (Sigman and Boyle, 2000). This exchange occurs through a combination of physical processes (e.g., air-sea exchange and mixing) and biological and chemical processes (e.g., conversion of $\mathrm{CO}_{2}$ to organic carbon through photosynthesis and to solid $\mathrm{CaCO}_{3}$ through calcification). The rates of $\mathrm{CO}_{2}$ production and consumption (photosynthesis and respiration) are nearly balanced in the surface ocean, but a small fraction of the organic carbon produced sinks to the deep ocean, where it can be isolated from the atmosphere for timescales on the order of $1000 \mathrm{y}$ (Ciais et al., 2013).

Human activities are currently perturbing the natural carbon cycle. Emissions of $\mathrm{CO}_{2}$ from fossil fuel combustion, cement production, and land use change have caused the atmospheric $\mathrm{CO}_{2}$ concentration to increase by $40 \%$ over the past 250 years (Ciais et al., 2013; Le Quéré et al., 2015). To date, the oceans have absorbed 20-35 \% of total anthropogenic $\mathrm{CO}_{2}$ emissions (Sabine et al., 2004; Sarmiento and Gruber, 2006; Khatiwala et al., 2009; Wanninkhof et al., 2013; Ciais et al., 2013; Le Quéré et al., 2015).

There are currently large uncertainties in the rates of natural processes governing the exchange of carbon between the atmosphere and deep ocean, and these uncertainties make it difficult to determine how ocean carbon storage is changing now and may change in the future due to climate change. For example, recent estimates of the rate of organic carbon transport from the surface to the deep ocean range from $\sim 5-12 \mathrm{Pg} \mathrm{C} \mathrm{y}^{-1}$ (Laws et al., 2000; Henson et al., 2011; Siegel et al., 2016), similar to the rate of atmospheric emissions driven by human activities (currently $\sim 9-10 \mathrm{Pg} \mathrm{C}^{-1}$ ), and greater than the net flux of anthropogenic $\mathrm{CO}_{2}$ into the surface ocean $\left(\sim 2-3 \mathrm{Pg} \mathrm{C} \mathrm{y}^{-1}\right)$ (Ciais et al., 2013; Le Quéré et al., 2015). An improved understanding of both the natural carbon cycle and the anthropogenic influences on this cycle is needed to improve biogeochemical models of current and future climate.

Coastal regions play a disproportionately large role in the oceanic carbon cycle, relative to their small spatial extent (Cai et al., 2006; Cai, 2011; Bauer et al., 2013). Additionally, human activities strongly impact coastal regions (e.g., eutrophication, overfishing, and damming) and global warming is driving sea level rise, further altering coastal ecosystems (e.g., due to saltwater intrusion and erosion) (Cloern, 2001; Nicholls and Cazenave, 2010; 
Wong et al., 2014). There remains considerable debate regarding whether coastal waters are on average a net source or sink of $\mathrm{CO}_{2}$ and how climate change is affecting the magnitude and sign of coastal ocean carbon fluxes (Frankignoulle et al., 1998; Cai, 2011; Bauer et al., 2013). More observations are needed to resolve these uncertainties (Cai, 2011).

\subsection{Tools for understanding the chemical, biological, and phys- ical cycles of gases}

\subsubsection{Inert gas tracers}

Modeling $\mathrm{CO}_{2}$ and other biologically-active greenhouse gases is challenging, in part because the gases are simultaneously affected by biological, chemical, and physical processes. Furthermore, many of the physically-driven fluxes, such as air-sea gas exchange and diapycnal mixing, are challenging to measure directly. Some methods of estimating the physicallydriven fluxes such as eddy correlation measurements and turbulent energy dissipation measurements provide a snapshot of conditions over very small spatiotemporal scales (Gregg, 1987; Wüest et al., 1996; Fairall et al., 2000; Kunze et al., 2006). Gas tracers, in contrast, integrate over timescales of days to weeks in the surface ocean or years in the deep ocean and are a valuable tool for determining the average rates of these physical processes (Hamme and Emerson, 2002; Ito and Deutsch, 2006; Hamme and Severinghaus, 2007; Stanley and Jenkins, 2013), which are inherently patchy in space and time (Callies et al., 2015; Thompson et al., 2016).

Many investigators take the approach of measuring one or multiple inert gases, which are not biologically active, to parameterize the physical fluxes that affect all gases. Some inert gases, such as the stable and naturally-occurring noble gases $\mathrm{He}, \mathrm{Ne}, \mathrm{Ar}, \mathrm{Kr}$, and $\mathrm{Xe}$, are present throughout the world's oceans in concentrations near atmospheric equilibrium. The five stable noble gases have a wide range in molecular diffusivity, solubility, and temperature dependence of solubility (Wood and Caputi, 1966; Weiss and Kyser, 1978; Jähne et al., 1987a; Hamme and Emerson, 2004a), causing the saturation state of each gas to have a different sensitivity to a variety of physical processes (Stanley and Jenkins (2013), Figure 1-1).

Other inert gases exist solely because of human activities, such as the chlorofluorocarbons

(e.g., CFC-11 and CFC-12) and sulfur hexafluoride $\left(\mathrm{SF}_{6}\right)$. These gases are absorbed by the surface ocean and then mixed deeper into the water column through the oceanic thermohaline circulation (Fine, 2011). Furthermore, some naturally-present inert gases have concentrations and isotopic compositions that have been profoundly influenced by anthropogenic activities. For example, atmospheric nuclear weapons testing in the mid-20th century released ${ }^{3} \mathrm{H}$ (tritium) which is oxidized to water (i.e., $\left({ }^{3} \mathrm{H}_{1}\right)$ water) and slowly absorbed by the ocean and decays to ${ }^{3} \mathrm{He}$ with a $\sim 12.31$ y half life (Jenkins, 1998; MacMahon, 2006). The 

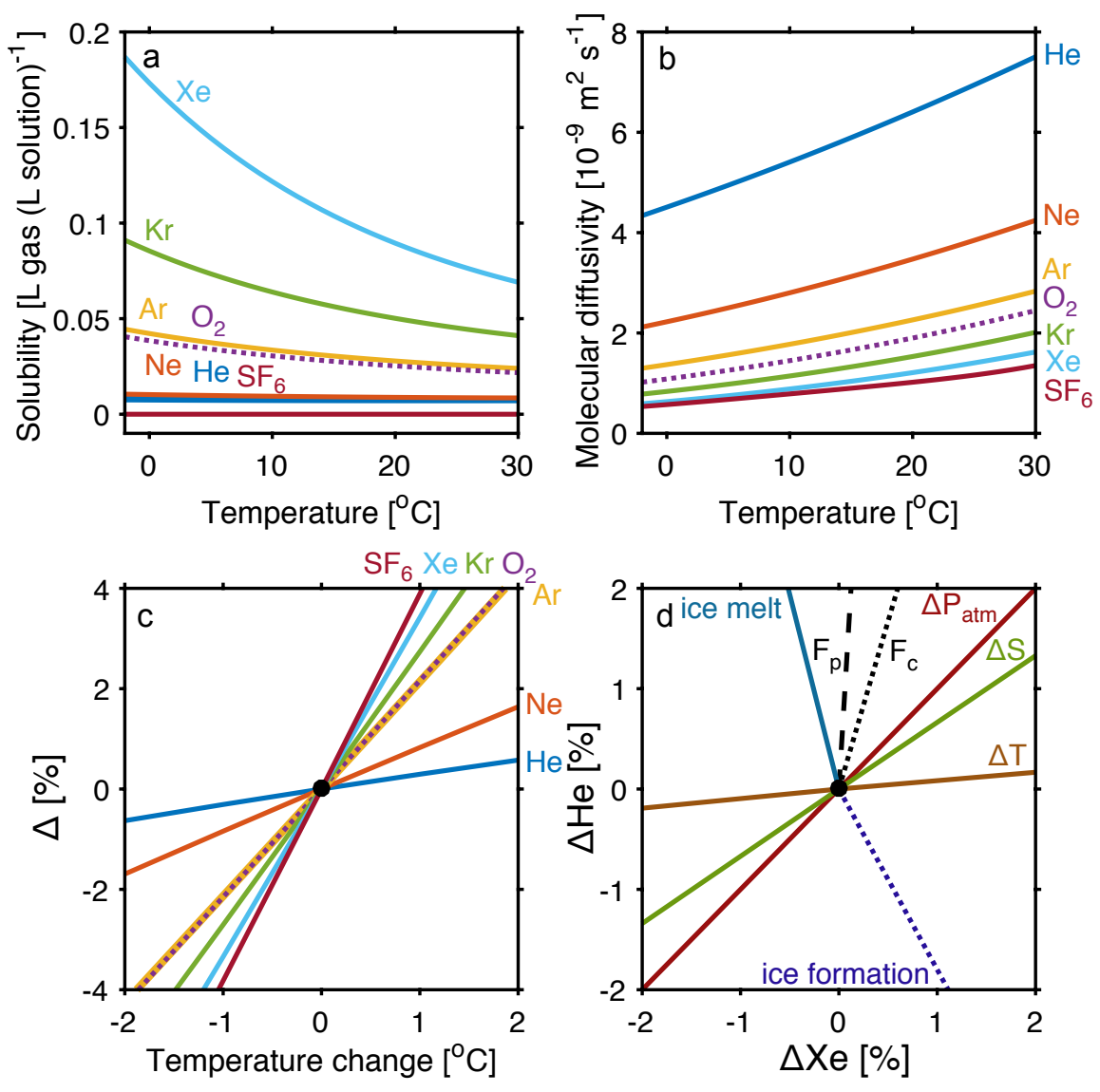

Figure 1-1: Differences in physical properties and response to physical forcing for gases studied in this thesis. The physical properties are (a) solubility in L of gas per L solution for a pressure of $1 \mathrm{~atm}$ of the pure gas (Bunsen solubility), and (b) molecular diffusivity $\left(\mathrm{m}^{2}\right.$ $\mathrm{s}^{-1}$ ). (c) Changes in gas saturation state that occur if a water mass initially at equilibrium with $\mathrm{S}=34$ PSS and $\mathrm{T}=10{ }^{\circ} \mathrm{C}$ is heated or cooled, without any air-sea gas exchange. Here $\Delta=\left(C / C_{e q}-1\right)$ where $C$ is the observed gas concentration and $C_{e q}$ is the gas concentration at saturation equilibrium. (d) Changes in He and Xe saturation state in response to specific physical processes when the gases are initially at equilibrium. The symbols $\Delta \mathrm{P}_{a t m}, \Delta \mathrm{S}$ and $\Delta \mathrm{T}$ refer to changes in atmospheric pressure, water salinity, and water temperature, respectively. The symbols $F_{c}$ and $F_{p}$ refer to the bubble-mediated air-sea flux due to completely dissolving bubbles and partially dissolving bubbles, respectively, and are calculated using the parameterization of Liang et al. (2013) and a wind speed of $u_{10}=$ $7 \mathrm{~m} \mathrm{~s}^{-1}$. The lines for ice melt and ice formation show the saturation anomalies generated by addition of glacial ice, as reported in Loose and Jenkins (2014) and formation of sea ice, as reported in Loose et al. (2016). References for solubility: He: Weiss (1971), Ne and Ar: Hamme and Emerson (2004a), Kr: Weiss and Kyser (1978), Xe: Wood and Caputi (1966) fit following the procedure in Hamme and Emerson (2004a), $\mathrm{O}_{2}$ : Garcia and Gordon (1992), and $\mathrm{SF}_{6}$ : Bullister et al. (2002). References for diffusivity: $\mathrm{He}, \mathrm{Ne}, \mathrm{Kr}$, Xe: Jähne et al. (1987a), Ar: extrapolated results from Jähne et al. (1987a), $\mathrm{O}_{2}$ : Ferrell and Himmelblau (1967), and $\mathrm{SF}_{6}$ : King and Saltzman (1995). The solubility and diffusivity of ${ }^{3} \mathrm{He}$ and ${ }^{4} \mathrm{He}$ are similar and therefore not plotted separately here. 
distributions of these gases within the surface and deeper ocean are interpreted in relation to their atmospheric histories to provide insights into the rates of physical processes affecting all gases (Jenkins, 1998; Bullister et al., 2006; Fine, 2011; Sonnerup et al., 2013).

\subsubsection{Deliberate gas tracer releases}

Other investigators pioneered the method of deliberate gas tracer releases to quantify air-sea gas exchange in the surface ocean and mixing processes in the deeper ocean (Ledwell et al., 1993; St. Laurent and Schmitt, 1999; Ledwell et al., 2000). In the gas exchange studies, investigators add one or more inert gases to the surface mixed layer, which are present at low ambient concentrations, and monitor how the gas concentrations or ratios evolve with time (Watson et al., 1991b; Wanninkhof et al., 1993). The rate of the change in the ratio can be used to calculate the gas transfer velocity. For gas exchange studies, the tracer pair should be chosen to have very different gas transfer velocities and a common choice is ${ }^{3} \mathrm{He}$ and $\mathrm{SF}_{6}$, which differ in diffusivity by a factor of 8 (Figure 1-1, Jähne et al. (1987a); King and Saltzman (1995)). These tracers also tag the surface water mass, enabling observations of the evolution of other biogeochemical parameters within the tracer patch (Watson et al., 1991a; Martin et al., 1994; Nightingale et al., 2000; Ho et al., 2011a).

\subsubsection{Oxygen as a tracer of the biological pump}

$\mathrm{O}_{2}$ is produced by photosynthesis and consumed by respiration concurrently with organic carbon production and consumption, respectively. Dissolved $\mathrm{O}_{2}$ is an effective tracer for net community $\mathrm{O}_{2}$ production, that is, the gross photosynthetic production of $\mathrm{O}_{2}$ by autotrophs minus community respiration by autotrophs and heterotrophs. A common approach is to use measurements of the $\mathrm{O}_{2} / \mathrm{Ar}$ ratio to quantify the biologically-driven supersaturation of $\mathrm{O}_{2}$; Ar and $\mathrm{O}_{2}$ respond similarly to physical forcing but Ar is biologically and chemically inert (Craig and Hayward, 1987; Emerson, 1987; Spitzer and Jenkins, 1989). At steady state, net community production should equal the rate of organic carbon export from the surface ocean (Laws, 1991).

Within the past 15 years, the triple oxygen isotopic composition of $\mathrm{O}_{2}$ has been established as a tracer of gross photosynthesis in the ocean due to the unique triple isotope signatures of $\mathrm{O}_{2}$ added by air-sea gas exchange, produced by photosynthesis, and consumed by respiration (Luz and Barkan, 2000, 2005; Juranek and Quay, 2013). Because the triple oxygen isotope tracer is relatively new, advances in our theoretical understanding of the tracer are ongoing (Kaiser, 2011; Luz and Barkan, 2011; Nicholson et al., 2011; Prokopenko et al., 2011), as are improvements in measurement precision and accuracy (Barkan and Luz, 2003, 2011; Stanley et al., 2010).

One advantage of $\mathrm{O}_{2}$-based tracers is that they integrate over larger spatial and temporal scales than other common productivity methods such as incubations and sediment traps 
(Juranek and Quay, 2005). Additionally, $\mathrm{O}_{2}$ can be measured at high frequency with sensors and mass spectrometers, enabling the detection of submesoscale variability in net community production and ocean carbon uptake (Tortell, 2005; Cassar et al., 2009; Tortell and Long, 2009).

\subsubsection{Challenges in the use of gas tracers}

A persistent challenge in the use of $\mathrm{O}_{2}$ and natural abundance noble gases as ocean tracers is that their concentrations are typically within a few percent of solubility equilibrium with the atmosphere (Hamme and Emerson, 2006; Hamme and Severinghaus, 2007; Stanley et al., 2009b; Nicholson et al., 2016). Insights into the rates of physical, chemical, and biological processes are revealed by these small deviations from equilibrium, and therefore the concentrations of these gases must be measured with high precision and accuracy. Additionally, the gas solubility and equilibrium isotope fractionation (if multiple isotopes are measured) as a function of temperature and salinity must be known very accurately (Hamme and Emerson, 2004a; Hamme and Severinghaus, 2007; Stanley et al., 2009b). Another challenge for many of the noble gases is that their concentrations are very low, typically less than $10 \mathrm{nmol} \mathrm{kg}-1$ for individual isotopes of $\mathrm{He}, \mathrm{Ne}, \mathrm{Kr}$, and Xe. Mass spectrometry is typically the method of choice for noble gas analysis because the gases are biologically and chemically inert, making reagent-based and optical methods infeasible. To date, published methods with sufficient precision and accuracy to resolve the small noble gas disequilibria observed in the ocean are generally laboratory-based, labor-intensive, and expensive (Severinghaus et al., 2003; Sano and Takahata, 2005; Hamme and Severinghaus, 2007; Stanley et al., 2009a; Nicholson et al., 2010; Aeschbach, 2016).

Precise and accurate measurements of $\mathrm{O}_{2}$ concentration are currently achieved a variety of ways including with chemical sensors and traditional wet chemistry methods (titrations) (Carpenter, 1965; Johnson et al., 2015; Bushinsky et al., 2016). In recent years, investigators have improved the stability and calibration procedures for $\mathrm{O}_{2}$ sensors so that they can operate autonomously for long deployments (Bushinsky and Emerson, 2013; Bittig and Körtzinger, 2015; Johnson et al., 2015; Bushinsky et al., 2016). However, regardless of the precision at which $\mathrm{O}_{2}$ is measured, it is still necessary to separately quantify the biological and physical fluxes of $\mathrm{O}_{2}$ in order to calculate the rate of net community $\mathrm{O}_{2}$ production. Inert gases are a valuable tool for developing parameterizations that are applicable to $\mathrm{O}_{2}$ because the gases span a range of solubility and diffusivity that envelops $\mathrm{O}_{2}$ (Figure 1-1 $\mathrm{a}-\mathrm{b})$.

Another challenge in the use of the gases as oceanic tracers is that many different processes are acting simultaneously, which makes interpretation of the data complex. For example, temperature change, diffusive air-sea exchange, bubble-mediated air-sea exchange, atmospheric pressure change, mixing between different water masses, ice freezing, and ice 
melt will all affect the saturation state and concentration of gases in surface waters (Figure 1-1 d). By measuring a suite of inert gases with a range of physiochemical properties it can be possible to disentangle the fluxes due to different processes, by modeling how each gas responds to different processes and finding the combination of processes that simulates all gas measurements most accurately. For example, Stanley et al. (2009b) and others show that time-series measurements of multiple inert gases can be used to separately quantify gas exchange due to diffusive exchange, complete bubble dissolution, and partial bubble dissolution (Spitzer and Jenkins, 1989; Hamme and Emerson, 2006; Stanley et al., 2006). Furthermore, measurements of multiple inert gases can be used to determine the relative proportions of different water masses in regions affected by glacial melt (Beaird et al., 2015) and water properties during deep-water formation and convection (Hamme and Severinghaus, 2007; Loose et al., 2016; Nicholson et al., 2016).

\subsection{Thesis overview}

The overall objective of my thesis is to improve understanding of coastal carbon, oxygen and inert gas cycles in coastal environments. The aims of my thesis are 1) to develop a new, field-deployable method for the measurement of natural abundance $\mathrm{Ne}$, $\mathrm{Ar}, \mathrm{Kr}$, and Xe ratios in water, and 2) to quantify air-sea gas exchange using inert gases and biological productivity using measurements of $\mathrm{O}_{2} / \mathrm{Ar}$ gas ratios and the triple isotopic composition of $\mathrm{O}_{2}$ in coastal waters, where the tracers have been underutilized to date.

\subsubsection{A new field-deployable noble gas mass spectrometer - Chapter 2}

Although noble gases show great promise as geochemical tracers, analytical capabilities have traditionally limited environmental applications of the full suite of noble gases and especially of the heavier gases $\mathrm{Kr}$ and Xe (Aeschbach, 2016). Within the past 15 years, several groups have published methods for measuring multiple noble gases including $\mathrm{Kr}$ and Xe with $0.6 \%$ precision or better (Sano and Takahata, 2005; Hamme and Severinghaus, 2007; Nicholson et al., 2010; Stanley et al., 2009a). However, these methods generally require the collection of discrete samples in the field and subsequent transport to a laboratory for time-consuming sample extraction and purification using cryogenic methods and vacuum lines. These instruments typically have low throughput and high cost, which has limited the number of groups who are able to use noble gases as geochemical tracers, and the spatiotemporal resolution of observations.

Other groups have developed compact mass spectrometer systems that are operable in the field and achieve precise, high-frequency measurements of more abundant dissolved gases such as $\mathrm{O}_{2}, \mathrm{~N}_{2}$, and Ar (Tortell, 2005; Cassar et al., 2009). These systems have dramatically expanded the oceanic data sets of $\mathrm{O}_{2}, \mathrm{Ar}$, and other gases as they obtain large quantities of 
data at relative ease compared to discrete sampling methods. Although portable methods for measuring the lower abundance noble gases were recently published (Machler et al., 2012; Visser et al., 2013), these methods do not obtain sufficient precision for oceanic applications.

In Chapter 2 (Manning et al., 2016a), I describe a new method for measuring Ne, Ar, $\mathrm{Kr}$, and $\mathrm{Xe}$ gas ratios in water using a field-deployable gas equilibration mass spectrometer. Compared to previously published methods, this new instrument has much higher throughput and lower cost, while also achieving precision and accuracy of $\sim 1 \%$, which is desirable for oceanic measurements (Hamme and Severinghaus, 2007; Ito et al., 2007; Stanley et al., 2009b; Nicholson et al., 2010, 2016).

I adapted the system from a published instrument, the equilibrator inlet mass spectrometer, which measures $\mathrm{O}_{2}$ and $\mathrm{Ar}$ (Cassar et al., 2009). I modified the system by adding

purification with nonevaporable getter alloys and changing the gas equilibration procedure to achieve full equilibration of the four noble gases. I validated the performance of the instrument in the lab and field and demonstrated its utility by using the system to continuously sample water from Waquoit Bay, Massachusetts over a 1-week period. This new instrument will enable more investigators to measure noble gases and use them as tracers of environmental processes (Aeschbach, 2016).

\subsubsection{Evaluating published gas exchange parameterizations using a time- series of noble gas measurements in Monterey Bay - Chapter 3}

The two mechanisms for air-sea gas exchange are diffusive exchange and bubble-mediated exchange. However, many gas exchange parameterizations do not explicitly include a bubblemediated flux and there is currently no consensus on the most accurate parameterization for bubble-mediated exchange (Liang et al., 2013; Emerson and Bushinsky, 2016; Plant et al., 2016). Published parameterizations predict a wide range of magnitudes for both the net bubble-mediated flux and the relative importance of bubbles that completely or partially dissolve for a given wind speed and gas saturation anomaly (Hamme and Emerson, 2006; Stanley et al., 2009b; Nicholson et al., 2011; Liang et al., 2013; Nicholson et al., 2016; Plant et al., 2016). For lower solubility gases, such as $\mathrm{O}_{2}$, a significant fraction of the total airwater flux is driven by bubbles, and uncertainties in the parameterization of bubble-mediated exchange directly translate to uncertainties in net community production calculated from $\mathrm{O}_{2}$ mass balance when simultaneous noble gas measurements are not available (Plant et al., 2016).

In Chapter 3 (Manning et al., 2016c), I use a six-day time-series of the five stable noble gases collected in Monterey Bay, California along with a one dimensional geochemical model to evaluate the performance of four different gas exchange parameterizations. The tested parameterizations include three that explicitly quantify the flux from partially and completely dissolving bubbles (Stanley et al., 2009b; Nicholson et al., 2011; Liang et al., 2013) 
and one that does not explicitly include bubble fluxes (Sweeney et al., 2007). I use profiles of temperature and salinity and diapycnal diffusivity collected at $6 \mathrm{~h}$ intervals throughout the cruise to force the model. My model demonstrates that all four parameterizations simulate the measurements of $\mathrm{Ar}, \mathrm{Kr}$, and Xe well; the saturation state of these gases was primarily driven by temperature change rather than air-sea gas exchange. For the lower solubility gases, $\mathrm{He}$ and $\mathrm{Ne}$, the models diverge and I find that the parameterization of Liang et al. (2013) simulates the He data the best. The He results are also very sensitive to the choice of solubility function, underscoring the need for accurate solubility functions for all five stable noble gases in seawater (Hamme and Severinghaus, 2007; Stanley et al., 2009b). These results demonstrate that gas exchange parameterizations developed for the open ocean may also be applicable to coastal environments (Ho et al., 2011a) and support other work demonstrating that time-series measurements of noble gases can be used to develop and evaluate gas exchange parameterizations explicitly including bubbles (Hamme and Emerson, 2006; Stanley et al., 2009b).

\subsubsection{Quantifying productivity by gas tracer, incubation, and sediment trap methods in Monterey Bay - Chapter 4}

Several methods are available for quantifying oceanic productivity and the rate of organic carbon export from the surface ocean to the deep ocean. All methods are thought to have inherent advantages and disadvantages, but often only one or two methods are used at one time, making it impossible to evaluate these biases. As the $\mathrm{O}_{2} / \mathrm{Ar}$ and triple oxygen isotope techniques have gained popularity, few published studies have directly compared incubationand gas-tracer based productivity estimates at the same time and location, and the methods most commonly compared, ${ }^{14} \mathrm{C}$ incubations and gas tracer measurements, do not measure the same aspects of the ecosystem metabolism (Halsey et al., 2013; Juranek and Quay, 2013). A second objective of the cruise described in Chapter 3 was to compare different methods of quantifying productivity.

In Chapter 4, I use measurements of $\mathrm{O}_{2}$ concentration and $\mathrm{O}_{2} / \mathrm{Ar}$ gas ratios, along with the triple oxygen isotopic composition of $\mathrm{O}_{2}$ to quantify net community production and gross primary production. I compare these gas tracer results with estimates of net community production and net primary production from incubations (measuring ${ }^{14} \mathrm{C}$ uptake, ${ }^{15} \mathrm{NO}_{3}^{-}$ uptake, ${ }^{15} \mathrm{NH}_{4}^{+}$uptake, and nitrification) and organic carbon export from sediment traps, to form a detailed picture of the ecosystem metabolism and carbon cycle state. To date, most

published studies simultaneously comparing net community production from ${ }^{15} \mathrm{NO}_{3}^{-}$uptake and from $\mathrm{O}_{2} / \mathrm{Ar}$ were conducted in high nitrate, low chlorophyll regions of the open ocean (Hamme et al., 2012; Giesbrecht et al., 2012) rather than nitrogen-limited, high productivity, coastal regions.

I show that at the start of the cruise (Phase 1) the methods agreed remarkably well. 
Net community production from $\mathrm{NO}_{3}^{-}$and from $\mathrm{O}_{2} / \mathrm{Ar}$ agreed within experimental uncer-

tainty despite the methods having very different measurement timescales and the ${ }^{15} \mathrm{~N}$ tracer concentrations added during the incubations being high relative to ambient concentrations (Dugdale and Goering, 1967). In the second half of the cruise (Phase 2), recently-upwelled filaments of water entered the study area and we sampled both within and outside of these filaments. Within the recently-upwelled water, incubations recorded higher productivity. Within the whole study area, continuous $\mathrm{O}_{2} / \mathrm{Ar}$ measurements reveal submesoscale variability that is not apparent from the once-daily incubations. These results demonstrate that incubation- and gas tracer-based estimates of productivity can give comparable results when the system is at steady state and highlight the advantages of using multiple productivity methods in a dynamic system.

\subsubsection{Productivity and gas exchange during seasonal ice melt in the Bras d'Or Lake - Chapter 5}

Temperatures in the Arctic are currently warming at two times the northern hemisphere rate due to anthropogenic climate change (ACIA, 2004; Bekryaev et al., 2010). Investigators agree that this warming is impacting oceanic biogeochemical cycles in this region, but there is not sufficient data to conclusively determine for the entire Arctic Ocean whether the biological pump is increasing or decreasing over decadal timescales and how its carbon uptake and storage may change in the future (Bates et al., 2006; Cai et al., 2010; Parmentier et al., 2013).

For example, the Arctic has undergone steady reductions in total sea ice cover as well as reductions in multi-year sea ice cover over the past three decades (since the beginning of the satellite record of sea ice) (Comiso et al., 2008; Maslanik et al., 2011), which has complex interactions with upper ocean properties and gas cycling. Sea ice blocks sunlight from entering the surface ocean and blocks gases from exchanging with the atmosphere, and thus the loss of sea ice will increase the amount of light available to phytoplankton within the upper ocean and increase the open water area for gas exchange (Parmentier et al., 2013). On the other hand, recent results have shown that sea ice itself can harbor algae that produce intense phytoplankton blooms, increasing regional estimates of primary production by up to an order of magnitude (Mundy et al., 2009; Arrigo et al., 2012). Furthermore, investigators have published conflicting results regarding the relationship between sea ice cover and gas exchange rates (Loose et al., 2011a; Rutgers van der Loeff et al., 2014; Lovely et al., 2015). One common approach is to assume that gas exchange scales linearly as a function of the fraction of open water (Takahashi et al., 2009; Evans et al., 2015), however, this assumption is not well-validated. Some investigators report enhanced gas exchange (Loose et al., 2009a; Else et al., 2011) or reduced gas exchange (Rutgers van der Loeff et al., 2014) in the presence of ice, relative to this linear relationship. 
Conducting field work in the Arctic is challenging due to its remoteness and the need for icebreakers to access many regions. As a result, measurements are seasonally and spatially biased, with many more observations in low-ice conditions during summer and fall than in high-ice conditions during winter and spring, and with more measurements on the continental shelves than in the open ocean (Parmentier et al., 2013).

In Chapter 5, I quantify productivity and air-sea gas exchange during seasonal ice melt in the Bras d'Or Lake, Nova Scotia, Canada, an estuary. I used this site as a natural laboratory to perform a controlled study of the processes affecting gas fluxes and productivity during ice melt. This study may shed light on similar processes occurring in the Arctic Ocean. We performed dual tracer release experiments (injection of ${ }^{3} \mathrm{He}$ and $\mathrm{SF}_{6}$ into the surface water) (Watson et al., 1991b; Wanninkhof et al., 1993) in a bay within the estuary and I quantified the air-sea gas exchange rate when the bay was nearly full of ice and when it was nearly ice-free. I also measured $\mathrm{O}_{2} / \mathrm{Ar}$ and the triple isotopic composition of $\mathrm{O}_{2}$ over a 1-month period as the bay transitioned from ice-covered to ice-free, and I use these data to quantify changes in net community production and gross primary production associated with the ice melt. We find that at $>90 \%$ ice cover, the gas transfer velocity is $94 \%$ lower than the velocity when the water is ice-free. I also present a new method for incorporating the local triple oxygen isotopic composition of $\mathrm{H}_{2} \mathrm{O}$, the substrate for photosynthetic $\mathrm{O}_{2}$, into the calculation of gross oxygen production from $\mathrm{O}_{2}$ triple isotope measurements.

\subsection{Summary}

As a whole, the body of work described in this thesis describes a new instrument for the environmental science community and advances knowledge of carbon, $\mathrm{O}_{2}$, and inert gas cycling in coastal and ice-covered waters. By developing a new field-deployable instrument that collects near-continuous measurements, I have expanded the types of scientific studies in which noble gases can be used as environmental tracers and the number of scientists who will be able to measure noble gases (Chapter 2). I have collected and applied gas tracer measurements at natural abundance, and through deliberate tracer releases, to quantitatively evaluate the performance of published gas exchange parameterizations at simulating measurements in a coastal region impacted by recently-upwelled water (Chapter 3), to compare productivity estimates from gas tracer, sediment trap, and incubation based-methods in a dynamic coastal region (Chapter 4), and to quantify the effects of changing ice cover on productivity and air-sea exchange in brackish waters (Chapter 5). In summary, the work in this thesis describes new ways that gases can be used to improve our knowledge of the chemical, biological, and physical processes affecting the carbon cycle in coastal regions, with a focus on air-water gas exchange and primary productivity. 


\section{Chapter 2}

\section{Continuous measurements of dissolved $\mathrm{Ne}, \mathrm{Ar}, \mathrm{Kr}$, and $\mathrm{Xe}$ ratios with a field-deployable gas equilibration mass spectrometer}

This chapter was originally published in Analytical Chemistry in 2016 and is reprinted with permission from the publisher, ACS Publications. Copyright 2016 American Chemical Society.

CC Manning, RHR Stanley, and DE Lott (2016) Continuous measurements of dissolved $\mathrm{Ne}, \mathrm{Ar}, \mathrm{Kr}$, and Xe ratios with a field-deployable gas equilibration mass spectrometer. Anal. Chem. 88 (6), 3040-3048. doi: 10.1021/acs.analchem.5b03102.

The supporting information for this chapter can be found in Appendix A. 


\section{$2.1 \quad$ Abstract}

Noble gases dissolved in natural waters are useful tracers for quantifying physical processes. Here, we describe a field-deployable gas equilibration mass spectrometer (GEMS) that provides continuous, real-time measurements of $\mathrm{Ne}, \mathrm{Ar}, \mathrm{Kr}$, and $\mathrm{Xe}$ mole ratios in natural waters. Gas is equilibrated with a membrane contactor cartridge and measured with a quadrupole mass spectrometer, after in-line purification with reactive metal alloy getters. We use an electron energy of $35 \mathrm{~V}$ for Ne to eliminate isobaric interferences, and a higher electron energy for the other gases to improve sensitivity. The precision is $0.7 \%$ or better and $1.0 \%$ or better for all mole ratios when the instrument is installed in a temperaturecontrolled environment and a variable-temperature environment, respectively. In the lab, the accuracy is $0.9 \%$ or better for all gas ratios using air as the only calibration standard. In the field (and/or at greater levels of disequilibrium), the accuracy is $0.7 \%$ or better for $\mathrm{Ne} / \mathrm{Kr}, \mathrm{Ne} / \mathrm{Ar}$, and $\mathrm{Ar} / \mathrm{Kr}$, and $2.5 \%$ or better for $\mathrm{Ne} / \mathrm{Xe}, \mathrm{Ar} / \mathrm{Xe}$, and $\mathrm{Kr} / \mathrm{Xe}$ using air as the only calibration standard. The field accuracy improves to $0.6 \%$ or better for $\mathrm{Ne} / \mathrm{Xe}, \mathrm{Ar} / \mathrm{Xe}$, and $\mathrm{Kr} / \mathrm{Xe}$ when the data is calibrated using discrete water samples run on a laboratory-based mass spectrometer. The e-folding response time is $90-410 \mathrm{~s}$. This instrument enables the collection of a large number of continuous, high-precision and accuracy noble gas measurements at substantially reduced cost and labor compared to laboratorybased methods.

\subsection{Introduction}

Noble gases are biologically and chemically inert, making them useful tracers of physical processes in the environment (Stanley and Jenkins, 2013). In water, measurements of dissolved noble gases in tandem with bioactive gases such as $\mathrm{O}_{2}$ can be used to separate the effects of biological versus physical processes on the equilibrium state of gases, enabling accurate estimates of biological productivity (Stanley et al., 2010; Nicholson et al., 2010; Stanley et al., 2006). Dissolved noble gas measurements can also be used to quantify oceanic processes such as gas ventilation in deep-water formation regions, diapycnal mixing, and sea ice melting and formation (Loose and Jenkins, 2014; Eveleth et al., 2014; Nicholson et al., 2010; Hamme and Severinghaus, 2007). On land, measurements of noble gases in groundwater can be used to generate paleotemperature records and for studies of groundwater-aquifer and groundwater-ocean interactions (Aeschbach-Hertig and Solomon, 2013; Castro et al., 1998; Stute and Schlosser, 1993).

Traditional methods for measuring multiple noble gases in natural waters via mass spectrometry involve the collection of discrete samples and laboratory-based analysis. Sample processing and analysis is time-consuming (often multiple hours per sample) and requires 
specialized and expensive equipment. Currently, very few labs in the world are capable of high-precision and high-accuracy (1\% or better) measurements of Ne, Kr, and Xe in natural waters, and oceanic measurements of dissolved noble gases are sparse, particularly for Xe (Loose and Jenkins, 2014; Hamme and Emerson, 2013; Stanley et al., 2009b; Hamme and Severinghaus, 2007; Nicholson et al., 2010).

Recently, the development of mass spectrometric methods for measurement of dissolved gases in the field(Machler et al., 2012; Cassar et al., 2009; Kameyama et al., 2009; Virkki et al., 1995) has led to high-resolution data sets of gases including $\mathrm{O}_{2}, \mathrm{Ar}, \mathrm{N}_{2} \mathrm{O}$ and dimethyl sulfide (Stanley et al., 2010; Marandino et al., 2009; Tortell, 2005). These instruments can analyze water in the field, in some cases eliminating the need to transport discrete samples back to the laboratory for subsequent analysis. In this paper we describe the gas equilibration mass spectrometer (GEMS), a new method for on-site measurement of $\mathrm{Ne}$, $\mathrm{Ar}, \mathrm{Kr}$, and $\mathrm{Xe}$ gas mole ratios in natural waters. We evaluate the precision and accuracy of the GEMS through comparison with a published laboratory-based method (Stanley et al., 2009a). Our relatively low cost ( $\sim 50000$ USD for the entire system in 2013) and low labor method will allow much higher throughput of noble gas measurement and will increase the number of scientists who are able to measure a suite of noble gases and use them as tracers for quantifying physical processes in the environment.

\subsection{Experimental section}

The GEMS can be separated into the equilibration components ('wet side'), and the measurement components ('dry side') (Figure 2-1). In brief, the equilibration components include the following features: filtered water is pumped through a membrane contactor cartridge containing a gas-permeable membrane, the headspace of the cartridge is continuously recirculated and dried, and gas is sampled via a capillary at a very low flow rate and transferred to the mass spectrometer. A switching valve is used to alternate between sampling from the cartridge and sampling ambient air, for calibration. The measurement components include metal alloy getters for purifying the gas stream, a quadrupole mass spectrometer, vacuum pumps, and a laptop computer. We describe below the final configuration that gave us the best results. We encourage scientists who are interested in building their own systems to consult the Supporting Information, where we describe some alternative configurations that were less effective. The Supporting Information also includes tables of instrument settings (Tables A-1-A-2) suppliers and part numbers (Tables A-3-A-4) and photos and schematics of the instrument (Figures A-5-A-8). 


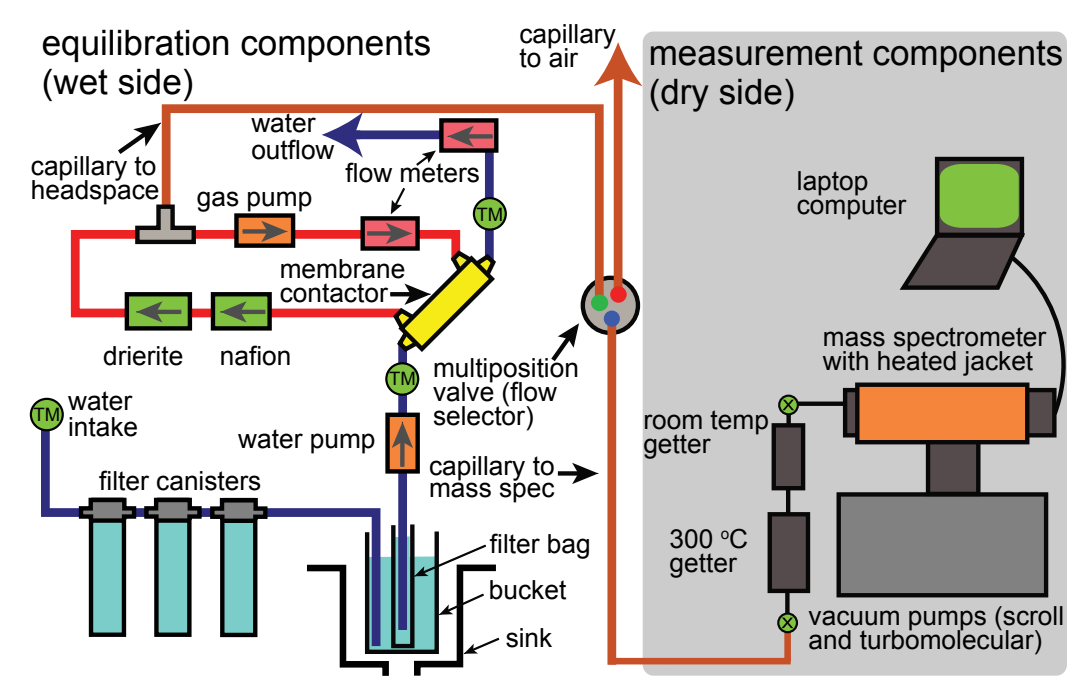

Figure 2-1: Schematic of the instrumental setup (not drawn to scale). See main text for description. See Figures A-5-A-7 for photos of the system.

\subsubsection{Equilibration components}

For shipboard installation, water from the ship's underway seawater line passes through three 10" filter canisters containing reusable pleated filters (100, 20, and $5 \mu \mathrm{m}$ nominal pore size) at a flow rate of $\sim 30 \mathrm{~cm}^{3} \mathrm{~s}^{-1}$ and then into a bucket placed in a sink (Figure 2-1). Alternatively, any natural water source, such as water from a groundwater well or lake, can be continuously pumped with a submersible well pump, filtered, and used to fill the bucket. A two-layer filter bag consisting of $100 \mu \mathrm{m}$ (outer) and $5 \mu \mathrm{m}$ (inner) nominal pore size felt is placed inside the bucket. The filters are necessary to prevent the membrane contactor from clogging. Flexible PVC tubing and a gear pump is used to transfer water at a flow rate of $\sim 18 \mathrm{~cm}^{3} \mathrm{~s}^{-1}$ from the filter bag to the membrane contactor and then to waste, down the sink drain (Figure 2-1). Our filter setup, water flow path, and gear pump is very similar to the configuration for the equilibrator inlet mass spectrometer developed by Cassar et al. (2009), except that we use the larger filter canisters to prolong the life of the disposable felt filter bags, due to our higher water flow rates. The filter canisters are not necessary when performing lab experiments with distilled or tap water.

The membrane contactor cartridge (Liqui-Cel Extra-Flow 2.5 x 8, model G540) contains hollow, tubular membranes composed of porous, hydrophobic polyethylene fiber. The tubes, called lumens, are $300 \mu \mathrm{m}$ diameter and the total membrane surface area is $1.4 \mathrm{~m}^{2}$. Water flows through the membrane contactor, outside the lumens, and gas dissolved in the water transfers across the lumens into the headspace (gas side) of the cartridge. Although liquid water does not cross the membrane, water vapor transfers through the pores and can condense on the headspace side of the membrane, reducing the gas transfer efficiency by clogging the pores (Lv et al., 2010; Wang et al., 2005). Therefore, the headspace is contin- 
uously recirculated and dried to improve gas transfer efficiency across the membrane. The headspace flows at $1.8 \mathrm{~cm}^{3} \mathrm{~s}^{-1}$ through a Nafion tube surrounded by molecular sieves and cobalt chloride indicator (PermaPure DM-110-24), then through a small piece of flexible PVC tubing containing $\sim 10$ g of indicating Drierite $\left(\mathrm{CaSO}_{4}, 10-20\right.$ mesh), then through a T-shaped fitting with a capillary adapter for sampling the gas, and finally through a diaphragm pump before re-entering the headspace (Figure 2-1). These drying techniques were selected because they do not require any additional gas or power sources. The headspace is recirculated in the opposite direction to the water, i.e., water enters and gas exits at the bottom of the cartridge. The recirculation loop increases the effective headspace volume by less than $10 \%$, and therefore it likely has a negligible effect on the response time. Without drying, the headspace partial pressure of water vapor $\left(p_{H 2 O}\right)$ is near saturation equilibrium, since water is observed to condense on the headspace side. With drying, $p_{H 2 O}$ in the headspace is somewhat lower and likely closer to ambient atmospheric $p_{H 2 O}$. We do not measure the gas humidity because, as discussed below, the vast majority of the water vapor is removed by the getters before entering the mass spectrometer.

A critical design principle of the gas equilibration mass spectrometer (GEMS) is that the gas in the headspace of the membrane contactor must be in equilibrium with the water flowing through the membrane contactor. If this condition is met, the gas mole ratios in water can be calculated from the measured headspace ratios, relative to air, and the gas solubility functions (Henry's law coefficients). At equilibrium, the headspace is composed of all the gases that are dissolved in the water, each at a partial pressure $(p)$ yielding equilibrium with the water flowing through the membrane contactor. This partial pressure of each gas can be calculated from its Henry's law coefficient, which is a function of the temperature and salinity of the water. If air-equilibrated water flows through the cartridge, the pressure of each gas in the headspace is equal to its pressure in air. If water that is 5 $\%$ supersaturated in Ne flows through the cartridge, the partial pressure of Ne will be $5 \%$ higher in the headspace compared to air. When first setting up the membrane contactor, we recommend allowing water to flow through the cartridge for at least $1 \mathrm{~h}$, to allow the headspace composition (which is initially air) to come into equilibrium with the water flowing through the cartridge.

To verify that the headspace is at a similar pressure to the ambient air, we temporarily placed a pressure measurement gauge (Convectron), calibrated to air, in the headspace recirculation loop. We found that the headspace pressure was within $\sim 1 \%$ of ambient air pressure when sampling air-equilibrated water.

To maintain equilibrium, we ensure that the rate of gas flow out of the headspace and into the mass spectrometer is negligible relative to the rate of gas transfer across the membrane. We use a long, small-diameter capillary $(0.05 \mathrm{~mm}$ ID, $5 \mathrm{~m}$ total length, deactivated fused silica) to achieve this low flow rate. The estimated gas flow rate through the capillary is $\sim 8$ 
$\times 10^{-5} \mathrm{~cm}^{3} \mathrm{~s}^{-1}\left(\sim 7 \mathrm{~cm}^{3} \mathrm{~d}^{-1}\right)$ based on a modified Hagen-Poiseuille equation Cassar et al. (2009) found that the Hagen-Poiseuille equation was a good approximation for capillary flow in a similar system. Calibration of the instrument is performed by periodically sampling air through a second capillary of the same dimensions.

Accurate measurement of temperatures throughout the water flow path is critical, in order to correct for the effects of changes in temperature on the saturation state of each gas. The temperature is measured at the water intake (using a sensor with accuracy of $\pm 0.05^{\circ} \mathrm{C}$ ), and immediately before and after the membrane contactor using two thermistors (accuracy $\pm 0.1^{\circ} \mathrm{C}$ ). The average of these two temperatures is used as the equilibration temperature. The thermistors (temperature sensors) are shown as green circles labeled TM in Figure 2-1. We reduce the magnitude of the temperature change by placing foam insulation around the filter canisters, tubing, and the membrane contactor.

\subsubsection{Measurement components}

A multiposition Valco valve is used to alternate between the two capillaries. The valve is connected to a $1 \mathrm{~m}$ long capillary to sample air, a $1 \mathrm{~m}$ long capillary to sample the headspace, and a common $4 \mathrm{~m}$ long capillary connected to the mass spectrometer. The valve to the mass spectrometer (Figure 2-1, blue circle) is always open, and the second open valve position switches from the headspace (red circle) to air (green circle) to perform a calibration. Sample gas flows from the membrane contactor (air), through the capillary and multiposition valve, through two chambers filled with reactive metal alloy getters and then into the ion source of the quadrupole mass spectrometer (Hiden HAL 3F RC201). Vacuum is provided by a combined turbomolecular and dry scroll pumping system (Agilent TPS-Compact). The pressure measured in the mass spectrometer is $\sim 1 \times 10^{-5} \mathrm{~Pa}$ while sampling air or the headspace of the membrane contactor.

Removal of unwanted gases such as $\mathrm{N}_{2}, \mathrm{O}_{2}$, and $\mathrm{H}_{2} \mathrm{O}$ from the gas stream greatly improves the detection limit by reducing molecule-molecule collisions within the mass spectrometer, and reduces matrix effects caused by differences in composition and pressure between the two gas streams. Published methods of noble gas analysis purify the gas stream using low temperature (cryogenic) traps and/or chemical purification (Stanley et al., 2009a; Sano and Takahata, 2005; Severinghaus et al., 2003; Visser et al., 2013). In-line purification with getters is ideal for a portable system because it does not require any additional maintenance in the field, nor the transport of cryogenic liquids. We used two custom-fabricated getter chambers (cylindrical stainless steel containers) filled with SAES Getters St2002 pellets (Figures A-7-A-8). During operation, the first can is heated to $300{ }^{\circ} \mathrm{C}$ and contains 100

$\mathrm{g}$ of getter; the second can is kept at room temperature and contains $30 \mathrm{~g}$ of getter. The heated getter breaks the $\mathrm{C}-\mathrm{H}$ bonds in $\mathrm{CH}_{4}$, and adsorbs all other gases except for $\mathrm{H}_{2}$ and the noble gases. The room temperature getter adsorbs $\mathrm{H}_{2}$, from pure $\mathrm{H}_{2}$ gas and from the 
decomposed $\mathrm{CH}_{4}$, and also adsorbs all the other gases, at a lower efficiency compared to the heated getter. We selected alloy St2002 due to its superior $\mathrm{N}_{2}$ removal efficiency (Figure A-1). Reactivation of the getter surface is performed by heating both chambers to $400{ }^{\circ} \mathrm{C}$ for $1 \mathrm{~h}$, and is required roughly once per month (when the signal intensity for $\mathrm{N}_{2}$ becomes greater than the signal intensity for ${ }^{40} \mathrm{Ar}$ ). The getter lasts approximately one year before replacement is needed. The temperature of both getter chambers is continuously recorded using thermocouples in contact with the heater elements. Using this purification method, $>98 \%$ of the non-noble gas content is removed from the gas stream before it enters the ion source, regardless of the initial gas content (total pressure, humidity, and abundance of other gases).

The noble gases are measured with a quadrupole mass spectrometer operated with a secondary electron multiplier (SEM) detector. The mole ratios are determined by selected ion monitoring. Calibration with air, which has known and constant noble gas mole ratios (COESA working group, 1976), is used to convert the averaged ion ratios to the deviation from saturation equilibrium. We measure $\mathrm{Ne}, \mathrm{Ar}, \mathrm{Kr}$, and Xe. Helium is not measured because we found that He permeates through the capillary and/or the cartridge (see Supporting Information), and $\mathrm{Rn}$ is not measured because its concentration is too low ( $\sim 6$ orders of magnitude less abundant than Xe in seawater).

The Hiden Analytical instrument was selected because it has the ability to measure individual selected ions at different electron energies, in a repeated sequence, without a loss in stability. We measure ${ }^{22} \mathrm{Ne}$ with a reduced electron energy to prevent formation of doubly charged $\mathrm{CO}_{2}$, which is a potential isobaric interference. Although the getters remove $>90 \%$ of the $\mathrm{CO}_{2}$, the signal intensity for $\mathrm{CO}_{2}$ after purification is sufficient to interfere with the ${ }^{22}$ Ne measurement. Therefore, we prevent the formation of the doubly-charged ion $\mathrm{CO}_{2}^{2+}$ by reducing the electron energy (cathode voltage) below $37 \mathrm{~V}$, as done by Hamme and Emerson (2004b) (see Supporting Information). We measure ${ }^{22} \mathrm{Ne}$ at an electron energy of $35 \mathrm{~V}$ and all other masses at an electron energy of $55 \mathrm{~V}$ (TablesA-1-A-2). The precision and sensitivity for $\mathrm{Ar}, \mathrm{Kr}$, and Xe is improved at the higher electron energy.

For Ar, we analyzed ${ }^{36} \mathrm{Ar}(0.337 \%$ abundance $)$ and $/$ or ${ }^{38} \mathrm{Ar}(0.0629 \%$ abundance $)$. The primary isotope, ${ }^{40} \mathrm{Ar}$, is 500-10 000 times more abundant in air than the other noble gases. The signal intensity for ${ }^{40} \mathrm{Ar}$ is too high to be read accurately using the SEM at our operating pressure (Visser et al., 2013). The signal intensities for ${ }^{36} \mathrm{Ar}$ and ${ }^{38} \mathrm{Ar}$ are at least 40 and 8 times higher, respectively, than the other noble gases we measure. In general, we found that measuring ${ }^{38} \mathrm{Ar}$ was preferred because it could be measured on the same amplifier as ${ }^{84} \mathrm{Kr}$, whereas ${ }^{36} \mathrm{Ar}$ had to be measured on a lower amplifier due to its higher abundance. By minimizing the range of signal intensities, we minimize nonlinearities in the detector response. For $\mathrm{Kr}$, the primary isotope, ${ }^{84} \mathrm{Kr}$ (57.0\% abundance) was chosen. For Xe, the least abundant gas, we measure both ${ }^{129} \mathrm{Xe}$ and ${ }^{132} \mathrm{Xe}$ (26.4 and $26.9 \%$ abundance, respectively), 
and take the average of the two signal intensities. Since Xe is the least abundant of the gases we measure, measuring both isotopes and taking the average reduces the noise compared to just measuring one isotope of Xe. Each measurement cycle (one measurement of each selected ion) takes $\sim 1 \mathrm{~min}$; see Tables A-1-A-2 for further details on the mass spectrometer settings.

We place a custom-fabricated heater jacket set to $50{ }^{\circ} \mathrm{C}$ around the manifold, to reduce the effects of room temperature change on the instrumental response, which is of particular concern when operating the instrument in the field, where there may be large fluctuations in ambient temperature. We use thermocouples to continuously monitor and record the room temperature and the manifold temperature. The mass spectrometer and vacuum pumps are connected to an uninterruptible power supply (UPS, Eaton 9130) to isolate them from power fluctuations.

The mass spectrometer data is acquired and saved using the manufacturer's software (MASsoft Pro 7). A custom Visual Basic program automates the valve switching between air and the headspace, and records temperatures and flow rates. The data from both programs is plotted in real time using Matlab.

The system described above was optimized for measurement of noble gas mole ratios. However, the equilibration components could potentially be used to equilibrate many other gases, given that we achieve full equilibrium of $\mathrm{Ne}$, Ar, $\mathrm{Kr}$, and $\mathrm{Xe}$, which span a factor of 10 range in solubility. We have successfully obtained high-accuracy, high-precision measurements of $\mathrm{O}_{2} / \mathrm{Ar}$ mole ratios using the system described above, with the getter chambers eliminated.

\subsubsection{Data analysis}

In this section, we describe how to use the raw mass spectrometer data (extracted ion profile) to calculate the mole ratios of the gases dissolved in water. As discussed above, if the rate of gas removal from the headspace by the capillary is negligible relative to the rate of gas transfer across the membrane, then full equilibration of the gases between the water flowing through the membrane contactor and the headspace can occur (Machler et al., 2012; Cassar et al., 2009).

We use the GEMS to determine the mole ratio of two gases (and the deviation of this ratio from equilibrium), rather than their individual concentrations (Cassar et al., 2009). If we recirculate air-equilibrated water through the membrane contactor, the measured ratios of any two noble gases are equivalent for the headspace versus air. However, the raw signal intensities for each gas in air versus the headspace are different by up to a few percent, and the magnitude of the offset can change with time. These offsets may be caused by slight differences in the rate of gas delivery to the mass spectrometer (e.g., due to differences in pressure between the headspace and air, or slight differences in the dimensions of the 
two capillaries), and/or differences in composition between the two gas streams causing matrix effects (Stanley et al., 2009a; Cassar et al., 2009). Although obtaining the individual concentrations would be ideal, the noble gas mole ratios can be effectively used to quantify physical processes (Hamme and Emerson, 2013; Nicholson et al., 2011, 2010).

We use Henry's Law to determine the equilibrium molality of any inert gas, such as $\mathrm{Ne}$

$$
n_{N e_{e q}}=p_{N e_{a i r}} \cdot H_{N e}(T, \text { salinity })
$$

where $n_{N e_{e q}}$ is the molar concentration dissolved in water at equilibrium $\left(\mathrm{mol} \mathrm{kg}^{-1}\right)$ and $p_{N e_{a i r}}$ is the partial pressure of Ne in dry air (atm). $H_{N e}$ is the Henry's Law solubility coefficient of $\mathrm{Ne}\left(\mathrm{mol} \mathrm{kg}^{-1} \mathrm{~atm}^{-1}\right)$ and is a function of the water temperature and salinity (Hamme and Emerson, 2004a; Benson and Krause Jr, 1976). We express the noble gas molar ratios in terms of the in situ deviation from the solubility equilibrium, often termed the saturation anomaly

$$
\Delta\left(\frac{N e}{X e}\right)=\left[\frac{\left(\frac{n_{N e}}{n_{X e}}\right)_{w}}{\left(\frac{n_{N e}}{n_{X e}}\right)_{e q}}-1\right] \times 100 \%
$$

where $\left(n_{N e} / n_{X e}\right)_{w}$ is the molar ratio of the gases dissolved in water and $\left(n_{N e} / n_{X e}\right)_{e q}$ is the molar ratio of the gases in the water at saturation equilibrium. Here, we show how the saturation anomaly, $\Delta(\mathrm{Ne} / \mathrm{Xe})$, can be determined from measurements of $(\mathrm{Ne} / \mathrm{Xe})_{h s}$ and $(\mathrm{Ne} / \mathrm{Xe})_{a i r}$, the ratios in the headspace and air, respectively. Following from Equation 2.1 the equilibrium gas ratio $(\mathrm{Ne} / \mathrm{Xe})_{e q}$ is defined as

$$
\left(\frac{N e}{X e}\right)_{e q}=\left(\frac{p_{N e}}{p_{X e}}\right)_{a i r}\left(\frac{H_{N e}}{H_{X e}}\right)_{T 1}
$$

where the subscript $T 1$ indicates the in situ temperature (where the water was sampled). For the membrane contactor, if the headspace is in equilibrium with the water passing through the cartridge, then we can calculate the ratio of the gases dissolved in water as

$$
\left(\frac{N e}{X e}\right)_{w}=\left(\frac{p_{N e}}{p_{X e}}\right)_{h s}\left(\frac{H_{N e}}{H_{X e}}\right)_{T 2}
$$

where the subscript $h s$ indicates the headspace and $T 2$ indicates the equilibration temperature inside the membrane contactor. By substituting Eqns. 2.3 and 2.4 into Equation 2.2, we find

$$
\Delta\left(\frac{N e}{X e}\right)=\left[\frac{\left(\frac{N e}{X e}\right)_{h s}\left(\frac{H_{N e}}{H_{X e}}\right)_{T 2}}{\left(\frac{N e}{X e}\right)_{a i r}\left(\frac{H_{N e}}{H_{X e}}\right)_{T 1}}-1\right] \times 100 \%
$$

Finally, using the definition of $\left(H_{N e} / H_{X e}\right)$ which follows from from Equation 2.1 at $T 1$ and 
T2, we obtain

$$
\Delta\left(\frac{N e}{X e}\right)=\left[\frac{\left(\frac{N e}{X e}\right)_{h s}\left(\frac{N e}{X e}\right)_{e q, T 2}}{\left(\frac{N e}{X e}\right)_{a i r}\left(\frac{N e}{X e}\right)_{e q, T 1}}-1\right] \times 100 \%
$$

Thus, the deviation of the gas ratios from solubility equilibrium can be determined by alternating between measurements of the noble gases in air and the headspace. The ratio in air is measured periodically (e.g., for a $40 \mathrm{~min}$ block after every 100-300 min of water sampling). We take the average of all the air measurements in each block (omitting the first and last $5 \mathrm{~min}$ ), and then apply a linear interpolation between each pair of air measurements to calculate the air ratio at the time of each headspace measurement, as done by Cassar et al. (2009) for $\mathrm{O}_{2} / \mathrm{Ar}$.

We measure $T 1 \mathrm{in} \mathrm{situ,} \mathrm{wherever} \mathrm{the} \mathrm{water} \mathrm{is} \mathrm{sampled.} \mathrm{For} \mathrm{example,} \mathrm{on} \mathrm{a} \mathrm{ship} T 1$ is measured using a sensor mounted on the hull of the ship adjacent to the seawater intake. T2 is determined from the average of two thermistors in the water flow path: one immediately before and one immediately after the membrane contactor. The salinity is measured once and we assume the in situ and equilibration salinities to be the same. We have observed $T 2$ to be up to $1.0{ }^{\circ} \mathrm{C}$ greater than $T 1$, which results in a $\sim 2.5 \%$ correction to the calculated $\Delta(\mathrm{Ne} / \mathrm{Xe})$ value.

\subsection{Results and discussion}

\subsubsection{Precision}

To determine the precision of the GEMS, we recirculated water from a temperature-controlled bath through the membrane contactor and collected data while continuously sampling from the headspace only. We then performed calculations to simulate the process of switching between air and the headspace (Figure 2-2). For these calculations, we used the ratios calculated from the raw signal intensity (extracted ion profile), without adjustment to the molar abundances in water or air. For example, the precision of the $\mathrm{Ne} / \mathrm{Kr}$ ratio was calculated from the signal intensity of ${ }^{22} \mathrm{Ne} /{ }^{84} \mathrm{Kr}$. We applied a linear interpolation to the raw ratio data, based on averaging 30 min of data every 340 min (i.e., simulating a 40 min measurement in air, with the first and last 5 minutes removed before averaging). This timing is identical to the timing of the lab-based accuracy experiment described below. A 7-min running mean filter was then applied to the 300-min intervals of data; this averaging time is equal to the e-folding response time of $\mathrm{Ne}$, which has the slowest response rate of the gases we measure. We define the precision as the relative standard deviation (RSD) of the difference between the filtered ratios and the interpolated ratios. In a temperature-controlled room, the precision is $0.7 \%$ or better for all gas ratios $(0.7,0.5,0.5,0.6,0.2$, and $0.6 \%$ for $\mathrm{Ne} / \mathrm{Xe}, \mathrm{Ne} / \mathrm{Kr}, \mathrm{Ne} / \mathrm{Ar}, \mathrm{Ar} / \mathrm{Xe}, \mathrm{Ar} / \mathrm{Kr}$ and $\mathrm{Kr} / \mathrm{Xe}$, respectively). We report the precision for 


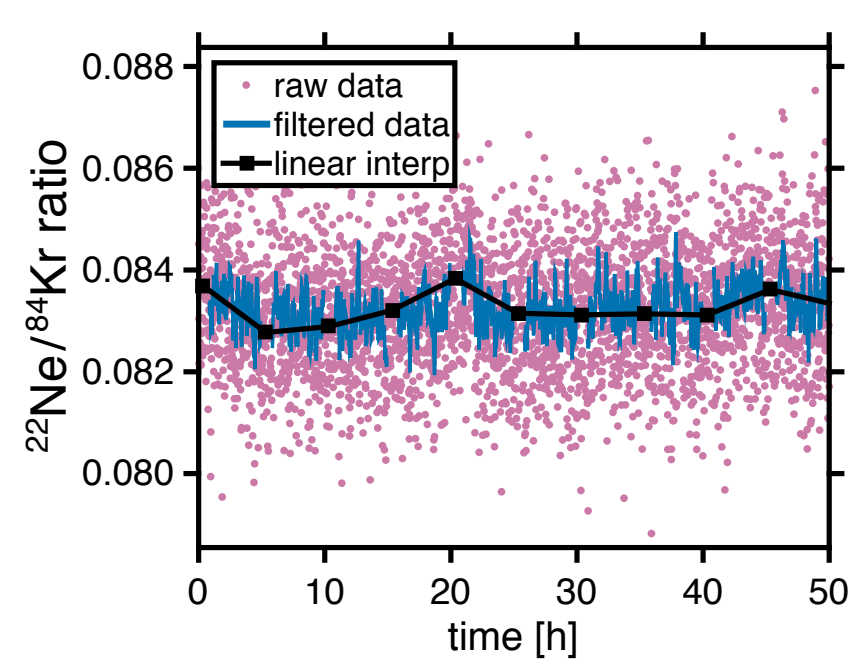

Figure 2-2: Measurements of ${ }^{22} \mathrm{Ne} /{ }^{84} \mathrm{Kr}$ signal intensity while sampling the headspace and recirculating air-equilibrated water through the membrane contactor. The pink dots show individual data points, the black line is a linear interpolation based on averaging $30 \mathrm{~min}$ of data every $340 \mathrm{~min}$, and the blue line is the data after applying a 7 -min running mean filter. The precision is calculated from the difference in magnitude between the blue and black lines.

all ratios as lighter gas/heavier gas for consistency. The relative precision (\% RSD) is the same for $\mathrm{Ne} / \mathrm{Xe}$ as for $\mathrm{Xe} / \mathrm{Ne}$. Because the different noble gases we measure have different abundances and physical properties, the gas ratios have varying precision and accuracy. We report the precision and accuracy for all gas combinations since the utility of each gas ratio to study environmental processes depends on the specific gases and on the precision/accuracy for that specific ratio. We got very similar results (precision of $0.7 \%$ or better for all gas ratios) when we measured air in the lab continuously, instead of water. When determining the precision from measurements of the headspace or air, we determine how similar each measurement is to the expected value. We did not alternate between measuring air and the headspace because if air was being used to calculate the expected headspace ratios, then any disequilibrium in the water with respect to air would bias the calculated precision.

In the field, the precision was somewhat worse, likely due to room temperature change. When we installed the mass spectrometer in an unheated garage, the mass spectrometer signal intensity for each ion drifted with temperature, despite the heater jacket on the manifold. In a variable-temperature environment, air calibrations should be performed more frequently. To determine the precision, we measured air continuously and then averaged 30 min of data every $150 \mathrm{~min}$. In this environment, the precision while measuring air was 1.0 $\%$ or better for all gas ratios $(1.0,0.6,0.5,0.9$, and $0.8 \%$ for $\mathrm{Ne} / \mathrm{Xe}, \mathrm{Ne} / \mathrm{Kr}, \mathrm{Ne} / \mathrm{Ar}, \mathrm{Ar} / \mathrm{Xe}$, $\mathrm{Ar} / \mathrm{Kr}$ and $\mathrm{Kr} / \mathrm{Xe}$, respectively). We did not determine the precision while measuring water in the field because we did not have access to a temperature-controlled water bath. 


\subsubsection{Accuracy}

To determine the accuracy of the GEMS, we compared the GEMS data to a published method (discrete samples analyzed by a laboratory-based mass spectrometer) during experiments in the field and the lab (Stanley et al., 2009a). In the lab, using the GEMS, we recirculated water from an insulated $0.12 \mathrm{~m}^{3}$ tank filled with distilled water that was open to the lab air. The water in the tank was constantly mixed using a submersible pump at the bottom of the tank. The temperature of the water was changed during the experiment. A filter sock was placed directly into the water bath and a gear pump connected to tubing was used to withdraw water from the filter sock and pump it through the membrane contactor. For the discrete samples, water was withdrawn using a spigot on the bath. Inside the tank, the spigot was connected to tubing, with the open end of the tubing placed next to the filter sock, so that the water removed for discrete sample collection would be near the water that entered the membrane contactor. Outside the tank, the other end of the spigot was connected to tubing for sampling. In the lab experiment, we collected and analyzed one discrete sample at 10 time points over five days.

In the field experiment, water was pumped from Waquoit Bay, MA, using a submersible well pump. The water passed through the canister filters and then into a bucket to overflow, as shown in Figure 2-1. After the canister filters and before the bucket, a sampling valve was installed and used to collect the discrete samples. For this experiment, we collected and analyzed one discrete sample at eight time points over eight days.

The discrete samples were collected in copper tubes, sealed with a cold pressure welder and extracted in the lab (Jenkins et al., 2010). Noble gas abundances were measured on a pulse counting quadrupole mass spectrometer (Stanley et al., 2009a). This method deter-

mines the concentration of each gas (in $\mathrm{cm}_{S T P}^{3} \mathrm{~g}^{-1}$ or $\mathrm{mol} \mathrm{kg}^{-1}$ ), with a combined standard uncertainty of $0.2-0.3 \%$ for each gas. In order to compare the GEMS data to the discrete samples, we must convert the GEMS measurements of saturation anomalies to mole ratios. Therefore, the choice of solubility function directly influences our estimates of the accuracy of the GEMS.

For Ne and Ar, we used the solubility determinations of Hamme and Emerson (2004a) who determined the solubilities of $\mathrm{Ne}$ and $\mathrm{Ar}$ in fresh water and seawater with an accuracy of 0.30 and $0.13 \%$ respectively, by equilibration with air. For $\mathrm{Kr}$ and Xe in fresh water (the lab experiment), we used the solubility determinations of Benson and Krause Jr (1976) who determined solubilities of all five stable noble gases in freshwater (but not salt water) with a stated accuracy of 0.1-0.2 \% . For the field experiment (sampling seawater), we used the solubility of Weiss and Kyser (1978) and the solubility of Wood and Caputi (1966) for Xe, fit by Hamme following the procedure in Hamme and Emerson (2004a), who determined the solubilities in both fresh water and seawater. Recent works have drawn the seawater solubilities of $\mathrm{Kr}$ and Xe into question because they are not consistent with oceanic data 
and have not been verified by multiple investigators (Hamme and Severinghaus, 2007). Thus the Kr and Xe solubilities of Weiss and Kyser (1978) and Wood and Caputi (1966) have uncertainties of 1-2 \% (Hamme and Severinghaus, 2007; Stanley et al., 2009b), which results in increased uncertainty in our accuracy estimates during the field experiment for all mole ratios except $\mathrm{Ne} / \mathrm{Ar}$.

We define the accuracy as the average magnitude (absolute value) of the relative percent difference between the GEMS and discrete samples, with both data sets expressed in terms of gas mole ratios. We filtered the GEMS data with a 7-min running mean filter and then calculated the average mole ratios over a 7 -min period centered around the time each discrete sample was collected. The choice of averaging time (from 3-15 min) did not significantly affect the estimated accuracy. The mole ratios obtained by the GEMS are determined from the measured saturation anomaly and the gas solubility at the in situ salinity and temperature.

In the lab experiment, the relative accuracy of the GEMS was $0.9 \%$ or better for all gas mole ratios (Figure 2-3). The experimentally-determined accuracy was 0.8, 0.4, 0.9, $0.8,0.8$, and $0.6 \%$ for the mole ratios of $\mathrm{Ne} / \mathrm{Xe}, \mathrm{Ne} / \mathrm{Kr}, \mathrm{Ne} / \mathrm{Ar}, \mathrm{Ar} / \mathrm{Xe}, \mathrm{Ar} / \mathrm{Kr}$, and $\mathrm{Kr} / \mathrm{Xe}$ respectively. The relative percent accuracy is the same for $\mathrm{Ne} / \mathrm{Xe}$ as for $\mathrm{Xe} / \mathrm{Ne}$, and likewise for the other gas mole ratios.

In the field experiment, the accuracy of the GEMS was $0.6,0.7$, and $0.4 \%$ for $\mathrm{Ne} / \mathrm{Kr}$, $\mathrm{Ne} / \mathrm{Ar}$, and $\mathrm{Ar} / \mathrm{Kr}$ (Figure A-3). The accuracy of the ratios with Xe was substantially worse: 2.5, 2.0, and $2.4 \%$ for $\mathrm{Ne} / \mathrm{Xe}, \mathrm{Ar} / \mathrm{Xe}$, and $\mathrm{Kr} / \mathrm{Xe}$, respectively. However, we found that we could improve the accuracy for the ratios with Xe by using the discrete samples to calibrate the GEMS (Figures A-2-A-4). We plotted the measured ratio, normalized to equilibrium for the GEMS versus the discrete samples and calculated a linear fit. The slope, $m$, and intercept, $b$ were used to calibrate the GEMS data.

$$
\left[\frac{\left(\frac{n_{N e}}{n_{X e}}\right)_{w}}{\left(\frac{n_{N e}}{n_{X e}}\right)_{e q}}\right]_{\text {discrete }}=m\left[\frac{\left(\frac{n_{N e}}{n_{X e}}\right)_{w}}{\left(\frac{n_{N e}}{n_{X e}}\right)_{e q}}\right]_{G E M S}+b
$$

The $\mathrm{R}^{2}$ values for the fit were $0.93,0.85$, and 0.73 for $\mathrm{Ne} / \mathrm{Xe}, \mathrm{Ar} / \mathrm{Xe}$, and $\mathrm{Kr} / \mathrm{Xe}$, respectively (Figure A-2). Using this technique to adjust the GEMS data, the accuracy became 0.6, 0.4, and $0.4 \%$ for $\mathrm{Ne} / \mathrm{Xe}, \mathrm{Ar} / \mathrm{Xe}$, and $\mathrm{Kr} / \mathrm{Xe}$, respectively. Similarly, with calibration, the accuracy of the lab measurements also improved somewhat, to 0.6, 0.4 and $0.4 \%$ for $\mathrm{Ne} / \mathrm{Xe}, \mathrm{Ar} / \mathrm{Xe}$, and $\mathrm{Kr} / \mathrm{Xe}$, respectively. Some of the error observed in the field may be associated with errors in the solubility of $\mathrm{Kr}$ and Xe. However, since the offset between the GEMS and discrete samples is not constant and seems to vary as a function of the magnitude of disequilibrium, not all of it can be explained by solubility errors.

We conclude that the GEMS can reliably obtain accuracy of $0.9 \%$ or better for $\mathrm{Ne} / \mathrm{Kr}$, 
$\mathrm{Ne} / \mathrm{Ar}$, and $\mathrm{Ar} / \mathrm{Kr}$ using air as the only calibration standard. For Xe, if accuracy of $0.9 \%$ or better is desired, obtaining some discrete samples for calibration purposes is recommended.

We believe the reduced accuracy for Xe in the field experiment may be related to matrix effects (Stanley et al., 2009a). Variability in the total pressure and/or the pressure of specific molecules may cause non-linearities in the relationship between gas pressure and signal intensity at the detector (e.g., due to altering the ionization efficiency for the gas of interest). Xe is likely to be the most sensitive to these matrix effects because it is the least abundant gas we measure (closest to the detection limit), and since its saturation state is the most variable (Stanley et al., 2009b; Hamme and Severinghaus, 2007). In unpurified air, the mole fractions of $\mathrm{O}_{2}$ and $\mathrm{N}_{2}$ are $\sim 10^{9}$ times greater than Xe. Therefore, even though the getters remove $>98 \%$ of the active (non-noble) gas content, the pressure of $\mathrm{N}_{2}$ and $\mathrm{O}_{2}$ is still far greater than the pressure of Xe after purification. Furthermore, in the field, biogenic gases such as $\mathrm{O}_{2}$ and $\mathrm{CO}_{2}$ will likely be more variable in abundance, and farther from equilibrium, compared to the lab experiment performed with distilled water. Therefore, we expect greater differences between the headspace and air composition in the field, leading to larger matrix effects.

Additionally, even if we could remove $100 \%$ of the active gas, the pressure of ${ }^{40} \mathrm{Ar}$ would still be 300000 times greater than the pressure of ${ }^{129} \mathrm{Xe}$, and therefore the measured pressure of Xe may be affected by changes in the pressure of Ar (Stanley et al., 2009a). A matrix effect caused by other noble gases may be more apparent at larger deviations from equilibrium. In our field data set, the noble gas mole ratios were on average farther from equilibrium, and also had larger maximum magnitudes compared to the lab data set. For example, the largest saturation anomalies measured for $\mathrm{Ne} / \mathrm{Xe}, \mathrm{Ar} / \mathrm{Xe}$, and $\mathrm{Kr} / \mathrm{Xe}$ were 6.4, 3.9 , and $3.1 \%$ in the field and 4.3, 1.7, and $2.1 \%$ in the lab, respectively, based on the discrete samples.

Notably, precision and accuracy are also degraded when the instrument experiences vibrations, such as on a ship (see Supporting Information).

Since we only analyzed one sample at each time point, we cannot determine whether any of the discrete samples may be inaccurate due to sampling or measurement problems; however, by using samples at 8-10 time points, we believe we have a good estimate of the overall accuracy. Comparing the two methods has an additional source of error: the discrete samples capture the instantaneous gas composition at the time the tube was sealed, whereas the GEMS averages over several minutes, with the e-folding time varying for each gas. The GEMS achieves similar accuracy to other methods that are much more expensive and labor-intensive. 

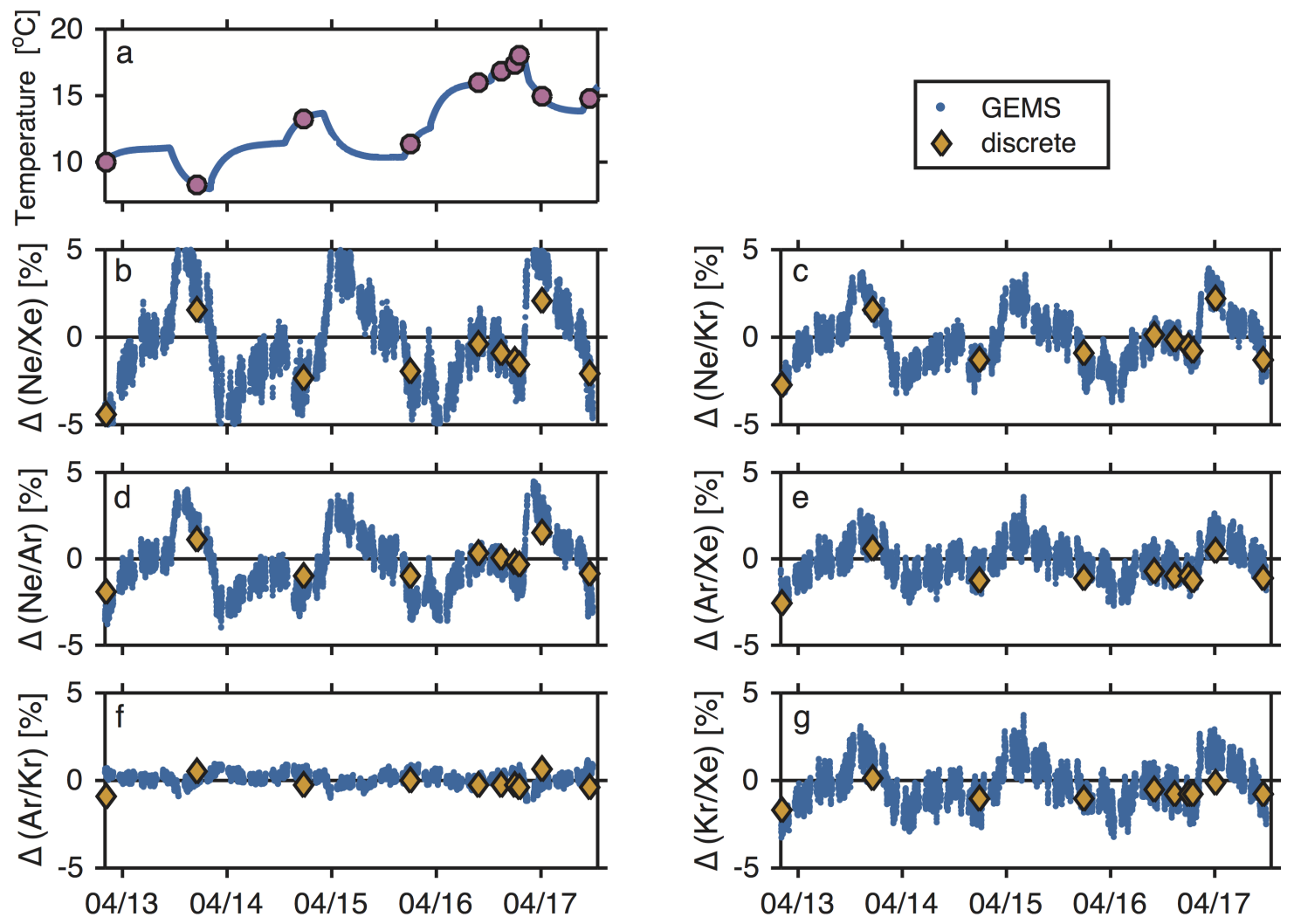

Figure 2-3: Results of a lab experiment to determine the accuracy of the GEMS. a) In situ temperature throughout the experiment (blue line) and the time each sample was collected (pink circles). b-g) Individual measurements by the GEMS filtered with a 7-min running mean filter (blue dots). Discrete samples (yellow diamonds), with the measured concentration converted to saturation anomalies. The height of the diamonds is equal to the measurement uncertainty. 


\subsubsection{Equilibration timescale}

When sampling the headspace, the signal intensity for each selected ion reflects a weighted average of the concentration over the equilibration timescale of the system. To determine the equilibration timescale, we switched between sampling water of two different gas compositions: air-equilibrated water and freshly distilled water. We fit the instrument response to a kinetic equation (Cassar et al., 2009). The signal intensity or concentration, C, for each noble gas can be modeled as

$$
C_{t}=\left[C_{i}-C_{f}\right] \exp (-t / \tau)
$$

where $C_{i}$ is the initial signal intensity (before switching the water composition), $C_{f}$ is the final intensity (after stabilization), $C_{t}$ is the intensity at any time $t$, and $\tau$ is the e-folding time of the instrument. Specifically, $\tau=\mathrm{t}_{1 / 2} / \ln (2)$, with $t_{1 / 2}$ the time at which the signal intensity is halfway between $C_{f}$ and $C_{t}$. By rearranging equation 2.8, we can plot the data as a linear equation of the form $y=m x$ where $x=t, m=\tau^{-1}$, and

$$
y=\ln \left(\frac{C_{t}-C_{f}}{C_{i}-C_{f}}\right) .
$$

For water at $20{ }^{\circ} \mathrm{C}$ and a water flow rate of $18 \mathrm{~cm}^{3} \mathrm{~s}^{-1}$, the e-folding times were found to be 410(54) s for Ne, 240(80) s for Ar, 190(80) s for Kr, and 90(10) s for Xe, where the numbers in parentheses are the standard uncertainty (Figure 2-4). These estimates are based on at least three measurements of the e-folding time for each gas; each measurement took $\sim 2 \mathrm{~h}$. The e-folding time increases with decreasing solubility. A greater proportion of the lower solubility gas must transfer between the water and the headspace in order for the two phases to re-equilibrate, causing the equilibration time to increase. Other investigators have noticed that lower solubility gases equilibrate less efficiently across Liqui-Cel membrane contactors (Cassar et al., 2009).

\subsubsection{Pilot field study}

To demonstrate the utility of the GEMS, we conducted a pilot field study in Waquoit Bay, MA, USA. We installed the mass spectrometer and laptop in an unheated boathouse, and we installed the equilibration components just outside the boathouse. The filter and bucket were placed on a bench, and the remainder of the equilibration equipment was installed inside a wooden box to shelter it from precipitation. A hole in the the wall of the boathouse was used to connect the capillary between the multiposition valve and the mass spectrometer.

To sample water, we deployed a submersible well pump $\sim 60 \mathrm{~m}$ offshore, in an average water depth of $1 \mathrm{~m}$. The water pump and two temperature/salinity/depth sensors (RBR Concerto) were attached to a hollow PVC pipe mounted on a cement block. The 

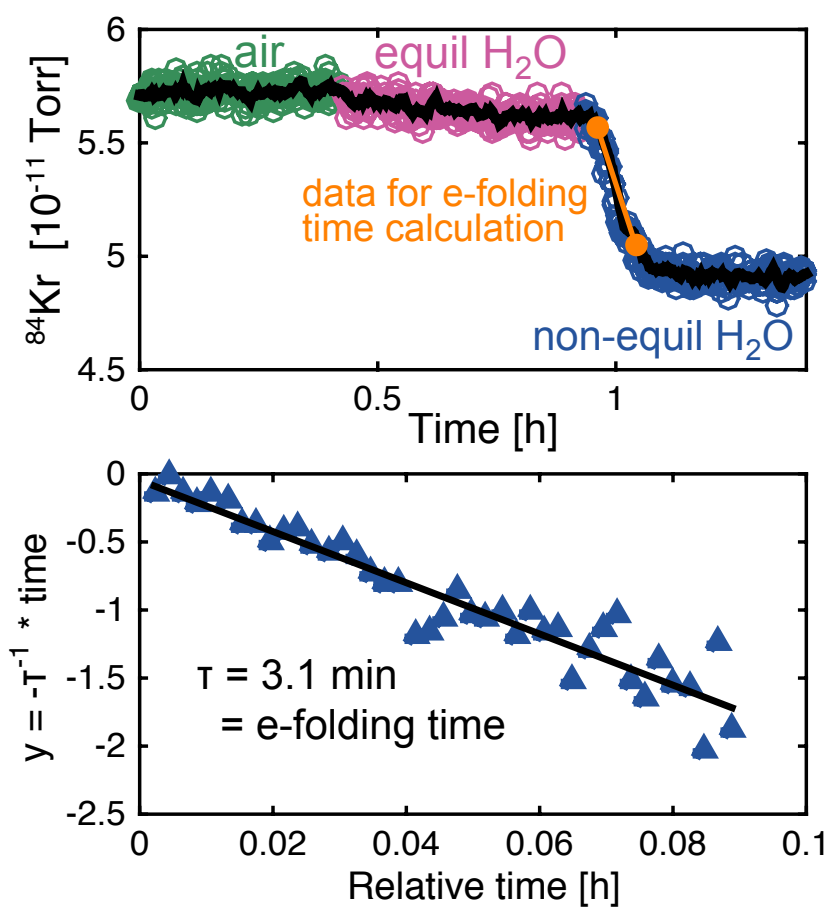

Figure 2-4: Results of an experiment to determine the equilibration timescale for each gas. a) ${ }^{84} \mathrm{Kr}$ signal intensity during an experiment switching from air, to equilibrated water, to non-equilibrated water. The orange circles and line show the portion of the data that is used to calculate the e-folding time. b) Calculation of the e-folding time from a linear regression of $y=-\tau^{-1} t$, with $\tau$ the e-folding time and $t$ the time. 
water pump was installed with the intake at an average depth of $0.4 \mathrm{~m}$, and the temperature/salinity/depth sensors were installed at an average depth of 0.4 and $0.8 \mathrm{~m}$. An additional temperature sensor $\sim 1 \mathrm{~m}$ above the water level was attached to the PVC pipe, to monitor air temperature. Wind speed data was taken from the Waquoit Bay Carriage House weather station, $200 \mathrm{~m}$ north (inshore) of the water pump (NNERRS, 2016).

The GEMS collected data for one month. In Figure 2-5, we show the data from December 16-24, the time period when discrete samples were collected for method validation purposes. We typically checked on the system twice per day (morning and early evening), and it otherwise operated unattended. During these checks we replaced the filter socks (roughly once per day), replaced the canister filters (once per week), and replaced the desiccant and membrane contactor (once every five days). We also plotted the mass spectrometer data, flow rates, and temperatures, to verify that the system was operating as intended. We obtained a near-continuous time-series with occasional gaps resulting from the submersible pump coming out of the water at the lowest tides (e.g., midnight on Dec 19 and afternoon on Dec 21 in Figure 2-5). This type of study (sub-hourly measurement frequency, over a month) would not be practical with traditional sampling and analysis methods (Machler et al., 2012).

In Figure 2-5, the GEMS data for $\Delta(\mathrm{Ne} / \mathrm{Xe})$ was calibrated using the discrete samples, and the other data is unadjusted. The error bars for $\Delta(\mathrm{Ne} / \mathrm{Xe})$ and $\Delta(\mathrm{Ar} / \mathrm{Kr})$ are larger in the field compared to the laboratory-based experiment, due to the larger uncertainties in the seawater solubility of $\mathrm{Kr}$ and Xe compared to the fresh water solubility. See the Accuracy section for more details.

The precision, accuracy, and response time of the system were sufficient to resolve substantial variability in noble gas saturation anomalies throughout the time-series. This variability was associated with changes in wind speed, water temperature, and air temperature (Figure 2-5). To determine whether the observations were consistent with our scientific understanding of physical controls on gas saturation state, we used a simple model. The model was forced with wind speed, temperature, and salinity observations, and the gas exchange parameterization of Nicholson et al. (2011), which includes separate terms for diffusive and bubble-mediated gas exchange (Manning and Nicholson, 2016). We assumed a fixed $1 \mathrm{~m}$ water depth. We initialized the model on Dec 16, 12:30 pm using the measured saturation anomalies of the first discrete sample. This model is an oversimplification because it does not account for the movement of water masses (e.g., due to tides) and the variable water depth. However, the model helps us to determine how much of the variability can be explained by air-sea gas exchange and changes in temperature/salinity.

The model predicted many similar features to the observations. For example, the model and observations show similar amplitude in the saturation anomalies, with $\mathrm{Ne} / \mathrm{Xe}$ having the widest range in saturation anomalies and $\mathrm{Ar} / \mathrm{Kr}$ the least. Additionally, the timing 

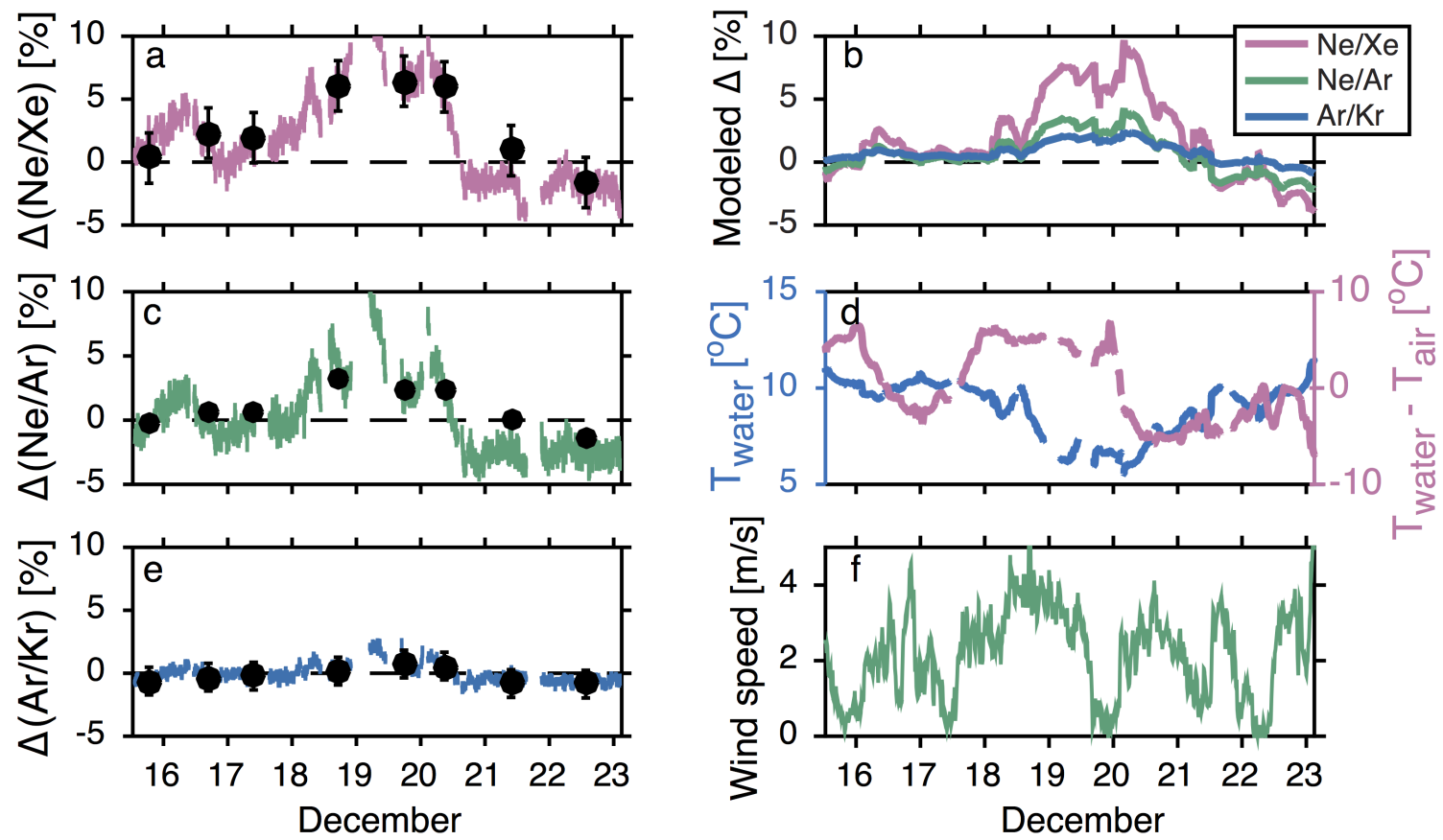

Figure 2-5: GEMS and discrete measurements of a) $\Delta(\mathrm{Ne} / \mathrm{Xe})$, c) $\Delta(\mathrm{Ne} / \mathrm{Ar})$, and e) $\Delta(\mathrm{Ar} / \mathrm{Kr})$ in Waquoit Bay, MA. The Ne/Xe data has been calibrated using discrete samples, as described in the Accuracy section. b) Modeled gas distributions forced by the measured temperature, salinity, and wind speed. d) Variability in water temperature (blue line) changes the measured saturation anomaly, and the air-water temperature difference (pink line) affects the air-sea gas flux. e) Wind speed also affects the gas flux. Date ticks represent midnight local time. 
of changes is similar in the model and data. For example, the model and data show the saturation anomalies increasing from near-equilibrium to positive values on December 1820, and decreasing from positive values to negative values on December 20-21. The changes in saturation anomalies are linked to changes in the water temperature and the air-water temperature difference. High resolution data such as this could be used to examine other processes such as tidally driven flows/mixing (e.g., by combining the GEMS with current velocity measurements) and to infer the rates of biological processes (e.g., by combining the GEMS with $\mathrm{O}_{2}$ measurements). In the Supporting Information, we describe in detail several potential applications of the GEMS, including lab-based tank experiments, introduced tracer studies, and parameterizing physical versus biological gas fluxes.

\subsubsection{Comparison with other published methods}

The GEMS dramatically increases throughput, decreases labor, and decreases costs compared to traditional discrete sampling and analysis methods. The GEMS has improved accuracy and time resolution compared to another portable method that measures Ar and Kr, but not Ne or Xe (Machler et al., 2012). The accuracy is similar to (Brennwald et al., 2013a; Sano and Takahata, 2005; Beyerle et al., 2000) or somewhat less accurate than (Stanley et al., 2009a; Hamme and Severinghaus, 2007) laboratory-based methods that cause much higher cost and labor. Additionally, the laboratory-based methods with higher precision require much more expensive instrumentation (over 250000 USD), have higher analysis costs ( 500 USD per sample), and have lower sample throughput ( $\sim 4$ samples per day), since each sample takes several hours to extract and analyze. In contrast, our system is less expensive to build ( $\sim 50000$ USD in 2013), requires minimal consumables, and can collect an endless number of samples with a time resolution of 90-410 s. Thus, the GEMS enables continuous, real-time measurements of four noble gases, with a sampling frequency (sub-hourly) that would be challenging to achieve via traditional methods (Machler et al., 2012).

The main disadvantage is that the described GEMS system does not determine the gas concentrations; instead it determines their mole ratios. In the future, we plan to test modifications to the GEMS that will enable the determination of the gas concentrations, in addition to their mole ratios. For example, the GEMS could be used along with a second mass spectrometer measuring $\mathrm{O}_{2} / \mathrm{Ar}$ ratios (e.g., an equilibrator inlet mass spectrometer (Cassar et al., 2009), a membrane inlet mass spectrometer (Tortell, 2005; Virkki et al., 1995; Kana et al., 1994), or the GEMS system described above, with the getter chambers eliminated) and a well-calibrated sensor for $\mathrm{O}_{2}$ concentration. The $\mathrm{O}_{2} / \mathrm{Ar}$ ratio and the $\mathrm{O}_{2}$ concentration could be used to derive the Ar concentration (Eveleth et al., 2014; Hamme et al., 2012) and the other noble gas concentrations could be determined from the GEMS noble gas ratios and the Ar concentration. Another potential modification is changing the system to measure individual samples, instead of a continuous gas stream (Visser et al., 
2013; Machler et al., 2012).

\subsection{Conclusions}

We have described the gas equilibration mass spectrometer (GEMS), a new field-deployable method for continuous measurement of the mole ratios of four noble gases (Ne, Ar, Kr, and $\mathrm{Xe}$ ) dissolved in water. In the lab, the precision is $0.7 \%$ or better, and in a variabletemperature environment the precision is $1.0 \%$ or better. The accuracy is $0.9 \%$ or better for all gas ratios in the lab. In the field (and/or at greater disequilibrium) the accuracy is $0.7 \%$ or better for $\mathrm{Ne} / \mathrm{Kr}, \mathrm{Ne} / \mathrm{Ar}$, and $\mathrm{Ar} / \mathrm{Kr}$ and $2.5 \%$ or better for $\mathrm{Ne} / \mathrm{Xe}, \mathrm{Ar} / \mathrm{Xe}$, and $\mathrm{Kr} / \mathrm{Xe}$, but can be improved through calibration with discrete samples to $0.6 \%$ or better.

\subsection{Acknowledgments}

The authors thank Kevin Cahill, Josh Curtice, James Rassman, and Zoë Sandwith for assistance with lab and field work, Bill Jenkins for processing the noble gas data from discrete samples, and David Nicholson for discussions about the field data. The manuscript was improved thanks to the thoughtful suggestions of two anonymous reviewers. Funding was provided by NSF award OCE-1060840 and a WHOI Innovative Technology award to RHRS, and scholarships from NSERC and CMOS to CCM.

\subsection{Supplemental information}

The supplemental information for this chapter includes lists of suppliers and parts numbers, photos of the system, a diagram of the getter chambers, descriptions of some alternative configurations of the system, and further details on the calibration of field data. It can be found in Appendix A. 


\section{Chapter 3}

\section{Quantifying air-sea gas exchange using noble gases in a coastal upwelling zone}

This chapter was published in IOP Conference Series: Earth and Environmental Science as part of the refereed Proceedings of the 7th International Conference on Gas Transfer at Water Surfaces and is reprinted here under license from IOP Publishing Ltd. Content from this work may be used under the terms of the Creative Commons Attribution 3.0 licence. Some of the software used in this chapter was published on Zenodo and GitHub and as a supplement to the paper. Data is available on Zenodo and as a supplement to the published paper. Any further distribution of this work must maintain attribution to the authors and the title of the work, citation and DOI.

CC Manning, RHR Stanley, DP Nicholson, and ME Squibb (2016) Quantifying air-sea gas exchange using noble gases in a coastal upwelling zone. IOP Conference Series: Earth and Environmental Science 35, 012017 doi: 10.1088/1755-1315/35/1/012017

CC Manning and DP Nicholson (2016) gas_toolbox: Revised MATLAB code used in Manning et al. GTWS-7 proceedings. Zenodo. doi: 10.5281/zenodo.46569. The latest version of the functions is available at http://github.com/dnicholson/gas_toolbox.

CC Manning, RHR Stanley, and DP Nicholson (2016) Dataset from "Quantifying air-sea gas exchange using noble gases in a coastal upwelling zone." Zenodo. doi: 10.5281/zenodo.56695.

The supplemental information for this chapter can be found in Appendix B. 


\subsection{Abstract}

The diffusive and bubble-mediated components of air-sea gas exchange can be separately quantified using time-series measurements of a suite of dissolved inert gases. We have evaluated the performance of four published air-sea gas exchange parameterizations using a five-day time-series of dissolved $\mathrm{He}, \mathrm{Ne}, \mathrm{Ar}, \mathrm{Kr}$, and Xe concentration in Monterey Bay, CA. We constructed a vertical model including surface air-sea gas exchange and vertical diffusion. Diffusivity was measured throughout the cruise from profiles of turbulent microstructure. We corrected the mixed layer gas concentrations for an upwelling event that occurred partway through the cruise. All tested parameterizations gave similar results for Ar, $\mathrm{Kr}$, and Xe; their air-sea fluxes were dominated by diffusive gas exchange during our study. For He and $\mathrm{Ne}$, which are less soluble, and therefore more sensitive to differences in the treatment of bubble-mediated exchange, the parameterizations gave widely different results with respect to the net gas exchange flux and the bubble flux. This study demonstrates the value of using a suite of inert gases, especially the lower solubility ones, to parameterize air-sea gas exchange.

\subsection{Introduction}

Noble gases dissolved in seawater are biologically and chemically inert, making them excellent tracers of numerous physical processes that control gas saturation states in the ocean (e.g., bubble mediated and diffusive gas exchange, temperature change, atmospheric pressure change, ice melting, diapycnal mixing, deepwater formation and ventilation) (Loose and Jenkins, 2014; Nicholson et al., 2011; Stanley et al., 2009b). By simultaneously measuring several inert gases with a range of physical properties (e.g., diffusivity, solubility, and dependence of solubility on temperature), we can separately quantify many of these physical processes (Hamme and Severinghaus, 2007; Hamme and Emerson, 2002). For example, the solubility of the noble gases decreases with decreasing atomic mass, and the lower solubility noble gases ( $\mathrm{He}$ and $\mathrm{Ne}$ ) have a larger portion of their total gas exchange flux driven by bubbles. The dependence of bubble flux on solubility can be explained as follows: for lower solubility gases the atmospheric concentration is high relative to the water concentration, and thus when air bubbles dissolve in the water, the bubbles will generate a larger percent increase in the gas concentration, compared to a higher solubility gas. Additionally, the saturation state of the higher solubility (heavier) noble gases changes more dramatically in response to surface heating/cooling, and mixing of water masses with different temperatures, due to the stronger dependence of the solubility on temperature compared to the lower solubility (lighter) noble gases. Parameterizations of physical processes from inert gases can be applied to bioactive gases, to obtain more accurate estimates of processes including bio- 
logical productivity (Hamme and Emerson, 2006; Stanley et al., 2009b) and denitrification (DeVries et al., 2012; Chang et al., 2012).

Here, we use a quasi-Lagrangian time-series of the five stable noble gases ( $\mathrm{He}, \mathrm{Ne}, \mathrm{Ar}, \mathrm{Kr}$, and $\mathrm{Xe}$ ) in Monterey Bay, CA, along with a vertical model, to evaluate the performance of four gas exchange parameterizations, which differ in their treatment of diffusive and bubblemediated air-sea gas exchange. These data are some of the few published measurements of a suite of noble gases in a coastal upwelling zone, where environmental factors such as increased levels of surfactants and reduced fetch for wind wave generation may cause the relationship between wind speed and gas exchange rates to differ from the relationship in the open ocean (Upstill-Goddard, 2006; Frew, 1997). In a forthcoming publication, we will apply the noble gas-based parameterizations of physical processes to $\mathrm{O}_{2}$ concentration and isotope measurements, in order to quantify net community production and gross primary production during the time-series.

\subsection{Methods}

\subsubsection{Cruise description}

We participated in a seven-day quasi-Lagrangian cruise in Monterey Bay, CA, USA, on the R/V Western Flyer, Sept 27-Oct 3, 2014. During the cruise, rosette casts to $\sim 180 \mathrm{~m}$ were conducted four times per day, at roughly 06:00, 12:00, 18:00, and 00:00 local time. Immediately prior to nearly every cast, vertical profiles of microscale turbulence to $\sim 70$ m were obtained using a vertical microstructure profiler (VMP-200, Rockland Scientific). Rates of turbulent kinetic energy dissipation were calculated using two perpendicular airfoil type shear probes on the VMP-200. The Nasmyth spectrum was used to recursively estimate dissipation and diapycnal diffusivity, $K_{z}$ (Wolk et al., 2002). Roughly three high quality turbulence profiles were obtained every $6 \mathrm{hr}$.

All CTD casts were performed and all noble gas samples were collected in the vicinity of mooring M1 (21 km west of Moss Landing, CA), which is maintained by the Monterey Bay Aquarium Research Institute (Figure 3-1). Starting on Sept 28, 12:35 and throughout the rest of the cruise, we selected where to collect CTD and turbulence profiles by following an autonomous underwater vehicle (AUV) drifting at $\sigma_{t} \approx 25.2 \mathrm{~kg} \mathrm{~m}^{-3}$ (25-45 m depth), near the base of the thermocline. Thus, the cruise was quasi-Lagrangian, but with respect to the base of the thermocline, rather than the mixed layer. We also collected noble gas samples from one offshore cast (37 km west of Moss Landing, CA), which indicated that there is minimal spatial variability in noble gas distributions, with respect to both saturation anomaly and concentration. 


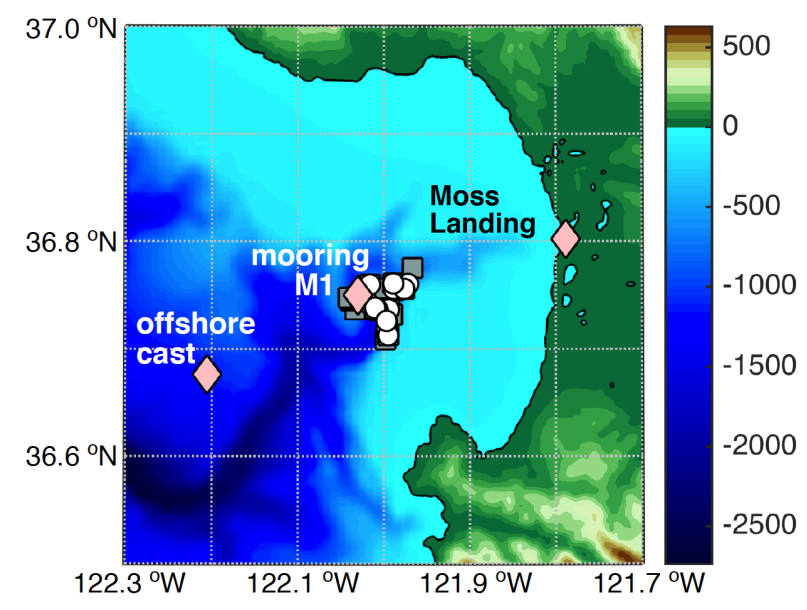

Figure 3-1: Map of the study site. Pink diamonds indicate the locations of Moss Landing, CA, mooring M1, and the offshore cast. Grey squares are locations of CTD casts and white circles are locations that surface samples were collected (sometimes colocated with CTD casts, sometimes in between casts). Colorbar shows elevation and bathymetry in meters.

\subsubsection{Noble gas data}

In this paper, we present noble gas data ( $\mathrm{He}, \mathrm{Ne}, \mathrm{Ar}, \mathrm{Kr}$, and Xe concentrations) from discrete samples, which were collected from the ship's underway seawater line and from Niskin bottles. The samples were collected in copper tubes, sealed with a cold pressure welder, and extracted in the Isotope Geochemistry Facility at Woods Hole Oceanographic Institution (Jenkins et al., 2010). Noble gas abundances were measured on a pulse counting quadrupole mass spectrometer using the system described in Stanley et al. (2009a). The dissolved concentration of each gas (in $\mathrm{cm}_{S T P}^{3} \mathrm{~g}^{-1}$ or $\mathrm{mol} \mathrm{kg}^{-1}$ ) was determined, using selected ion monitoring (Stanley et al., 2009a) and including isotope dilution analysis for $\mathrm{Kr}$ and Xe. The estimated error (combined precision and accuracy) is $\pm 0.27,0.27,0.24$, 0.25 , and $0.27 \% \mathrm{RSD}$ (relative standard deviation) for $\mathrm{He}, \mathrm{Ne}, \mathrm{Ar}, \mathrm{Kr}$, and Xe respectively. As we did not analyze samples in replicate from this dataset, the errors are based on a different dataset of duplicate samples that were collected and analyzed by the same methods immediately following this dataset. Three samples with evidence of air contamination (based on anomalies in the relative excess concentration of the five noble gases in the sample, compared to the other samples at a similar depth) were eliminated from the dataset.

Since the rate of gas exchange is dependent on the deviation of the gas concentration from equilibrium, accurate solubility functions are critical for accurate parameterization of air-sea gas exchange and other physical processes. Hamme and Emerson (2004) have published high-quality solubility data for $\mathrm{Ne}$ and $\mathrm{Ar}$ (errors of \pm 0.30 and $0.13 \%$ respectively) based on equilibration of water with air at a range of temperatures and salinities (Hamme and Emerson, 2004a). For the other gases in seawater, researchers have typically used the solubility data of Weiss (1971) for He, Weiss and Kyser (1978) for Kr, and Wood and Caputi 
(1966) for Xe. Errors of $\sim 2 \%$ may be present in these solubility functions because they were determined for $1 \mathrm{~atm}$ of each pure gas, and these data must be extrapolated over several orders of magnitude to calculate the solubility with respect to air. For example, Weiss (1971) determined the solubility of $\mathrm{Ne}$ in addition to He, and his reported seawater Ne solubility was $\sim 1.5 \%$ Hamme and Emerson (2004a). Additionally, the published Kr and Xe solubilities in seawater are subject to uncertainties in the atmospheric mole fraction of each gas (Aoki and Makide, 2005). Therefore, for He, Kr, and Xe, we use currently unpublished solubility data from Lott and Jenkins (personal communication, 2015), who determined the solubility of $\mathrm{He}, \mathrm{Kr}$, and Xe from 0 to $36.6 \mathrm{PSS}$, and from $<1$ to $35{ }^{\circ} \mathrm{C}$ by equilibration of water with air. An additional advantage of the Lott and Jenkins dataset is that the solubilities were all measured on the same samples, using the same instrument. Thus the He, Kr, and Xe solubilities of Lott and Jenkins should be more internally consistent compared to data compiled from three different papers (two different laboratories). At the typical sea surface conditions during our study ( $\mathrm{S}=34.4 \mathrm{PSS}, \mathrm{T}=16{ }^{\circ} \mathrm{C}$ ), the solubilities of Lott and Jenkins are $2.2 \%$ higher for $\mathrm{He}, 1.3 \%$ higher for $\mathrm{Kr}$, and $0.1 \%$ less for Xe, compared to the published solubilities.

In the main paper, we show the data and model results calculated with the Hamme and Emerson (2004) solubilities for $\mathrm{Ne}$ and Ar, and the Lott and Jenkins solubilities for He, $\mathrm{Kr}$, and Xe. In the Supplemental Information Information, we show the results using the published solubilities for $\mathrm{He}, \mathrm{Kr}$, and $\mathrm{Xe}$, and we provide the noble gas concentration data along with ancillary data (salinity, temperature, atmospheric pressure, etc.) so that it can be used by future investigators with the most accurate solubility functions available. The choice of solubility functions does not affect our overall conclusions, however, all models simulate the He data better when the Lott and Jenkins solubility is used instead of Weiss (1971).

For the gas molecular diffusivity, which also enters into the gas exchange parameterizations, we used the freshwater data of Jähne et al. (1987a) adjusted by $-0.138 \%$ per ppt salinity (-4.75\% adjustment for 34.4 PSS, the typical salinity in our study) for He, $\mathrm{Ne}, \mathrm{Kr}$, and Xe, and extrapolated values for Ar (Manning and Nicholson, 2016).

\subsection{Model description}

\subsubsection{Data input and model setup}

We developed a 1D vertical model for the time-series including diffusion and gas exchange for each noble gas. We ran the model using four different gas exchange parameterizations and evaluated the ability of each parameterization to accurately simulate the mixed layer saturation anomalies and concentrations measured throughout the cruise. The model was initialized with CTD profiles of temperature and salinity, and idealized noble gas profiles, 
described in more detail below. The model run proceeded as follows: at each time step (20 s), for each gas, the air-sea gas exchange flux was calculated and then the gas concentration in the surface box (upper $1 \mathrm{~m}$ ) was adjusted, based on this flux. Then the diffusive flux at the edge of each box (1 $\mathrm{m}$ spacing) was calculated, which mixed the change in gas concentration from gas exchange throughout the mixed layer and deeper into the water column. We used a Crank-Nicolson diffusion scheme to calculate the flux between each box, based on the vertical diffusivity rates measured every $6 \mathrm{hr}$. The vertical diffusion rate was variable with depth and with time, and the model did not explicitly prescribe a mixed layer depth. Measured diffusivity was generally orders of magnitude higher near the surface where gases are mixed much more vigorously, compared to deeper waters below the mixed layer. Our vertical diffusivity determined from microstructure profiles is more accurate compared to other published studies where the diffusivity was crudely estimated for the base of the mixed layer only.

We ran the model from Sept 28, 00:00-Oct 3, 02:00 local time. We linearly interpolated all data to the model time step of $20 \mathrm{~s}$. CTD profiles of temperature and salinity were binned to the depth grid spacing of $1 \mathrm{~m}$, from $0.5-69.5 \mathrm{~m}$, and then linearly interpolated between casts. Temperature and salinity profiles from each CTD downcast (every $6 \mathrm{hr}$ ) were used in the model for calculating the simulated gas saturation anomalies. The turbulence profiles measured prior to each CTD cast (roughly three turbulence profiles per CTD cast) were averaged, and then linearly filtered to $1 \mathrm{~m}$ depth spacing $(1-70 \mathrm{~m})$ before interpolation to the model time step.

Measurements of wind speed, atmospheric pressure, and relative humidity were obtained at 10 min intervals from sensors on mooring M1 (Figure 3-1). There were two wind sensors on the mooring: a Vaisala ultrasonic anemometer and a RM Young propeller anemometer. For this study we used data from the ultrasonic anemometer as it was specified to have higher accuracy and a lower detection limit. The wind sensors were both at $4 \mathrm{~m}$ height above the sea surface and the wind speeds were extrapolated to $u_{10}$, the wind speed at $10 \mathrm{~m}$ height, using the equation $u_{b}=u_{a}(b / a)^{0.11}$ with $a$ and $b$ the sensor and extrapolated heights respectively (Hsu et al., 1994). During the time period included in the model (excluding the upwelling event, discussed below), the root mean square deviation (RMSD) of the two sensors was $0.38 \mathrm{~m} \mathrm{~s}^{-1}(9.8 \%)$. The mean difference between the two sensors was $0.29 \mathrm{~m}$ $\mathrm{s}^{-1}$, with the anemometer giving higher wind speed $86 \%$ of the time. The model results were very similar regardless of which anemometer dataset was used. For example, the net gas exchange flux typically differed by $13 \%$ or less when the model was run with data from either anemometer.

The gas saturation anomaly was used in all the gas exchange parameterizations, and is defined in $\Delta$ notation as

$$
\Delta C=\left(C_{w} / C_{e q}-1\right) 100 \%
$$


with $C_{w}$ and $C_{e q}$ the water and saturation equilibrium gas concentrations, respectively. The equilibrium concentration of each gas at each time step was calculated with respect to local sea level pressure and humidity, specifically

$$
C_{e q}=C_{e q, r e f} \times\left(P_{s l p}-P_{H 2 O}\right) /\left(P_{r e f}-P_{H 2 O, e q}\right)
$$

where $C_{\text {eq,ref }}$ is the reference equilibrium concentration at the measured salinity and temperature for $1 \mathrm{~atm}$ total pressure of air with water vapor at $100 \%$ humidity. The pressure terms are all expressed in atm: $P_{r e f}=1 \mathrm{~atm}$ is the total reference pressure of air, $P_{s l p}$ is the local sea level pressure, and $P_{H 2 O}$ and $P_{H 2 O, e q}$ are the water vapor pressures in situ and at equilibrium (100\% relative humidity), respectively, calculated from the salinity, temperature, and relative humidity (Dickson et al., 2007).

The model was initialized with an idealized profile for each gas (Figure 3-2), since we did not have a depth profile at the start of the cruise. The concentration was fit to be similar to the other profiles at depth. The initial surface concentration of each gas was determined by fitting the model to either the concentration or saturation anomaly of the first sample, which was collected on September 28, 06:15. The fit was determined by minimizing the RMSD between the sample and model results for the four parameterizations. Fitting the models to the initial sample concentration versus saturation anomaly gave slightly different results because the saturation anomaly of each discrete sample was calculated based on the temperature and salinity of the water sampled, rather than the modeled temperature and salinity, which was interpolated from the CTD downcasts. There were sometimes differences between the real-time underway and interpolated CTD profiles (for underway samples) and offsets in the temperature and salinity profiles between the upcast and downcast (for Niskin samples). Therefore, the model and sample equilibrium concentration were sometimes different. Below, we separately report the performance of the models optimized to concentration and saturation anomaly (section 3.5).

\subsubsection{Adjustment for upwelling}

We observed evidence for upwelling of colder water with higher noble gas and lower $\mathrm{O}_{2}$ concentrations between the casts on Sept 29, 18:30 and Sept 30, 06:16 (Figure 3-3). Temperature sensors at mooring $\mathrm{M} 1$ recorded a $>1{ }^{\circ} \mathrm{C}$ decrease in temperature at 1,10 , and 20 m depth between Sept 29, 19:40 and Sept 30, 06:10. This time period coincided with the strongest winds and the strongest northerly (upwelling-favorable) winds observed during the time-series (winds were 10.0-11.8 $\mathrm{m} \mathrm{s}^{-1}$ from 18:40-21:30). Additionally, diapycnal diffusivity was enhanced during the upwelling event, from roughly 10-20 m on Sept 29, 23:30 and then from 30-40 $\mathrm{m}$ on Sept 30, 05:30, suggesting vertical propagation of wind-driven turbulence from the surface to deeper waters (Figure 3-3). 

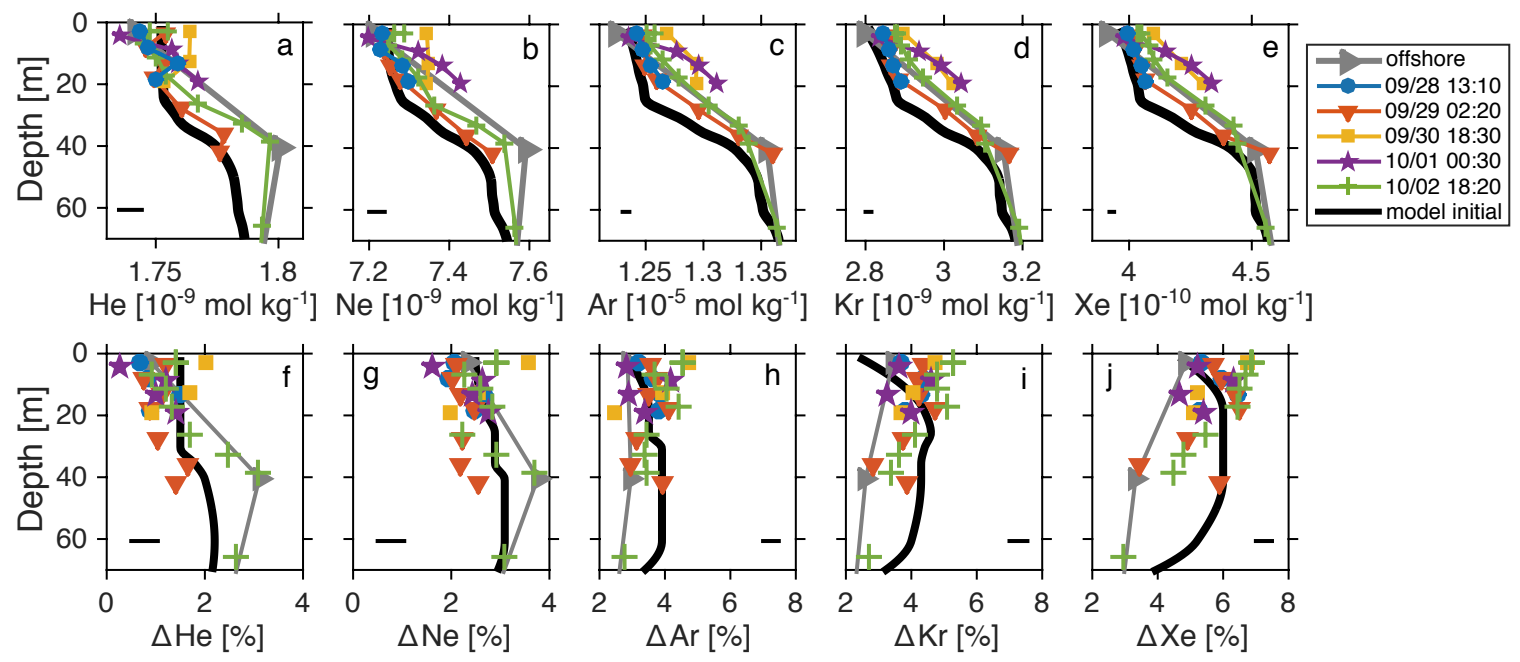

Figure 3-2: Measured profiles and idealized initial profile of noble gas concentrations (a-e) and saturation anomalies $(\mathrm{f}-\mathrm{j})$ during the cruise. The offshore cast was collected on Sept 30 , 21:30 and its location is shown in Figure $3-1$. The black line is the idealized profile that was used to initialize the model on Sept 28, 00:00. The horizontal black lines show the estimated measurement error.

Our diffusion-based model was not able to reproduce the upwelling event. Therefore, we manually reset the gas profiles at Sept 30,06:16, the time of the first cast after upwelling. The saturation anomaly of each gas throughout the upper $25 \mathrm{~m}$ was reset to the same value for all models, and these saturation anomalies were determined by minimizing the RMSD of the model results for the four different parameterizations to the first two samples collected after the upwelling event, on Sept 30, 06:33 and 07:10. Gas concentrations below $25 \mathrm{~m}$ were not adjusted because the temperature and $\mathrm{O}_{2}$ profiles below $25 \mathrm{~m}$ remained similar following the upwelling event (Figure 3-3). We fit the model to either the mean concentration or mean saturation anomaly of these two samples. This approach was identical to the approach used to determine the initial surface concentrations. We excluded samples collected during the upwelling event (at Sept 29, 19:33, and Sept 30, 01:30) when assessing the model performance.

We did not add a continuous upwelling flux throughout the cruise (e.g., using a published upwelling index for the region). Upwelling events are episodic in this region during the fall (Fitzwater et al., 2003), and our temperature and mooring data indicated that only one upwelling event occurred during our time-series, overnight Sept 29-30. Additionally, we were able to simulate the remainder of the time-series without a continuous upwelling flux.

We omitted advection from the mass balance because we did not have sufficient spatial coverage to resolve advective fluxes. This omission is likely not a serious limitation since our measurements at an offshore station suggested that the dissolved gas concentrations and saturation anomalies were very similar offshore to near M1 (Figure 3-2). Furthermore, 

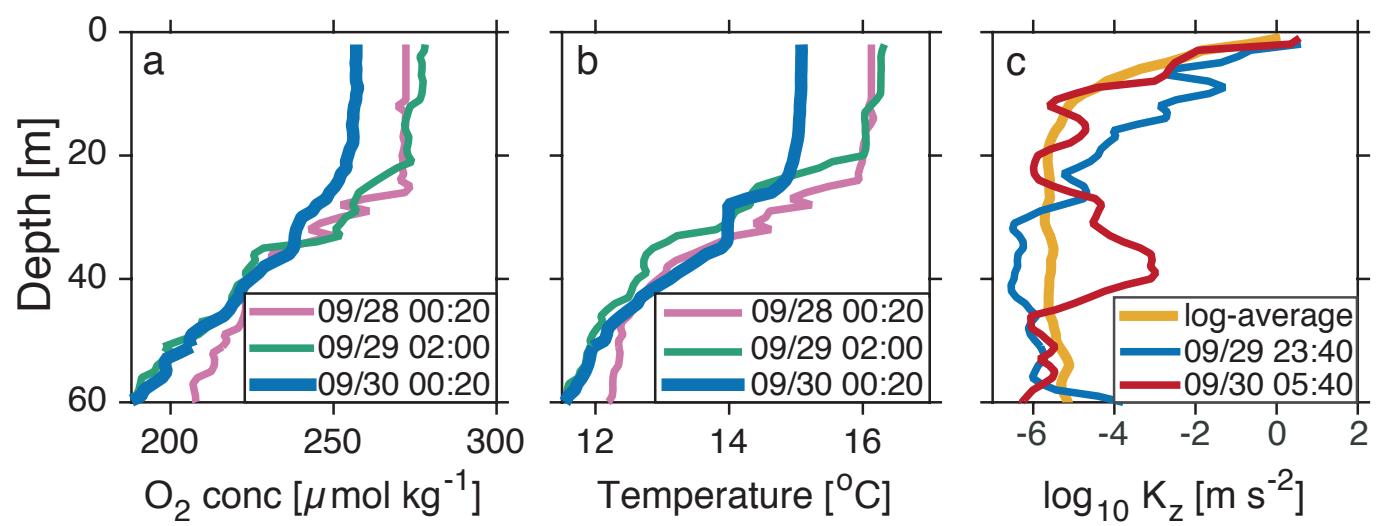

Figure 3-3: A) $\mathrm{O}_{2}$, b) temperature, and c) diapycnal diffusivity $\left(K_{z}\right)$ profiles collected during the cruise. In $\mathrm{a}-\mathrm{b}$ ), the profiles on Sept 30, 00:20, during the upwelling, showed a decrease in mixed layer temperature and $\mathrm{O}_{2}$. In c), diffusivity in the upper $40 \mathrm{~m}$ was enhanced following the upwelling, compared to the log-average (geometric mean).

we evaluate the performance of the models using only $\mathrm{He}$ and $\mathrm{Ne}$, which should be the least affected by advective fluxes and mixing between different water masses. These gases will have the lowest lateral variability in concentration and saturation anomaly, due to the very small temperature dependence of their solubilities $\left(0.2\right.$ and $0.7 \%{ }^{\circ} \mathrm{C}^{-1}$ for $\mathrm{He}$ and Ne, respectively) (Stanley and Jenkins, 2013; Nicholson et al., 2011; Hamme and Emerson, 2002). Additionally, our data show that the surface He concentration was barely altered by the upwelling event, a clearly non-Lagrangian process.

\subsubsection{Choice of gas exchange parameterizations}

We ran the model using four different gas exchange parameterizations: Liang et al. 2013 (L13), Nicholson et al. 2011 (N11), Stanley et al. 2009 (S09), and Sweeney et al. 2007 (Sw07) (Liang et al., 2013; Nicholson et al., 2011; Stanley et al., 2009b; Sweeney et al., 2007). N11 and S09 were developed from inverse models of noble gas data, with S09 using a three-year time-series of monthly profiles of $\mathrm{He}, \mathrm{Ne}, \mathrm{Ar}, \mathrm{Kr}$, and Xe concentration at the Bermuda Atlantic Time-series Study site (BATS) in tandem with a 1D oceanic mixed layer model, and N11 using a global dataset of $\mathrm{Ne}, \mathrm{Ar}, \mathrm{N}_{2} / \mathrm{Ar}$, and $\mathrm{Kr} / \mathrm{Ar}$ measurements in tandem with an ocean general circulation model. L13 used a large eddy simulation (LES) model coupled to a bubble population model to parameterize the bubble-mediated exchange and did not use oceanic gas measurements to tune the model; however, the L13 parameterization reproduced oceanic data well. With all three parameterizations, the total gas exchange flux is calculated as the sum of diffusive and bubble-mediated exchange:

$$
F_{t}=F_{d}+F_{b}
$$


with $F_{t}$ the total flux, $F_{d}$ the diffusive flux, and $F_{b}$ the bubble flux. Each parameterization further separates the bubble-mediated flux into two components:

$$
F_{b}=F_{c}+F_{p}
$$

where $F_{c}$ is the complete trapping bubble flux from typically smaller bubbles that completely dissolve and $F_{p}$ is the partial trapping bubble flux from typically larger bubbles that partially dissolve. The exact equations for $F_{d}, F_{c}$, and $F_{p}$ differ between authors, as we discuss below.

Finally, Sw07 used a global dataset of dissolved inorganic radiocarbon data to quantify the rate of uptake of anthropogenic (bomb) radiocarbon into the ocean. Sw07 does not not explicitly include the bubble-mediated component of air-sea gas exchange, i.e. it includes $F_{d}$ but not $F_{b}$. The Sw07 parameterization is within the range of other gas exchange parameterizations derived from oceanic measurements that do not include a separate term for bubble-mediated exchange (Wanninkhof, 2014; Ho et al., 2006; Naegler et al., 2006; Wanninkhof and McGillis, 1999), and is used as $F_{d}$ in N11.

All of the parameterizations use $u_{10}$ as the only environmental forcing variable. In L13, $u_{10}$ is converted to $u_{*}$ using an empirical formula for the drag coefficient (Sullivan et al., 2012; Large and Pond, 1981). Therefore, none of the models explicitly include variables such as surfactants, precipitation, and/or fetch.

S09, Sw07, and N11 all parameterize $F_{d}$ using an equation of the form

$$
F_{d}=A u_{10}^{2}(S c / 660)^{-0.5}\left(C_{e q}-C_{w}\right)
$$

Here $A$ is an empirical constant and $S c$ is the Schmidt number of the gas at the water temperature and salinity. $S c$ is defined as the ratio of the kinematic viscosity of seawater to the gas diffusivity. N11 and Sw07 both use $A=0.27 \mathrm{~cm} \mathrm{hr}^{-1}$, whereas S09 uses $A=0.30$ $\mathrm{cm} \mathrm{hr}^{-1}$, and will thus yield a diffusive flux that is $11 \%$ higher for a given wind speed and gas concentration. The comparison to $F_{d}$ calculated from L13 is more complex since $u_{*}$ is a nonlinear function of $u_{10}$. At $u_{10}=5 \mathrm{~m} \mathrm{~s}^{-1}, F_{d}$ of L13 is approximately equal to $F_{d}$ of S09 and N11. At lower wind speeds, $F_{d}$ of L13 exceeds S09 and N11 (by a factor of five at $u_{10}=1 \mathrm{~m} \mathrm{~s}^{-1}$ ), and at higher wind speeds $F_{d}$ of L13 is less than S09 and N11 (by a factor of two at $u_{10}=10 \mathrm{~m} \mathrm{~s}^{-1}$ ). The differences in $F_{d}$ for L13 (scaled to $u_{*}$ ) versus the other parameterizations (scaled to $u_{10}$ ) also have a slight dependence on the gas Schmidt number.

For the bubble-mediated fluxes $F_{c}$ and $F_{p}$, the differences between parameterizations are not simple to characterize because they are functions of additional variables such as the gas saturation anomaly, diffusivity, solubility, and atmospheric mole fraction, as well as $u_{10}$. The dependence on these factors can differ between models. For example, $F_{p}$ is scaled to the gas diffusivity, $D$, as $F_{p} \propto D^{0.5}$ in N11 and $F_{p} \propto D^{2 / 3}$ in S09. L13 sets $F_{p} \propto(S c / 660)^{-2 / 3}$. All three parameterizations assume that $F_{c}$ is independent of $D$. 
We report the differences between parameterizations for $F_{d}, F_{c}$, and $F_{p}$ in our model below. We refer the reader to the authors' original papers for more details on each parameterization (Liang et al., 2013; Nicholson et al., 2011; Stanley et al., 2009b; Sweeney et al., 2007). In the Supplemental Information, we provide MATLAB functions for calculating gas exchange fluxes using each of the four tested parameterizations, as well as several additional parameterizations that only include $F_{d}$ (Manning and Nicholson, 2016).

\subsection{Results}

We evaluated the performance of each parameterization by comparing the model results to near-surface measurements (2-4 m depth, 15 samples total). We show the gas time-series with respect to saturation anomaly as well as with respect to concentration (Figure 3-4), and we calculated the error using both parameters. For the most soluble gases, Ar, Kr, and Xe, all models gave very similar results and simulated the surface measurements well. The four models diverged for He and Ne, which have higher diffusivity and lower solubility, making them more sensitive to differences in the treatment of bubble-mediated gas exchange.
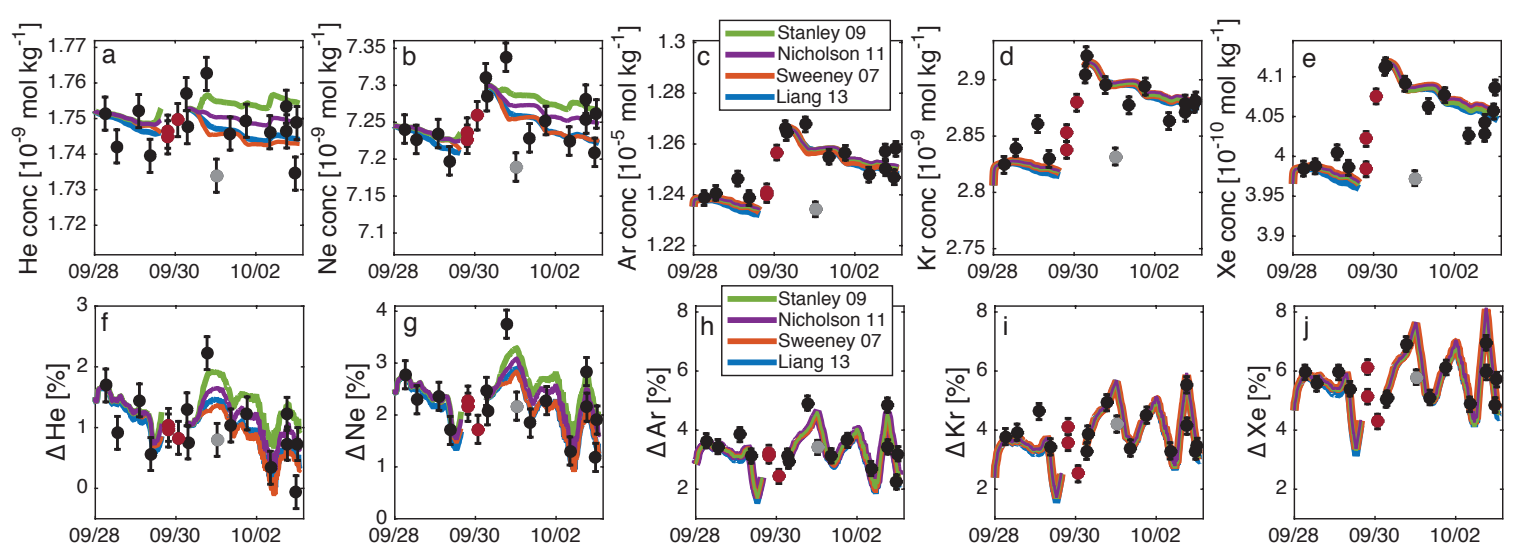

Figure 3-4: Near-surface gas concentrations (a-e) and saturation anomalies $(\mathrm{f}-\mathrm{j})$, from samples and model results. The gap in the models around midnight Sept 30 corresponds to the upwelling event. Error bars reflect one standard deviation error in concentration measurement and do not include solubility uncertainty. Maroon circles are samples during the upwelling event that were not included in the Monte Carlo error analysis. The grey circle is an unexplained outlier that was included in the error analysis.

The concentration plots for $\mathrm{Ar}, \mathrm{Kr}$, and Xe clearly show the impact of the upwelling event overnight on Sept 29-30: near-surface gas concentrations following the upwelling were 2-4\% higher than before the upwelling (Figure 3-4 c-e). The concentration increase for Ar, Kr, and Xe following the upwelling was driven by the replacement of warmer water by colder water with higher equilibrium gas concentrations (but similar saturation anomalies for each gas). In contrast, the concentrations of $\mathrm{He}$ and $\mathrm{Ne}$ changed by $<1 \%$ due to the upwelling because these gases have a lower dependence of their solubility on temperature and therefore the 
equilibrium gas concentration was similar before and after upwelling. Additionally, because the upwelling event coincided with high winds, some of the concentration increase for He and Ne during this period can be attributed to bubble-driven supersaturation rather than temperature change.

There was one sample following the upwelling, shaded grey in Figure 3-4, that fell below the model results for $\mathrm{Ar}, \mathrm{Kr}$, and Xe concentration, but not saturation anomaly. This sample was collected in warmer water $\left(16.4^{\circ} \mathrm{C}\right)$ compared to the four surface samples taken within $24 \mathrm{hr}$ of that sample $\left(15.2-15.7^{\circ} \mathrm{C}\right)$ and therefore it had a lower equilibrium gas concentration $\left(C_{e q}\right)$. It is possible that this sample may have been from a different water mass compared to the rest of the time-series, however, it was included in all of our error estimates because we had no specific reason to discount it.

For each gas, the initial surface concentration and surface concentration after upwelling were set by fitting the models to the measured concentrations of the first sample and the first two samples after upwelling, respectively. Due to the measurement uncertainty for these initialization values, we performed a Monte Carlo error analysis where the initial concentration and concentration after upwelling were simultaneously varied randomly 100 times with a normal distribution, with the optimized concentrations (Figure 3-4) set as the mean and the measurement error set as the standard deviation. We evaluated the performance of the different parameterizations by calculating the RMSD between all measured near-surface sample concentrations and the coincident modeled concentrations, for each noble gas and each parameterization. We then repeated the error analysis, but instead fit the models to the measured saturation anomaly of the first sample, and the first two samples after upwelling, and calculated the RMSD with respect to saturation anomaly. We report the results for each parameterization as the mean \pm standard deviation of the RMSD determined from the Monte Carlo error analysis for both concentration and saturation anomaly (Table 3-1).

Unfortunately, the fact that the highest wind speeds in our time-series coincided with the upwelling event made it more difficult to discriminate between parameterizations. If the models are run through the full cruise duration, without resetting the concentrations after the upwelling, the four gas exchange parameterizations predict substantially different trajectories for He and Ne during and following the high wind event (Figure 3-5). As wind speed increases, the magnitudes of $F_{d}, F_{p}$, and $F_{c}$ increase and so do the differences between models. Below, we discuss the advantages and disadvantages of including upwelling versus not including upwelling in our model, and the insights gained from both approaches. 
Table 3-1: Performance of the four parameterizations in simulating the surface $\mathrm{He}$ and $\mathrm{Ne}$ data, with and without adjustment for upwelling. RMSD is reported as the mean \pm standard deviation, from the Monte Carlo error analysis. The \% best fit indicates the frequency that a specific parameterization gave the best fit (lowest RMSD) of the four parameterizations.

\begin{tabular}{lllll}
\hline & \multicolumn{3}{c}{ He, with upwelling adjustment } & \\
Parameterization & $\begin{array}{l}\text { He conc. RMSD } \\
\left(10^{-9} \text { mol kg } \mathrm{kg}^{-1}\right)\end{array}$ & $\begin{array}{l}\text { RMSD }(\%) \\
\text { RMturation }\end{array}$ & $\begin{array}{l}\text { \% best fit, } \\
\text { He conc. }\end{array}$ & $\begin{array}{l}\text { \% best fit, } \\
\text { He saturation }\end{array}$ \\
\hline Sweeney 2007 & $8.3 \pm 0.8$ & $0.47 \pm 0.05$ & 8 & 3 \\
Nicholson 2011 & $8.5 \pm 1.1$ & $0.46 \pm 0.05$ & 25 & 30 \\
Stanley 2009 & $10.4 \pm 1.5$ & $0.56 \pm 0.07$ & 2 & 3 \\
Liang 2013 & $7.9 \pm 0.7$ & $0.44 \pm 0.04$ & 65 & 64 \\
& & & & \\
\hline
\end{tabular}

Ne, with upwelling adjustment

\begin{tabular}{lllll} 
Parameterization & $\begin{array}{l}\text { Ne conc. RMSD } \\
\left(10^{-8} \mathrm{~mol} \mathrm{~kg}^{-1}\right)\end{array}$ & $\begin{array}{l}\text { Ne saturation } \\
\text { RMSD }(\%)\end{array}$ & $\begin{array}{l}\% \text { best fit, } \\
\text { Ne conc. }\end{array}$ & $\begin{array}{l}\% \text { best fit, } \\
\text { Ne saturation }\end{array}$ \\
\hline Sweeney 2007 & $3.8 \pm 0.4$ & $0.48 \pm 0.05$ & 2 & 1 \\
Nicholson 2011 & $3.8 \pm 0.4$ & $0.48 \pm 0.06$ & 32 & 33 \\
Stanley 2009 & $4.2 \pm 0.6$ & $0.55 \pm 0.08$ & 10 & 8 \\
Liang 2013 & $3.7 \pm 0.3$ & $0.46 \pm 0.04$ & 56 & 58
\end{tabular}

He, without upwelling adjustment

\begin{tabular}{|c|c|c|c|c|}
\hline Parameterization & $\begin{array}{l}\text { He conc. RMSD } \\
\left(10^{-9} \mathrm{~mol} \mathrm{~kg}^{-1}\right)\end{array}$ & $\begin{array}{l}\text { He saturation } \\
\text { RMSD (\%) }\end{array}$ & $\begin{array}{l}\% \text { best fit, } \\
\text { He conc. }\end{array}$ & $\begin{array}{l}\% \text { best fit, } \\
\text { He saturation }\end{array}$ \\
\hline Sweeney 2007 & $9.6 \pm 0.3$ & $0.53 \pm 0.02$ & 0 & 0 \\
\hline Nicholson 2011 & $7.8 \pm 0.6$ & $0.43 \pm 0.04$ & 45 & 36 \\
\hline Stanley 2009 & $13.7 \pm 0.8$ & $0.77 \pm 0.08$ & 0 & 0 \\
\hline Liang 2013 & $7.7 \pm 0.2$ & $0.42 \pm 0.01$ & 55 & 64 \\
\hline
\end{tabular}

\begin{tabular}{lllll}
\hline & \multicolumn{4}{l}{ Ne, without upwelling adjustment } \\
Parameterization & Ne conc. RMSD & Ne saturation & $\%$ best fit, & $\%$ best fit, \\
& $\left.\left(10^{-8} \mathrm{~mol} \mathrm{~kg}\right)^{-1}\right)$ & RMSD $(\%)$ & Ne conc. & Ne saturation \\
\hline Sweeney 2007 & $7.6 \pm 0.4$ & $0.96 \pm 0.05$ & 0 & 0 \\
Nicholson 2011 & $4.7 \pm 0.3$ & $0.58 \pm 0.04$ & 0 & 0 \\
Stanley 2009 & $3.4 \pm 0.1$ & $0.44 \pm 0.02$ & 100 & 100 \\
Liang 2013 & $6.8 \pm 0.4$ & $0.85 \pm 0.05$ & 0 & 0 \\
& & & & \\
\hline
\end{tabular}



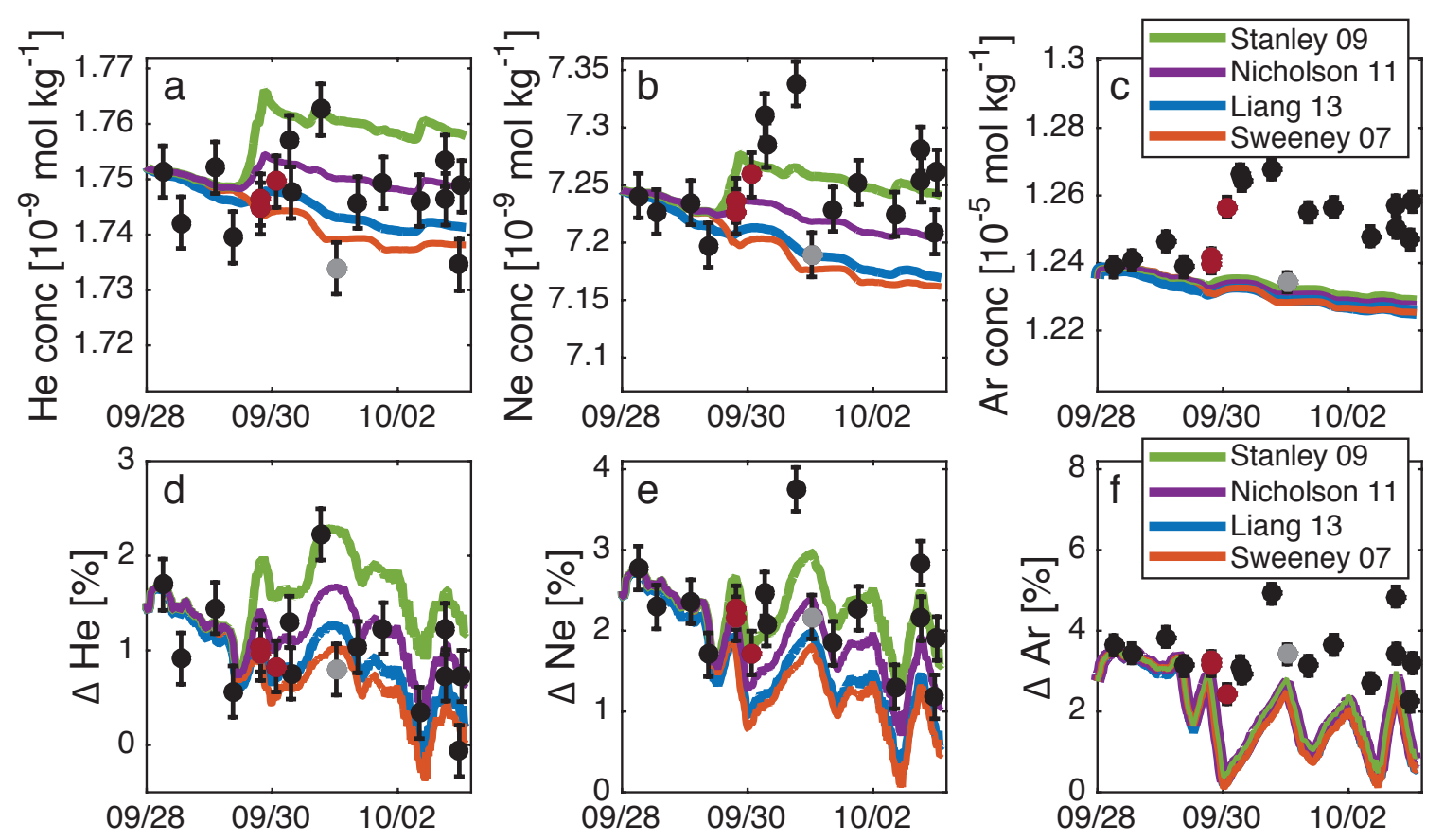

Figure 3-5: Model results without upwelling adjustment. Near-surface gas concentrations $(\mathrm{a}-\mathrm{c})$ and saturation anomalies $(\mathrm{d}-\mathrm{f})$, from samples and model results that were optimized to give the best fit to the first sample. All plotted samples were included in the error analysis.

\subsection{Discussion}

\subsubsection{Modeled surface concentrations and saturation anomalies}

When the model is adjusted for upwelling, the L13 model has the most skill in simulating the surface He and Ne data, yielding the lowest RMSDs of the four parameterizations (Table $3-1)$. L13 is the most accurate parameterization for predicting surface He concentration and saturation 65 and $64 \%$ of the time, respectively. Additionally, L13 is the most accurate parameterization for predicting surface Ne concentration and saturation 56 and $58 \%$ of the time, respectively. N11 is most accurate $25-33 \%$ of the time for He and Ne concentration and saturation. The S09 and Sw07 models were each the most accurate parameterization in $10 \%$ or less of the Monte Carlo simulations for He and Ne. In general, the RMSD for He and Ne concentration and saturation is similar for L13, N11, and Sw07, and higher for S09. The modeled concentrations and saturation anomalies for He and $\mathrm{Ne}$, when including upwelling, looked very similar for L13 and Sw07, although as shown in Table 3-1, L13 was usually the most accurate parameterization. However, a large part of the apparent skill of Sw07 at reproducing the observed He data comes from the upwelling adjustment. The gas added by upwelling compensates for the lack of gas added by an explicit bubble-generated flux.

For He, when the model is run without adjustment for upwelling, the differences between 
parameterizations, and the need for an explicit bubble-generated flux to produce the observed He supersaturation become clearer (Figure 3-5). Without the upwelling adjustment, the Sw07 parameterization predicts lower He concentrations and saturation anomalies than the other parameterizations, especially toward the end of the time-series. If the time-series were extended, the He concentration in Sw07 would continue to decay toward equilibrium $\left(H e_{e q}\right)$, and Sw07 would therefore underestimate the true He concentration. While we do not have a long enough time-series to say for certain, the fact that all but one surface He sample in our time-series are supersaturated suggests that a model that predicts equilibrium concentrations at steady state, such as Sw07, would be incorrect. An explicit bubble flux into the ocean is needed to generate consistently supersaturated surface waters for gases that are insensitive to temperature change. The solubility of He only changes by $0.2 \%{ }^{\circ} \mathrm{C}^{-1}$.

Without the upwelling adjustment, L13 is most accurate for predicting surface He concentration and saturation anomaly 55 and $64 \%$ of the time, and N11 is most accurate 45 and $36 \%$ of the time, respectively. The performance of L13 and N11 for He is similar with and without the upwelling adjustment. However, notably, without the upwelling adjustment, the S09 parameterization appears to overestimate the bubble flux for He during the high winds that coincided with the upwelling.

For Ne, when the model is run without an adjustment for upwelling (Figure 3-5), S09 is the most accurate parameterization for Ne concentration and saturation anomaly $100 \%$ of the time and has the lowest RMSDs of the four parameterizations, even though S09 has the highest RMSDs for Ne when the upwelling adjustment is included, and the highest RMSDs for He without the upwelling adjustment. This result suggests that Ne is an intermediate case, where upwelling, bubble-mediated fluxes, and diffusive fluxes are all important in setting the near-surface gas concentration. In the model runs without upwelling, S09 predicts high bubble fluxes which increase the Ne concentration to be similar to the true concentration after upwelling. The case with upwelling is likely more reasonable for Ne, although it does unfortunately overestimate the skill of the Sw07 model, due to the short duration of the time-series. Again, if the time-series were extended, the Ne concentration modeled with Sw07 would decay toward equilibrium, which is not consistent with the observations. All of the surface Ne samples are supersaturated, despite the solubility of Ne having a weak dependence on temperature $\left(0.7 \%{ }^{\circ} \mathrm{C}^{-1}\right)$, which supports the need for an explicit bubblemediated flux into the ocean to generate some portion of the observed supersaturation.

For Ar, Kr, and Xe, all parameterizations simulate the data well after the correction for upwelling. For these gases, the RMSD of each parameterization is very similar and we conclude that all parameterizations have similar skill in simulating the heavier noble gases for our dataset. With a somewhat longer time-series, and/or without the upwelling event, the differences between the parameterizations would likely be clearer, and we might be able to see the importance of bubble-mediated exchange for somewhat more soluble gases, such 
as Ar. When the model is run without adjustment for upwelling, all four parameterizations predict similar trajectories for $\mathrm{Ar}, \mathrm{Kr}$, and $\mathrm{Xe}$ and underestimate the concentrations and saturation anomalies of these gases after the upwelling event (Figure 3-5).

These results demonstrate the different factors that controlled each gas' change in concentration overnight on Sept 29-30. For He, bubble-mediated exchange generated a small concentration increase $(<1 \%)$. For Ne, upwelling and bubble-mediated exchange were both important in producing a moderate concentration increase $(\sim 1 \%)$. For $\mathrm{Ar}, \mathrm{Kr}$, and $\mathrm{Xe}$, upwelling/mixing caused a large (2-3\%) concentration increase. Because Ar, Kr, and Xe were barely affected by the high wind event that coincided with the upwelling, their concentrations could not be used to constrain the proportion of the surface water that was replaced with colder water with higher gas concentrations.

Over the whole time-series, the surface concentrations of $\mathrm{Ar}, \mathrm{Kr}$, and Xe were primarily controlled by upwelling and diffusive gas exchange. These more soluble gases contrast with He which was primarily controlled by bubble-mediated and diffusive gas exchange, and $\mathrm{Ne}$, which was controlled by a combination of upwelling, bubble-mediated gas exchange, diffusive gas exchange.
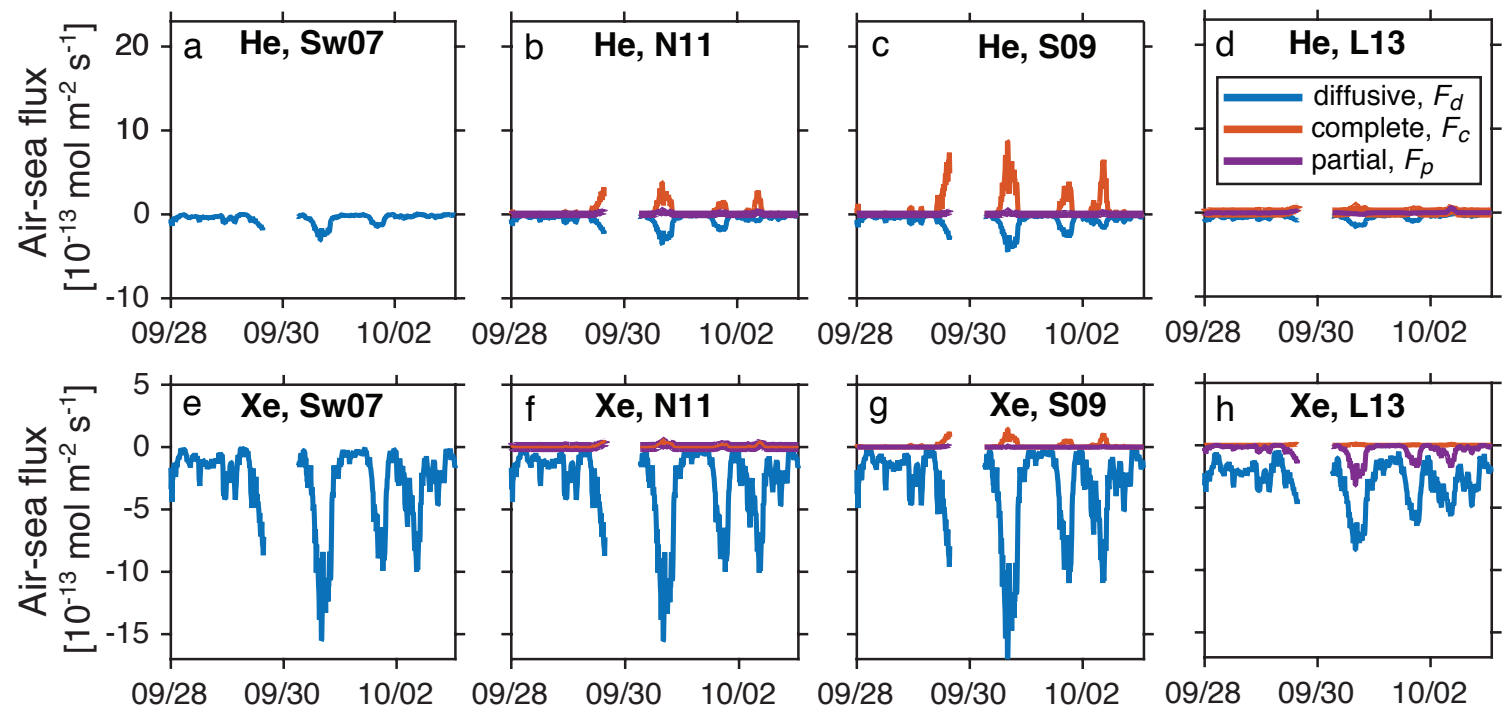

Figure 3-6: Air-sea gas fluxes of $\mathrm{He}(\mathrm{a}-\mathrm{d})$ and $\mathrm{Xe}(\mathrm{e}-\mathrm{h})$ during the time-series including adjustment for upwelling and modeled using all four parameterizations. Positive fluxes are into the ocean. The flux is separated into three components: diffusive flux $\left(F_{d}\right)$, complete bubble trapping $\left(F_{c}\right)$, and partial bubble trapping $\left(F_{p}\right)$. The Sw07 parameterization only includes $F_{d}$.

\subsubsection{Modeled air-sea fluxes}

For each gas, the differences between parameterizations, or lack thereof, can be understood by examining the gas fluxes predicted by each parameterization (Figure 3-6 and Table 3-2). 
The parameterizations differ greatly in their prediction of the total bubble flux, and the fraction of the total bubble flux from partial trapping versus complete trapping. The Sw07 and N11 parameterizations use the same equation for $F_{d}$, but Sw07 does not include the bubble terms $F_{c}$ and $F_{p}$. The magnitude of $F_{d}$ for He is $19 \%$ less in Sw07 compared to N11 because there is no bubble-mediated exchange to increase the saturation anomaly, and thus the diffusive flux of He. The magnitude of the He bubble flux, $F_{b}$, is greatest for S09 (double N11 and twelve times L13); thus S09 predicts the highest He concentrations throughout the time-series.

The lower skill of the S09 parameterization compared to N11 and L13 in simulating He (both with and without the upwelling adjustment), and the improved performance of S09 in simulating Ne when the upwelling adjustment is not performed, both suggest that S09 may be overestimating bubble-mediated exchange in this environment. One potential explanation is that the S09 parameterization was tuned using He solubility data that was $\sim 1 \%$ too low (saturation anomalies that were $\sim 1 \%$ too high), compared to the LJ15 solubility used in this model. Assuming that the S09 model was fit to reproduce He saturation anomalies that were too high, it may overestimate bubble fluxes. As we observe, S09 does predict the largest bubble-mediated fluxes of the four parameterizations. When we run the model using the He solubility of Weiss (1971), which is $\sim 2 \%$ lower than the He solubility of LJ15, all models underestimate the near-surface He concentrations and saturation anomalies (Figures B-2 and B-3 in Supplemental Information). The S09 parameterization comes closest to simulating the He data when using the Weiss (1971) solubility because it predicts the largest bubble fluxes.

The N11 and L13 parameterizations were fit to inert gas data not including He, and the L13 parameterization was not tuned using any oceanic gas data. Therefore, N11 and L13 would not have errors related to uncertainties in He solubility. This result underscores the need for accurate gas solubility functions in order to realistically interpret oceanic gas data (Hamme and Emerson, 2004a).

The modeled air-sea fluxes help to explain why the parameterizations all give very similar trajectories for Xe, Kr, and Ar in Figure 3-4. For Xe, the Sw07 and N11 models predict nearly the same $F_{d}$ because the diffusive flux greatly exceeds the bubble flux (Table 3-2). The absolute and relative values of $F_{c}$ and $F_{p}$ for Xe vary widely between the different parameterizations. L13 is unique among the parameterizations in simulating a bubble stripping effect in which heating-induced supersaturation exceeds the bubble overpressure effect, resulting in a net removal of gas by partial bubble trapping $\left(F_{p}\right)$. The S09 and N11 parameterizations always give net bubble flux into the ocean for $F_{p}$, even for supersaturated gases. N11, S09, and Sw07 predict similar $F_{d}$ for Xe and have a small or zero bubble flux (the magnitude of $F_{b}$ is $5 \%$ or less of the magnitude of $F_{t}$ ). For L13, the sum of $F_{d}$ and $F_{b}$ for Xe gives a net flux that is very similar to $\mathrm{F}_{d}$ for the other parameterizations. 
Table 3-2: Comparison of air-sea fluxes, based on data plotted in Figure 3-6 (i.e., including adjustment for upwelling). The $F_{d}$ and $F_{b}$ are mean values, averaged over the whole timeseries. Positive fluxes are into the ocean. Liang 2013 predicts $F_{p}<0$ and $F_{c}>0$ for He and Xe. Therefore, the fraction of $F_{b}$ from $F_{p}$ is negative for $\mathrm{He}\left(F_{b}>0\right)$ and over $100 \%$ for Xe $\left(F_{b}<0\right)$.

\begin{tabular}{|c|c|c|c|}
\hline \multicolumn{4}{|c|}{ Helium fluxes } \\
\hline Parameterization & $\begin{array}{l}F_{d} \\
{\left[10^{-1}\right.}\end{array}$ & $\begin{array}{l}F_{b} \\
\left.\mathrm{~mol} \mathrm{~m}^{-2} \mathrm{~s}^{-1}\right]\end{array}$ & $\begin{array}{l}\text { Fraction of } F_{b} \text { from } F_{p} \\
{[\%]}\end{array}$ \\
\hline Sweeney 2007 & -4.7 & 0 & - \\
\hline Nicholson 2011 & -5.8 & 3.7 & 11 \\
\hline Stanley 2009 & -7.5 & 7.9 & 3 \\
\hline Liang 2013 & -5.0 & 0.6 & -16 \\
\hline \multicolumn{4}{|c|}{ Xenon fluxes } \\
\hline Parameterization & $\begin{array}{l}F_{d} \\
{\left[10^{-1}\right.}\end{array}$ & $\begin{array}{l}F_{b} \\
\left.\mathrm{~mol} \mathrm{~m}^{-2} \mathrm{~s}^{-1}\right]\end{array}$ & $\begin{array}{l}\text { Fraction of } F_{b} \text { from } F_{b} \\
{[\%]}\end{array}$ \\
\hline Sweeney 2007 & -2.7 & 0 & - \\
\hline Nicholson 2011 & -2.7 & 0.1 & 41 \\
\hline Stanley 2009 & -3.0 & 0.1 & 1 \\
\hline Liang 2013 & -2.8 & -0.4 & 103 \\
\hline
\end{tabular}

The choice of optimizing the initial conditions to concentration versus saturation anomaly does not significantly affect our interpretations, as shown in Table 3-1. For example, the modeled total air-sea flux $\left(F_{t}\right)$ for each gas varies by $7 \%$ or less between the two optimization values. Additionally, the Monte Carlo error analysis results are very similar when the wind speed from the propeller anemometer is used rather than the sonic anemometer. The modeled $F_{t}$ for each parameterization is generally within $13 \%$ for either anemometer, and both wind speed sensors predict L13 is most accurate $64-77 \%$ of the time for He and 56-72 $\%$ of the time for Ne.

\subsection{Conclusions and future work}

This study is complementary to others that have demonstrated the value of using oceanic inert gas measurements in tandem with models to quantify air-sea gas exchange fluxes (Nicholson et al., 2011; Stanley et al., 2009b; Hamme and Emerson, 2006). We demonstrated that short-term, high-frequency measurements of inert gases and diapycnal diffusivity can be used to quantify air-sea gas exchange in coastal regions. We found that accurately parameterizing bubble-mediated exchange was necessary to simulate the near-surface measurements of $\mathrm{He}$ and Ne collected during our time-series. The tested parameterizations gave a wide range of results for the direction and magnitude of the net bubble flux, and the proportion of partial versus complete bubble trapping, indicating that there are still large uncertainties 
in models of bubble-mediated gas exchange. Higher wind speed conditions, and/or a longer period of Lagrangian observations would have resulted in a greater divergence between the parameterizations for He and Ne.

The parameterizations that displayed the most skill in simulating the He observations were Liang 2013 (Liang et al., 2013) followed by Nicholson 2011 (Nicholson et al., 2011). This result was observed regardless of whether we ran the model with or without an adjustment for upwelling. For Ne, the parameterization of L13 was most accurate when the model was adjusted for upwelling partway through the cruise. For $\mathrm{Ar}, \mathrm{Kr}$, and $\mathrm{Xe}$, all parameterizations gave very similar results and simulated the observations well, after adjustment for upwelling. For these heavier gases and the moderate wind speeds observed during our study, we conclude that diffusive exchange driven by temperature change was more important than bubble-mediated fluxes in controlling the gas concentrations of $\mathrm{Ar}, \mathrm{Kr}$, and $\mathrm{Xe}$. Due to the complication of upwelling during our cruise, a longer uninterrupted Lagrangian time-series and/or higher average wind speeds would have enabled clearer discrimination between parameterizations for the heavier gases, and an evaluation of the importance of bubble-mediated exchange for Ar.

The four tested parameterizations performed fairly well, despite not explicitly incorporating factors other than wind speed that may affect gas exchange rates in the coastal ocean (e.g., fetch and surfactant concentration). Evaluating these and other gas exchange parameterizations against in situ measurements in a wide range of environmental conditions, and for longer periods of time, is an important goal of future work.

\subsection{Acknowledgments}

We thank Captain Andrew McKee of the R/V Western Flyer and all cruise participants (crew and science team) for a successful cruise. Mooring data were collected and made available by the Biological Oceanography Group (PI: Francisco Chavez) at the Monterey Bay Aquarium Research Institute. Noble gas sample extraction, analysis, and data reduction was performed by Kevin Cahill, Bill Jenkins, and Dempsey Lott of the Woods Hole Oceanographic Institution Isotope Geochemistry Facility. We also thank one anonymous reviewer for their thoughtful comments that improved the manuscript. Funding for this work was provided by NSF award OCE-1060840 to RHR Stanley, NSF award OCE-1129644 to DP Nicholson, an Ocean Ventures Fund award from the WHOI Academic Programs Office to CC Manning, and graduate scholarships from NSERC and CMOS to CC Manning. 


\subsection{Supplemental information}

The Supplemental Information for this paper includes the noble gas data presented in the paper and plots of the model results using published solubility functions for $\mathrm{He}, \mathrm{Kr}$, and Xe, and the gas_toolbox, a set of MATLAB functions for calculating gas exchange fluxes with the parameterizations cited in this paper. The gas_toolbox is freely available online at http://www.github.com/dnicholson/gas_toolbox (Manning and Nicholson, 2016), and the remainder of the Supplemental Information is in Appendix B. Use of the gas_toolbox also requires installation of the Gibbs-Seawater Oceanographic Toolbox for MATLAB (http://www.teos-10.org/software.htm) (McDougall and Barker, 2011). 


\section{Chapter 4}

\section{Impact of recently upwelled water on productivity investigated using in situ and incubation-based methods in Monterey Bay}

This chapter was submitted to the Journal of Geophysical Research: Oceans on September 2, 2016 and a revised manuscript has been requested by the editor.

Cara C. Manning, Rachel H. R. Stanley, David P. Nicholson, Jason M. Smith, J. Timothy Pennington, Melanie R. Fewings, Michael E. Squibb, and Francisco P. Chavez (2016) Impact of recently upwelled water on productivity investigated using in situ and incubationbased methods in Monterey Bay. In revision for J. Geophys. Res. Oceans, manuscript 2016JC012306.

The supplemental information for this chapter can be found in Appendix C. 


\subsection{Abstract}

Photosynthetic conversion of $\mathrm{CO}_{2}$ to organic carbon and its transport from the surface to the deep ocean is an important regulator of atmospheric $\mathrm{CO}_{2}$. To understand the controls on carbon fluxes in a productive region impacted by upwelling, we measured biological productivity via multiple methods during a cruise in Monterey Bay, California. We quantified net community production and gross primary production from measurements of $\mathrm{O}_{2} / \mathrm{Ar}$ and the triple oxygen isotopic composition of $\mathrm{O}_{2}\left({ }^{17} \Delta\right)$, respectively. We simultaneously conducted incubations measuring uptake of ${ }^{14} \mathrm{C},{ }^{15} \mathrm{NO}_{3}^{-}$and ${ }^{15} \mathrm{NH}_{4}^{+}$, and deployed shallow particle interceptor traps. At the start of the cruise (Phase 1) the productivity methods agreed well, with net community production of 36(10) and $37(8) \mathrm{mmol} \mathrm{C} \mathrm{m}^{-2} \mathrm{~d}^{-1}$ from $\mathrm{O}_{2} / \mathrm{Ar}$ and $\mathrm{NO}_{3}^{-}$uptake respectively, net primary production of $90(22) \mathrm{mmol} \mathrm{C} \mathrm{m}^{-2} \mathrm{~d}^{-1}$ from $\mathrm{C}$ uptake, and gross primary production of $258(28) \mathrm{mmol} \mathrm{C} \mathrm{m}^{-2} \mathrm{~d}^{-1}$ from ${ }^{17} \Delta$. Later in the cruise (Phase 2), recently upwelled water with higher nutrient concentrations entered the study area, causing ${ }^{14} \mathrm{C}$ and ${ }^{15} \mathrm{NO}_{3}^{-}$uptake to increase substantially. Continuous $\mathrm{O}_{2} / \mathrm{Ar}$ measurements revealed submesoscale variability in productivity in Phase 2 that was not evident from the incubations. These results demonstrate the insights gained by using multiple methods to quantify productivity in a dynamic system.

\subsection{Introduction}

Although microbial carbon uptake in the surface ocean plays a major role in regulating atmospheric $\mathrm{CO}_{2}$ levels, quantifying the rate of this process has proven challenging on both a local and a global scale (Emerson, 2014; Siegel et al., 2016). Through photosynthesis, microbes in the sunlit euphotic zone convert $\mathrm{CO}_{2}$ to organic carbon (OC), simultaneously consuming nutrients and producing $\mathrm{O}_{2}$ (Longhurst and Harrison, 1989; Ducklow et al., 2001). The majority of the $\mathrm{OC}$ is respired back to $\mathrm{CO}_{2}$ by autotrophs and heterotrophs within the euphotic zone, but some small fraction of the OC is exported to deeper depths in the ocean, where it is isolated from the atmosphere on timescales ranging from weeks to millennia (Ducklow et al., 2001; Emerson, 2014). The magnitude of this biological pump, and the mechanisms controlling its spatial and temporal variability, are thus of great importance for accurately modeling the global carbon cycle and predicting its future changes (Falkowski et al., 1998; Behrenfeld et al., 2005; Ciais et al., 2013).

In this paper, we refer to various aspects of the ecosystem metabolism related to the biological pump (Williams, 1993). Gross primary production (GPP) is the total amount of carbon fixed by autotrophic microbes into organic carbon. Net primary production (NPP) is GPP minus autotrophic respiration $\left(R_{A}\right)$, i.e. respiration by phytoplankton only. NPP quantifies the amount of carbon available to the heterotrophic community. Net community 
production $(\mathrm{NCP})$ is NPP minus heterotrophic respiration $\left(R_{H}\right)$, or equivalently, GPP minus community respiration (equations 4.1 and 4.2 ):

$$
\begin{gathered}
N C P=N P P-R_{H} \\
N C P=G P P-R_{A}-R_{H}
\end{gathered}
$$

NCP quantifies the carbon available for export from the euphotic zone, and therefore NCP is the key parameter in estimating the magnitude of the biological pump (Laws, 1991; Williams and Purdie, 1991; Hansell and Carlson, 1998). We also use the terms gross oxygen production (GOP) to refer to the gross amount of $\mathrm{O}_{2}$ produced by photosynthesis, and net oxygen production (NOP) to refer to the net amount of $\mathrm{O}_{2}$ produced (Munro et al., 2013). GOP and NOP can be converted to GPP and NCP respectively, based on empirically-derived stoichiometric ratios of O:C for gross and net production (Laws, 1991; Bender et al., 1999). In this study, we quantify all five parameters: GPP, NPP, NCP, $R_{A}$ and $R_{H}$, for the mixed layer, enabling us to estimate the amount of inorganic carbon that is fixed into organic carbon, the amount of organic carbon available for transport out of the mixed layer, and the importance of heterotrophs and autotrophs for recycling carbon within the mixed layer.

Several techniques are available for estimating GPP, NPP, and NCP, including bottle incubations, in situ mass balance techniques, and algorithms derived from satellite-based ocean color data (Emerson et al., 1997; Behrenfeld et al., 2005; Emerson, 2014). Each method measures a specific aspect of the ecosystem metabolism, integrates over a specific timescale, and is thought to have inherent advantages and disadvantages (Williams et al., 2004; Juranek and Quay, 2013; Emerson, 2014). However, in many studies, only one or two methods of estimating productivity are used, making it challenging or impossible to evaluate possible method biases and to establish quantitative relationships between productivity estimates from different methods.

The cruise took place in the Monterey Bay, a highly productive embayment on the central California coast, approximately $20 \mathrm{~km}$ long and $30 \mathrm{~km}$ wide (Pilskaln et al., 1996; Pennington and Chavez, 2000). The bay is within an eastern boundary upwelling zone and is affected by wind-driven coastal upwelling, which occurs most intensely just north of the bay at Point Año Nuevo (Rosenfeld et al., 1994; Graham and Largier, 1997; Woodson et al., 2009). Filaments of cold upwelled water tend to be advected southward from the Point into the middle of the bay, our main study area (Ryan et al., 2009). The large-scale upwellingfavorable winds are most intense in spring and summer but continue periodically into the fall, when this cruise took place (Graham and Largier, 1997). These upwelling regions play a disproportionately large role in the global carbon cycle, relative to their small spatial extent (MacIsaac et al., 1985; Falkowski et al., 1998; Pennington et al., 2006). In these regions, nutrient-rich water is transported from deeper waters to the euphotic zone, stimulating 
biological activity. High productivity at the base of the food web sustains enhanced biomass at higher trophic levels, enabling these regions to support active fisheries (Peterson et al., 1988).

Monterey Bay has been well-studied with respect to biogeochemistry. Monthly, multiyear time-series of productivity, based on incubations measuring the uptake of ${ }^{14} \mathrm{C}$-labeled dissolved inorganic carbon (DIC) and ${ }^{15} \mathrm{NO}_{3}^{-}$have been reported previously (Pennington and Chavez, 2000; Wilkerson et al., 2000), and numerous process-oriented biological studies have been conducted (Pilskaln et al., 1996; Kudela and Dugdale, 2000; Ward, 2005; Ryan et al., 2009; Johnson, 2010; Smith et al., 2014a, 2016). However, productivity estimates derived from in situ measurements of $\mathrm{O}_{2} / \mathrm{Ar}$ and the triple oxygen isotopic composition of $\mathrm{O}_{2}$ have not been published for Monterey Bay.

The in situ $\mathrm{O}_{2}$ mass balance technique has been widely applied for estimating NCP and GPP in the open ocean (Juranek and Quay, 2013), but can be challenging to apply in systems where recently-upwelled water is observed at the surface; this low $\mathrm{O}_{2}$ water may bias estimates low if not accurately accounted for (Munro et al., 2013; Teeter, 2014; Haskell et al., 2016b). Furthermore, inferring carbon export below the mixed layer from techniques within the mixed layer is complicated by the fact that lateral transport of surface waters and oceanic fronts may cause a spatial and/or temporal decoupling between carbon fixation and export (Olivieri and Chavez, 2000; Estapa et al., 2015; Nagai et al., 2015).

Here we present one of the first published data sets where productivity estimates from both $\mathrm{O}_{2} / \mathrm{Ar}$ and the triple oxygen isotope composition of $\mathrm{O}_{2}\left({ }^{17} \Delta\right)$ are combined with multiple other methods including incubations measuring the uptake of ${ }^{14} \mathrm{C},{ }^{15} \mathrm{NO}_{3}^{-}$and ${ }^{15} \mathrm{NH}_{4}^{+}$ and nitrification (microbial oxidation of $\mathrm{NH}_{4}^{+}$to $\mathrm{NO}_{3}^{-}$), as well as sediment trap-based fluxes of particulate carbon and nitrogen (PC and PN). For example, there are few published studies where ${ }^{15} \mathrm{NO}_{3}^{-}$uptake (new production) and in situ $\mathrm{O}_{2} / \mathrm{Ar}$ (net community production) were measured simultaneously at the same locations (Emerson et al., 1993; Giesbrecht et al., 2012; Hamme et al., 2012). Although theoretically these methods should be equivalent at steady state and/or when averaged over large spatiotemporal scales (Laws, 1991; Falkowski et al., 2003), this assumption has been tested at a limited number of locations, primarily high nitrate, low chlorophyll open ocean regions such as the Southern Ocean and subarctic North Pacific (Hendricks et al., 2005; Reuer et al., 2007; Giesbrecht et al., 2012). In this study, we compare productivity estimates by multiple methods in a new environment, a nitrogen-limited, highly productive site (Kudela and Dugdale, 2000) within the California Current System. 


\subsection{Background on methods}

\subsection{1 ${ }^{14} \mathrm{C}$ incubations}

The ${ }^{14} \mathrm{C}$-method of estimating primary production (abbreviated herein as ${ }^{14} \mathrm{C}-\mathrm{PP}$ ) is one of the oldest and most widely used methods of quantifying marine productivity (Steemann Nielsen, 1951, 1952), and it is the standard against which many satellite-based productivity algorithms are validated and calibrated (Behrenfeld and Falkowski, 1997; Carr et al., 2006). However, there remains uncertainty regarding which aspects of the ecosystem metabolism the incubations measure. The degree to which the incubation captures gross versus net production depends on incubation duration, the time of day at which the incubation is initiated, the light level in each bottle, and even the ratio of photosynthesis to respiration itself (Steemann Nielsen and Jensen, 1957; Williams et al., 1983; Williams, 1993; Karl et al., 1996; Bender et al., 1999; Marra, 2002, 2009; Pei and Laws, 2013; Pennington et al., 2015). For example, a $12 \mathrm{~h}$ daytime-only incubation will yield higher ${ }^{14} \mathrm{C}-\mathrm{PP}$ than a $24 \mathrm{~h}$ incubation carried out over a full day-night solar cycle, because nighttime respiration will convert some of the OC produced during daytime back to DIC (Bender et al., 1999). There is generally agreement that $24 \mathrm{~h}{ }^{14} \mathrm{C}$ incubations initiated during daytime measure something between GPP and NCP (Smith et al., 1984; Bender et al., 1999; Juranek and Quay, 2005; Marra, 2002, 2009; Juranek and Quay, 2013).

Another important aspect of the method is that the procedure only measures the carbon that is retained on the filter. The $\mathrm{GF} / \mathrm{F}$ filters retain ${ }^{14} \mathrm{C}-\mathrm{POC}$, and some fraction of the ${ }^{14} \mathrm{C}-\mathrm{DOC}$ that is exuded by phytoplankton (Karl et al., 1998). Some of this ${ }^{14} \mathrm{C}-\mathrm{DOC}$ is taken up by bacteria which are then retained on the filter. Of the labeled material that remains as DOC when the incubation is terminated, some passes through the filter and some is adsorbed (Karl et al., 1998). The fraction of total OC fixed that is lost as DOC and not retained on the filter can be up to $30 \%$ (Karl et al., 1998). Thus, the incubations underestimate the total ${ }^{14} \mathrm{C}$ uptake into $\mathrm{OC}$.

\subsection{2 ${ }^{15} \mathrm{~N}$ incubations}

Nitrogen is often a limiting nutrient in marine ecosystems, including Monterey Bay (Kudela and Dugdale, 2000). By measuring uptake of individual N species and nitrification we can better understand the role that nutrient availability plays in regulating primary production.

Phytoplankton assimilate various forms of inorganic nitrogen through processes indirectly related to photosynthesis (Dugdale and Goering, 1967). When these microbes die and/or are eaten, $\mathrm{PN}$ and $\mathrm{NH}_{4}^{+}$(reduced N) are released. Some of the PN sinks below the euphotic zone, and is remineralized to $\mathrm{NH}_{4}^{+}$. Over annual time scales, the vast majority of $\mathrm{NH}_{4}^{+}$below the euphotic zone is oxidized to $\mathrm{NO}_{3}^{-}$by nitrifying organisms. This $\mathrm{NO}_{3}^{-}$is 
then transferred back to the euphotic zone through upwelling and mixing processes. Within this paradigm, $\mathrm{NH}_{4}^{+}$-based production is called regenerated production (as $\mathrm{NH}_{4}^{+}$is produced within the euphotic zone) and $\mathrm{NO}_{3}^{-}$-based production is called new production (as $\mathrm{NO}_{3}^{-}$is produced below the euphotic zone). At steady state, i.e., if the concentration of $\mathrm{NO}_{3}^{-}$and organic matter within the euphotic zone is not changing, then new production will equal $\mathrm{NCP}$ and export production. If we assume that the only forms of nitrogen taken up by phytoplankton are $\mathrm{NO}_{3}^{-}$and $\mathrm{NH}_{4}^{+}$, then the sum of the uptake of these two species gives NPP (Dugdale and Goering, 1967), after conversion from N to C units based on the Redfield ratio or other C:N data (Redfield et al., 1963).

There are, of course, limitations to this approach. The method underestimates the total $\mathrm{N}$ uptake because the uptake of other forms of regenerated $\mathrm{N}$ such as DON (e.g., urea, amino acids, and proteins) is not quantified (McCarthy, 1972; Eppley and Peterson, 1979), and because some fraction of the ${ }^{15} \mathrm{~N}$ label that is taken up by phytoplankton and then exuded as DON is not retained on the filter. Another important consideration is that nitrification within the euphotic zone can generate a significant fraction of the total euphotic zone $\mathrm{NO}_{3}^{-}$ inventory in many oceanic regions (Dore and Karl, 1996; Diaz and Raimbault, 2000; Yool et al., 2007; Grundle et al., 2013; Santoro et al., 2013) including Monterey Bay (Ward, 2005; Smith et al., 2014a,b). In this study, we performed nitrification rate estimates to separate $\mathrm{NO}_{3}^{-}$produced within the euphotic zone from $\mathrm{NO}_{3}^{-}$produced below the euphotic zone, for the purpose of calculating new production (Yool et al., 2007).

An additional methodological challenge is that $\mathrm{N}$ occurs at low concentrations in much of the world's oceans (Moore et al., 2013), including Monterey Bay (Kudela and Dugdale, 2000), and therefore the addition of bioavailable nitrogen could perturb nutrient cycling within the incubation bottle. Ideally, the concentration of tracer added should be $\leq 10 \%$ of the ambient concentration, to ensure that the tracer itself does not stimulate $\mathrm{N}$ assimilation and primary production (Dugdale and Goering, 1967).

\subsection{3 $\quad \mathrm{O}_{2}$ mass balance}

Measurements of $\mathrm{O}_{2} / \mathrm{Ar}$ gas ratios and the triple oxygen isotopic composition of $\mathrm{O}_{2}$ are effective tracers of NCP and GPP, respectively. The $\mathrm{O}_{2} / \mathrm{Ar}$ mass balance technique has become a widely used approach for in situ determinations of NCP. Both gases have similar diffusivity, solubility, and dependence of solubility on temperature (Ferrell and Himmelblau, 1967; Jähne et al., 1987a; Garcia and Gordon, 1992; Hamme and Emerson, 2004a). As a result, $\mathrm{Ar}$ is commonly used as an abiotic analogue for $\mathrm{O}_{2} ; \mathrm{Ar}$ responds similarly to physical forcings but has no biological flux (Craig and Hayward, 1987; Spitzer and Jenkins, 1989). To calculate net biological production of $\mathrm{O}_{2}$, investigators must correct for the effect of physical processes because in many environments the physical and biological fluxes of $\mathrm{O}_{2}$ are similar in magnitude in the mixed layer (Emerson, 1987; Hamme and Emerson, 2006; 
Castro-Morales and Kaiser, 2012; Giesbrecht et al., 2012). Physical processes that affect $\mathrm{O}_{2}$ include air-sea gas exchange, vertical mixing/entrainment and lateral advection.

The triple oxygen isotopic tracer of dissolved $\mathrm{O}_{2}$ exploits the unique isotopic signatures of $\mathrm{O}_{2}$ produced by photosynthesis, consumed by respiration, and added to the ocean by air-sea gas exchange (Luz and Barkan, 2000, 2005; Juranek and Quay, 2013). Specifically, photochemical reactions in the upper atmosphere impart a small mass-independent isotopic fractionation signature on tropospheric $\mathrm{O}_{2}$, which is transferred to $\mathrm{O}_{2}$ dissolved in the ocean through air-sea gas exchange (Thiemens et al., 1995; Luz and Barkan, 2000), with a known mass-dependent fractionation (Reuer et al., 2007; Stanley et al., 2010). Photosynthetic $\mathrm{O}_{2}$ is derived from $\mathrm{H}_{2} \mathrm{O}$, which does not have a measurable mass-independent isotopic signature (Luz and Barkan, 2000; Juranek and Quay, 2013). Respiratory isotopic fractionation of $\mathrm{O}_{2}$ has been determined experimentally (Luz and Barkan, 2005).

In many studies, each location is only sampled once (e.g. transect cruises) or sampling at the same location occurs very far apart in time (much longer than the residence time of $\mathrm{O}_{2}$ in the mixed layer, which is typically on the order of two weeks), making it necessary to assume the gases are at steady state in order to calculate NCP and GPP (Stanley et al., 2010; Giesbrecht et al., 2012; Munro et al., 2013). When a higher-frequency time-series of measurements is obtained (as occurred during this cruise), investigators can quantify the change in $\left[\mathrm{O}_{2}\right]$ and ${ }^{17} \Delta$ with time and include these terms in the productivity estimates when appropriate (Hamme et al., 2012; Tortell et al., 2014; Wilson et al., 2015). Because the calculations of $\mathrm{NCP}$ and GPP from $\mathrm{O}_{2} / \mathrm{Ar}$ and triple oxygen isotope data can vary substantially between studies, we outline our calculations in section 4.5.

\subsubsection{Sediment traps}

Sediment traps directly measure the flux of organic particles out of the surface ocean, in contrast to the previously described methods which measure processes within the surface ocean and are used to infer downward organic matter flux (Buesseler, 1991; Emerson, 2014). To interpret the sediment trap data, we must keep in mind that there are three main modes of OC export: passive sinking of particles, active transport by zooplankton and other animals who consume OC near the surface and exude it at depth, and physical transport of OC by mixing and advection (Buesseler, 1991; Carlson et al., 1994; Steinberg et al., 2000; Emerson, 2014). Sediment traps primarily capture the passively sinking particulate flux. However, of the methods used during this cruise, sediment traps are the only one that measures any component of the export flux directly, and the only one that quantifies the $\mathrm{C}$ flux below the euphotic zone.

A challenge in the use and interpretation of data from upper ocean particle traps, which are intended to collect the passively sinking flux of detrital matter, is the inadvertent collection of actively swimming zooplankton in the trap (Michaels et al., 1990). These living 
organisms can be a significant portion of the carbon content retained on the filter but do not represent carbon export. Various correction methods for zooplankton swimmers can be used, including placing screens within the particle traps to prevent zooplankton from sinking to the bottom, counting zooplankton within the unfiltered trap water and/or manually removing zooplankton from the filter paper after filtration (Michaels et al., 1990).

\subsection{Field and analytical methods}

\subsubsection{Cruise description}

The cruise (CANON14) took place from 27 September-3 October 2014. During the cruise, we obtained casts with a conductivity-temperature-depth sensor (CTD, Sea-Bird SBE 9) and Niskin bottle rosette to $\sim 180 \mathrm{~m}$ four times per day, at roughly 06:00, 12:00, 18:00, and 00:00 local time. Throughout the paper, all dates and times are reported as local time (UTC - 07:00). All CTD casts at $6 \mathrm{~h}$ intervals were obtained near mooring 1 (M1, 36.75 ${ }^{\circ} \mathrm{N}, 122.03{ }^{\circ} \mathrm{W}, 21 \mathrm{~km}$ west of Moss Landing, CA), which is operated by the Monterey Bay Aquarium Research Institute. Sunrise and sunset were at 07:00 and 19:00, respectively. Immediately prior to nearly every cast, we obtained vertical profiles of microscale turbulence to $\sim 70 \mathrm{~m}$ using a vertical microstructure profiler (VMP-200, Rockland Scientific). Rates of turbulent kinetic energy dissipation were calculated using the profiler's shear probes and fast temperature sensor, and diapycnal dissipation and diffusivity $\left(K_{z}\right)$ were estimated following Wolk et al. (2002). These diffusivity measurements are used to quantify the impact of vertical mixing on the mass balance of the gas tracers (Manning et al., 2016c).

Throughout the cruise we deployed an autonomous underwater vehicle (AUV), which drifted at $\sigma_{\theta} \approx 25.2 \mathrm{~kg} \mathrm{~m}^{-3}(25-45 \mathrm{~m}$ depth$)$, near the base of the thermocline. We conducted CTD casts and deployed sediment traps in the vicinity of the drifting subsurface AUV; however, the ship's course between casts sometimes varied in order to meet other science goals (Figures C-1-C-2). We use wind speed measurements obtained by a Vaisala ultrasonic anemometer from M1 to calculate air-sea gas transfer coefficients $(k)$.

\subsubsection{Rates of ${ }^{14} \mathrm{C}$ uptake $\left({ }^{14} \mathrm{C}-\mathrm{PP}\right)$}

We collected water for in situ measurements of ${ }^{14} \mathrm{C}-\mathrm{PP}$ just prior to dawn each day from depths corresponding to $100,50,30,15,5,1$, and $0.1 \%$ surface irradiance, $\mathrm{I}_{o}$ (photosynthetically active radiation, or PAR), estimated using a Secchi disk. We conducted $24 \mathrm{~h}$ incubations at the seven in situ light levels, following the procedure in Pennington et al. (2015). For the depth-integrated ${ }^{14} \mathrm{C}-\mathrm{PP}$ (calculated using a trapezoidal integration), we calculated the simulated depth of each light level based on the algorithm of Morel and Berthon (1989). Each daily ${ }^{14} \mathrm{C}-\mathrm{PP}$ measurement has an error of $\pm 7 \%$ RSD (relative standard deviation), 
based on another data set of triplicate incubations by the authors (Pennington et al., 2015). During this cruise, a single incubation was carried out at each depth.

\subsubsection{Rates of ${ }^{15} \mathrm{NO}_{3}^{-}$uptake, ${ }^{15} \mathrm{NH}_{4}^{+}$uptake, and nitrification}

As for the ${ }^{14} \mathrm{C}-\mathrm{PP}$ incubations, we collected water for in situ measurements of ${ }^{15} \mathrm{NO}_{3}^{-}$uptake, ${ }^{15} \mathrm{NH}_{4}^{+}$uptake, and nitrification just before dawn each day from depths of 50, 30, 15, 5, 1, and $0.1 \% \mathrm{I}_{o}$. We followed the procedures of Kudela and Dugdale (2000) and Smith et al. (2014b) for the incubations. Two sets of incubations took place: one set included all light depths and was held for $24 \mathrm{~h}$; a second set from 50, 1, and $0.1 \%$ light were terminated after $6 \mathrm{~h}$ to assess the effects of incubation time and isotope dilution on rate estimates. We added isotopically labeled substrates to a final concentration of $0.2-1 \mu \mathrm{mol} \mathrm{L}-1$ of ${ }^{15} \mathrm{NO}_{3}^{-}$ and $0.08 \mu \mathrm{mol} \mathrm{L}{ }^{-1}$ of ${ }^{15} \mathrm{NH}_{4}^{+}$. For $\mathrm{NO}_{3}^{-}$, we targeted a $10 \%{ }^{15} \mathrm{~N}$ enrichment at the start of each incubation using an established relationship between temperature and $\left[\mathrm{NO}_{3}^{-}\right]$in Monterey Bay because nutrient analyses were not yet available at the time of incubation. For $\mathrm{NH}_{4}^{+}$, we added a fixed quantity of tracer to all bottles, which was selected to ensure ${ }^{15} \mathrm{~N}$ uptake was detectable, while also minimizing perturbation to the system over the three orders of magnitude range in $\left[\mathrm{NH}_{4}^{+}\right]$observed in Monterey Bay and the contiguous California Current System (Smith et al., 2014a, 2016). Post-cruise analysis indicates that average ${ }^{15} \mathrm{~N}$ tracer additions represented 51(8) \% and 208(31) \% of the ambient $\mathrm{NO}_{3}^{-}$and $\mathrm{NH}_{4}^{+}$pools, respectively. The predicted nutrient concentrations were an overestimate, in part due to the anomalously warm and nutrient-poor surface water mass that persisted in the region during our cruise (Bond et al., 2015; Seager et al., 2015). The potential biases that could result from the non-negligible tracer enrichments are discussed in section 4.6.3.

Analyses of PN concentration and isotopic ratios occurred at the University of California Santa Barbara Marine Science Institute. Measurement of ${ }^{15} \mathrm{~N}$ in the $\mathrm{NO}_{3}^{-}+\mathrm{NO}_{2}^{-}$pool occurred at the University of Connecticut in the Granger Lab by the denitrifier method (Sigman et al., 2001). Reference materials USGS32, USGS34 and USGS35 were analyzed in parallel to calibrate $\delta^{15} \mathrm{~N}$ values.

We calculated ${ }^{15} \mathrm{~N}$ uptake and nitrification following Dugdale and Goering (1967) and adjusted the $\mathrm{NH}_{4}^{+}$uptake and nitrification rates for isotope dilution following Kanda et al. (1987), by comparing the uptake estimates from the $6 \mathrm{~h}$ and $24 \mathrm{~h}$ incubations. Isotope dilution corrections increased $\mathrm{NH}_{4}^{+}$uptake by $30 \%$, and increased nitrification rates by 400 $\%$. Even with the revised, higher nitrification rate estimates, mixed layer nitrification rates were only $2 \%$ of $\mathrm{NO}_{3}^{-}$uptake.

We integrate the ${ }^{15} \mathrm{~N}$ uptake measurements using the depth at which each Niskin bottle was closed (rather than the light-equivalent depth) because the uptake rates are calculated with respect to the nutrient concentrations measured in the bottle. Since incubations were not performed at $100 \% \mathrm{I}_{o}$, we assumed the ${ }^{15} \mathrm{~N}$ uptake at $100 \% \mathrm{I}_{o}(0 \mathrm{~m}$ depth $)$ is equiva- 
lent to the uptake at $50 \% \mathrm{I}_{o}(\sim 2 \mathrm{~m}$ depth). We estimate the error of each daily incubation measurement to be $\pm 9 \%$ for $\mathrm{NO}_{3}^{-}$uptake, $\pm 23 \%$ for $\mathrm{NH}_{4}^{+}$uptake, and $\pm 15 \%$ for nitrification. These estimates are based on prior incubations of mixed layer water in Monterey Bay (unpublished results from coauthor Jason Smith). During this cruise, a single incubation was carried out at each depth.

\subsection{4 $\left[\mathrm{O}_{2}\right],[\mathrm{Ar}], \mathrm{O}_{2} / \mathrm{Ar}$, and ${ }^{17} \Delta$ analysis}

Throughout the cruise, we measured $\mathrm{O}_{2}$ and $\mathrm{Ar}$ concentrations $\left(\left[\mathrm{O}_{2}\right]\right.$ and $\left.[\mathrm{Ar}]\right), \mathrm{O}_{2} / \mathrm{Ar}$ mole ratios $\left(n\left(\mathrm{O}_{2}\right) / n(\mathrm{Ar})\right.$, abbreviated herein as $\left.\mathrm{O}_{2} / \mathrm{Ar}\right)$, and the triple oxygen isotopic composition of $\mathrm{O}_{2}\left(\delta^{17} \mathrm{O}, \delta^{18} \mathrm{O}\right.$, and $\left.{ }^{17} \Delta\right)$.

We used a field-deployable mass spectrometer (gas equilibration mass spectrometer, GEMS) to measure $\mathrm{O}_{2} / \mathrm{Ar}$ near-continuously from the underway system (2 $\mathrm{m} \mathrm{depth}$ ). We configured the instrument as described in Manning et al. (2016a), except that the getter chambers were removed and the mass spectrometer was a Pfeiffer PrismaPlus QMG200. The system is similar to the equilibrator inlet mass spectrometer developed by Cassar et al. (2009). For calibration, the system measured $\mathrm{O}_{2} / \mathrm{Ar}$ in air for $40 \mathrm{~min}$ after roughly $4 \mathrm{~h}$ of sampling the headspace of the equilibrator cartridge. We manually controlled the exact switching times to ensure that we obtained data while on station for the CTD casts whenever possible.

We analyzed 154 discrete samples from either the underway seawater system or from Niskin bottles for $\mathrm{O}_{2} / \mathrm{Ar}, \delta^{17} \mathrm{O}$, and $\delta^{18} \mathrm{O}$ with a Thermo Fisher Scientific MAT 253 isotope ratio mass spectrometer at the Woods Hole Oceanographic Institution. We followed the protocol of Barkan and Luz (2003) with some modifications (Stanley et al., 2010, 2015). We analyzed samples $4-7$ months after the evacuation of the flasks (3-5 months after sampling). The precision (mean standard deviation) of replicate field samples from the same Niskin bottle was 5.0 per meg for ${ }^{17} \Delta, 0.015 \%$ for $\delta^{17} \mathrm{O}, 0.031 \%$ for $\delta^{18} \mathrm{O}$, and $0.12 \%$ for $\Delta\left(\mathrm{O}_{2} / \mathrm{Ar}\right)$. For samples from the underway seawater line collected in rapid succession (within $8 \mathrm{~min}$ ), the precision was 6.7 per meg for ${ }^{17} \Delta, 0.023 \%$ for $\delta^{17} \mathrm{O}, 0.047 \%$ for $\delta^{18} \mathrm{O}$, and 0.38 $\%$ for $\Delta\left(\mathrm{O}_{2} / \mathrm{Ar}\right)$, and this lower precision is likely related to real environmental variability that is not captured in samples collected from the same Niskin bottle. See section 4.5.2 for more details on the triple oxygen isotope terminology.

On the day before the cruise departed, we flushed the underway line with bleach to remove biofilms that could cause high rates of respiratory consumption of $\mathrm{O}_{2}$ (Juranek et al., 2010). After the cruise, we evaluated the agreement between the different sampling and analytical methods. Using all discrete near-surface samples (underway and Niskin) the average difference between the discrete samples and the GEMS was -0.05(1.11)\%. Furthermore, the mean offset between the surface Niskin samples and the underway GEMS was 0.14(1.22)\%. We did not apply any correction to the GEMS data because these mean offsets were very 
small relative to the uncertainty in the offset.

We obtained measurements of $\left[\mathrm{O}_{2}\right]$ using a SBE 43 sensor on the CTD rosette and calibrated the sensor via Winkler titration (Carpenter, 1965). Using 25 water samples collected throughout the cruise and analyzed at sea, we calculated one calibration for all $\mathrm{O}_{2}$ data: $\left[O_{2}\right]_{\text {cal }}=1.0075\left[O_{2}\right]_{\text {meas }}+2.87\left(\mathrm{R}^{2}=0.9993\right)$ with $\left[O_{2}\right]_{\text {cal }}$ and $\left[O_{2}\right]_{\text {meas }}$ the calibrated

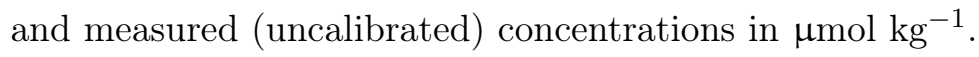

We also collected discrete samples for measurement of noble gas concentrations ( $\mathrm{He}, \mathrm{Ne}$, Ar, Kr, and Xe). Briefly, water samples were collected in copper tubes and sealed with a cold pressure welder; gas was subsequently extracted from the water and analyzed using a quadrupole mass spectrometer (Stanley et al., 2009a; Loose et al., 2016). In this paper we only use the $[\mathrm{Ar}]$ results, which had an estimated error (combined precision and accuracy) of $0.24 \%$ RSD (relative standard deviation). See Manning et al. (2016c) for the complete noble gas data.

\subsubsection{Sediment trap-based particle fluxes}

We deployed shallow particle interceptor traps (PITS) composed of three individual plastic collection tubes, each $1.4 \mathrm{~L}$ in volume (Knauer et al., 1979) at $50 \mathrm{~m}$ depth, connected to a surface drifter. We filled the traps with 0.2 micron filtered seawater prior to deployment and added two $10 \mathrm{~g} \mathrm{NaCl}$ pills to the base of each tube to form a dense brine to retain the particulate material. Deployments lasted $\sim 24 \mathrm{~h}$, beginning daily between dawn and noon. Once on deck, using 0.2 micron filtered seawater, we emptied each collection tube, rinsed three times, and made each sample up to $2.0 \mathrm{~L}$. Then we filtered $0.5 \mathrm{~L}$ from each $2.0 \mathrm{~L}$ sample and froze the filters for subsequent analysis for POC and PON content at the University of California Santa Barbara Marine Science Institute by online combustion. For five of the six deployments, we removed another $33 \mathrm{~mL}$ from each sample and then combined the three fractions and analyzed the samples by microscopy for quantitative taxonomic identification of zooplankton swimmers. We used this data to estimate the zooplankton volume and carbon content (Nozais et al., 2005). Estimates of the swimmer C flux from individual traps ranged from $8-145 \%$ of the total POC flux from the same trap.

\subsubsection{Nutrients and pigments}

We analyzed concentrations of phaeopigments and chlorophyll by filtering water samples onto glass fiber filters $(\mathrm{GF} / \mathrm{F})$, extracting pigments with acetone, and analyzing with a Turner fluorometer (Pennington and Chavez, 2000). We measured ammonium concentration on fresh unfiltered seawater samples immediately after collection using a fluorimetric method (Holmes et al., 1999), and for all other nutrients, we froze unfiltered samples aboard ship (Dore and Karl, 1996) for later analysis with an Alpkem 300 autoanalyzer (Sakamoto et al., 1990). Detection limits were 0.5, 0.5, and $0.01 \mu \mathrm{mol} \mathrm{kg}{ }^{-1}$ for $\mathrm{SiO}_{4}, \mathrm{NO}_{3}^{-}$and $\mathrm{NH}_{4}^{+}$ 
respectively, and error was $\sim 0.6 \%, 3 \%$, and $0.5 \%$ at maximum range for $\mathrm{SiO}_{4}, \mathrm{NO}_{3}^{-}$and $\mathrm{NH}_{4}^{+}$.

\subsubsection{Satellite data}

We used satellite-based sea surface temperature (SST) maps from MODIS Aqua and Terra (JPL/OBPG/RSMAS, 2006a,b) to assess the spatial structure of near-surface temperature within the bay. To determine whether the satellite data were contaminated by cloud cover, we examined true-color satellite images, which showed that 25-26 September and 30 September-3 October were nearly cloud-free within Monterey Bay whereas 27-29 September had significant clouds within the bay. We included SST pixels with a quality level of 2 or higher (level 5 is the highest quality), and eliminated the most questionable data (level 1). Many of the pixels masked at quality levels 2-4, predominantly in relatively small areas to the north and south of Monterey Bay near the coast, persisted in the same locations and shapes in multiple images separated by many hours or days. This indicates those temperature features were likely real oceanographic features (SST fronts and cold filaments) rather than clouds. Incorrect flagging of strong SST fronts as clouds is a known issue in the MODIS quality algorithms in coastal regions (Oram et al., 2008; Nieto et al., 2012). SST data within the main study area are not sensitive to the choice of quality level.

\subsection{Calculations}

\subsubsection{Calculation of NOP from $\mathrm{O}_{2} / \mathrm{Ar}$ mass balance}

We quantify the biologically-generated supersaturation (or undersaturation) of $\mathrm{O}_{2}$ with respect to Ar using the tracer $\Delta\left(\mathrm{O}_{2} / \mathrm{Ar}\right)$ :

$$
\Delta\left(O_{2} / A r\right)=\left[\frac{\left(O_{2} / A r\right)}{\left(O_{2} / A r\right)_{e q}}-1\right] .
$$

Here $\left(\mathrm{O}_{2} / \mathrm{Ar}\right)$ and $\left(\mathrm{O}_{2} / \mathrm{Ar}\right)_{e q}$ are the measured and equilibrium mole ratios, respectively, and $\Delta\left(\mathrm{O}_{2} / \mathrm{Ar}\right)$ is often expressed in percent by multiplying by $100 \% . \Delta \mathrm{O}_{2}$ is defined similarly, by removing Ar from equation 4.3. We calculate the gas saturation states using the solubility data of Hamme and Emerson (2004a) for Ar and Garcia and Gordon (1992) for $\mathrm{O}_{2}$. For the gas diffusivity, we use the data of Ferrell and Himmelblau (1967) for $\mathrm{O}_{2}$ and extrapolated values for Ar based on the data for other noble gases in Jähne et al. (1987a); Manning and Nicholson (2016).

Net $\mathrm{O}_{2}$ production (NOP) measures the net autotrophic production of $\mathrm{O}_{2}$ minus community respiratory consumption by autotrophs and heterotrophs. We define the biological 
$\mathrm{O}_{2},\left[\mathrm{O}_{2}\right]_{B}$ as

$$
\left[\mathrm{O}_{2}\right]_{B}=\Delta\left(\mathrm{O}_{2} / \mathrm{Ar}\right)\left[\mathrm{O}_{2}\right]_{e q} \frac{[\mathrm{Ar}]}{[\mathrm{Ar}]_{e q}}=\left[\mathrm{O}_{2}\right]-\left[\mathrm{O}_{2}\right]_{e q} \frac{[\mathrm{Ar}]}{[\mathrm{Ar}]_{e q}}
$$

We include the term $[\mathrm{Ar}] /[\mathrm{Ar}]_{e q}$, in contrast to other studies where it is often assumed that $[\mathrm{Ar}] /[\mathrm{Ar}]_{e q}=1$ if $[\mathrm{Ar}]$ is not directly measured (Hamme and Emerson, 2006; Stanley et al., 2010; Giesbrecht et al., 2012). Our discrete Ar samples indicate that $[\mathrm{Ar}] /[\mathrm{Ar}]_{e q}=$ 1.036(0.006) within the mixed layer and displayed no consistent gradients with depth or time (Manning et al., 2016c); we used this average value to calculate $\left[\mathrm{O}_{2}\right]_{B}$ throughout the cruise, which increases $\left[\mathrm{O}_{2}\right]_{B}$ by $3.6 \%$, compared to assuming $[\mathrm{Ar}] /[\mathrm{Ar}]_{e q}=1$.

We calculate the steady state mixed layer $\mathrm{NOP}$ as the rate of biological $\mathrm{O}_{2}$ production needed to balance the loss of biological $\mathrm{O}_{2}$ to gas exchange and vertical mixing (Cassar et al., 2011, 2014).

$$
N O P=k_{O_{2, w}}\left[O_{2}\right]_{B}+K_{z} \frac{\partial\left[O_{2}\right]_{B}}{\partial z}
$$

where $k_{O_{2, w}}$ is the time-weighted gas transfer velocity for $\mathrm{O}_{2}\left(\mathrm{~m} \mathrm{~d}^{-1}\right)$, calculated using the algorithm of Reuer et al. (2007) with the wind speed-based parameterization of Sweeney et al. (2007), and $K_{z}$ is the vertical diffusivity $\left(\mathrm{m}^{2} \mathrm{~d}^{-1}\right)$ from microstructure profiles. The term $k_{O_{2, w}}$ is calculated using winds measured at mooring M1. During the entire cruise $k_{O_{2, w}}=1.79(0.07) \mathrm{m} \mathrm{d}^{-1}$. We omit lateral advection from the mass balance because we did not observe consistent gradients in $\left[\mathrm{O}_{2}\right]_{B}$ in the mixed layer between our main sampling area (within $5 \mathrm{~km}$ of the mean cast location) and the water outside this area (Figures C-1-C-2). We calculate $\left[\mathrm{O}_{2}\right]_{B}$ over the mixed layer, which is shallower than the euphotic zone depth $\left(1 \% I_{o}\right)$, because we have continuous $\mathrm{O}_{2} / \mathrm{Ar}$ measurements at the surface only and because the subsurface physical fluxes of $\mathrm{O}_{2}$ are more difficult to quantify because they are due to mixing and transport, rather than air-sea exchange. Using the CTD $\left[\mathrm{O}_{2}\right]$ profiles every $6 \mathrm{~h}$ and/or the $\mathrm{O}_{2} / \mathrm{Ar}$ profiles measured more sporadically, we could not distinguish the small subsurface $\left[\mathrm{O}_{2}\right]_{B}$ fluxes from the large fluxes due to physical processes such as internal waves and lateral advection.

In this study, we used $K_{z}$ profiles obtained during the cruise to parameterize the vertical flux of $\left[\mathrm{O}_{2}\right]_{B}$ out of the mixed layer. In many other studies, $K_{z}$ is estimated based on past measurements, which can induce a factor of 10 uncertainty into the vertical $\mathrm{O}_{2}$ flux, and is sometimes the largest source of error in estimates of NOP (Hamme and Emerson, 2006; Giesbrecht et al., 2012; Jonsson et al., 2013; Cassar et al., 2014; Weeding and Trull, 2014). For each CTD cast that was immediately preceded by microstructure profiles, we calculated the vertical biological $\mathrm{O}_{2}$ gradient, $\partial\left[\mathrm{O}_{2}\right]_{B} / \partial z$ from a linear regression of the measured $\left[\mathrm{O}_{2}\right]_{B}$ profile from the base of the mixed layer to $10 \mathrm{~m}$ below the mixed layer (Figure 4-1b). We multiplied this vertical gradient by $K_{z}$ at the mixed layer depth, linearly interpolated to the same time as the CTD cast (Figure 4-1a). The average $\mathrm{K}_{z}$ at the base of the mixed layer was $1.6(2.4) \times 10^{-5} \mathrm{~m}^{2} \mathrm{~s}^{-1}$ and the range was $9.5 \times 10^{-7}-1.1 \times 10^{-4} \mathrm{~m}^{2} \mathrm{~s}^{-1}$. This result is 
similar to estimates by Haskell et al. (2016b) who determined $\mathrm{K}_{z}=1.5(0.6) \times 10^{-5} \mathrm{~m}^{2} \mathrm{~s}^{-1}$ at the base of the mixed layer during fall and winter in the Southern California Bight, using a wind speed-based parameterization of turbulence due to shear (Haskell et al., 2016a). The average vertical biological $\mathrm{O}_{2}$ flux was $-3.5(4.6) \mathrm{mmol}_{2} \mathrm{~m}^{-2} \mathrm{~d}^{-1}$, and the range was -19.0 to $-0.1 \mathrm{mmol} \mathrm{O}_{2} \mathrm{~m}^{-2} \mathrm{~d}^{-1}$. One cast yielded a much larger magnitude flux of $-95 \mathrm{mmol}$ $\mathrm{O}_{2} \mathrm{~m}^{-2} \mathrm{~d}^{-1}$; this cast was not included in the average $\mathrm{K}_{z}$ and $\mathrm{O}_{2}$ fluxes because it was an outlier. Due to the wide range in $\mathrm{O}_{2}$ fluxes estimated from individual profiles, and the lack of consistent changes with time, we applied the average vertical biological $\mathrm{O}_{2}$ flux to all casts. Vertical mixing increased the calculated NOP by less than $10 \%$, and constraining this flux reduces uncertainty in our NOP calculations.
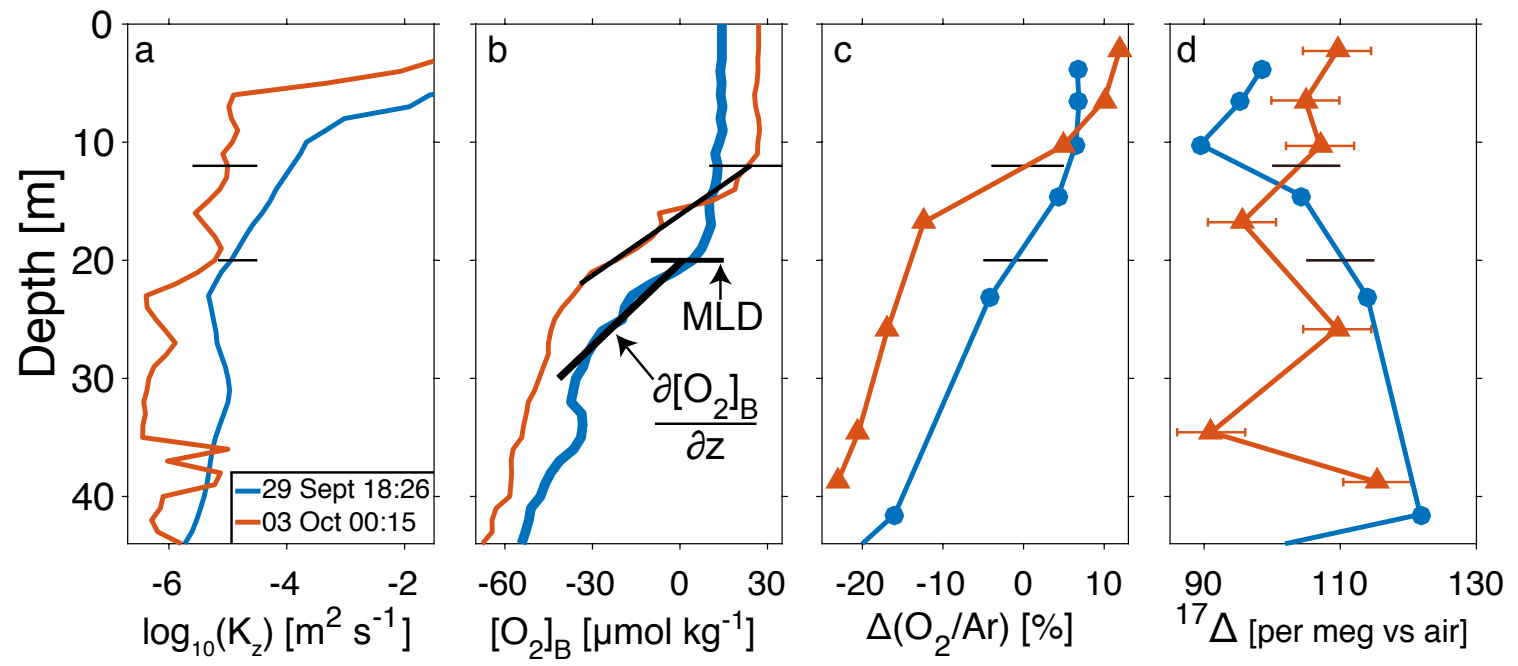

Figure 4-1: Representative profiles of (a) $\mathrm{K}_{z}$, (b) $\mathrm{O}_{2}$, (c) $\Delta\left(\mathrm{O}_{2} / \mathrm{Ar}\right.$ ), and (d) ${ }^{17} \Delta(\lambda=$ 0.5179). Black horizontal lines on (a-d) indicate mixed layer depth, and black slopes in (b) show the vertical gradient in $\mathrm{O}_{2}$. In (c), measurement error is smaller than filled symbols, and in (d), representative measurement error is shown on one profile with error bars.

For each cast, we define the $\mathrm{O}_{2}$-based mixed layer depth (MLD) as the first depth below $10 \mathrm{~m}$ where $\left[\mathrm{O}_{2}\right]$ is more than $1 \%$ different from the value at $10 \mathrm{~m}$, similar to Castro-Morales and Kaiser (2012). The MLD averaged 14(4) $\mathrm{m}$ and we used this average MLD of $14 \mathrm{~m}$ as the integration depth when calculating mixed layer productivity from the incubations (sections 4.6.2-4.6.3).

We convert NOP to NCP (from oxygen units to carbon units) using an $\mathrm{O}_{2}$ :C ratio (photosynthetic quotient) of 1.4 and 1.1 for $\mathrm{NO}_{3}^{-}$and $\mathrm{NH}_{4}^{+}$driven uptake, respectively (Laws, 1991). The $\mathrm{O}_{2}$ : C photosynthetic quotient was 1.33 in Phase 1 and 1.35 in Phase 2, based on the mixed layer $\mathrm{NO}_{3}^{-}$and $\mathrm{NH}_{4}^{+}$uptake incubations (section 4.6.3). 


\subsubsection{Calculation of GOP from ${ }^{17} \Delta$}

Using the triple isotopic composition of $\mathrm{O}_{2}$ we can quantify the rate of GOP. We characterize the isotopic composition of $\mathrm{O}_{2}$ using the tracers $\delta^{18} \mathrm{O}, \delta^{17} \mathrm{O}$, and ${ }^{17} \Delta$. We define $\delta^{18} \mathrm{O}=$ $X^{18} / X_{\text {air }}^{18}-1$. Here, $X^{18}=r\left({ }^{18} O /{ }^{16} O\right)$ is the measured ratio and $X_{\text {air }}^{18}=r\left({ }^{18} \mathrm{O} /{ }^{16} O\right)_{\text {air }}$ is the ratio of $\mathrm{O}_{2}$ in air, collected locally, which we used as the reference standard. The tracer $\delta^{17} \mathrm{O}$ is calculated analogously. The terms $\delta^{18} \mathrm{O}$ and $\delta^{17} \mathrm{O}$ are often expressed in \%o by multiplying by 1000 .

We define ${ }^{17} \Delta$ as

$$
{ }^{17} \Delta=\ln \left(\delta^{17} O+1\right)-\lambda \ln \left(\delta^{18} O+1\right) .
$$

Here, we use $\lambda=0.5179$ which is the empirically-determined ratio of the fractionation factors for respiratory $\mathrm{O}_{2}$ consumption in ${ }^{17} \mathrm{O}$ relative to ${ }^{18} \mathrm{O}$ (i.e. $\lambda={ }^{17} \epsilon /{ }^{18} \epsilon$ ) (Luz and Barkan, 2005). Using this definition, ${ }^{17} \Delta$ is not changed by respiration but is affected by photosynthesis, air-sea gas exchange, and mixing between water masses (Hendricks et al., 2005; Juranek and Quay, 2013; Nicholson et al., 2014). For dissolved $\mathrm{O}_{2}$ in water, ${ }^{17} \Delta$ is typically reported in per meg $\left(1\right.$ per meg $=0.001 \%$ ) due to the small range of values. $\mathrm{O}_{2}$ derived from air-sea equilibrium has ${ }^{17} \Delta \simeq 8$ per meg (Reuer et al., 2007; Stanley et al., 2010), and $\mathrm{O}_{2}$ that is purely biological in origin has ${ }^{17} \Delta \simeq 293$ per meg with $\lambda=0.5179$. Thus ${ }^{17} \Delta$ is a tracer of the proportion of $\mathrm{O}_{2}$ in a given water parcel that was generated from biological activities, relative to the amount added via air-sea exchange.

The most accurate method of calculating GOP from the triple oxygen isotopic composition of $\mathrm{O}_{2}$ has received much discussion in the literature. There is currently no single universally-accepted method of calculating GOP, and many authors have argued that the ideal choice of parameters and constants may depend on properties of the system (e.g., the microbial species of interest and isotopic composition of oxygen in water, which is the source of photosynthetic $\mathrm{O}_{2}$ ) (Luz and Barkan, 2000; Juranek and Quay, 2005; Reuer et al., 2007; Luz and Barkan, 2009; Stanley et al., 2010; Kaiser, 2011; Luz and Barkan, 2011; Nicholson et al., 2011; Prokopenko et al., 2011; Hamme et al., 2012). However, there is strong scientific consensus that calculations should be performed using the individual isotope ratios rather than the empirically defined term ${ }^{17} \Delta$ which is a combination of two isotope ratios (Kaiser, 2011; Luz and Barkan, 2011; Nicholson et al., 2011; Prokopenko et al., 2011). We include the raw data $\left(\delta^{17} \mathrm{O}, \delta^{18} \mathrm{O},{ }^{17} \Delta, \Delta\left(\mathrm{O}_{2} / \mathrm{Ar}\right),\left[\mathrm{O}_{2}\right]\right.$, salinity, temperature, depth, and wind speed) as a supplement to this paper (Data set 2) so that GOP can be re-calculated in the future using new formulas (Kaiser, 2011). We also provide the MATLAB code used to calculate GOP, to ensure that the calculations in this paper can be reproduced by others.

We use the following equation to calculate GOP (equation S8 from Prokopenko et al. 
(2011)):

$$
G O P=\frac{k\left[O_{2}\right]_{e q}\left[\frac{X^{17}-X_{e q}^{17}}{X^{17}}-\lambda \frac{X^{18}-X_{e q}^{18}}{X^{18}}\right]+h\left[O_{2}\right] \frac{\partial^{17} \Delta}{\partial t}}{\left[\frac{X_{p}^{17}-X^{17}}{X^{17}}-\lambda \frac{X_{p}^{18}-X^{18}}{X^{18}}\right]} .
$$

Here $h$ is the mixed layer depth, $k$ is the gas transfer velocity for $\mathrm{O}_{2}, X^{17}=r\left({ }^{17} \mathrm{O} /{ }^{16} \mathrm{O}\right)$ and the subscripts $p$ and $e q$ refer to $\mathrm{O}_{2}$ produced by photosynthesis and at air-sea equilibrium, respectively, and $\lambda=0.5179$ as in equation 5.7. Oxygen terms without a subscript $\left(\left[\mathrm{O}_{2}\right]\right.$, $X^{17}$, and ${ }^{17} \Delta$ ) are the measured mixed layer values. At steady state, i.e., if there is no change in ${ }^{17} \Delta$ with time, then $h\left[O_{2}\right] \partial^{17} \Delta / \partial t=0$, and equation 5.9 simplifies to equation 7 of Prokopenko et al. (2011).

We calculate $X_{e q}^{18}$ based on Benson and Krause (1984) and Benson and Krause (1980a), and $X_{e q}^{17}$ from Stanley et al. (2010) $\left({ }^{17} \Delta_{e q}=8\right.$ per meg, which is consistent with our daily analyses of equilibrated water at room temperature). We assume that the seawater (the substrate for photosynthetic $\mathrm{O}_{2}$ ) has the isotopic composition of VSMOW, as defined with respect to air in Barkan and Luz (2011), and that photosynthetic $\mathrm{O}_{2}$ has the isotopic composition of average phytoplankton defined in Luz and Barkan (2011). If we instead assume that the surface seawater in Monterey Bay has an isotopic composition of $\delta^{18} \mathrm{O}=$ -0.5761 and $\delta^{17} \mathrm{O}=-0.3092$ with respect to VSMOW, based on a global gridded data set of $\delta^{18} \mathrm{O}$ in seawater (LeGrande and Schmidt, 2006) and published relationships between $\delta^{17} \mathrm{O}$ and $\delta^{17} \mathrm{O}$ in seawater (Luz and Barkan, 2010), GOP is $\sim 11 \%$ higher. Since we did not collect water samples for measurement of the isotopic composition, and the global data set may be less accurate in coastal regions, we felt it was more appropriate to use the composition of VSMOW.

We omit the terms for kinetic isotopic fractionation during air-sea gas exchange in equation 5.9, which have been included in some other studies (Kaiser, 2011; Nicholson et al., 2011; Hamme et al., 2012; Nicholson et al., 2014). Including these terms decreases GOP by $1 \%$, which is well within the error of the method. In regions with higher wind speeds, this term will become more important. Also, we do not include a correction for vertical mixing/entrainment of $\mathrm{O}_{2}$ (Nicholson et al., 2012; Wurgaft et al., 2013; Nicholson et al., 2014) because we found that there was no consistent gradient in ${ }^{17} \Delta$ with depth below the mixed layer (Figure 4-1d).

Given that we did not observe a trend in ${ }^{17} \Delta$ with time, we calculated steady state GOP for each individual sample, then bin-averaged the samples using a $2 \mathrm{~h}$ window. This approach helped to ensure that times when sampling frequency was higher and replicates were analyzed were not over-weighted when calculating the average GOP.

We convert GOP to GPP (from oxygen units to carbon units) using $\mathrm{O}_{2}$ :C ratios of 1.33 in Phase 1 and 1.35 in Phase 2; the same ratios used for the conversion of NOP to NCP. 


\subsection{Results and discussion}

\subsubsection{Hydrographic and biogeochemical setting}

For this study, we consider all CTD/Niskin bottle data, all sediment trap data, and all underway data collected within a $5 \mathrm{~km}$ radius of the mean CTD profile location (Figure 4-2). The mean location is a central point where the average distance to all 23 CTD casts is minimized. The $5 \mathrm{~km}$ radius is the minimum distance that included all CTD cast locations. If we had included all underway measurements (Figure C-1) in our productivity estimates from $\mathrm{O}_{2}$, this could have generated more biases between the incubation/trap and $\mathrm{O}_{2}$ measurements due to the significant spatial variability in physical properties and productivity within Monterey Bay and contiguous waters that is recorded in the underway record but not the other methods (Pennington and Chavez, 2000; Ryan et al., 2009). Throughout the paper, all results are expressed as mean $( \pm$ standard deviation $)$ unless otherwise specified.
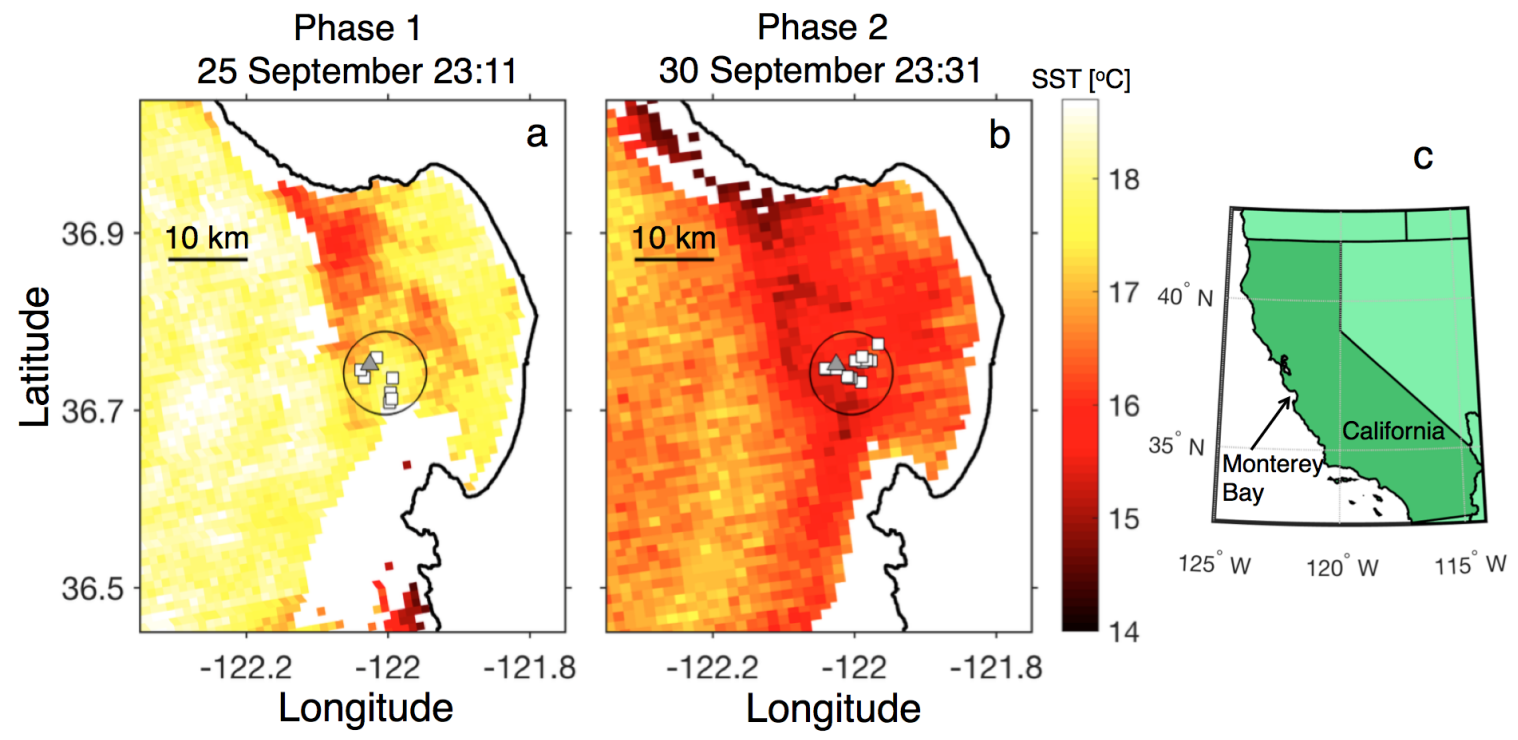

Figure 4-2: Map of study site showing MODIS sea surface temperature measured (a) 25 September 23:11 (representative of Phase 1) and (b) 30 September 23:31 (during Phase 2). White areas over water indicate no satellite SST data was available. White squares show locations of CTD casts in Phase 1 (a) and Phase 2 (b). Grey triangles show location of mooring M1. The black circle shows the region within $5 \mathrm{~km}$ of the mean profile. (c) Map of California, showing location of Monterey Bay.

We separate the cruise into two phases, based on the surface water characteristics (Figures 4-2-4-4). Phase 1 is a relatively stable period beginning with the first CTD cast on 27 September 15:00 and ending 29 September 09:35. Phase 2 is a more dynamic period beginning 29 September 09:35 and lasting through the end of the cruise, during which we sampled multiple water masses at the surface, including a colder, recently upwelled filament, as well as a warmer water mass in which the the water was biogeochemically similar to Phase 
1. During Phase 1, temperature was high $\left(16.45(0.18){ }^{\circ} \mathrm{C}\right), \Delta\left(\mathrm{O}_{2} / \mathrm{Ar}\right)$ was high $(9.8(1.8)$ $\%$ ), and macronutrient concentrations were low $\left(\left[\mathrm{NO}_{3}^{-}\right] \leqslant 0.2 \mu \mathrm{mol} \mathrm{kg}{ }^{-1}\right.$ for all seven casts and $\left[\mathrm{SiO}_{4}\right] \leqslant 1.0 \mu \mathrm{mol} \mathrm{\textrm {kg } ^ { - 1 }}$ for six out of seven casts) in the upper $4 \mathrm{~m}$. During Phase 2 , on average, the surface water in Phase 2 had lower temperature $\left(15.88(0.40){ }^{\circ} \mathrm{C}\right)$, lower $\Delta\left(\mathrm{O}_{2} / \mathrm{Ar}\right)(6.7(3.2) \%)$, and higher nutrient concentrations $\left(\left[\mathrm{NO}_{3}^{-}\right]=0.5(0.5) \mu \mathrm{mol} \mathrm{kg}{ }^{-1}\right.$, maximum $1.7 \mu \mathrm{mol} \mathrm{kg}{ }^{-1}$ and $\left[\mathrm{SiO}_{4}\right]=1.7(0.8) \mu \mathrm{mol} \mathrm{kg}{ }^{-1}$, maximum $3.0 \mu \mathrm{mol} \mathrm{kg}{ }^{-1}$ ).

We determined the start time for Phase 2 based on the ship's temperature record; Phase 2 began on 29 September when the sea surface temperature first dropped to $15.91{ }^{\circ} \mathrm{C}$, three standard deviations below the mean temperature observed in Phase 1. During the cast on 29 September 06:00, the mixed layer conditions were consistent with the other Phase 1 casts, and beginning with the cast on 29 September 12:00, the mixed layer conditions were more variable. Thus, Phase 2 began some time between these two casts. The exact start time for Phase 2 does not affect our conclusions. At the beginning of Phase 2 (between the morning of 29 September and 30 September $)$ there was a transition period when cold $\left(\sim 15^{\circ} \mathrm{C}\right)$ water with $\Delta\left(\mathrm{O}_{2} / \mathrm{Ar}\right) \simeq 0 \%$ persisted within the study area for several hours. During the rest of the cruise, the water was often colder and had lower $\mathrm{O}_{2}$ concentrations than in Phase 1 , but not as extreme as the conditions during the transition period. This observation suggests that the transition period water was advected out of the study area after the morning of 30 September.

Satellite-based sea surface temperature (SST) measurements supported our shipboard observations of changes in surface water properties in Monterey Bay during the cruise, as well as the presence of strong temperature fronts in Monterey Bay and contiguous waters. On the night of 25 September, surface waters were predominantly warm (Figure 4-2a). There was a patch of colder water at the northwestern edge of the bay, likely a filament of recently upwelled water, but it was outside our main sampling area (Figure 4-2a). Clouds over Monterey Bay obscured all SST images on the nights of 26-28 September. By nighttime on 30 September, SST was substantially colder throughout the bay. Our study area overlapped with the coldest water and somewhat warmer water was present to the east and west (Figure $4-2 b)$.

The above observations are suggestive of lateral advection of recently upwelled water into the study area during Phase 2. The transition between the two phases coincided with the highest total wind speeds and highest southward (upwelling-favorable) wind speeds observed during the cruise (Manning et al., 2016c). Our data suggest that during Phase 2, we sampled multiple water masses including a colder water mass with lower $\mathrm{O}_{2}$ and higher nutrients, consistent with recently upwelled water (Pennington and Chavez, 2000; Ryan et al., 2009; Johnson, 2010), a warmer water mass that had biogeochemical characteristics more similar to Phase 1, and also mixtures of these two water masses. Furthermore, there were often significant difference between the underway and M1 surface measurements of temperature, 


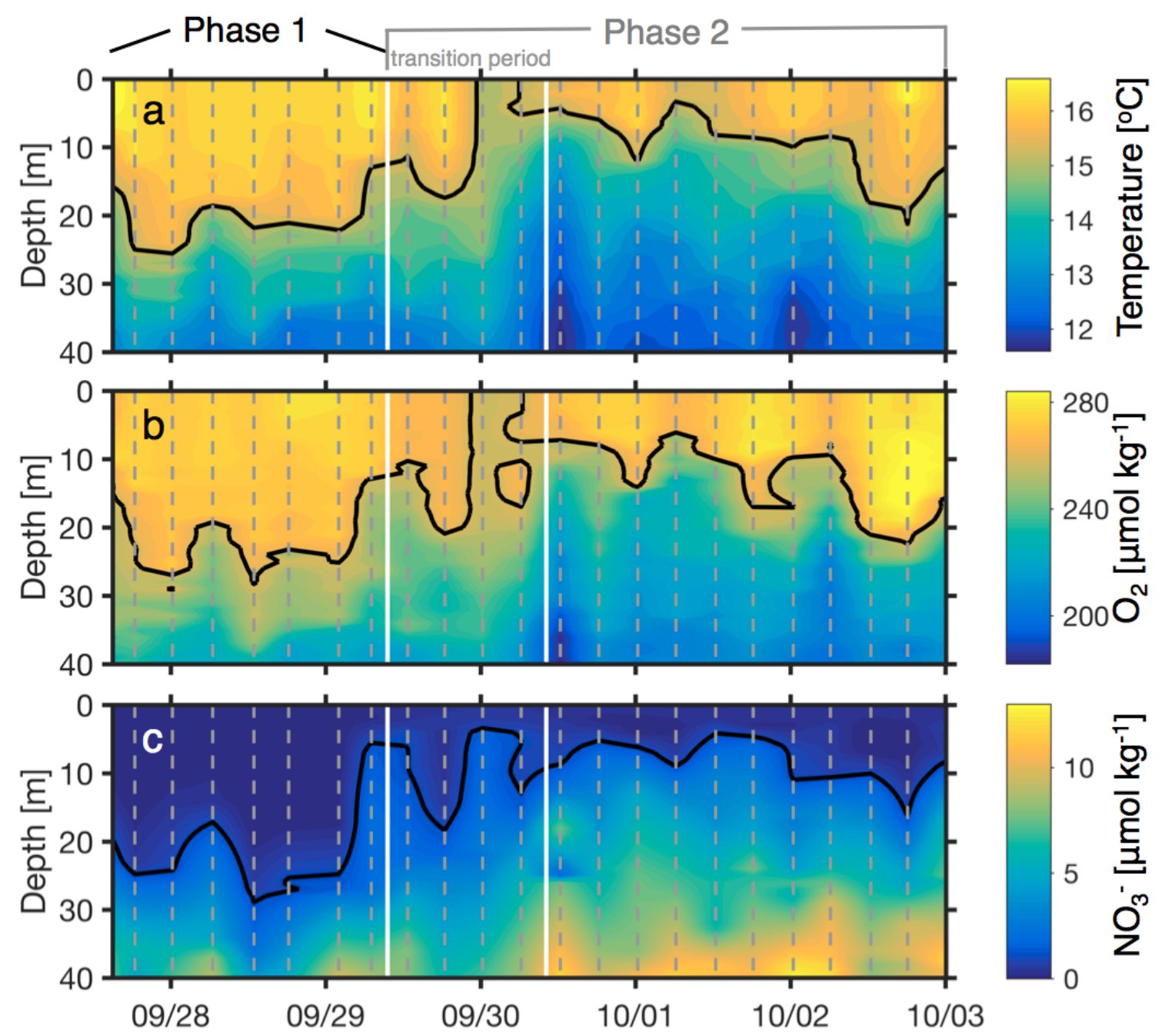

Figure 4-3: Measured profiles of (a) temperature, (b) $\left[\mathrm{O}_{2}\right]$, and (c) $\left[\mathrm{NO}_{3}^{-}\right]$during the cruise. The black contour lines are at (a) $15.9^{\circ} \mathrm{C}$, (b) $260 \mu \mathrm{mol} \mathrm{kg}-1$, and (c) $1.2 \mu \mathrm{mol} \mathrm{kg}{ }^{-1}$, and are selected to emphasize the colder water with lower $\mathrm{O}_{2}$ and higher $\mathrm{NO}_{3}^{-}$that entered the euphotic zone during Phase 2. The vertical grey dashed lines show the timing of CTD casts and the vertical white lines are at 29 September 09:35 and 30 September 10:10, the transition period at the start of Phase 2 when the coldest, lowest $\mathrm{O}_{2}$ surface water passed through the site. 

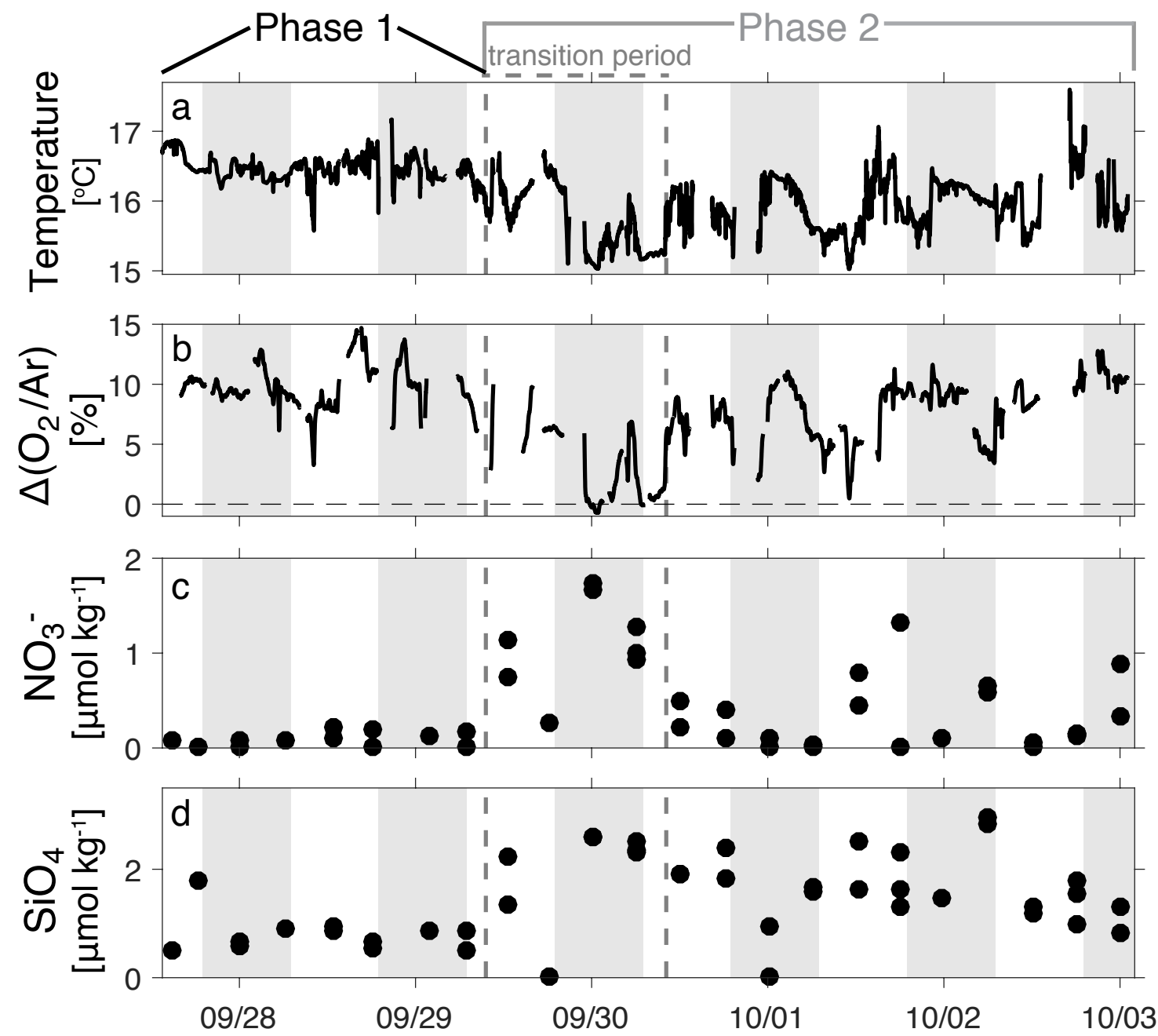

Figure 4-4: Near-surface ( $<4 \mathrm{~m}$ depth) measurements of (a) temperature from the ship's underway system, (b) $\Delta\left(\mathrm{O}_{2} / \mathrm{Ar}\right)$ from the underway mass spectrometer, (c) $\left[\mathrm{NO}_{3}^{-}\right]$from discrete Niskin samples, and $(\mathrm{d})\left[\mathrm{SiO}_{4}\right]$ from discrete Niskin samples. The vertical grey dashed lines indicate the transition period at the start of Phase 2 when the coldest, lowest $\mathrm{O}_{2}$ water passed through the site. The shaded grey bars indicate local nighttime (sunset to sunrise). See Figures C-1-C-2 for the spatial distribution of $\Delta\left(\mathrm{O}_{2} / \mathrm{Ar}\right)$ and temperature. 
$\left[\mathrm{NO}_{3}^{-}\right]$, and $\left[\mathrm{O}_{2}\right]$, especially during Phase 2, demonstrating that there was small-scale variability in these parameters within Monterey Bay (Figure 4-4 for underway data, mooring data not shown). In this paper we quantify differences in productivity between the two phases, and the impact of these different water masses on productivity estimates in Phase 2.

\subsection{2 ${ }^{14} \mathrm{C}$ incubations}

Here we focus on the incubation results integrated to the mean mixed layer depth for the cruise (14 m, section 4.6.4). This integration depth is most appropriate for comparison with the $\mathrm{O}_{2}$-based productivity estimates (section 4.6.4) (Hendricks et al., 2004; Hamme et al., 2012; Juranek and Quay, 2013). Euphotic zone ${ }^{14} \mathrm{C}-\mathrm{PP}$ (to $1 \% I_{o}$ ) was on average $11 \%$ higher than mixed layer ${ }^{14} \mathrm{C}-\mathrm{PP}$. In the supplementary information, we include all incubation results $\left(0.1-100 \% \mathrm{I}_{o}\right)$. We report all results for all methods as mean( \pm standard deviation $)$ except when noted otherwise.

Because water for the incubations on 29 September was collected at 06:00, when the euphotic zone was warm, had high $\mathrm{O}_{2}$, and low nutrients, we consider these incubations to be representative of Phase 1. Mixed layer ${ }^{14} \mathrm{C}-\mathrm{PP}$ was $90(22) \mathrm{mmol} \mathrm{C} \mathrm{m}{ }^{-2} \mathrm{~d}^{-1}$ in Phase 1 and $142(12) \mathrm{mmol} \mathrm{C} \mathrm{m}^{-2} \mathrm{~d}^{-1}$ in Phase 2, an increase of $57 \%$ (Figure 4-5c). We also note that the first incubation, which was initiated $3 \mathrm{~h}$ before sunset, is potentially biased somewhat higher (by $<30 \%$ ) than the other incubations which were initiated at dawn (Pennington et al., 2015).

\subsection{3 ${ }^{15} \mathrm{~N}$ incubations}

During this cruise, the euphotic zone was anomalously warm and low in nutrients compared to prior years, and tracer concentrations were significant relative to ambient conditions (section 4.4.3). The ${ }^{15} \mathrm{~N}$ tracer addition to bottles with low ambient DIN concentrations may have perturbed nutrient cycling within the incubation flasks and potentially caused our incubations to overestimate rates of $\mathrm{N}$ uptake and transformations, especially for $\mathrm{NH}_{4}^{+}$. This is a common issue for ${ }^{15} \mathrm{~N}$ incubations in N-limited marine systems, and with $\mathrm{NH}_{4}^{+}$ incubations in general (Goldman et al., 1981; Allen et al., 1996; Ward, 2005).

Despite these potential caveats, our ${ }^{15} \mathrm{~N}$ incubation results are generally consistent with the other methods (section 4.7). In Phase $1, \mathrm{NO}_{3}^{-}$uptake was lower and $\mathrm{NH}_{4}^{+}$uptake was similar, compared to Phase 2 (Figure 4-5a-b). Within the mixed layer, $\mathrm{NO}_{3}^{-}$uptake was $4.9(1.1) \mathrm{mmol} \mathrm{N} \mathrm{m}^{-2} \mathrm{~d}^{-1}$ in Phase 1 and 8.6(1.3) $\mathrm{mmol} \mathrm{N} \mathrm{m}^{-2} \mathrm{~d}^{-1}$ in Phase 2, an increase of $76 \%$ (Table 4-1). Mixed layer $\mathrm{NH}_{4}^{+}$uptake was $1.5(0.6)$ mmol N m${ }^{-2} \mathrm{~d}^{-1}$ in Phase 1 and 1.6(0.4) mmol $\mathrm{N} \mathrm{m}^{-2} \mathrm{~d}^{-1}$ in Phase 2. Nitrification rates were $2 \%$ of $\mathrm{NO}_{3}^{-}$uptake rates in Phase 1 and Phase 2. If we assume that $\mathrm{NH}_{4}^{+}$and $\mathrm{NO}_{3}^{-}$are the only $\mathrm{N}$ sources taken up by phytoplankton, $\mathrm{N}$ uptake increased $59 \%$ between Phase 1 and Phase 2 . 
Table 4-1: Mixed layer productivity and nitrification results $\left(\mathrm{mmol} \mathrm{m} \mathrm{m}^{-2} \mathrm{~d}^{-1}\right)^{a}$

\begin{tabular}{|c|c|c|c|c|c|c|}
\hline \multirow[b]{2}{*}{ Method } & \multicolumn{3}{|c|}{ Phase 1} & \multicolumn{3}{|c|}{ Phase 2} \\
\hline & $\mathrm{C}$ & $\mathrm{N}$ & $\mathrm{O}_{2}$ & $\mathrm{C}$ & $\mathrm{N}$ & $\mathrm{O}_{2}$ \\
\hline${ }^{14} \mathrm{C}-\mathrm{PP}$ & $90(22)$ & $11.8(2.9)$ & $120(29)$ & $142(12)$ & $11.8(1.0)$ & 192(16) \\
\hline${ }^{15} \mathrm{NO}_{3}^{-}$uptake & $37(8)$ & $4.9(1.1)$ & $49(11)$ & $65(10)$ & $8.6(1.3)$ & $88(14)$ \\
\hline${ }^{15} \mathrm{NH}_{4}^{+}$uptake & $11(4)$ & $1.5(0.6)$ & $15(6)$ & $12(3)$ & $1.6(0.4)$ & $16(4)$ \\
\hline Nitrification $^{b}$ & $0.8(0.7)$ & $0.10(0.09)$ & $-2.0(1.8)$ & $1.4(1.3)$ & $0.17(0.16)$ & $-0.34(0.32)$ \\
\hline $\mathrm{O}_{2} / \mathrm{Ar}-\mathrm{NCP}$ & $36(10)$ & $4.7(1.4)$ & $48(14)$ & $30(12)$ & $4.0(1.6)$ & $41(16)$ \\
\hline${ }^{17} \Delta-\mathrm{GPP}$ & $258(28)$ & $34(4)$ & $343(37)$ & $255(42)$ & $33.5(5.5)$ & $344(57)$ \\
\hline Particle flux ${ }^{c}$ & $21(19)$ & $2.7(2.5)$ & $28(26)$ & $21(19)$ & $2.7(2.5)$ & $28(26)$ \\
\hline
\end{tabular}

${ }^{a}$ Values are converted between $\mathrm{C}, \mathrm{N}$, and $\mathrm{O}_{2}$ using a C:N molar ratio of $7.6: 1$, based on the sediment trap POC:PON ratio (section 4.6.6), and a $\mathrm{C}: \mathrm{O}_{2}$ ratio of 1.33 in Phase 1 and 1.35 in Phase 2 based on the ratio of $\mathrm{NO}_{3}^{-}: \mathrm{NH}_{4}^{+}$uptake (section 4.6.4). Positive $\mathrm{C}$ and $\mathrm{N}$ values indicate uptake and positive $\mathrm{O}_{2}$ values indicate production. Values in bold are the units of the original measurement.

${ }^{b}$ Nitrification rates are converted from mol N:mol C using a ratio of 1:8.3 (Dore and Karl, 1996) and from mol $\mathrm{N}: m o l \mathrm{O}_{2}$ using a ratio of $1: 2$ based on the reaction chemistry $\left(\mathrm{NH}_{4}^{+}\right.$ $\left.+2 \mathrm{O}_{2} \rightarrow \mathrm{NO}_{3}^{-}+\mathrm{H}_{2} \mathrm{O}+2 \mathrm{H}^{+}\right)$.

${ }^{c}$ Measured at $50 \mathrm{~m}$ depth; all other methods integrated to the average mixed layer depth $(\sim 14 \mathrm{~m})$.

We converted the $\mathrm{N}$ uptake to $\mathrm{C}$ uptake based on sediment trap POC and PON measurements, which recorded a C:N molar ratio of 7.6(0.3) throughout the cruise, somewhat higher than the canonical Redfield ratio of 6.6 (Redfield et al., 1963). The ${ }^{15} \mathrm{~N}$ incubation results agree with our observations of higher euphotic zone $\mathrm{NO}_{3}^{-}$concentrations in Phase 2 compared to Phase 1 , which stimulated $\mathrm{NO}_{3}^{-}$uptake and primary production, and decreased $\mathrm{NH}_{4}^{+}$uptake and nitrification.

\subsubsection{NCP and NOP from $\mathrm{O}_{2} / \mathrm{Ar}$}

Throughout Phase $1, \Delta\left(\mathrm{O}_{2} / \mathrm{Ar}\right)=9.8(1.8) \%$ and showed no consistent diurnal cycle, nor any trend with time (Figure 4-4). Assuming steady state in Phase 1, NOP was 47(14) mmol $\mathrm{O}_{2} \mathrm{~m}^{-2} \mathrm{~d}^{-1}$. The uncertainty is calculated by propagating uncertainty in $\left[\mathrm{O}_{2}\right]_{B}(18$ $\%), k_{w, O 2}(10 \%$, based on the agreement between Sweeney et al. (2007) and other recent wind speed-based parameterizations (Ho et al., 2006; Wanninkhof et al., 2009; Wanninkhof, 2014)), and the vertical flux of $\left[\mathrm{O}_{2}\right]_{B}\left(4.6 \mathrm{mmol} \mathrm{O}_{2} \mathrm{~m}^{-2} \mathrm{~d}^{-1}\right.$, Table 4-1). Although NOP follows a diurnal cycle (Johnson, 2010; Wilson et al., 2015), spatial variability in $\mathrm{O}_{2} / \mathrm{Ar}$, lateral transport, and variable rates of vertical mixing may have confounded the observation of a diurnal cycle in $\mathrm{O}_{2} / \mathrm{Ar}$ during this time-series. 


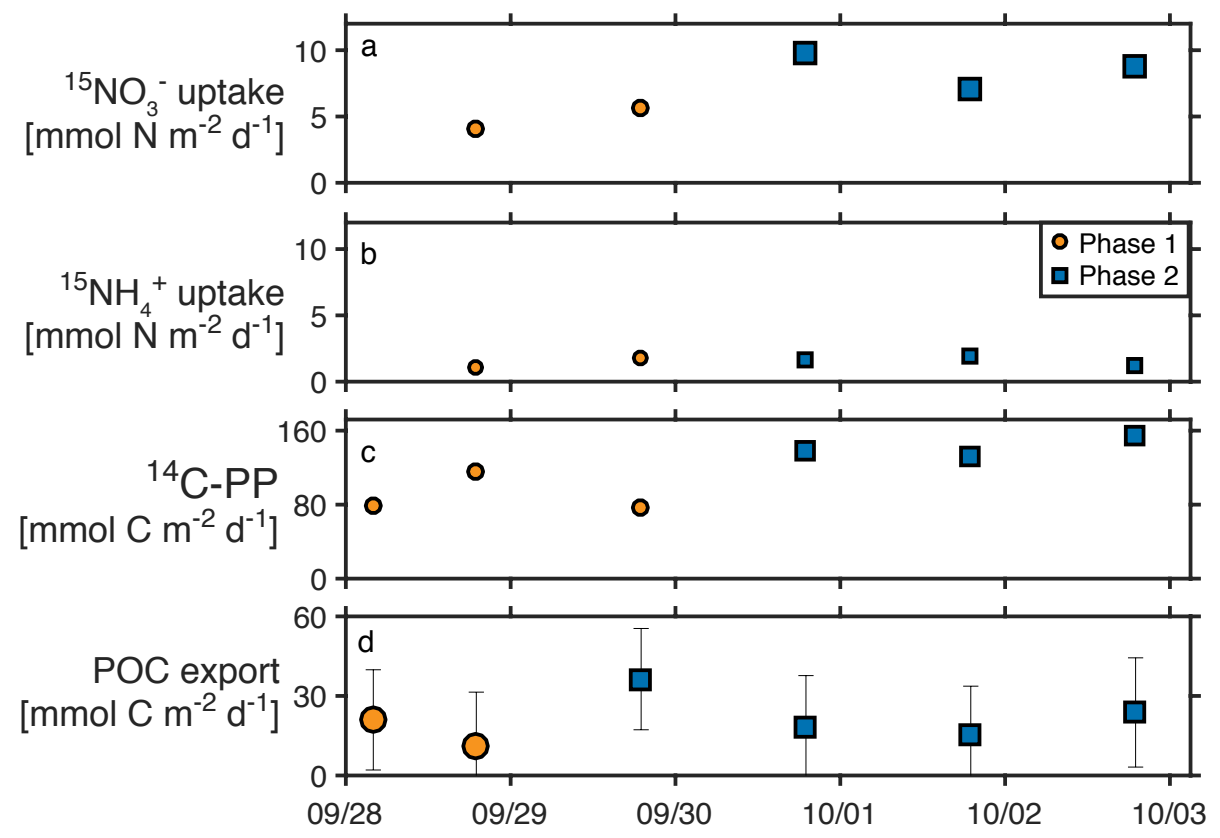

Figure 4-5: Results of depth-integrated incubations measuring (a) ${ }^{15} \mathrm{NO}_{3}^{-}$uptake, (b) ${ }^{15} \mathrm{NH}_{4}^{+}$uptake, and (c) ${ }^{14} \mathrm{C}-\mathrm{PP}$, as well as (d) sediment trap-based POC flux to $50 \mathrm{~m}$. Orange circles indicate data representative of Phase 1, and blue squares show data representative of Phase 2. For $(\mathrm{a}-\mathrm{c})$ the size of the symbols approximates the measurement error.

In Phase 2, we measured a larger range of values for surface $\Delta\left(\mathrm{O}_{2} / \mathrm{Ar}\right)$, with a minimum of $-0.7 \%$ between midnight and 09:30 on 30 September, and a maximum of $12.8 \%$ near sunset on 2 October. The average $\Delta\left(\mathrm{O}_{2} / \mathrm{Ar}\right)$ in Phase 2 was $6.7(3.2) \%$ beginning at 29 September 09:35 (when the colder water began to enter the study area) or 7.9(2.4) \% beginning on 30 September 10:10 (immediately following the transition period, when the coldest water with lowest $\mathrm{O}_{2}$ concentrations was present in the study area). Steady state NOP beginning on 30 September 10:10 was 41(16) $\mathrm{mmol} \mathrm{O}_{2} \mathrm{~m}^{-2} \mathrm{~d}^{-1}$, which is $14 \%$ lower than NOP in Phase 1.

We argue that it is appropriate to exclude the measurements during the transition period between 29 September 09:35 and 30 September 10:10 when calculating NOP because the recently-upwelled water sampled at the surface during this period was clearly far removed from steady state. For example, if NOP remained constant throughout the cruise at the rate calculated during Phase 1 and advective $\mathrm{O}_{2}$ fluxes were negligible, the steady state $\Delta\left(\mathrm{O}_{2} / \mathrm{Ar}\right)$ in Phase 2 would be $10 \%$ (Figure C-3), rather than $0 \%$, the value observed at 30 September 00:00.

If we assume that NCP increased $57 \%$ in Phase 2 (equivalent to the increase in ${ }^{14} \mathrm{C}-\mathrm{PP}$ ), we would expect $\Delta\left(\mathrm{O}_{2} / \mathrm{Ar}\right)$ to increase from 0 to $5 \%$ between 30 September 00:00 and 3 October 00:00, or to increase from 6 to $9 \%$ between 30 September 10:10 and 3 October 00:00 (Figure C-3). The steady state $\Delta\left(\mathrm{O}_{2} / \mathrm{Ar}\right)$ is $15 \%$ and the system would take $\sim 30$ 
days to reach steady state. Even if NOP remained constant in Phase 2, we would expect $\Delta\left(\mathrm{O}_{2} / \mathrm{Ar}\right)$ to increase by $1-3 \%$ by the end of the cruise (Figure C-3). The wide range of measured values of $\Delta\left(\mathrm{O}_{2} / \mathrm{Ar}\right)$ and the lack of a consistent increase in $\Delta\left(\mathrm{O}_{2} / \mathrm{Ar}\right)$ during Phase 2 suggests that we were indeed sampling multiple biogeochemically distinct water masses during Phase 2.

Due to the spatial variability in $\mathrm{O}_{2}$, we are not able to determine the rate of change in $\Delta\left(\mathrm{O}_{2} / \mathrm{Ar}\right)$ with time, which is needed for a non-steady state calculation of NOP (Hamme et al., 2012; Tortell et al., 2014; Wilson et al., 2015; Palevsky et al., 2016a). For example, we can calculate a linear regression of $\Delta\left(\mathrm{O}_{2} / \mathrm{Ar}\right)$ versus time over any $48 \mathrm{~h}$ period in Phase 2 (beginning 30 September 10:10 or later), with the $48 \mathrm{~h}$ period chosen to prevent diurnal cycling in $\mathrm{O}_{2}$ from biasing the slope (Hamme et al., 2012). For the $48 \mathrm{~h}$ regressions, the slope ranges from $0.5-1.4 \% \mathrm{~d}^{-1}$ (Figure C-4) and the variability in the slope caused by small shifts in the start and end time indicates that we would have low confidence in any non-steady state term.

Potentially, we could calculate the non-steady state $\mathrm{O}_{2}$ flux using only measurements in the recently upwelled water mass, which likely exhibited the biggest change in productivity and $\Delta\left(\mathrm{O}_{2} / \mathrm{Ar}\right)$. However, we were unable to separate the time-series in Phase 2 into different water masses with different histories (Figure C-5). Although the water sampled in Phase 2 ranged from $15.0-17.6{ }^{\circ} \mathrm{C}$, the salinity range was narrow (most measurements between 33.35-33.40 PSS) and a wide range of $\mathrm{O}_{2}$ saturation anomalies were observed across the range of salinity values (Figure C-5). Temperature in the mixed layer will be strongly affected by surface heat fluxes and cannot be used as a unique water mass tracer (Price et al., 1986; Cayan, 1992; Large et al., 1994). Our high-frequency $\mathrm{O}_{2} /$ Ar data enabled us to detect multiple, biogeochemically distinct water masses within our study area during Phase 2 , but our estimated NOP during Phase 2 is likely an underestimate as we were unable to calculate the non-steady state $\mathrm{O}_{2}$ flux.

If we had continuously sampled within a single recently upwelled filament during Phase 2 , the changes in $\mathrm{O}_{2}$ would become apparent by the end of the time-series and it would be possible to calculate the non-steady state $\mathrm{O}_{2}$ flux (Figure C-3). Alternatively, if we had an additional tracer that could be used to separate the time-series into different water masses (e.g., $\mathrm{NO}_{3}{ }^{-}$or salinity), this would have facilitated our estimation of the non-steady state $\mathrm{O}_{2}$ fluxes inside and outside of the filament of recently upwelled water. Finally, if the time-series had continued for longer during Phase 2 (i.e. 5-10 days instead of 3 days) and productivity within the recently upwelled filament had continued at a higher rate compared to outside of the filament, the magnitude of the total increase in $\Delta\left(\mathrm{O}_{2} / \mathrm{Ar}\right)$ (the non-steady state $\mathrm{O}_{2}$ flux) within the filament during the observation period would have been larger and may have been easier to quantify, despite the fact that the ship was transiting through different water masses. 

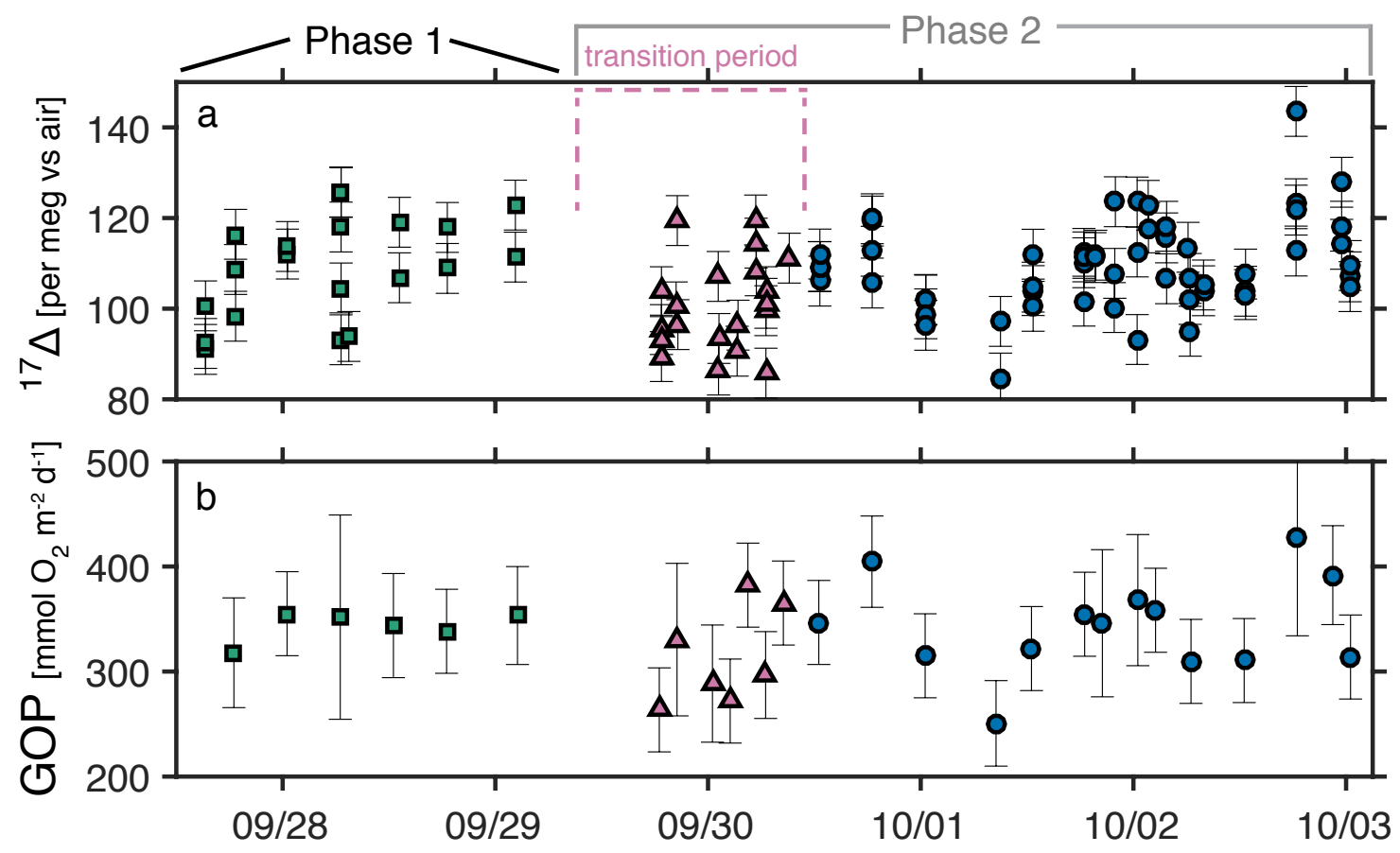

Figure 4-6: (a) Mixed layer ${ }^{17} \Delta(\lambda=0.5179)$, showing all samples, and (b) steady state GOP, calculated with 2-h bin averaging. The green squares are during Phase 1 and the pink triangles and blue circles are during Phase 2. In (b) the blue circles were included in the average GOP calculation, and the pink triangles are samples collected during the transition period at the start of Phase 2 when we sampled the coldest, lowest $\mathrm{O}_{2}$ surface water; we excluded these samples from the Phase 2 average.

\subsubsection{GPP and GOP from ${ }^{17} \Delta$}

Using the steady state equation, GOP was 343(37) $\mathrm{mmol} \mathrm{O}_{2} \mathrm{~m}^{-2} \mathrm{~d}^{-1}$ in Phase 1 and 334(58) mmol $\mathrm{O}_{2} \mathrm{~m}^{-2} \mathrm{~d}^{-1}$ in Phase 2 beginning at 29 September 09:35, or 344(57) $\mathrm{mmol} \mathrm{O}_{2} \mathrm{~m}^{-2}$ $\mathrm{d}^{-1}$ in Phase 2 beginning at 30 September 10:10 (following the transition period, as done for $\mathrm{O}_{2} / \mathrm{Ar}$, Figure 4-4). The uncertainty in GOP is calculated by propagating the uncertainty in $k\left(10 \%\right.$, section 4.6.4) and the standard deviation of $\mathrm{GOP} / k\left[O_{2}\right]_{e q}$ (equation 5.9), after averaging the samples into $2 \mathrm{~h}$ bins.

Our steady state calculation suggests that GOP was similar in Phase 1 and Phase 2, however, as discussed above for $\mathrm{O}_{2} / \mathrm{Ar}$ (section 4.6.4), $\mathrm{O}_{2}$ may not have been in steady state during Phase 2, and it is likely that we sampled different water masses with unique biogeochemical characteristics during Phase 2. We did not observe a consistent increase in ${ }^{17} \Delta$ during Phase 2 and therefore were unable to calculate a non-steady state GOP. We calculated that to generate a $57 \%$ increase in GOP in Phase 2 relative to Phase 1 (equivalent to the increase in $\left.{ }^{14} \mathrm{C}-\mathrm{PP}\right)$, we can add a non-steady state term, $\partial^{17} \Delta / \partial t=7$ per meg d ${ }^{-1}$ in equation 5.9. Thus a $\sim 20$ per meg increase in GOP between 30 September 10:10 and 3 October 01:00 would be consistent with the observed increase in ${ }^{14} \mathrm{C}-\mathrm{PP}$. Although samples 
from Phase 2 did not show a steady increase in ${ }^{17} \Delta$ throughout Phase $2,{ }^{17} \Delta$ was $109(2)$ per meg on 30 September 12:30, and 118(12) per meg between 2 October 18:30 to 3 October 00:30, an increase of 9 per meg. A small change in ${ }^{17} \Delta$ measured for a short period of time may be indistinguishable from other sources of variability/error, such as the precision of replicate samples (5-7 per meg), the variability within the mixed layer (often greater than $5-7$ per meg), diurnal changes in ${ }^{17} \Delta$ (Sarma et al., 2010) and spatial variability in GOP. If the cruise had extended for longer in Phase 2, with GOP maintained at a higher, constant rate, the change in ${ }^{17} \Delta$ would become more apparent with time. When $\mathrm{O}_{2}$ is at steady state, a $57 \%$ difference in GOP is easily distinguishable with the triple oxygen isotope method; for the MLD and wind speed at our study site, a steady state mixed layer GOP $=320 \mathrm{mmol}$ $\mathrm{m}^{-2} \mathrm{~d}^{-1}$ yields ${ }^{17} \Delta \sim 110$ per meg and GOP $=500 \mathrm{mmol} \mathrm{m}{ }^{-2} \mathrm{~d}^{-1}$ yields ${ }^{17} \Delta \sim 150$ per meg. Thus, as for NOP, the steady-state estimate of GOP is likely an underestimate of the true GOP in Phase 2.

\subsubsection{Sediment trap-based carbon fluxes}

During the cruise, total particulate organic carbon (POC) content (particles and swimmers) retained on filters gave a flux ranging from $31-56 \mathrm{mmol} \mathrm{C} \mathrm{m} \mathrm{m}^{-2} \mathrm{~d}^{-1}$. Estimates of the swimmer POC content ranged from 4-46 mmol C m${ }^{-2} \mathrm{~d}^{-1}$ (8-145\% of the total PC flux). The large spread in the swimmer corrections results in part because we only counted zooplankton in a small fraction of the water $(0.1 \mathrm{~L}$ out of $4.2 \mathrm{~L})$. Additionally, there is uncertainty in the conversion of the abundances of various zooplankton taxa to their carbon content (Nozais et al., 2005).

Considering that swimmer fluxes were not measured on one of the six deployments, there was a large range in swimmer PC estimates, and the calculated net PC flux on one day was negative (due to swimmer flux exceeding the net flux), we opted to apply the average swimmer correction of $21(18) \mathrm{mmol} \mathrm{C} \mathrm{m}^{-2} \mathrm{~d}^{-1}$ to each daily flux estimate. After this correction, the average PC export flux during the cruise was $21(19) \mathrm{mmol} \mathrm{C} \mathrm{m}^{-2} \mathrm{~d}^{-1}$ (range 10-36 mmol C m ${ }^{-2} \mathrm{~d}^{-1}$ ) and the PC flux was very similar in Phase 1 and Phase 2. In Figure 4-5, error estimates for each sediment trap are calculated by propagating error from the PC flux measured in the three collection tubes, and the uncertainty in the swimmer estimates. For the sediment traps, we consider the incubation beginning on 29 September to be more representative of Phase 2 because it began just a few hours prior to the start of Phase 2. As expected, the PC fluxes from all six traps measured at $50 \mathrm{~m}$ depth are lower than mixed layer NCP calculated from $\mathrm{O}_{2} /$ Ar and from ${ }^{15} \mathrm{NO}_{3}^{-}$uptake (Table 4-1). 


\subsection{Synthesis and comparison with prior work}

By integrating the results of all the methods, we gain a much more complete understanding of the ecosystem metabolism and carbon cycle state than we would achieve using just one method. We can quantify GPP, NPP, and NCP directly. By difference, we can quantify $R_{A}$ and $R_{H}$, the consumption of organic carbon by autotrophs and heterotrophs, respectively. Furthermore, we can evaluate the agreement between different methods of estimating the same parameters (e.g., $\mathrm{NCP}$ from $\mathrm{O}_{2} / \mathrm{Ar}$ and $\mathrm{NCP}$ from $\mathrm{NO}_{3}^{-}$uptake). For comparison purposes, we convert all methods to $\mathrm{C}$ units (Table 4-1) and present the results in an energy flow diagram (Figure 4-7), which displays the fate of GPP.

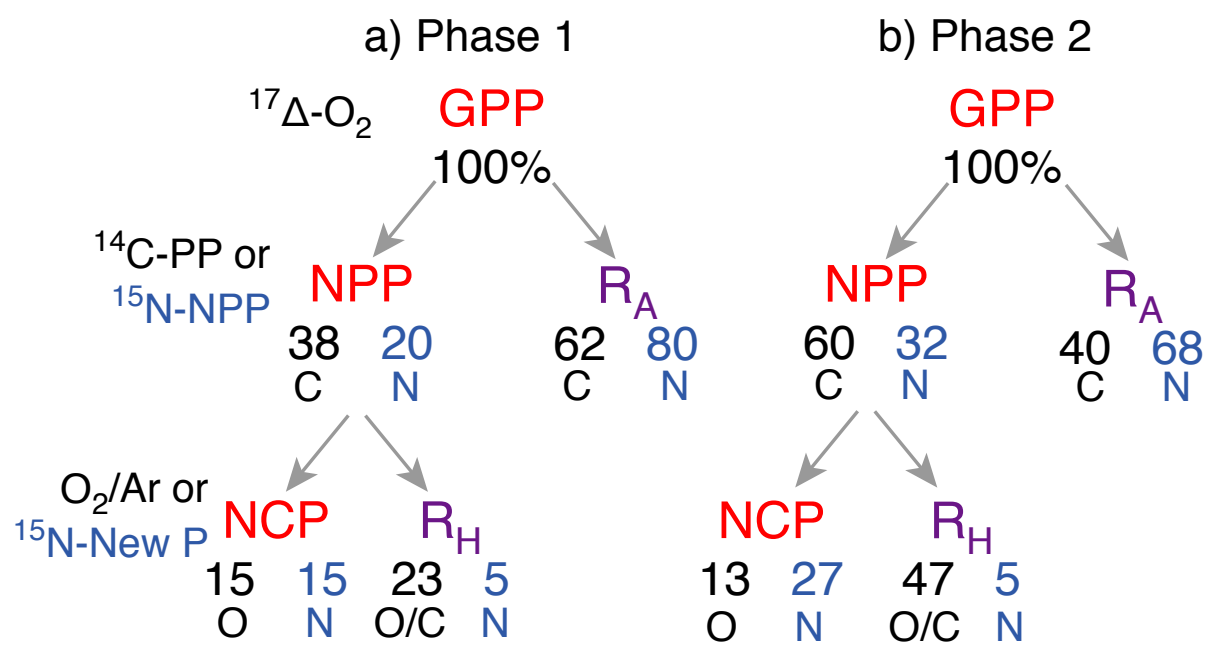

Figure 4-7: Energy flow diagram for (a) Phase 1 and (b) Phase 2, showing the percent of GPP that is respired by autotrophs $\left(R_{A}\right)$, respired by heterotrophs $\left(R_{H}\right)$, remains as NPP, and remains as NCP. ${ }^{15} \mathrm{~N}-\mathrm{NPP}$ is calculated from the sum of $\mathrm{NO}_{3}^{-}$and $\mathrm{NH}_{4}^{+}$uptake and ${ }^{15} \mathrm{~N}-\mathrm{New} \mathrm{P}$ is calculated from $\mathrm{NO}_{3}^{-}$uptake. All ratios are converted to $\mathrm{C}$ units using ratios as described in Table 4-1. In Phase 2, the values of NCP from $\mathrm{O}_{2}$ and GPP are likely an underestimate due to non-steady state conditions.

For example, if we assume that ${ }^{14} \mathrm{C}-\mathrm{PP}$ and the sum of ${ }^{15} \mathrm{NO}_{3}^{-}$and ${ }^{15} \mathrm{NH}_{4}^{+}$uptake both approximate NPP (Marra, 2002; Hendricks et al., 2005; Halsey et al., 2010), we can compare the results of these two methods. Mixed layer NPP from ${ }^{15} \mathrm{~N}$ was 47 and $46 \%$ lower than NPP from ${ }^{14} \mathrm{C}$ in Phase 1 and Phase 2 respectively. The discrepancy between the ${ }^{15} \mathrm{~N}$ and ${ }^{14} \mathrm{C}$ uptake rates suggests that other forms of $\mathrm{N}$ such as urea and other DON species were important N sources for phytoplankton (McCarthy, 1972; Bronk et al., 1994), and/or that a significant portion of the $\mathrm{N}$ taken up by phytoplankton was recycled during the $24 \mathrm{~h}$ incubations. Given that $\mathrm{N}$ is the limiting nutrient in Monterey Bay (Kudela and Dugdale, 2000), we expect dissolved organic matter and nutrient recycling to play a larger role in $\mathrm{N}$ uptake relative to $\mathrm{C}$. Temporal decoupling between $\mathrm{C}$ fixation and $\mathrm{N}$ uptake is another potential cause of discrepancies between simultaneous ${ }^{14} \mathrm{C}$ and ${ }^{15} \mathrm{~N}$ incubations. C fixation 
requires light but some phytoplankton, including diatoms which are a significant primary producer Monterey Bay, can uptake $\mathrm{N}$ in dark and light conditions (Hutchins and Bruland, 1995; Kudela and Dugdale, 2000; Clark et al., 2002). Although the absolute values of NPP calculated from ${ }^{15} \mathrm{~N}$ and ${ }^{14} \mathrm{C}$ are different due to the greater complexity of $\mathrm{N}$ cycling, the relative increase in productivity by both methods is nearly identical for Phase 2 relative to Phase 1.

We can also compare mixed layer $\mathrm{NCP}$ calculated from $\mathrm{O}_{2} / \mathrm{Ar}$ and the $\mathrm{NO}_{3}^{-}$uptake incubations. We assume that $\mathrm{NO}_{3}^{-}$uptake minus nitrification yields new production (Yool et al., 2007) and that new production is equivalent to NCP (Laws, 1991; Falkowski et al., 2003). In the mixed layer, nitrification decreased new production by $2 \%$. Nitrification rates were higher deeper in the water column and decreased euphotic zone nitrification (to $1 \%$ $\mathrm{I}_{o}$ ) by $3-45 \%$ (mean $17 \%$, data set DS3). In Phase 1, the methods agreed remarkably well, yielding $\mathrm{NCP}$ of $37(8)$ and $36(10) \mathrm{mmol} \mathrm{C} \mathrm{m} \mathrm{m}^{-2} \mathrm{~d}^{-1}$ from $\mathrm{NO}_{3}^{-}$uptake and $\mathrm{O}_{2} / \mathrm{Ar}$ respectively, despite the incubations measuring real-time uptake in bottles and the $\mathrm{O}_{2} / \mathrm{Ar}$ technique measuring productivity integrated over approximately one week (the residence time of $\mathrm{O}_{2}$ in the mixed layer). The agreement between the two methods supports our conclusion that $\mathrm{O}_{2}$ was at steady state in Phase 1. As expected, the particle flux to $50 \mathrm{~m}$ measured by the sediment traps $\left(21(19) \mathrm{mmol} \mathrm{C} \mathrm{m}^{-2} \mathrm{~d}^{-1}\right)$ was lower than the mixed layer NCP calculated by either method.

In Phase 2, new production increased to 65(10) $\mathrm{mmol} \mathrm{C} \mathrm{m}^{-2} \mathrm{~d}^{-1}$ whereas $\mathrm{O}_{2} /$ Ar-based NCP decreased to $30(12) \mathrm{mmol} \mathrm{C} \mathrm{m}^{-2} \mathrm{~d}^{-1}$, according to the steady state calculation. However, we believe that $\mathrm{O}_{2}$ was not in steady state during Phase 2 and therefore this calculation is likely an underestimate of the true NCP (section 4.6.4). We also emphasize that GPP in Phase 2 is likely an underestimate because the system was not at steady state. Therefore the magnitude of the apparent changes in the fraction of GPP going to NPP and NCP may be overestimated in Figure 4-7.

The frequent measurements of $\mathrm{O}_{2}$ in Phase 2 gave us insights into the biogeochemical dynamics which were not evident from the daily incubations. All of the 24-h incubations in Phase 2 were initiated at dawn with water from CTD profiles that had measurable nearsurface nitrate concentrations and low $\mathrm{O}_{2}$, characteristic of the recently-upwelled water. Evaluating $\mathrm{O}_{2}$ data from all of the CTD casts and underway measurements in Phase 2 showed a wider range of conditions, including both high nutrient, low $\mathrm{O}_{2}$ surface waters and low nutrient, high $\mathrm{O}_{2}$ surface waters. Thus the $\mathrm{O}_{2}$-based approaches, which were obtained regularly throughout the cruise, provide information on the submesoscale variability in productivity within our study site (Figures 4-4, C-1, and C-2). This high-frequency productivity data cannot be easily obtained via incubations because they are more labor intensive and because the time of day the incubation is initiated will affect the measured productivity (Pennington et al., 2015), confounding the detection of spatial variability. 
We can compare our $\mathrm{O}_{2} / \mathrm{Ar}$ and ${ }^{17} \Delta$-based estimates of $\mathrm{NCP}\left(36(10) \mathrm{mmol} \mathrm{C} \mathrm{m}^{-2} \mathrm{~d}^{-1}\right)$ and GPP (258(28) mmol C m$\left.{ }^{-2} \mathrm{~d}^{-1}\right)$ during Phase 1 with other recent studies using these methods in the California Current system. Munro et al. (2013) calculated NCP of 27(11) mmol $\mathrm{C} \mathrm{m}^{-2} \mathrm{~d}^{-1}$ and GPP of 168(61) mmol $\mathrm{C} \mathrm{m}^{-2} \mathrm{~d}$ during October 2006 in inshore California waters near Santa Barbara (termed the "north inshore" region in their study). Our Monterey Bay NCP is equivalent within uncertainty, but our GPP is higher. Our GPP results are very similar to their annually-averaged estimate of $232(86) \mathrm{mmol} \mathrm{C} \mathrm{m}^{-2} \mathrm{~d}^{-1}$. Haskell et al. (2016b) found mixed layer NCP to be $10(15) \mathrm{mmol} \mathrm{C} \mathrm{m}^{-2} \mathrm{~d}^{-1}$ in inshore California waters near Los Angeles during October 2013, lower than NCP during our study. GPP was 230(37) mmol C m${ }^{-2} \mathrm{~d}^{-1}$ during the same time period (W.Z. Haskell II, personal communication), which is similar to GPP during our study.

Additionally, Johnson (2010) calculated daily NCP using moored near-surface $\mathrm{O}_{2}$ and $\mathrm{NO}_{3}^{-}$sensors at $\mathrm{M} 1$ between April-August 2006. He found NCP ranged from approximately -24-48 and $-14-53 \mathrm{mmol} \mathrm{C} \mathrm{m}{ }^{-2} \mathrm{~d}^{-1}$ when calculated from $\mathrm{NO}_{3}^{-}$and $\mathrm{O}_{2}$ respectively. These results overlap with our Phase 1 estimates of NCP, but our Phase 2 estimate of NCP from $\mathrm{NO}_{3}^{-}$uptake incubations is somewhat higher than the corresponding range of results from $\left[\mathrm{NO}_{3}^{-}\right]$by Johnson (2010).

We can also look at ratios of different productivity measures to quantify the extent of recycling. If we assume that the $24 \mathrm{~h}$ incubations with ${ }^{14} \mathrm{C}$ approximate NPP (Marra, 2002), the ratio of GOP/NPP was 3.8(1.0) in Phase 1 and 2.4(0.5) in Phase 2. However, our ratio in Phase 2 is likely biased low due to the steady state calculation underestimating GOP. The GOP/NPP ratio in Phase 1 is similar to the GOP $/{ }^{14} \mathrm{C}-\mathrm{PP}$ ratio of 3.3 for phytoplankton growing on nitrate measured by Halsey et al. (2010), but somewhat higher than the ratio of 2.7 for gross $\mathrm{O}_{2}$ production to net $\mathrm{C}$ uptake observed in incubations from a global compilation of all JGOFS sites (Juranek and Quay, 2013). Munro et al. (2013) calculated a GOP/NPP ratio of $\sim 4.8-8.1$ in the north inshore region, using 6 h daytime ${ }^{14} \mathrm{C}$ incubations scaled to 24 $\mathrm{h}$ by multiplying by 1.81 (Eppley, 1992). Some of the discrepancy between the GOP/NPP ratio determined in our study and Munro et al. (2013) may be due to uncertainty in scaling the incubations from $6 \mathrm{~h}$ to $24 \mathrm{~h}$.

We also use the ef-ratio $=($ new production $) / \mathrm{NPP}=\mathrm{NCP} / \mathrm{NPP}$ (Laws et al., 2000) to quantify the fraction of net autotrophic production available for export, and the ratio of $\mathrm{NCP} / \mathrm{GPP}$ to quantify the fraction of gross autotrophic production available for export. In Phase 1 , the ratio of $\mathrm{NCP} / \mathrm{GPP}$ was 0.14 when calculated using ${ }^{17} \Delta$-GOP and NCP from either $\mathrm{NO}_{3}^{-}$uptake or $\mathrm{O}_{2} / \mathrm{Ar}$ (Figure 4-7). The ef-ratio was 0.77 and 0.84 in Phase 1 and Phase 2 respectively, when calculated from $\mathrm{NO}_{3}^{-}$and $\mathrm{NH}_{4}^{+}$uptake. It was 0.40 and 0.45 in Phase 1 and 2 respectively, when calculated from $\mathrm{NO}_{3}^{-}$uptake and ${ }^{14} \mathrm{C}-\mathrm{PP}$. In Phase 1 , the ef-ratio from $\mathrm{O}_{2} / \mathrm{Ar}$ and ${ }^{14} \mathrm{C}-\mathrm{PP}$ was 0.40 , identical to the result from $\mathrm{NO}_{3}^{-}$ uptake. In Phase 2, we have less confidence in the $\mathrm{O}_{2}$-based ef-ratio calculations because 
the system was not at steady state. The ef-ratio calculated from $\mathrm{N}$ uptake only is likely an overestimate throughout the cruise as it does not include additional sources of $\mathrm{N}$ such as urea (McCarthy, 1972; Eppley and Peterson, 1979). However, our ef-ratio values are similar to other measurements in Monterey Bay based on $\mathrm{NO}_{3}^{-}$and $\mathrm{NH}_{4}^{+}$uptake where ef-ratios > 0.5 are commonly observed (Kudela, 1995; Olivieri, 1996; Kudela and Dugdale, 2000); for example in a modeling study Olivieri and Chavez (2000) calculated an annually-averaged ef-ratio of 0.84 by this method.

Finally, the POC flux at $50 \mathrm{~m}$ was $\sim 58 \%$ of the mixed layer NCP in Phase 1 (from $\mathrm{O}_{2} / \mathrm{Ar}$ and from $\mathrm{NO}_{3}^{-}$uptake), and $32 \%$ of the mixed layer NCP in Phase 2 (from $\mathrm{NO}_{3}^{-}$ uptake), indicating that roughly half of the mixed layer NCP was respired between $\sim 14-50$ m. These results may suggest that the increase in mixed layer NCP during Phase 2 did not immediately generate an increase in export below the mixed layer. However, because we applied the same swimmer correction to all trap measurements, the apparent absence of a change in POC export during our time series must be interpreted with caution.

\subsection{Implications and future directions}

Coastal regions impacted by upwelling are a challenging environment in which to quantify oceanic productivity for a variety of reasons. By comparing the results of multiple methods we demonstrate the conditions under which productivity can be reliably determined by each method. To our knowledge, this is the first published study that has attempted to compare all of these methods from concurrent measurements in a coastal region.

Monterey Bay, like most of the ocean, is a N-limited system (Kudela and Dugdale, 2000; Moore et al., 2013). Some investigators have provided evidence that ${ }^{15} \mathrm{~N}$ additions can perturb $\mathrm{N}$ cycling and stimulate $\mathrm{N}$ uptake in incubations with low-nutrient water, especially for ammonium uptake experiments (Glibert and Goldman, 1981; Allen et al., 1996; Ward, 2005). Our results demonstrate that NCP calculated from ${ }^{15} \mathrm{NO}_{3}^{-}$uptake and from $\mathrm{O}_{2} / \mathrm{Ar}$ can give comparable results even in N-limited conditions, when the system is at steady state. The ${ }^{15} \mathrm{NO}_{3}^{-}$addition increased total $\mathrm{NO}_{3}^{-}$concentration by $\sim 51 \%$, which is significantly higher than typical recommendations to limit tracer addition to $\leq 10 \%$ of ambient concentrations (Dugdale and Goering, 1967). Despite the higher nutrient loadings, the two methods gave nearly identical results in Phase 1, when $\mathrm{NO}_{3}^{-}$concentrations were lower than in Phase 2, but the system was at steady state with respect to $\mathrm{O}_{2}$.

In Phase 2, our results demonstrate the advantages and disadvantages of different approaches. The incubation-based methods are better at recording short-term changes in productivity, while the high-frequency $\mathrm{O}_{2} / \mathrm{Ar}$ method integrates over longer timescales and enables the detection of submesoscale variability. We showed the importance of establishing whether a system is at steady state when using gas tracer-based approaches (Hamme et al., 
2012; Tortell et al., 2014; Wilson et al., 2015). If we only had a single time point measurement of $\mathrm{O}_{2} / \mathrm{Ar}$ and ${ }^{17} \Delta$, and did not have productivity estimates from other methods, we would have to assume that the system was at steady state despite having no evidence to support or refute this assumption. Instead, our high-resolution time-series measurements of $\mathrm{O}_{2} / \mathrm{Ar}$ revealed spatial and temporal variability over the scale of a few $\mathrm{km}$ or a few hours during Phase 2 (Figures 4-4, C-1, and C-2). These results provided evidence that the system was not at steady state and that multiple, biogeochemically unique water masses were present within our study area. Additionally, the incubations yield instantaneous, small-scale measurements of productivity and showed that productivity in the recently upwelled waters in Phase 2 was higher than productivity in Phase 1, but incubations were not performed with any water from Phase 2 that had higher $\mathrm{O}_{2}$ and lower nutrients.

At open ocean time-series sites, incubations conducted infrequently (e.g., monthly) can miss sporadic, short-term blooms and periods of high export, whereas investigators have argued that these blooms will be recorded more often by measurements of $\mathrm{O}_{2}$, which integrates over weeks in the open ocean (Karl et al., 2003; Juranek and Quay, 2005). However, in this field study, the phytoplankton bloom during Phase 2 was evident from the incubations but not the $\mathrm{O}_{2}$-based methods. During Phase 2, we sampled both inside and outside a filament of recently upwelled water where $\mathrm{O}_{2}$ was out of steady state. The higher nutrient concentrations in this filament initiated a phytoplankton bloom which pushed $\mathrm{O}_{2}$ further from steady state. The longer integration time of the $\mathrm{O}_{2}$-based method relative to the length of the timeseries and the submesoscale variability in surface water properties made it challenging to accurately quantify the increase in NCP and GPP in Phase 2 from these methods. A longer time-series would help to resolve these uncertainties. Also, if the different water masses had unique physical and/or biogeochemical properties that were continuously sampled in the un-

derway record (e.g., if the recently-upwelled water was significantly higher in salinity and/or if we had continuous underway measurements of $\left[\mathrm{NO}_{3}^{-}\right]$) we could potentially separate the time-series into different water parcels and separately quantify productivity in each parcel using our $\mathrm{O}_{2}$-based methods. In summary, we have demonstrated that ${ }^{15} \mathrm{NO}_{3}^{-}$incubations and gas tracer-based productivity estimates can give comparable results in an N-limited system and that by conducting time-series measurements of productivity by multiple methods, we can evaluate whether the system is at steady state.

\subsection{Acknowledgments}

Data critical to the conclusions of this study, including incubation results, gas measurements, nutrient measurements, and MATLAB code for calculating GOP is included in the Supplementary Information for this paper and on GitHub (http://github.com/caramanning/ calcGOP). MATLAB code for gas diffusivity, solubility, and air-sea fluxes is provided in 
Manning and Nicholson (2016). Additional data such as CTD profiles, microstructure profiles, and mooring data is available by contacting the lead author (cmanning@whoi.edu). The satellite SST data are available through the NASA Physical Oceanography Distributed Active Archive Center (http://podaac-www.jpl.nasa.gov). We thank Captain Andrew McKee of the $\mathrm{R} / \mathrm{V}$ Western Flyer and all cruise participants (crew and science team) for a successful cruise. Marguerite Blum (MBARI) analyzed the Winkler $\mathrm{O}_{2}$ and nutrient samples. Lisa Ziccarelli (MBARI) counted zooplankton swimmers in the sediment traps. Nick Welschmeyer (Moss Landing Marine Labs) provided the sediment traps. Kevin Cahill, Josh Curtice, Bill Jenkins, and Dempsey Lott (WHOI) performed noble gas sample extraction, analysis, and data reduction. Joanne Goudreau (WHOI) assisted with preparation of sample flasks. Zoe Sandwith (WHOI) trained CC Manning on $\mathrm{O}_{2}$ isotope sample processing and analysis. Chelle Gentemann (Earth and Space Research) provided helpful information on flagging of satellite SST data near fronts. Mooring data were collected and made available by the Biological Oceanography Group at MBARI. Funding for this work was provided by NSF awards OCE-1060840 to RHR Stanley, OCE-1129644 to DP Nicholson, OCE-1357042 to FP Chavez, NASA award NNX14AI06G to MR Fewings, the David and Lucile Packard Foundation through their generous annual donation to the Monterey Bay Aquarium Research Institute, an Ocean Ventures Fund award from the WHOI Academic Programs Office to CC Manning, and graduate scholarships from NSERC and CMOS to CC Manning. 


\section{Chapter 5}

\section{Changes in gross primary production, net community production, and air-water gas exchange during seasonal ice melt in the Bras d'Or Lake, a Canadian estuary}




\subsection{Abstract}

Sea ice is an important control on gas exchange and primary production in polar regions. We measured net oxygen production and gross oxygen production using near-continuous measurements of the $\mathrm{O}_{2} / \mathrm{Ar}$ gas ratio and discrete measurements of the triple isotopic composition of $\mathrm{O}_{2}$ in the Bras d'Or Lake, an estuary in Nova Scotia, Canada, as the bay transitioned from ice-covered to ice-free conditions. The volumetric gross oxygen production was $5.4\left({ }_{-1.6}^{+2.8}\right) \mathrm{mmol} \mathrm{O}_{2} \mathrm{~m}^{-3} \mathrm{~d}^{-1}$, similar at the beginning and end of the time-series, and likely peaked at the end of the ice melt period. Net oxygen production displayed more temporal variability and the system was on average net autotrophic during ice melt, and net heterotrophic following the ice melt. We performed the first field-based dual tracer release experiment in ice-covered water to quantify air-water gas exchange. The gas transfer velocity at $>90 \%$ ice cover was $6 \%$ of the rate for nearly ice-free conditions. Published studies have shown a wide range of results for gas transfer velocity in the presence of ice, and this study indicates that gas transfer through ice is much slower than the rate of gas transfer through open water. The results also indicate that both primary producers and heterotrophs are active in Whycocomagh Bay while it is covered in ice.

\subsection{Introduction}

The annual cycle of sea ice formation and melt regulates primary production and $\mathrm{CO}_{2}$ uptake and ventilation in polar regions. Ice alters the rate of air-water gas exchange, reduces the penetration of light into surface water, changes stratification and mixing processes, and harbors microbes and biogenic gases including $\mathrm{CO}_{2}$ (Cota, 1985; Loose et al., 2011b; Loose and Schlosser, 2011).

The issue of whether climate change will increase or decrease Arctic Ocean carbon uptake is a topic of considerable debate (Bates et al., 2006; Bates and Mathis, 2009; Cai, 2011). Global warming is causing dramatic reductions in sea ice cover and increases in freshwater inflow into the Arctic, which impacts Arctic ecosystems (ACIA, 2004; Vaughan et al., 2013). Because conducting field work in the Arctic is challenging, measurements of productivity and gas exchange are limited. Biogeochemical time-series observations resolving seasonal changes in productivity are particularly scarce in the Arctic (MacGilchrist et al., 2014; Stanley et al., 2015). Measurements at Palmer Station in Antarctica show a strong seasonality in biological productivity and carbon uptake associated with changes in light, physical mixing, and grazing and demonstrate the benefits of high-frequency sampling for quantifying $\mathrm{CO}_{2}$ uptake (Ducklow et al., 2013; Tortell et al., 2014; Goldman et al., 2015).

Parameterization of gas exchange in the presence of ice also remains highly uncertain. Many investigators have assumed that there is negligible gas transfer through ice and there- 
fore the gas transfer velocity can be linearly scaled as a function of the fraction of open water, multiplied by the open water gas transfer velocity (Takahashi et al., 2009; Legge et al., 2015; Evans et al., 2015; Stanley et al., 2015), however, this assumption is not well verified. A recent study by Butterworth and Miller (2016) concluded that gas transfer velocities in the Southern Ocean at 0-100 \% ice cover approximated the expected linear relationship with ice cover, but other studies report that gas exchange is reduced or enhanced in the presence of sea ice relative to a linear scaling based on the fraction of open water, including some studies measuring higher transfer velocities in ice-covered waters than in open water (Fanning and Torres, 1991; Else et al., 2011; Papakyriakou and Miller, 2011; Rutgers van der Loeff et al., 2014). Additional studies show that gas exchange in ice-covered waters cannot be predicted from wind speed alone, which may be a cause of the wide range of results (Loose et al., 2009a; Lovely et al., 2015).

In this study, we measured productivity and gas exchange over a 1-month period during and following ice melt in the Bras d'Or Lake, an estuary on Cape Breton Island in Nova Scotia, Canada. The Bras d'Or Lake consists of several interconnected channels and basins and has a surface area of $1070 \mathrm{~km}^{2}$ and an average depth of $\sim 30 \mathrm{~m}$ (maximum $280 \mathrm{~m}$ ) (Petrie and Raymond, 2002; Petrie and Bugden, 2002). The Bras d'Or Lake exchanges water with the Atlantic Ocean ocean primarily through the Great Bras d'Or Channel at the northeastern region of the estuary; this channel has a shallow (16 m deep) and narrow (0.3 $\mathrm{km}$ ) restriction at the mouth (Petrie and Raymond, 2002). We conducted the work for this study in Whycocomagh Bay, an enclosed embayment approximately $13 \mathrm{~km}$ long and $3 \mathrm{~km}$ wide, at the western end of the estuary, approximately $60 \mathrm{~km}$ from the open ocean (Figure $5-1)$. Whycocomagh Bay is separated from the rest of the Bras d'Or Lake by Little Narrows, a channel which is approximately $0.2 \mathrm{~km}$ wide and $0.5 \mathrm{~km}$ long. The bay is up to $40 \mathrm{~m}$ deep and Little Narrows channel is $\sim 15-20 \mathrm{~m}$ deep (Gurbutt and Petrie, 1995). Maximum ice cover is typically reached in early March and ice disappears most rapidly in April and has usually disappeared completely by the first week of May (Petrie and Bugden, 2002).

Nitrogen availability limits productivity in the Bras d'Or Lake for most of the year. N:P ratios are typically 6:1 or less, compared to the Redfield ratio of 16:1 (Redfield et al., 1963; Strain and Yeats, 2002). Inflowing water from Sidney Bight (Atlantic Ocean) is the most significant source of nutrients in all seasons (Strain and Yeats, 2002). Nutrient concentrations, especially nitrate, are thought to build up over winter due to mixing with deeper water and low phytoplankton growth rates, leading to a short phytoplankton bloom in spring as the water warms and ice melts, similar to the spring bloom in the nearby oceanic waters (Petrie and Raymond, 2002; Strain and Yeats, 2002; Greenan et al., 2004; Parker et al., 2007). However, a spring phytoplankton bloom in the Bras d'Or Lake has never been observed directly and nutrient concentrations have not been measured during winter (Parker et al., 2007). Concentrations of nitrate+nitrite in late spring 1996 were below $0.14 \mu \mathrm{M}$ 

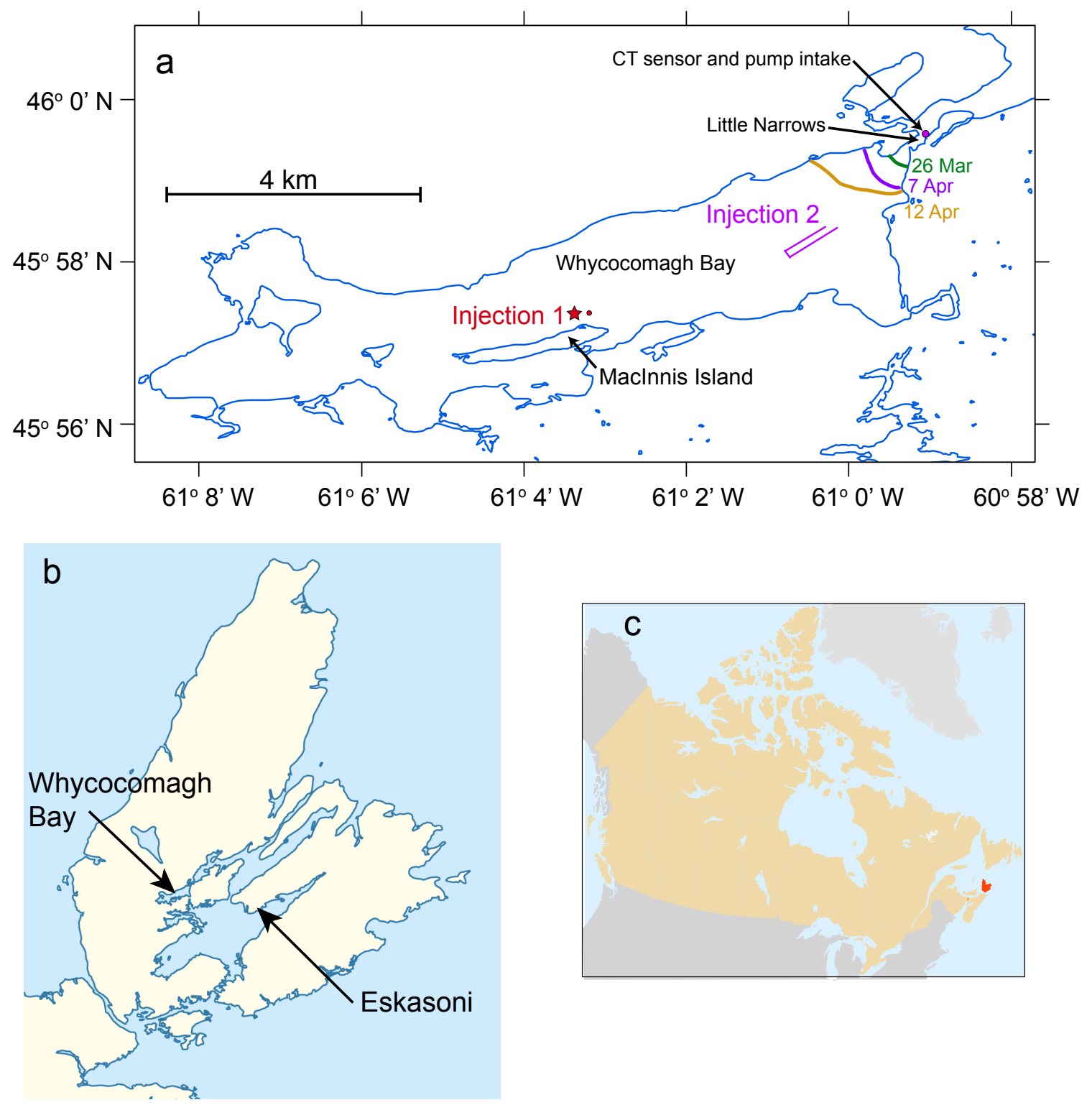

Figure 5-1: (a) Map of Whycocomagh Bay showing the locations of Injections 1 and 2 and the sampling equipment at Little Narrows. For Injection 1, the injection location is shown with a red star and the location where initial samples were collected is shown with a red circle. The colored lines labeled $26 \mathrm{Mar}, 7 \mathrm{Apr}$, and $12 \mathrm{Apr}$ show the location of the ice edge on these days. (b) Map of Cape Breton Island, showing the location of Whycocomagh Bay and Eskasoni (where wind speeds were obtained). (c) Map of Canada showing location of Cape Breton Island. Panels $(\mathrm{b}-\mathrm{c})$ were modified from an image created by Hanhil (https://commons.wikimedia.org/wiki/File:Canada_Nova_ Scotia_location_map_2.svg), "Canada Nova Scotia location map 2" as permitted under the CC-BY-SA 3.0 license, https://creativecommons.org/licenses/by-sa/3.0/legalcode. 
(the analytical detection limit) for 98 out of 114 samples and above $2 \mu \mathrm{M}$ in only 2 of 114 samples collected throughout the estuary (Strain, 2001). Ammonia concentrations had a median of $1.3 \mu \mathrm{M}$ during the same period (Strain, 2001; Strain and Yeats, 2002). The low chlorophyll and nitrate concentrations in these samples led Strain and Yeats (2002) to conclude that the spring bloom had already occurred. Nutrient concentrations remain low in summer and then increase due to mixing in fall. Given the low nutrient concentrations in spring and summer, regenerated nutrient sources drive the primary production in these seasons. For example, Geen and Hargrave (1966) demonstrated with incubation experiments that excretion of nutrients by zooplankton is needed to maintain the autotrophic biomass in summer.

We performed two dual tracer release experiments to quantify air-water gas exchange

by adding ${ }^{3} \mathrm{He}$ and $\mathrm{SF}_{6}$ to the mixed layer. The first tracer release experiment (Injection 1) occurred under near-complete ice cover and the second experiment (Injection 2) occurred in nearly ice-free conditions. We measured net oxygen production (NOP) and gross oxygen production (GOP) at Little Narrows while Whycocomagh Bay transitioned from completely ice-covered to ice-free conditions. GOP is the total amount of $\mathrm{O}_{2}$ generated by autotrophic microbes as a result of photosynthesis. NOP is GOP minus respiratory consumption of $\mathrm{O}_{2}$ by autotrophs and heterotrophs. The ratio of these two terms provides an estimate of the export efficiency, i.e. the fraction of autrophic production available for export from the mixed layer. The time-series approach allowed us to quantify non-steady state $\mathrm{O}_{2}$ fluxes, which can be a significant portion of the total $\mathrm{O}_{2}$ flux in many regions (Hamme et al., 2012; Tortell et al., 2014; Manning et al., 2016b). We quantify GOP with discrete measurements of the triple oxygen isotopic composition of $\mathrm{O}_{2}$ (Juranek and Quay, 2013) and NOP with near-continuous measurements of the $\mathrm{O}_{2} / \mathrm{Ar}$ saturation anomaly (Cassar et al., 2009). The advantages of the oxygen mass balance technique are that it is an in situ technique that does not perturb the system and integrates over larger spatiotemporal scales than bottle incubations. The challenge of the method is that physical $\mathrm{O}_{2}$ fluxes, including air-water gas exchange, must be quantified in order to separately quantify the biological $\mathrm{O}_{2}$ fluxes.

To our knowledge, this study is the first field-based experiment where the dual tracer technique is conducted in the presence of ice, and it adds to a limited number of in situ measurements of NOP and GOP during ice melt (Goldman et al., 2015; Stanley et al., 2015; Eveleth et al., 2016).

\subsection{Field work and analytical methods}

\subsubsection{Setup at Little Narrows}

We continuously sampled water at Little Narrows channel (Figure 5-1) over a 1-month period (25 March-28 April 2013). We moored a Goulds SB Bruiser 5-18 GPM submersible pump 
with intake at $\sim 0.5 \mathrm{~m}$ depth, placed inside a mesh filter bag to prevent large particles from clogging the pump, and a conductivity and temperature (CT) sensor (Sea-Bird Electronics SBE37) at $\sim 0.5 \mathrm{~m}$ depth. From the submersible pump, water flowed through flexible highpressure PVC tubing submerged underwater to a 3-port pressure-relief valve (on shore) and was then pumped along shore $(\sim 50 \mathrm{~m})$ to our sampling apparatus. The water passed through three 10" canister filters $(100,20$, and $5 \mu \mathrm{m}$ nominal pore size) and then into a sampling manifold containing three valves for distributing water for measurement of $\mathrm{O}_{2} / \mathrm{Ar}$ (by mass spectrometry), $\mathrm{SF}_{6}$ (by gas chromatography) and for discrete sampling. As discussed below, we sampled discretely for $\mathrm{SF}_{6},{ }^{3} \mathrm{He}$, and $\mathrm{O}_{2} / \mathrm{Ar}$ and the triple oxygen isotopic composition of $\mathrm{O}_{2}$. Excess water flowed through tubing back into the bay. We covered the tubing on shore and the filter canisters in foam insulation to minimize temperature changes in the water.

Due to the limited cable lengths, the CT sensor was initially were placed closer to shore than the water pump, but after obtaining a longer cable, we were able to co-locate the CT sensor with the water pump (beginning 12 April). We deployed a Nortek acoustic doppler current profiler (ADCP) at $4 \mathrm{~m}$ depth in the middle of the channel beginning on 7 April through the end of the time-series. The ADCP measurements are not used to interpret the results below because our measurements did not display any correlation with tidal cycles. However, a figure of the ADCP data is provided in Appendix C.

Salinity and temperature are needed to calculate the equilibrium concentration of each gas. For the discrete samples, we used the CT sensor temperature and salinity measurements beginning on 12 April (when we moved the CT to the same location as the pump). Prior to 12 April, we collected measurements with a YSI temperature and salinity probe from the water on shore and used these measurements as the temperature and salinity for the discrete samples. We determined the average warming through the underway line to be $0.37(0.22)$ ${ }^{\circ} \mathrm{C}$ based on comparisons between the temperatures from the CTD (in situ) and the YSI (on shore) and applied this offset to all YSI temperature measurements. Additionally, for the continuous $\mathrm{O}_{2} / \mathrm{Ar}$ data we used a temperature record from a thermocouple in the bucket because it had fewer gaps in time. We calibrated the thermocouple using an Aanderaa 4330 optode sensor which contains a temperature sensor (accuracy $\pm 0.03{ }^{\circ} \mathrm{C}$ ), which decreased the thermocouple temperature by $0.92{ }^{\circ} \mathrm{C}$ and then adjusted it by $0.37{ }^{\circ} \mathrm{C}$ to correct for warming.

We installed the gas chromatograph and mass spectrometer inside a garage connected to the Little Narrows Ferry building. The majority of the wet equipment was set up outside the garage adjacent to a window on the garage, which was used for transferring equipment and power cables between the outdoors and indoors. We deployed all equipment in the water to the east (oceanward) of the Little Narrows cable ferry which periodically crosses the channel and operates 24 hours per day. There were no trends in the data consistently correlated to the position of the ferry within the channel. We collected conductivity, temperature and 
depth (CTD) profiles with a SBE 19Plus sensor at Little Narrows, usually by boat using a winch, but occasionally by lowering the CTD by hand on a rope from the Little Narrows cable ferry. The GPS-equipped boat enabled us to map out the location of the ice edge nearest to Little Narrows, to perform the second tracer injection, and to sample after the tracer injection.

\subsubsection{Tracer injections}

Our approach was to dissolve the tracer mixture $\left({ }^{3} \mathrm{He} / \mathrm{SF}_{6}\right)$ in Whycocomagh Bay, and sample continuously at Little Narrows, a constriction at the mouth of the bay. The net surface flow within Whycocomagh Bay, Little Narrows, and St. Patrick's Channel is toward the ocean due to the substantial freshwater inputs to the bay (Gurbutt and Petrie, 1995; Yang et al., 2007) and therefore tracer dissolved within the bay at the surface will eventually pass through Little Narrows (or be ventilated to the atmosphere). We conducted two tracer injections, resulting in an estimate of the gas transfer velocity for two extremes: nearcomplete ice cover, and essentially ice-free conditions.

The first injection occurred through a hole in the ice from 30-31 March, near MacInnis Island (Figures 5-1a and D-3). Approximately $0.11 \mathrm{~mol} \mathrm{SF}_{6}$ and $4.0 \times 10^{-4} \mathrm{~mol}{ }^{3} \mathrm{He}$ was diluted by a factor of 50 with $\mathrm{N}_{2}$ and then bubbled using a manifold, over a 21-hour period within the mixed layer. We sampled for initial ${ }^{3} \mathrm{He}$ and $\mathrm{SF}_{6}$ concentrations after terminating the tracer addition, by drilling a separate hole near the injection site. Subsequently, we sampled the tracer as it flowed through Little Narrows from 7-11 April. From 31 March-11 April, the bay was nearly completely full of ice, with a small opening near Little Narrows.

The second injection occurred by boat on the morning of 19 April. By this time, the bay was nearly ice-free and all tracer from the previous experiment had passed through and/or been ventilated to the atmosphere such that the tracer concentrations at Little Narrows were below detection. While the boat was moving, we used the same manifold as for Injection 1 to bubble approximately approximately $4.4 \mathrm{~mol} \mathrm{SF}_{6}$ and $0.021 \mathrm{~mol}{ }^{3} \mathrm{He}$, diluted by a factor of four with $\mathrm{N}_{2}$ into the mixed layer (Figure 5-1a). The injection lasted $40 \mathrm{~min}$. We detected the tracers at Little Narrows beginning on 20 April 23:30 and measured the change in the ratio between 20 April-23 April. We collected samples within the tracer patch by boat on 19 April in the afternoon, but all ${ }^{3} \mathrm{He}$ samples had air contamination or were lost during sample extraction.

\subsubsection{Measurement of $\mathrm{O}_{2} / \mathrm{Ar}$ and the triple oxygen isotopic composition of $\mathrm{O}_{2}$}

We set up an equilibrator inlet mass spectrometer (EIMS) for measurement of $\mathrm{O}_{2} / \mathrm{Ar}$ similarly to the system described in Cassar et al. (2009). However, we used a larger membrane 
contactor cartridge (Liqui-Cel MiniModule $1.7 \times 5.5$ ) because the design was more robust (less prone to flooding the headspace) than the Liqui-Cel MicroModule $0.75 \times 1$ used by Cassar et al. (2009). The water flow rate through the cartridge was $\sim 1.5 \mathrm{~L} \mathrm{~min}^{-1}$. We attached a custom fitting with a female Luer-Lok cap and a capillary adapter to the upper headspace sampling port and the lower sampling port was left capped (closed). From 15-28 April we also measured the concentration of $\mathrm{O}_{2}$ using an Aanderaa optode, model 4330. The optode was not calibrated because equipment for Winkler titrations was not available in the field and the optodes drift during storage/transport (Bittig and Körtzinger, 2015; Johnson et al., 2015). Although the profiling CTD had an $\mathrm{O}_{2}$ sensor, it was malfunctioning during the time-series and the data from it could not be used.

For $\mathrm{O}_{2} / \mathrm{Ar}$ and the triple oxygen isotopic composition of $\mathrm{O}_{2}$, we collected samples in pre-evacuated, pre-poisoned glass flasks from a spigot in the water pumped to shore, or for shipboard sampling, using a small submersible water pump. Analysis occurred within $\sim 6$ months of flask evacuation and 4 months of sample collection at the Woods Hole Oceanographic Institution with a Thermo Fisher Scientific MAT 253 isotope ratio mass spectrometer, following the method of Barkan and Luz (2003) with modifications as described in Stanley et al. (2010, 2015).

The precision of the discrete samples, calculated based on the standard deviation of equilibrated water samples run daily along with the environmental samples was 0.011 and $0.020 \%$ for $\delta^{17} \mathrm{O}$ and $\delta^{18} \mathrm{O}$ respectively, 5.6 per meg for ${ }^{17} \Delta$, and $0.07 \%$ for $\mathrm{O}_{2} /$ Ar. The mean difference between the EIMS and discrete samples was $0.05 \%$, and the mean magnitude of the difference was $0.35 \%$. Because the mean difference between the EIMS and discrete samples is smaller than the precision of the discrete samples, the EIMS measurements are not adjusted to match the $\mathrm{O}_{2} /$ Ar measurements.

\subsubsection{Measurement of $\mathrm{SF}_{6}$}

For $\mathrm{SF}_{6}$, we collected $\sim 20 \mathrm{~mL}$ water samples in $50 \mathrm{~mL}$ glass gas-tight syringes, then added $\sim 20 \mathrm{~mL}$ of nitrogen and allowed the samples to be shaken for $10 \mathrm{~min}$ to achieve equilibration between the headspace and water (Wanninkhof et al., 1987). After the water equilibrated to room temperature, we injected $1 \mathrm{~mL}$ of the headspace into an SRI-8610C gas chromatograph with an electron capture detector, heated to $300{ }^{\circ} \mathrm{C}$ (Lovely et al., 2015). We calibrated the detector response using a $150 \mathrm{ppt} \mathrm{SF}_{6}$ standard (balance $\mathrm{N}_{2}$ ). Unfortunately, a gas cylinder filled with a lower concentration of $\mathrm{SF}_{6}(10 \mathrm{ppt})$ arrived to the field site empty and could not be used as a second calibration standard.

We also operated an automated gas extraction system at Little Narrows which sampled nearly every hour. The system recirculated a $118 \mathrm{~mL}$ loop of water through a membrane contactor, and the headspace of the membrane contactor was under vacuum, causing the gas to be extracted from the water into the headspace. This system enabled determination of 
when the tracer was flowing through Little Narrows and helped inform the timing of discrete sample collection.

$\mathrm{SF}_{6}$ equilibrium solubility concentrations are calculated following Bullister et al. (2002) and diffusivity is from King and Saltzman (1995). Precision of the system, based on the standard deviation of measurements of the $150 \mathrm{ppt}$ standard, was $7 \%$. We assume a dry atmospheric mole fraction of 8 ppt for $\mathrm{SF}_{6}$ (Bullister, 2015).

\subsubsection{Measurement of ${ }^{3} \mathrm{He}$}

For ${ }^{3} \mathrm{He}$ analysis, we collected samples in copper tubes, mounted in aluminum channels and sealed the samples at each end using clamps. Sample analysis occurred at the Lamont Doherty Earth Observatory, using a VG5400 mass spectrometer for ${ }^{3} \mathrm{He}$ and ${ }^{4} \mathrm{He}$ concentration (Ludin et al., 1998). We used He solubility from Lott and Jenkins (personal communication, 2015) and diffusivity from Jähne et al. (1987a). The Lott and Jenkins is solubility 2 \% higher than published data from Weiss (1971). The He solubility is for bulk He and we calculate the ${ }^{3} \mathrm{He}$ solubility using an atmosperic mole ratio $M\left({ }^{3} \mathrm{He}\right) / M\left({ }^{4} \mathrm{He}\right)=1.399 \times 10^{-6}$ (Mamyrin et al., 1970; Porcelli et al., 2002), although some more recent results indicate the current ratio may be slightly lower, $\simeq 1.390 \times 10^{-6}$ (Brennwald et al., 2013b). We use the He equilibrium isotopic fractionation as described in Benson and Krause (1980b). Error for ${ }^{3} \mathrm{He}$ sample analysis (combined precision and accuracy) was $\leq 2 \%$ of the measured ${ }^{3} \mathrm{He}$ excess concentration above equilibrium.

\subsection{Calculations, results, and discussion}

The three goals of our experiment were to 1) quantify gas transfer velocity by dual tracer release, 2) quantify gross oxygen production from the triple isotopic composition of $\mathrm{O}_{2}$, and 3) quantify net oxygen production from $\mathrm{O}_{2} / \mathrm{Ar}$. We begin by discussing the hydrographic characteristics of the study area and then describe the calculations, results, and interpretation for each of the three goals, in sequence.

\subsubsection{Hydrography}

We began sampling $\mathrm{O}_{2}$ at Little Narrows on 25 March, when Whycocomagh Bay was nearly (>95\%) full of ice, and completed sampling on 28 April, when the bay was completely icefree (Figure 5-2). The surface ice cover retreated most rapidly between 16-18 April and was completely gone by 22 April or perhaps even earlier. Figure 5-2 shows 18 April and 23 April; MODIS images on 22 April were also ice-free (but more blurry, so are not shown in the figure) and we estimated ice cover to be $<10 \%$ in the bay during surveys by boat during daytime on 20 April. The ice was likely melting even at the beginning of the time-series since the surface water temperature was always above the freezing temperature of water. Changes in 
surface ice cover and total ice volume are both important factors during the study; changes in ice volume will affect stratification and convection in the mixed layer and the surface ice cover controls the rate of gas exchange (Smith and Morison, 1993; Butterworth and Miller, 2016)

CTD profiles at Little Narrows channel near the water pump intake showed substantial changes in stratification during the time-series (Figure 5-3). From 25 March through 8 April the water column was strongly stratified and we estimated the mixed layer depth to average $0.8(0.3) \mathrm{m}$. During this period, it was often difficult to determine the exact mixed layer depth because the mixed layer depth was similar to the length of the CTD and obtaining a stable CTD response so close to the surface was challenging. Following 8 April, the mixed layer deepened and warmed and its salinity increased, likely due to convection and heating following sea ice melt. For this period, we defined the mixed layer depth as the first depth where the density is $1 \mathrm{~kg} \mathrm{~m}^{-3}$ greater than the value at $1 \mathrm{~m}$. The mixed layer reached a maximum of $3.0 \mathrm{~m}$ on 23 April and then shoaled by the end of the time-series on 28 April. On most days, the density profile and mixed layer depth were driven by stratification in salinity, but for the final profile on 28 April the mixed layer depth was determined by a combination of temperature and salinity stratification due to heat uptake by the surface water following the ice melt. These changes in mixed layer depth must be considered in order to interpret the $\mathrm{O}_{2}$ measurements and to quantify the gas transfer velocity.

\subsubsection{Gas transfer velocity}

\section{Calculation}

We calculate the gas transfer velocity using the dual tracer approach (Watson et al., 1991b; Wanninkhof et al., 1993). For each experiment, we dissolved a mixture of ${ }^{3} \mathrm{He}$ and $\mathrm{SF}_{6}$ in the water, both of which are normally present at very low ambient concentrations, and then measured the change in the ratio of the two gases as a function of time. Measuring two tracers enables correction for dilution and mixing, which reduces the excess concentrations of both gases (relative to air-water equilibrium) but does not change their ratio. Over time, the concentrations of both gases decay toward air-water equilibrium as gas is ventilated to the atmosphere through gas exchange. Because the molecular diffusivity of ${ }^{3} \mathrm{He}$ is $8-9$ times higher than $\mathrm{SF}_{6},{ }^{3} \mathrm{He}$ is lost to the atmosphere more rapidly than $\mathrm{SF}_{6}$ and therefore the ${ }^{3} \mathrm{He}: \mathrm{SF}_{6}$ ratio decreases with time. The ratio of the gas transfer velocity for the two gases is expressed as

$$
\frac{k_{3} \mathrm{He}}{k_{\mathrm{SF}_{6}}}=\left(\frac{S c_{3} \mathrm{He}}{S c_{\mathrm{SF}_{6}}}\right)^{-n}
$$

where $k$ is the gas transfer velocity $\left(\mathrm{m} \mathrm{d}^{-1}\right)$ and $S c$ is the Schmidt number (unitless), defined as the kinematic viscosity of water divided by the molecular diffusivity of the gas in water, 

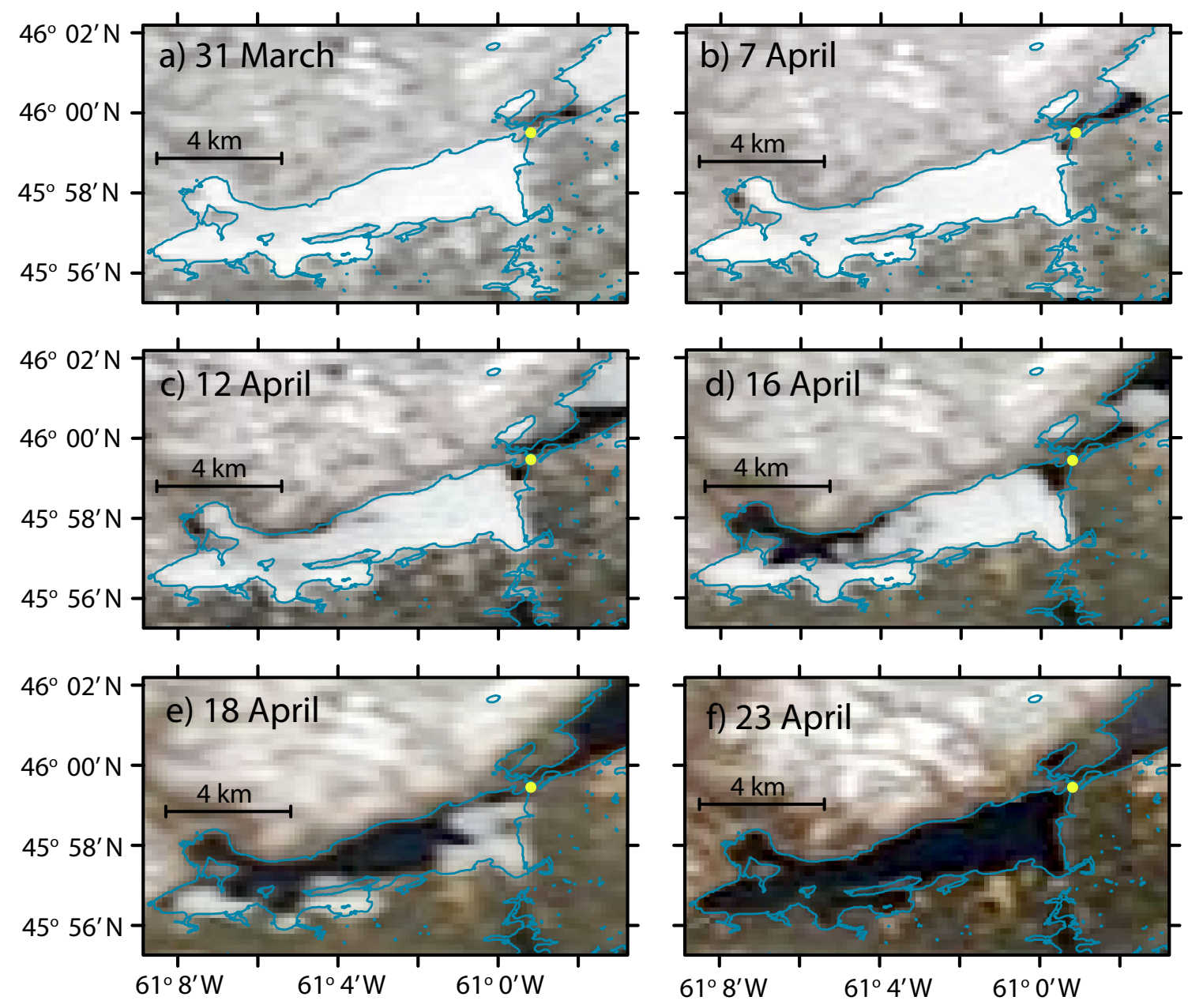

Figure 5-2: MODIS Terra true color images showing changes ice cover during the timeseries between 31 March to 23 April. Little Narrows is indicated with a yellow circle on all images. Ice cover retreated most rapidly between 12 April-20 April. Shoreline data (blue lines) is from GeoGratis/Natural Resources Canada (http://geogratis.gc.ca) and satellite data is from NASA Worldview (http://worldview.earthdata.nasa.gov).

and $n$ is an empirical exponent, typically between 0.5-0.67 (Jähne et al., 1984; Liss and Merlivat, 1986). Using a time-series of measurements of the two gases, the gas transfer velocity for ${ }^{3} \mathrm{He}$ is calculated as

$$
k_{3_{\mathrm{He}}}=h \frac{d}{d t}\left(\frac{\left.\ln \left(\left[{ }^{3} \mathrm{He}\right]_{e x c} /\left[\mathrm{SF}_{6}\right]_{\text {exc }}\right)\right]}{1-\left(S c_{\mathrm{SF}_{6}} / S c_{3} \mathrm{He}\right)^{-n}}\right) .
$$

Here $\left[{ }^{3} \mathrm{He}\right]_{e x c}=\left[{ }^{3} \mathrm{He}\right]_{\text {meas }}-\left[{ }^{3} \mathrm{He}\right]_{e q}$ where $\left[{ }^{3} \mathrm{He}\right]_{e x c}$ is the ${ }^{3} \mathrm{He}$ excess concentration, $\left[{ }^{3} \mathrm{He}\right]_{\text {meas }}$ is the measured concentration and $\left[{ }^{3} \mathrm{He}\right]_{e q}$ is the equilibrium concentration (calculated as a function of temperature and salinity). $\left[\mathrm{SF}_{6}\right]_{\text {exc }}$ is defined analogously. 
a) Salinity [PSS]

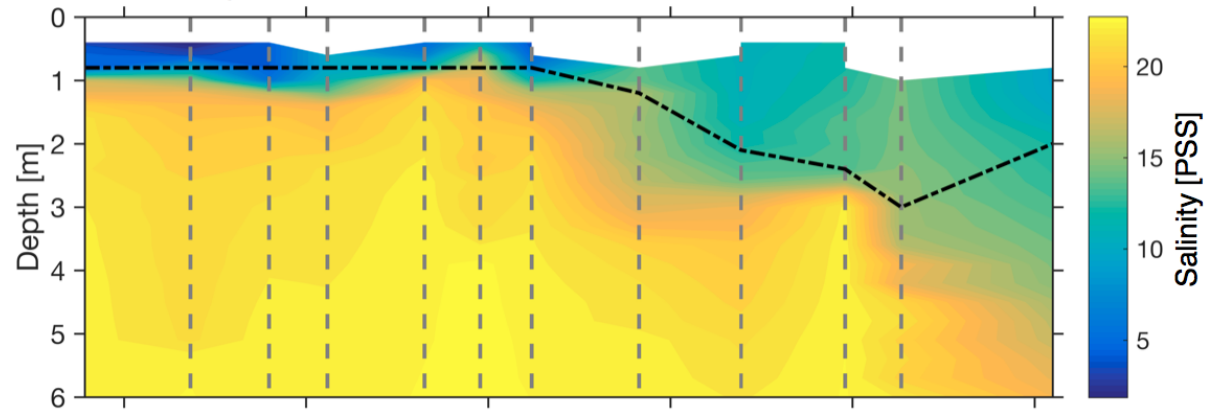

b) Temperature $\left[{ }^{\circ} \mathrm{C}\right]$

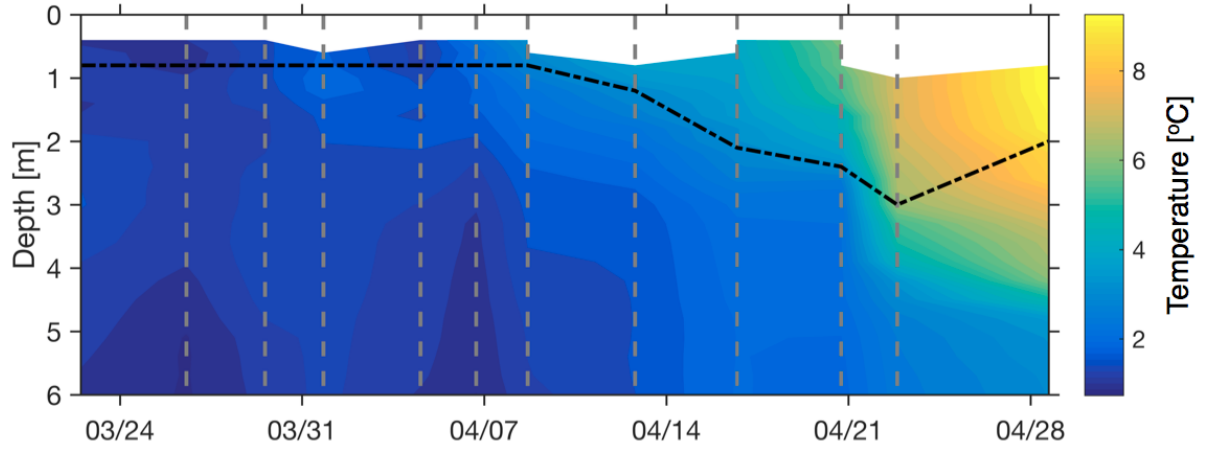

Figure 5-3: (a) Salinity and (b) temperature profiles measured at Little Narrows. The vertical grey dashed lines indicate the timing of CTD casts and the black dot-dashed line shows the mixed layer depth.

We can write the analytical solution to equation 5.2 as (Ho et al., 2011b)

$$
\left(\frac{\left[{ }^{3} \mathrm{He}\right]_{e x c}}{\left[\mathrm{SF}_{6}\right]_{e x c}}\right)_{t}=\left(\frac{\left[{ }^{3} \mathrm{He}\right]_{e x c}}{\left[\mathrm{SF}_{6}\right]_{e x c}}\right)_{t-1} \exp \left(-\frac{k_{3 H e} \Delta t}{h}\left[1-\left(\frac{S c_{\mathrm{SF}_{6}}}{S c_{3}{ }_{\mathrm{He}}}\right)^{-n}\right]\right) .
$$

Using this equation and a cost function, we can find the value of $k_{3} \mathrm{He}$ that minimizes the error between the measured and modeled $\left[{ }^{3} \mathrm{He}\right]_{e x c} /\left[\mathrm{SF}_{6}\right]_{e x c}$. Once $k_{3} \mathrm{He}$ is known, we can calculate $k$ for any other gas using equation 5.1 by substituting $S c_{\mathrm{SF}_{6}}$ for the Schmidt number of the gas of interest. For example, for $S c=600$ (the Schmidt number for $\mathrm{CO}_{2}$ at $20{ }^{\circ} \mathrm{C}$ in freshwater)

$$
k_{600}=k_{3 \mathrm{He}}\left(\frac{600}{S c_{3} \mathrm{He}}\right)^{-n} .
$$

For this study, we use a Schmidt number dependence of $n=0.5$ which is appropriate for wavy, unbroken water surfaces (no bubble entrainment) (Jähne et al., 1984; Liss and Merlivat, 1986; Jähne et al., 1987a; Ho et al., 2011b). At Little Narrows, we observed that tidal currents generated surface waves, even at low wind speeds. These waves produce nearsurface water turbulence which is the ultimate driver of air-water gas exchange (Jähne et al., 1987b; Wanninkhof et al., 2009). 


\section{Results}

We obtained significantly different gas transfer velocities for Injection 1, which was sampled while the basin was essentially full of ice (31 March-10 April) and Injection 2, which was sampled when the basin was nearly ice-free (20-23 April).

We used equation 5.3 to model the measurements. We started the model at the time of the first measurement, initialized it with an initial excess concentration ratio and ran it through time for the duration of the injection. We selected the value of $k_{3} \mathrm{He}$ yielding the smallest root mean square deviation (RMSD) between the measured ratio and modeled ratio for each injection. We ran the model 1000 times using a Monte Carlo simulation where the measured excess concentration ratios, including the initial condition, are varied with a Gaussian distribution, with the standard deviation being the estimated measurement error in the ratio $(7.3 \%)$.

We assume a constant $S c_{3}{ }_{\mathrm{He}} / S c_{\mathrm{SF}_{6}}$ and mixed layer depth $(h)$ for each injection. In actuality, during each injection $S c_{3} \mathrm{He}$ varies by $\sim 1 \%$ and the ratio of the Schmidt numbers varies by less than $0.2 \%$, so this is a small source of error.

We assume a mixed layer depth of $0.8(0.3) \mathrm{m}$ for Injection 1. This depth is consistent with the salinity profiles at Little Narrows (Figure 5-3a) between 31 March and 8 April (between 0.6-1.0 $\mathrm{m}$ depth), as well as measurements with a hand-held temperature probe at the site of Injection 1 which indicated that the mixed layer depth was between $0.75-1 \mathrm{~m}$.

For Injection 1, the excess $\mathrm{SF}_{6}$ and ${ }^{3} \mathrm{He}$ concentrations were reduced by two orders of magnitude by the time the tracer reached Little Narrows (7-11 days after injection). The tracer ratio did not display a consistent decrease over the three days we sampled it at Little Narrows, likely in part due to the substantially lower gas transfer velocity. The best fit to all the measurements was $k_{600}=0.0457(0.0051) \mathrm{m} \mathrm{d}^{-1}$, with the uncertainty the standard deviation of the distribution of $k_{600}$ from the Monte Carlo simulation (Figure 5-4). We avoided collecting discrete tracer samples when the tides were flowing into Whycocomagh Bay, based on visual observation of surface currents, to ensure that the gas transfer velocity reflected the ice coverage within the bay and was not skewed by the open water east of Little Narrows.

The mixed layer appeared to deepen between the CTD profiles on 8 April 16:08 and 12 April 19:13, and it is possible the mixed layer depth on 9-10 April may have been deeper than the estimate of $0.8(0.3) \mathrm{m}$. However, if this were the case we would expect $k_{600}$ (calculated assuming a constant mixed layer depth) to be lowest when calculated over the longest time period, using the sample collected on 10 April. Instead, the gas transfer velocity was actually the lowest when integrated to 9 April (the excess tracer ratio appears above the best-fit line) and second lowest on 8 April. Since the gas transfer velocities for Injection 1 integrate over 7-10 days, any change in mixed layer depth during the last 1-2 days will have a small effect on the calculated $k$. 


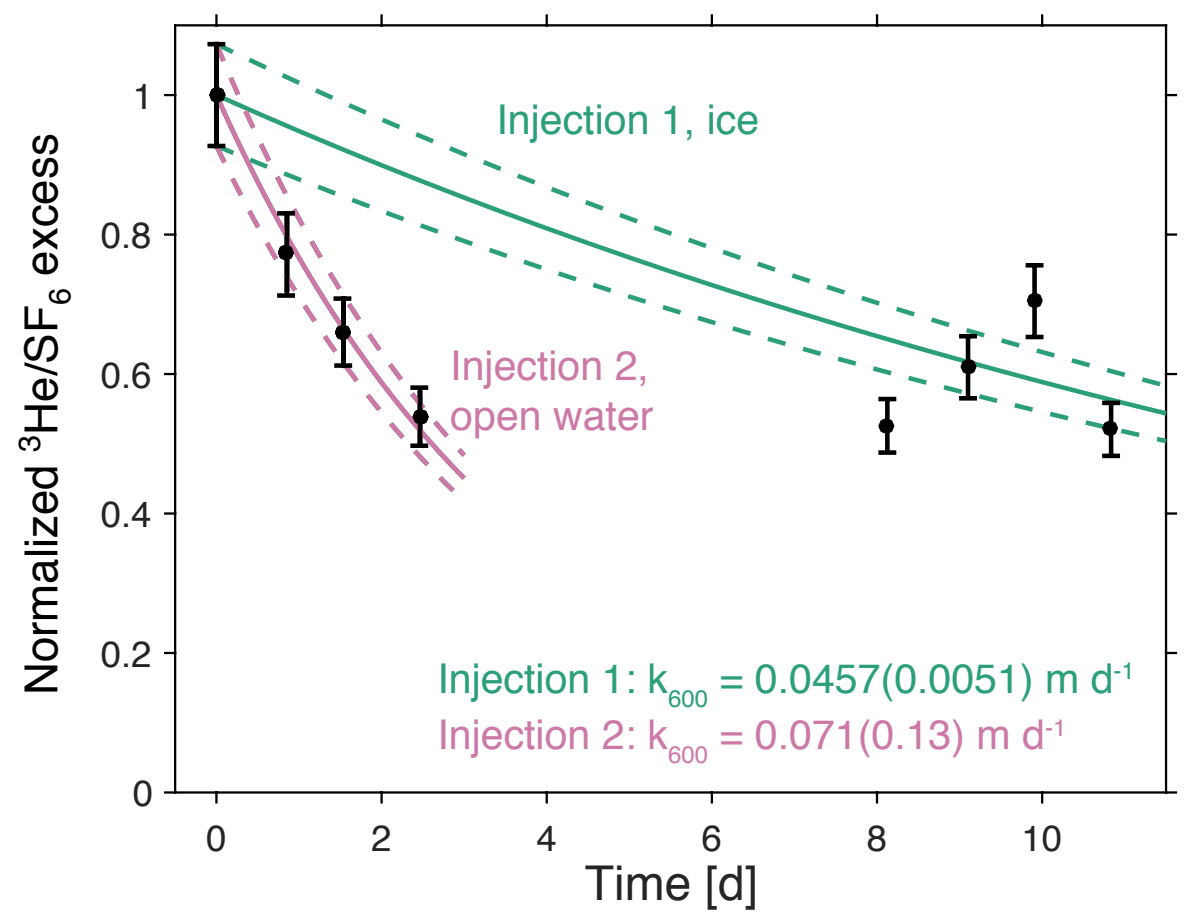

Figure 5-4: Measured and modeled ratio of excess ${ }^{3} \mathrm{He} / \mathrm{SF}_{6}$, normalized to the initial measured ratio for each injection. The modeled excess ratio is calculated using the $k_{3} \mathrm{He}$ that minimizes error between the model and measurements. Model results are shown for the model initialized with the initial measured concentration (solid lines), and starting one standard deviation above or below the measured initial concentration (based on an error of $7.3 \%$ in the tracer ratio, dashed lines). 
Table 5-1: Data for determination of the gas transfer velocity

\begin{tabular}{|c|c|c|c|c|c|c|}
\hline \multicolumn{7}{|c|}{ Injection 1} \\
\hline Date & Time & $\begin{array}{l}\text { Salinity }^{a} \\
\text { (PSS) }\end{array}$ & $\begin{array}{l}\text { Temperature }{ }^{a} \\
\left({ }^{\circ} \mathrm{C}\right)\end{array}$ & $\begin{array}{l}\delta^{3} \mathrm{He} \\
(\%)\end{array}$ & $\begin{array}{l}{\left[\mathrm{SF}_{6}\right]} \\
\left(10^{-12} \mathrm{~mol} \mathrm{~L}^{-1}\right)\end{array}$ & $\begin{array}{l}{\left[{ }^{3} \mathrm{He}\right]_{e x c} /\left[\mathrm{SF}_{6}\right]_{e x c}} \\
\left(\mathrm{~mol} \mathrm{~mol}{ }^{-1}\right)\end{array}$ \\
\hline 31 March & $15: 00$ & 0.9 & 2.1 & 17176 & 66.6 & 0.00788 \\
\hline 7 April & 18:00 & 13.2 & 2.6 & 13.8 & 0.152 & 0.00411 \\
\hline 8 April & $17: 27$ & 8.4 & 2.0 & 56.9 & 0.423 & 0.00480 \\
\hline 9 April & $12: 47$ & 9.0 & 2.1 & 39.0 & 0.289 & 0.00553 \\
\hline 10 April & 11:02 & 9.3 & 1.8 & 12.6 & 0.115 & 0.00405 \\
\hline \multicolumn{7}{|c|}{ Injection 2} \\
\hline Date & Time & $\begin{array}{l}\text { Salinity }^{a} \\
\text { (PSS) }\end{array}$ & $\begin{array}{l}\text { Temperature } \\
\left({ }^{\circ} \mathrm{C}\right)\end{array}$ & $\begin{array}{l}\delta^{3} \mathrm{He} \\
(\%)\end{array}$ & $\begin{array}{l}{\left[\mathrm{SF}_{6}\right]} \\
\left(\mathrm{mol} \mathrm{L}^{-1}\right)\end{array}$ & $\begin{array}{l}{\left[{ }^{3} \mathrm{He}\right]_{e x c} /\left[\mathrm{SF}_{6}\right]_{e x c}} \\
\left(\mathrm{~mol} \mathrm{~mol}{ }^{-1}\right)\end{array}$ \\
\hline 20 April & $23: 30$ & 11.18 & 5.64 & 310.3 & 3.96 & 0.00227 \\
\hline 21 April & $20: 00$ & 14.91 & 6.49 & 91.8 & 1.60 & 0.00176 \\
\hline 22 April & $12: 27$ & 14.64 & 7.16 & 43.6 & 0.938 & 0.00150 \\
\hline 23 April & $10: 40$ & 11.01 & 5.73 & 6.5 & 0.299 & 0.000799 \\
\hline
\end{tabular}

${ }^{a}$ For Injection 1, we measured temperature and salinity with a YSI probe to a precision of one decimal place (because the CTD was not yet co-located with the water pump and could not be used to sample under ice). For Injection 2, we measured temperature with a calibrated thermocouple and salinity with the in situ CTD, to a precision of two decimal places.

For Injection 2, we use a mixed layer depth of 2.7(0.3) $\mathrm{m}$ based on CTD profiles at Little Narrows on 20 April and 23 April, which had mixed layer depths of 2.4 and 3.0 $\mathrm{m}$, respectively (Figure 5-3a). Calculation of the gas transfer velocity for Injection 2 was relatively straightforward as the ratio of excess ${ }^{3} \mathrm{He} / \mathrm{SF}_{6}$ steadily decreased over the five measurements (Table 5-1). The best fit to all four measurements was $k_{600}=0.71(0.13) \mathrm{m}$ $\mathrm{d}^{-1}$ (Figure 5-4).

If we use published helium solubility from Weiss (1971) instead of the unpublished data of Lott and Jenkins (2015, personal communication) we calculate a transfer velocity that is $8 \%$ lower for Injection 1 and $0.4 \%$ lower for Injection 2 .

\section{Discussion}

The gas transfer velocity calculated for Injection 1 is the effective gas transfer velocity $\left(k_{e f f}\right)$; it averages the gas transfer velocity through ice $\left(k_{i c e}\right)$, weighted by the time the tracer spent under ice, and the gas transfer velocity for open water $(k)$, weighted by the time the tracer spent in open water (Loose et al., 2014). In partially-ice covered waters, the effective gas transfer velocity is sometimes calculated as

$$
k_{e f f}=(f) k+(1-f) k_{i c e}
$$


where $f$ is the fraction of open water (Loose et al., 2014; Lovely et al., 2015). If $k_{i c e}$ is negligible, then $k_{e f f}=(f) k$ (Takahashi et al., 2009; Evans et al., 2015; Butterworth and Miller, 2016). For Injection 2 , we determined $k$, the value for open water. We expect $k_{i c e}$ to be lower than $k$ because the ice acts as a physical barrier to gas exchange. The rate of gas molecular diffusion in water (Jähne et al., 1987a; King and Saltzman, 1995) is higher than gas diffusion through ice (Gosink et al., 1976; Ahn et al., 2008; Loose et al., 2011a; Lovely et al., 2015). However, the exact rate of gas diffusion through saltwater ice (and by extension the value of $\left.k_{i c e}\right)$ is not well constrained and likely varies based on the physical properties of the ice such as brine volume and temperature (Golden et al., 2007; Loose et al., 2011a; Zhou et al., 2013; Moreau et al., 2014; Lovely et al., 2015). An additional factor reducing air-water exchange in ice-covered waters is that ice significantly reduces fetch for wave generation and therefore wind-driven near-surface turbulence (Squire et al., 1995; Overeem et al., 2011). However, other processes may enhance near-surface turbulence in the presence of sea ice including convection associated with heat loss and brine rejection (Morison et al., 1992; Smith and Morison, 1993), boundary layer shear between ice and water (McPhee, 1992; Saucier et al., 2004), and wave interactions with drifting ice (Kohout and Meylan, 2008).

To evaluate these results within the framework of equation 5.5, we must estimate the fractional ice cover during Injection 1. During surveys by car along the shoreline of Whycocomagh Bay and satellite data indicated that the bay was nearly fully covered with a continuous sheet of ice from 31 March-10 April, except for an opening close to Little Narrows (Figure 5-2a-b). Beginning between 7 April and 12 April, a small region of water appeared to open up along the shoreline northwest of the site of Injection 1, however, by this time the tracer patch had moved eastward, close to Little Narrows and likely was not significantly affected by this open water (Figure 5-2c). We mapped out the location of the ice edge closest to Little Narrows by boat on 26 March, 7 April, and 12 April (Figure 5-1a). Using these surveys and shoreline data, we calculate that for the surface area of the bay between the injection site and Little Narrows, $f=0.01$ on 26 March, 0.05 on 7 April, and 0.08 on 12 April. The $f$ experienced by the tracer patch during Injection 1 is likely between 0.05-0.08 because the tracer flowed through the open water near Little Narrows between 6 April - 11 April. If $k$ for open water is the same for both injections, then our results are consistent with $k_{e f f}=(f) k$ with $f=0.064$, which is consistent with the fraction of open water we estimate for Injection 1 . Thus we conclude that $k_{i c e}$ was negligible, compared to $k$. For example, if $k_{i c e}$ was even $10 \%$ of $k$ for open water, then $k_{\text {eff }}$ for Injection 1 would have been $\sim 0.11 \mathrm{~m} \mathrm{~d}^{-1}$, more than double the observed value of $0.0457(0.0051) \mathrm{m} \mathrm{d}^{-1}$.

In calculating GOP and NOP by oxygen mass balance, we apply the tracer-based gas transfer velocities determined by dual tracer release throughout the time-series, rather than a traditional wind speed-based parameterization because the gas transfer velocities determined at the same time as the productivity estimates are most accurate, since there is no 
consensus on the best treatment of gas transfer in lakes and estuaries (Clark et al., 1995; Cole and Caraco, 1998; Crusius and Wanninkhof, 2003; Ho et al., 2011a), nor on the parameterization of gas transfer in the presence of ice (Else et al., 2011; Lovely et al., 2015; Butterworth and Miller, 2016). If bottom-derived turbulence (e.g., from tidal flow) is a significant contributor to air-water gas exchange, a parameterization based on wind speed alone may not be appropriate. Our method of calculating one average $k_{600}$ for each injection does not enable the calculation of wind speed-dependent parameterization for the gas transfer velocity.

Because the $k_{600}$ for Injection 1 and Injection 2 are so different, the treatment of the gas transfer velocity in between the two injections strongly affects our productivity estimates for this period. We use $k_{600}=0.0457(0.0051) \mathrm{m} \mathrm{d}^{-1}$ from the beginning of the time-series until midnight 16 April, the day when Figure 5-2d was collected. This image is the first satellite image showing substantial open water within Whycocomagh Bay, but the open water is primarily in the western half of the bay, far from Little Narrows. We use $k_{600}=$ $0.71(0.13) \mathrm{m} \mathrm{d}^{-1}$ from midnight 20 April until the end of the time-series on 28 April. Our surveys by boat on 19 April and 20 April indicated $<10 \%$ ice cover on these days and we collected the first tracer measurements following Injection 2 on 20 April 23:30. Between 16 April and 20 April, we apply a linear interpolation of the $k_{600}$ for Injection 1 and Injection 2 as a function of time. The gas transfer velocity is most uncertain during the period when the ice cover rapidly decreased because we do not have any measurements of gas transfer at intermediate ice cover. However, because the ice cover retreated rapidly, only four days of the productivity estimates (out of a 33-day time-series) are affected by the uncertainties in gas transfer at intermediate ice cover.

\section{Comparison with other estimates}

To compare the gas exchange estimates with other published studies, we use wind speed data measured at $10 \mathrm{~m}$ height $\left(u_{10}\right)$ at Eskasoni Reserve, $27 \mathrm{~km}$ northeast of Little Narrows (Figure 5-1) and archived by the Government of Canada. The archived data are two-minute averages measured once per hour. For Injection 2, between 20 April 23:00 and 23 April 11:00 the average wind speed was $2.6(1.4) \mathrm{m} \mathrm{s}^{-1}$, the median was $2.2 \mathrm{~m} \mathrm{~s}^{-1}$, and the calculated $k_{600}$ over this time period from dual tracer data is $0.71(0.13) \mathrm{m} \mathrm{d}^{-1}$. Cole and Caraco (1998) find $k_{600}=0.636(0.029) \mathrm{m} \mathrm{d}^{-1}$ (95\% confidence interval) and their estimate is independent of wind speed in a lake with daily wind speeds of $1.39(0.06) \mathrm{m} \mathrm{s}^{-1}$ (95\% confidence interval); this $k_{600}$ is consistent within uncertainty with our result. Standard open ocean parameterizations that use a quadratic dependence on wind speed predict $k_{600}$ $\simeq 0.5-0.6 \mathrm{~m} \mathrm{~d}^{-1}$ with uncertainties of $\sim 20 \%$ or $\sim 0.10 \mathrm{~m} \mathrm{~d}^{-1}$ (Ho et al., 2006; Sweeney et al., 2007; Wanninkhof, 2014). 
Crusius and Wanninkhof (2003) find that in a lake gas exchange can be estimated nearly equally well using three different parameterizations. Using their equations with our wind speed record, we calculate transfer velocities of $0.32-0.66 \mathrm{~m} \mathrm{~d}^{-1}$ and the velocity is most similar to our result when we use a constant gas transfer velocity $k_{600}=0.24 \mathrm{~m} \mathrm{~d}^{-1}$ for $u_{10}<3.7 \mathrm{~m} \mathrm{~s}^{-1}$ and $k_{600}=1.23 u_{10}-4.30$ for $u_{10} \geq 3.7 \mathrm{~m} \mathrm{~s}^{-1}$. However, in their study they parameterized the gas transfer using instantaneous (e.g. one-minute averaged) winds measured throughout the time-series, not once per hour, and emphasize the importance of including the variability in short-term winds when quantifying gas exchange at low wind speeds. If gas transfer velocity has a nonlinear dependence on wind speed then short-term measurements will more accurately represent the gas transfer than wind speeds averaged over longer periods (Livingstone and Imboden, 1993; Crusius and Wanninkhof, 2003). Since we only have two-minute averages measured once per hour (for a total of 60 measurements during Injection 2) the wind record we use may not fully represent the variability in winds during the period when we sampled Injection 2 .

A source of error in comparisons with published results is that the wind speed data come from a different location than the study area. Although Eskasoni Reserve is adjacent to the Bras d'Or Lake, the local topography and bathymetry is different near the reserve and in Whycocomagh Bay. Thus, it is likely that the wind speed and momentum stress at the airwater interface differs at Whycocomagh Bay compared to Eskasoni Reserve (Ortiz-Suslow et al., 2015).

The measurements are in agreement with other studies showing gas transfer velocity is significantly reduced under near-complete ice cover (Lovely et al., 2015; Butterworth and Miller, 2016) and contrast with studies showing enhanced gas transfer under $>85 \%$ ice cover (Fanning and Torres, 1991; Else et al., 2011). We find that $k_{e f f}=(f) k$ for $>90 \%$ ice cover but we cannot evaluate whether the same equation holds at intermediate ice cover because there was no injection at a lower fractional ice cover. In our study, the ice cover was near-continuous across the entire bay during Injection 1 and likely did not contain the polynyas and leads that are prevalent in the Arctic and Antarctic; differences in gas transfer behaviour are expected based on the nature of the ice pack.

\subsubsection{Gross oxygen production}

\section{Calculation}

The triple oxygen isotopic composition of $\mathrm{O}_{2}$ is an effective tracer of gross photosynthetic $\mathrm{O}_{2}$ production (Juranek and Quay, 2013). Due to reactions in the upper atmosphere that impart a small mass-independent isotopic signature on atmospheric oxygen, $\mathrm{O}_{2}$ derived from airwater exchange (from the atmosphere) has a unique triple isotopic signature compared to $\mathrm{O}_{2}$ generated by photosynthesis (from $\mathrm{H}_{2} \mathrm{O}$ ) and $\mathrm{O}_{2}$ consumed by respiration. To characterize 
the oxygen isotopic composition, we define

$$
\delta^{18} \mathrm{O}=X^{18} / X_{\text {std }}^{18}-1
$$

and express the $\delta^{18} \mathrm{O}$ in $\%$ by multiplying by 1000 . Here $X^{18}=r\left({ }^{18} \mathrm{O} /{ }^{16} \mathrm{O}\right)$ is the measured ratio and $X_{s t d}^{18}=r\left({ }^{18} \mathrm{O} /{ }^{16} \mathrm{O}\right)_{\text {std }}$ is the ratio of the isotopes in the standard. We calculate $\delta^{17} \mathrm{O}$ analogously. In this study, we use $\mathrm{O}_{2}$ in air, collected in Woods Hole, MA, as the standard for isotopic measurements of $\mathrm{O}_{2}$ and VSMOW (Vienna standard mean ocean water) as the standard for isotopic measurements of $\mathrm{H}_{2} \mathrm{O}$. For clarity, we distinguish between the isotopic composition of the two substrates $\left(\mathrm{O}_{2}\right.$ and $\left.\mathrm{H}_{2} \mathrm{O}\right)$ as $\delta^{18} \mathrm{O}-\mathrm{O}_{2}$ and $\delta^{18} \mathrm{O}-\mathrm{H}_{2} \mathrm{O}$.

We use the term ${ }^{17} \Delta$ to quantify the triple isotopic composition of dissolved $\mathrm{O}_{2}$

$$
{ }^{17} \Delta=\ln \left(\delta^{17} \mathrm{O}-\mathrm{O}_{2}+1\right)-\lambda \ln \left(\delta^{18} \mathrm{O}-\mathrm{O}_{2}+1\right) .
$$

We report ${ }^{17} \Delta$ with $\lambda=0.5179$, the ratio of the fractionation factors for respiratory $\mathrm{O}_{2}$ consumption in ${ }^{17} \mathrm{O}$ relative to ${ }^{18} \mathrm{O}$ (i.e. $\lambda={ }^{17} \epsilon /{ }^{18} \epsilon$, where $\epsilon$ is the isotopic fractionation of $\mathrm{O}_{2}$ due to respiratory consumption) (Luz and Barkan, 2005). We select this value for $\lambda$ so that ${ }^{17} \Delta$ is not altered by respiratory $\mathrm{O}_{2}$ consumption and reflects the proportion of $\mathrm{O}_{2}$ that is derived from photosynthesis relative to air-water gas exchange (Hendricks et al., 2005; Juranek and Quay, 2013; Nicholson et al., 2014).

Two key constraints in the calculation of GOP from measurements of the triple isotopic composition of $\mathrm{O}_{2}$ are the isotopic composition of $\mathrm{O}_{2}$ derived from air-water exchange, and the isotopic composition of photosynthetic $\mathrm{O}_{2}$. If the isotopic composition of one or both of these endmembers is defined incorrectly, it will cause systematic biases in the GOP estimate (Barkan and Luz, 2011; Kaiser, 2011; Luz and Barkan, 2011; Nicholson et al., 2011). The composition of photosynthetic $\mathrm{O}_{2}$ is dependent on the triple oxygen isotopic composition of $\mathrm{H}_{2} \mathrm{O}$, the substrate for photosynthetic $\mathrm{O}_{2}$, and the isotopic fractionation associated with photosynthetic $\mathrm{O}_{2}$ production. In oceanic studies, investigators typically assume, implicitly or explicitly, that $\mathrm{H}_{2} \mathrm{O}$ has an isotopic composition equivalent to VSMOW (Hamme et al., 2012; Nicholson et al., 2012; Manning et al., 2016b), which is a standard based on the average isotopic composition of deep ocean water $(500$ to $2000 \mathrm{~m}$ ) from a variety of locations (Epstein and Mayeda, 1953; Craig, 1961a). To determine the isotopic composition of photosynthetic $\mathrm{O}_{2}$, they apply a photosynthetic fractionation factor to VSMOW (Helman et al., 2005; Eisenstadt et al., 2010; Luz and Barkan, 2011). Using $\lambda=0.5179, \mathrm{O}_{2}$ that is purely biological in origin, derived from VSMOW, and generated by "average phytoplankton" (defined in Luz and Barkan (2011) Table 1) has ${ }^{17} \Delta \simeq 294$ per meg, and $\mathrm{O}_{2}$ derived from air-water equilibrium has ${ }^{17} \Delta \simeq 8$ per meg from near-freezing to $25{ }^{\circ} \mathrm{C}$ (Reuer et al. (2007); Stanley et al. (2010), and R.H.R. Stanley, unpublished data). We report ${ }^{17} \Delta$ in per meg (1 per meg $=0.001 \%$ ) due to the small range of values. 
Despite the typical assumption in these studies that the water isotopic composition is equivalent to VSMOW, surface ocean waters can differ from VSMOW by up to $7 \%$ for $\delta^{18} \mathrm{O}$ $\mathrm{H}_{2} \mathrm{O}$ on a regional basis, though are most commonly within $2 \%$ of VSMOW (LeGrande and Schmidt, 2006). Even more importantly for our study, in brackish and freshwater systems the isotopic composition of $\mathrm{H}_{2} \mathrm{O}$ can be substantially different from VSMOW (by up to $\sim 40$ $\%$ for $\delta^{18} \mathrm{O}-\mathrm{H}_{2} \mathrm{O}$ ) because the isotopic composition of meteoric (precipitation-derived) water has substantial regional and seasonal variability (Epstein and Mayeda, 1953; Craig, 1961b; Vachon et al., 2007; Feng et al., 2009; Jasechko et al., 2013).

In the Bras d'Or Lake, in order to accurately quantify the rate of gross oxygen production from $\delta^{17} \mathrm{O}-\mathrm{O}_{2}$ and $\delta^{18} \mathrm{O}-\mathrm{O}_{2}$ measurements, we must know or estimate $\delta^{17} \mathrm{O}-\mathrm{H}_{2} \mathrm{O}$ and $\delta^{18} \mathrm{O}-\mathrm{H}_{2} \mathrm{O}$ throughout the time-series. Because we did not measure the triple oxygen isotopic composition of $\mathrm{H}_{2} \mathrm{O}$, we use published measurements of $\delta^{18} \mathrm{O}-\mathrm{H}_{2} \mathrm{O}$ and published relationships between $\delta^{17} \mathrm{O}-\mathrm{H}_{2} \mathrm{O}$ and $\delta^{18} \mathrm{O}-\mathrm{H}_{2} \mathrm{O}$ to estimate the values of $\delta^{18} \mathrm{O}-\mathrm{H}_{2} \mathrm{O}$ and $\delta^{17} \mathrm{O}-\mathrm{H}_{2} \mathrm{O}$ during the time-series. Measurements of $\delta^{17} \mathrm{O}-\mathrm{H}_{2} \mathrm{O}$ are currently much more scarce than $\delta^{18} \mathrm{O}-\mathrm{H}_{2} \mathrm{O}$ and methods achieving similar precision to measurements of $\mathrm{O}_{2}$ dissolved in water have only been available for about 10 years (Barkan and Luz, 2005).

In this study, the majority of our near-surface measurements had a salinity ranging from 5-15 PSS. Thus, the waters represent a mixture of local seawater and local meteoric (precipitation-derived) water. A common approach in evaluate mixing between different water types is to plot salinity versus $\delta^{18} \mathrm{O}-\mathrm{H}_{2} \mathrm{O}$. In estuaries, both tend to behave conservatively and mix as a function of the two endmembers: seawater and meteoric water (Macdonald et al., 1995; Surge and Lohmann, 2002; Wankel et al., 2006). We estimate salinity and $\delta^{18} \mathrm{O}-\mathrm{H}_{2} \mathrm{O}$ for the two endmembers and then calculate $\delta^{18} \mathrm{O}-\mathrm{H}_{2} \mathrm{O}$ for each water sample collected during our time-series as a linear function of salinity.

For the seawater endmember, we use compilations of $\delta^{18} \mathrm{O}-\mathrm{H}_{2} \mathrm{O}$ and salinity (Schmidt, 1999; Bigg and Rohling, 2000) available from an online database (Schmidt et al., 1999). We included all near-surface samples ( $<5 \mathrm{~m}$ depth) between $44-48{ }^{\circ} \mathrm{N}$ and $58-64{ }^{\circ} \mathrm{W}$ in the database, for a total of 19 samples collected between 1973-1985. For these samples, the average $\delta^{18} \mathrm{O}-\mathrm{H}_{2} \mathrm{O}=-1.68(0.26) \%$ and salinity $=31.25(0.30)$ PSS.

For the meteoric endmember, we use an 8-year time-series of $\delta^{18} \mathrm{O}-\mathrm{H}_{2} \mathrm{O}$ measured in Truro, Nova Scotia (200 km southwest of our study area, $40 \mathrm{~m}$ elevation) and archived in the Global Network of Isotopes in Precipitation (GNIP) database (IAEA/WMO, 2016). The amount-weighted value of $\delta^{18} \mathrm{O}-\mathrm{H}_{2} \mathrm{O}$ over the time-series was -9.3(3.1) \%o versus VSMOW, using precipitation measurements from Truro NS over the same time period from the Government of Canada historical weather database (http://climate.weather.gc.ca). Also, Timsic and Patterson (2014) measured $\delta^{18} \mathrm{O}-\mathrm{H}_{2} \mathrm{O}=-8.8(0.1) \%$ on a water sample collected in July 2009 from the Skye River, within the Whycocomagh Bay watershed (Parker et al., 2007), consistent with the average Truro, NS value. 
Using the two endmembers, $\mathrm{S}=0 \mathrm{PSS}, \delta^{18} \mathrm{O}-\mathrm{H}_{2} \mathrm{O}=-9.3 \%$ (local meteoric water) and $\mathrm{S}=31.25$ PSS, $\delta^{18} \mathrm{O}-\mathrm{H}_{2} \mathrm{O}=-1.68 \%$ (local seawater), we calculate a relationship of $\delta^{18} \mathrm{O}$ $\mathrm{H}_{2} \mathrm{O}=0.2439 S-9.30$ (Figure 5-5). This linear relationship is consistent with published $\delta^{18} \mathrm{O}-\mathrm{H}_{2} \mathrm{O}$ measurements from within the estuary (Figure 5-5). Mucci and Page (1987) collected water samples from the Bras d'Or Lake in November 1985 and found a salinity of $\mathrm{S}=26.42(1.12)$ PSS and $\delta^{18} \mathrm{O}=-2.99(0.32) \%$ for samples at 17 different stations (albeit none within Whycocomagh Bay). Timsic and Patterson (2014) measured $\delta^{18} \mathrm{O}-\mathrm{H}_{2} \mathrm{O}=-$ $4.6(0.1) \%$ in a near-surface water sample from the southern shore of Whycocomagh Bay in July 2009, however, salinity was not measured on this sample, so it is not included in Figure 5-5 (S. Timsic, personal communication). The lower $\delta^{18} \mathrm{O}-\mathrm{H}_{2} \mathrm{O}$ value of this sample is likely reflective of the lower salinity within Whycocomagh Bay and near the shorelines (Yang et al., 2007), compared to samples collected by boat in other, more ocean-influenced regions of the Bras d'Or Lake by Mucci and Page (1987). Using a two-point linear regression for the local meteoric water and the Bras d'Or measurements from Mucci and Page (1987), for $\mathrm{S}=0-23$ PSS, we obtain $\delta^{18} \mathrm{O}-\mathrm{H}_{2} \mathrm{O}$ values within $0.12 \%$ of the regression based on local meteoric water and seawater. A regression using all three values gives results within $0.06 \%$ over the same salinity range. For simplicity, and because the results are very similar regardless of whether the Bras d'Or data is used in the regression, we apply the 2-endmember mixing equation for local seawater and meteoric water to estimate $\delta^{18} \mathrm{O}-\mathrm{H}_{2} \mathrm{O}$ throughout the time-series. Notably, VSMOW ( $\mathrm{S}=34.5 \mathrm{PSS}$ ) plots $0.9 \%$ above the mixing line, which demonstrates the importance of accurately defining both endmembers.

Then, for the two endmember values of $\delta^{18} \mathrm{O}-\mathrm{H}_{2} \mathrm{O}$, we calculate $\delta^{17} \mathrm{O}-\mathrm{H}_{2} \mathrm{O}$ using the following equation:

$$
{ }^{17} \mathrm{O} \text {-excess }=\ln \left(\delta^{17} \mathrm{O}-\mathrm{H}_{2} \mathrm{O}+1\right)-\lambda_{w} \ln \left(\delta^{18} \mathrm{O}-\mathrm{H}_{2} \mathrm{O}+1\right)
$$

with $\lambda_{w}=0.528$ and all isotopic compositions referenced to VSMOW. Note that equation 5.8 has a similar form to equation 5.7. The value of $\lambda_{w}=0.528$ is well established for meteoric waters and seawater (Meijer and Li, 1998; Landais et al., 2008; Luz and Barkan, 2010). Spatial variability in the ${ }^{17} \mathrm{O}$-excess of natural waters is less well understood due to the currently limited observations at sufficient accuracy to resolve the small excess (Luz and Barkan, 2010; Li et al., 2015). Relative humidity at the evaporation site is an important driver of the ${ }^{17} \mathrm{O}$-excess of meteoric waters (Angert et al., 2004; Barkan and Luz, 2007; Landais et al., 2008). To calculate the freshwater and seawater endmembers for $\delta^{17} \mathrm{O}-\mathrm{H}_{2} \mathrm{O}$ we use the average values of ${ }^{17} \mathrm{O}$-excess from Luz and Barkan (2010) of 33 per meg for meteoric water and -5 per meg for seawater. The endmembers are $\delta^{17} \mathrm{O}-\mathrm{H}_{2} \mathrm{O}=-4.888 \%$ \% and $-0.908 \%$ for meteoric water and seawater respectively, and the linear regression is $\delta^{17} \mathrm{O}-$ $\mathrm{H}_{2} \mathrm{O}=0.1274 S-4.89$. These $\delta$ values for $\mathrm{H}_{2} \mathrm{O}$, referenced to VSMOW, are subsequently 


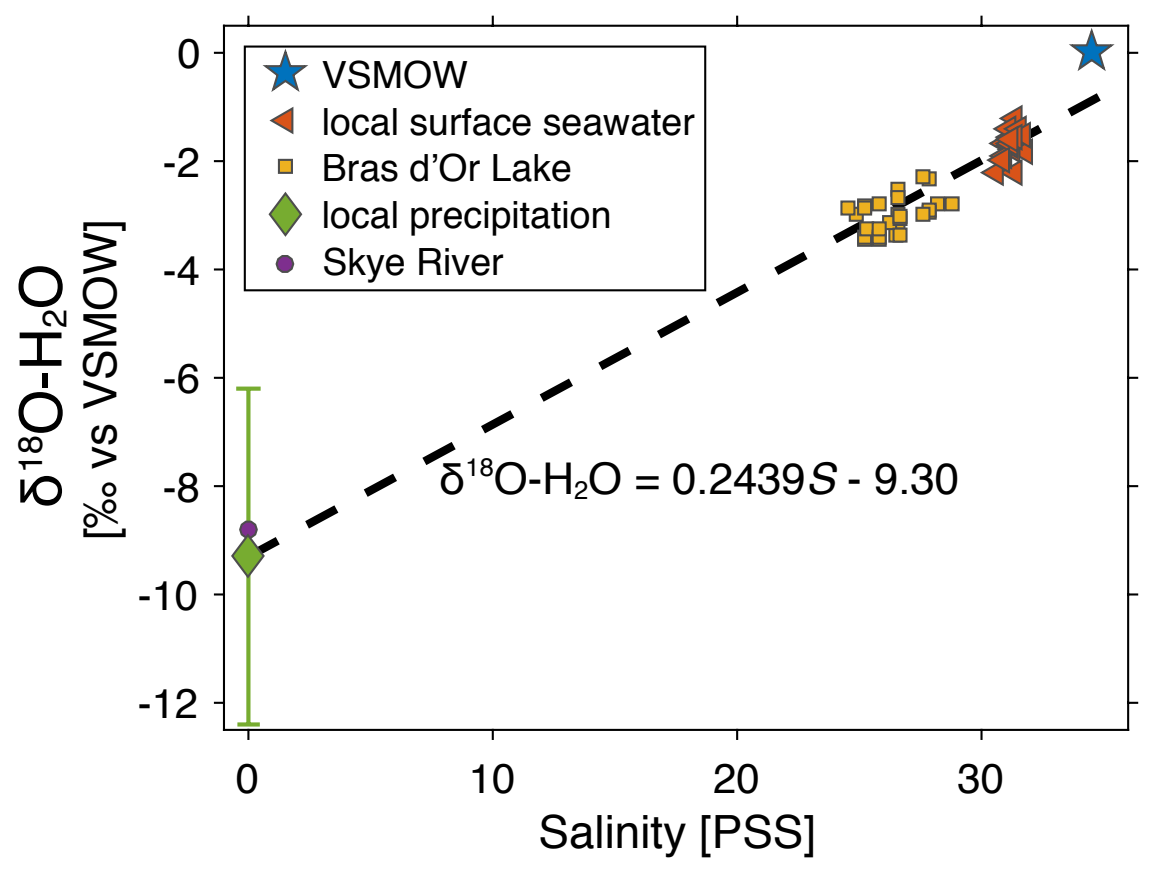

Figure 5-5: Measurements of $\delta^{18} \mathrm{O}-\mathrm{H}_{2} \mathrm{O}$ in local surface seawater, the Bras d'Or Lake, local precipitation, and Skye River (within the Whycocomagh Bay watershed). The regression is calculated using the local precipitation and seawater values. The local precipitation value is plotted as 9.3(3.1) \%o, with the uncertainty the standard deviation of the amount-weighted annual average. 
referenced to atmospheric $\mathrm{O}_{2}$ using results from Barkan and Luz (2011). In this study, the choice of $\delta^{18} \mathrm{O}-\mathrm{H}_{2} \mathrm{O}$ is more important than the ${ }^{17} \mathrm{O}$-excess because ${ }^{17} \mathrm{O}$-excess varies by less than $0.1 \%$ between samples whereas the freshwater $\delta^{18} \mathrm{O}-\mathrm{H}_{2} \mathrm{O}$ differs from VSMOW by $9.1 \%$. We discuss the sensitivity of the GOP calculations to the assumed ${ }^{17} \mathrm{O}$-excess and $\delta^{18} \mathrm{O}-\mathrm{H}_{2} \mathrm{O}$ below, and the effect of other processes on the isotopic composition of $\mathrm{H}_{2} \mathrm{O}$ in section 5.4.6.

We calculate GOP using equation S8 from Prokopenko et al. (2011)

$$
G O P=k_{\mathrm{O}_{2}}\left[\mathrm{O}_{2}\right]_{e q} \frac{\left[\frac{X^{17}-X_{e q}^{17}}{X^{17}}-\lambda \frac{X^{18}-X_{e q}^{18}}{X^{18}}\right]}{\left[\frac{X_{p}^{17}-X^{17}}{X^{17}}-\lambda \frac{X_{p}^{18}-X^{18}}{X^{18}}\right]}+\frac{h\left[\mathrm{O}_{2}\right] \frac{\partial^{17} \Delta}{\partial t}}{\left[\frac{X_{p}^{17}-X^{17}}{X^{17}}-\lambda \frac{X_{p}^{18}-X^{18}}{X^{18}}\right]} .
$$

Here $k_{\mathrm{O}_{2}}$ is the gas transfer velocity for $\mathrm{O}_{2}\left(\mathrm{~m} \mathrm{~d}^{-1}\right)$, $\left[\mathrm{O}_{2}\right]$ is the $\mathrm{O}_{2}$ concentration (mol $\left.\mathrm{m}^{-3}\right), h$ is the mixed layer depth $(\mathrm{m}), X^{17}=r\left({ }^{17} \mathrm{O} /{ }^{16} \mathrm{O}\right)$ and the subscripts $e q$ and $p$ refer to $\mathrm{O}_{2}$ at air-water equilibrium and produced by photosynthesis, respectively, and $\lambda=0.5179$ (equation 5.7). Oxygen terms without a subscript $\left(\left[\mathrm{O}_{2}\right], X^{17}\right.$, and $\left.{ }^{17} \Delta\right)$ are the measured mixed layer values. The first term on the right side of equation 5.9 is the steady state GOP term, and the second term is the non-steady state GOP term. If there is no change in ${ }^{17} \Delta$ with time (steady state with respect to ${ }^{17} \Delta$ ), then the second term on the right side of equation 5.9 equals zero.

We calculate $X_{e q}^{18}$ based on Benson and Krause (1980a, 1984), and $X_{e q}^{17}$ using ${ }^{17} \Delta_{e q}=8$ per meg (Reuer et al., 2007; Stanley et al., 2010), which is consistent with the daily measurements of distilled water equilibrated at room temperature that were analyzed along with the environmental samples (8.1 per meg with standard error of 1.6 per meg, $n=12$ ), as well as prior measurements of distilled water equilibrated at $<5{ }^{\circ} \mathrm{C}$ (R.H.R. Stanley, unpublished data). We calculate $X_{p}^{18}$ and $X_{p}^{17}$ using the salinity-dependent isotopic composition of $\mathrm{H}_{2} \mathrm{O}$ defined above, and isotopic fractionation factors for photosynthetic $\mathrm{O}_{2}$ with respect to $\mathrm{H}_{2} \mathrm{O}$ based on data in Luz and Barkan (2011) for average phytoplankton. The Matlab code used to calculate GOP and the triple oxygen isotopic composition of water (from two-endmember mixing of $\delta^{18} \mathrm{O}-\mathrm{H}_{2} \mathrm{O}$ and salinity) is available online (Manning and Howard, 2016).

We calculate gross oxygen production using samples collected at Little Narrows from 25 March-27 April (Figure 5-6). Visual inspection of the ${ }^{17} \Delta$ data indicated that ${ }^{17} \Delta$ changed during the time-series and therefore the calculation includes a non-steady state GOP term. The non-steady state term in equation 5.9 is $h\left[\mathrm{O}_{2}\right] \partial^{17} \Delta / \partial t$. To calculate the rate of change in ${ }^{17} \Delta$ with time, we first averaged the data into 24-hour bins (beginning and ending at 19:30, local sunset) to avoid over-weighting times when samples were collected at higher frequency. We calculated the average ${ }^{17} \Delta$ and sampling time for all samples collected each day. Next, we separated the data into two periods: one period began on 25 March and ended 19 April 07:30, and the second period covered the remainder of the time-series (ending 27 
April). We then applied a linear regression of ${ }^{17} \Delta$ versus time for the two time periods, which yielded a slope of 0.67 per meg $\mathrm{d}^{-1}\left(r^{2}=0.47\right)$ for the first period and -2.99 per meg $\mathrm{d}^{-1}\left(r^{2}=0.94\right)$ for the second period. The approximate timing for the change between periods was determined by visual inspection and then adjusted to maximize the $r^{2}$ and so that the equations of the two lines gave very similar ${ }^{17} \Delta$ values at 19 April 00:00 (within 1 per meg). We also tested splitting the period from 25 March-19 April into two separate regressions (or one period where ${ }^{17} \Delta$ increased and one period where it was constant) but found that this yielded much lower $\mathrm{r}^{2}$ values and a discontinuous ${ }^{17} \Delta$ record (different ${ }^{17} \Delta$ values at the end of one period and the start of another).

The other two variables in the non-steady state GOP term are the mixed layer depth (h) and $\left[\mathrm{O}_{2}\right]$. Because we do not have measurements of $\left[\mathrm{O}_{2}\right]$ for every sample, we estimate $\left[\mathrm{O}_{2}\right]$ as

$$
\left[\mathrm{O}_{2}\right] \simeq\left[\mathrm{O}_{2}\right]_{e q} \frac{\mathrm{O}_{2} / \mathrm{Ar}}{\mathrm{O}_{2} / \mathrm{Ar}_{e q}}
$$

This estimate assumes that $[\mathrm{Ar}]=[\mathrm{Ar}]_{e q}$. If $[\mathrm{Ar}]$ is, for example, $2 \%$ supersaturated then the estimated $\left[\mathrm{O}_{2}\right]$ and non-steady state GOP term will be $2 \%$ too high (Cassar et al., 2011).

Using equation 5.9, we calculate GOP for each sample, using an isotopic composition for $\mathrm{H}_{2} \mathrm{O}$ based on the salinity of the sample, and a mixed layer depth, rate of change in ${ }^{17} \Delta$ with time, and gas transfer velocity based on the sampling time, and then calculate the daily average GOP from all samples on a given day (beginning and ending at 19:30, local sunset). A few days had no measurements and the remainder of days had between 1-4 samples. The uncertainty in GOP on each day is calculated by propagating uncertainty in $k_{\mathrm{O}_{2}}(11 \%$ for Injection 1, $18 \%$ for Injection 2), uncertainty in the mixed layer depth (from 10-38 \%, 0.3 $\mathrm{m}$ ), uncertainty in the rate of change in ${ }^{17} \Delta$ with time (22\% and $9 \%$ where ${ }^{17} \Delta$ is increasing and decreasing, respectively), and uncertainty in the photosynthetic endmember (discussed below). Measurement uncertainty in the isotopic composition of $\mathrm{O}_{2}$ is excluded from the error calculation because it is a random error, rather than a systematic error (meaning that by taking many measurements of ${ }^{17} \Delta$ over several days, the measurements with high and low ${ }^{17} \Delta$ will average out) and because the measurement error is smaller than most other sources of error. All uncertainties are expressed as the standard deviation.

The isotopic composition of $\mathrm{H}_{2} \mathrm{O}$ one of the largest sources of error: if we shift the ${ }^{18} \mathrm{O}-\mathrm{H}_{2} \mathrm{O}$ endmembers for meteoric water and local seawater to the minimum values of $-12.4 \%$ and $-1.94 \%$ (one standard deviation below the mean value) and then re-calculate ${ }^{18} \mathrm{O}_{-} \mathrm{H}_{2} \mathrm{O}$ and ${ }^{17} \mathrm{O}-\mathrm{H}_{2} \mathrm{O}$ for each sample, GOP is on average $48 \%$ higher. If we shift the ${ }^{18} \mathrm{O}-\mathrm{H}_{2}$ Oendmembers for meteoric water and local seawater to the maximum values of -6.2 $\% 0$ and $1.42 \%$, respectively, GOP is on average $23 \%$ lower. The calculated GOP increases nonlinearly as the isotopic composition of photosynthetic $\mathrm{O}_{2}$ becomes more different from 

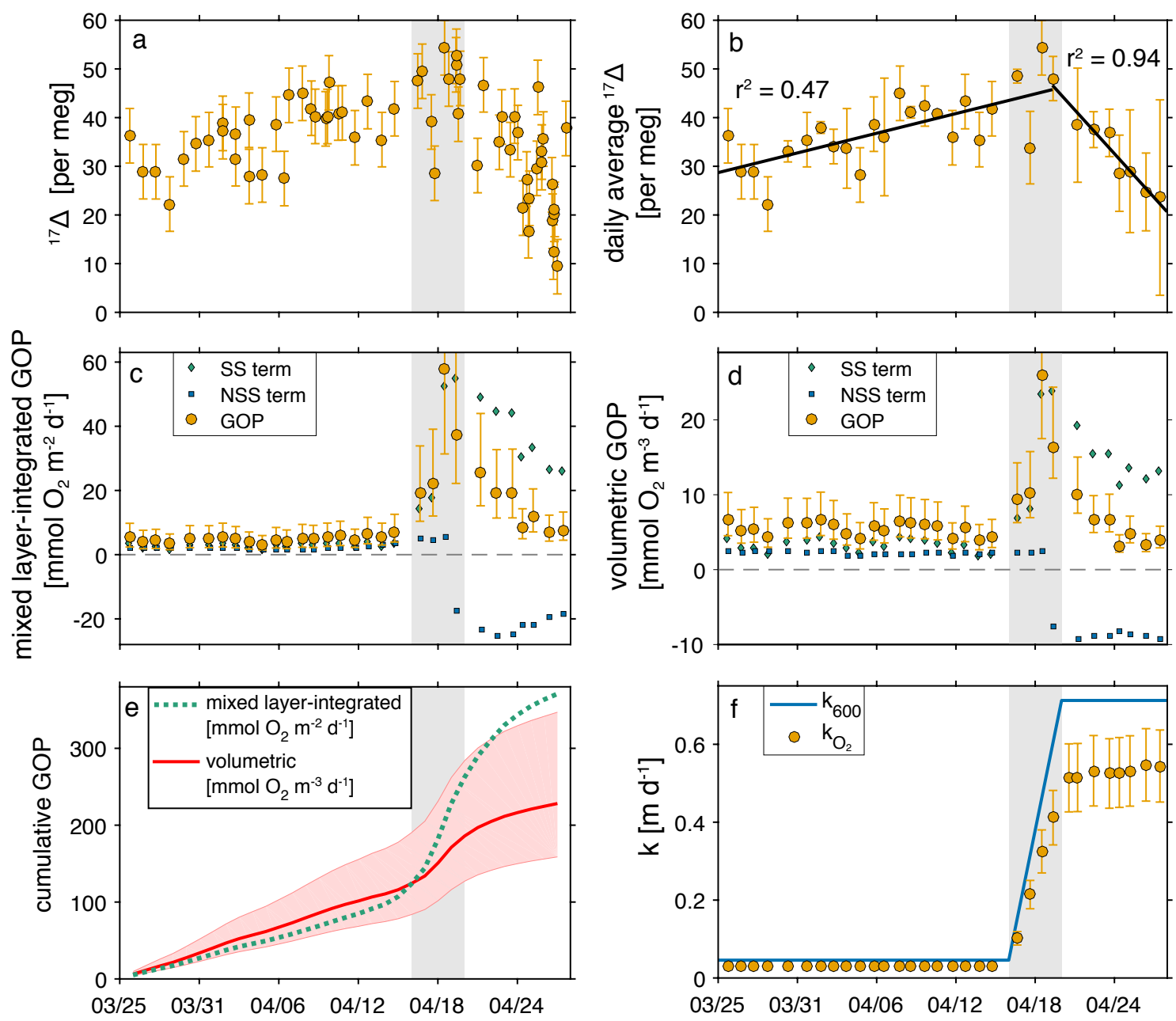

Figure 5-6: Gross oxygen production at Little Narrows and data used in the calculation. ${ }^{17} \Delta$ measurements from (a) all samples and (b) daily averaged values with the two linear regressions shown. Gross oxygen production in (c) mixed layer-integrated and (d) volumetric units. The green diamonds and blue squares show the values of the two terms in the GOP calculation (steady state, SS and non-steady state, NSS), and the yellow circles show the total GOP (the sum of the two terms). Error bars are only shown on the total GOP for clarity. The SS and NSS terms are slightly offset in time to make it easier to see both terms at the start of the time-series. (e) cumulative GOP in mixed layer-integrated and volumetric units; error (pink shaded range of values) is only shown for the volumetric GOP for clarity. (f) gas transfer velocity for a gas with a Schmidt number of 600 (blue line), and for $\mathrm{O}_{2}$ (yellow circles), which has a variable Schmidt number based on temperature and salinity). The grey shaded area in all plots is the period where gas exchange and the non-steady state GOP term are most uncertain. 
the isotopic composition of equilibrated $\mathrm{O}_{2}$. If we increase or decrease the ${ }^{17} \mathrm{O}$-excess of $\mathrm{H}_{2} \mathrm{O}$ by 20 per meg, GOP changes by an average of $10 \%$.

GOP calculated with the local isotopic composition of $\mathrm{H}_{2} \mathrm{O}$ is $46-97 \%$ higher (mean $74 \%)$ than GOP calculated assuming the water's isotopic composition is equivalent to VSMOW. Using the local isotopic composition of water instead of VSMOW is particularly important in this study because the system is not pure seawater. However, even in some oceanic regions such as the Arctic, the isotopic composition of $\mathrm{H}_{2} \mathrm{O}$ can be substantially different from VSMOW (LeGrande and Schmidt, 2006). The definition and importance of the photosynthetic endmember for GOP calculations in different environments warrants further review.

We calculate the mixed layer-integrated GOP $\left(\operatorname{mmol~} \mathrm{O}_{2} \mathrm{~m}^{-2} \mathrm{~d}^{-1}\right)$ and the volumetric GOP $\left(\mathrm{mmol} \mathrm{O} \mathrm{O}^{-3} \mathrm{~d}^{-1}\right)$, which is the mixed layer-integrated GOP divided by the mixed layer depth. For this time-series GOP is only calculated for the mixed layer because there are no $\mathrm{O}_{2}$ measurements below the mixed layer. The average errors in the daily GOP are ${ }_{-49}^{+77} \%$ and ${ }_{-31}^{+52} \%$ for the volumetric and mixed layer-integrated GOP, respectively. We also calculate the cumulative GOP (the sum of the daily GOP estimates, from the start of the time-series until a given day) which provides an estimate of the total amount of photosynthetic $\mathrm{O}_{2}$ produced by autotrophs during our time-series (Figure 5-6e) (Fassbender et al., 2016).

\section{Results and discussion}

GOP is most uncertain from 16 April through 19 April (grey shaded area in Figure 5-6). During this period, the ice cover was retreating and we do not have an estimate of $k_{\mathrm{O}_{2}}$ at intermediate ice cover, nor do we have estimates of ice cover on 17 April or 19 April, because clouds obscured the satellite images on those days. Additionally, the exact timing of the change in the sign of the non-steady state term is unclear. For example, in Figure 5-6b, 18 April is the final day where $\partial^{17} \Delta / \partial t$ is positive and the calculated GOP is $58 \mathrm{mmol} \mathrm{O}_{2}$ $\mathrm{m}^{-2} \mathrm{~d}^{-1}$. If 18 April is included in the period where ${ }^{17} \Delta$ is decreasing, the calculated GOP is $27 \mathrm{mmol} \mathrm{O}_{2} \mathrm{~m}^{-2} \mathrm{~d}^{-1}$ on 18 April. The concentration of photosynthetic $\mathrm{O}_{2}$ is highest during this time $\left({ }^{17} \Delta=48-54\right.$ per meg on 16 April, 18 April, and 19 April), despite the increasing gas transfer velocity. Therefore it is likely that GOP peaked at the end of the ice melt period and then declined to values similar to the beginning of the time-series, but the uncertainties in GOP from 16-19 April are large.

Overall, the rate of volumetric mixed layer GOP was relatively constant throughout the time-series (excluding the period from 16 April-20 April, when values are most uncertain) at $5.5\left({ }_{-1.7}^{+2.9}\right) \mathrm{mmol} \mathrm{O}_{2} \mathrm{~m}^{-3} \mathrm{~d}^{-1}$. The mixed layer-integrated GOP showed larger changes with time that are related to the influence of changes in the mixed layer depth on the non-steady state term. From 25 March through 8 April, mixed layer-integrated GOP was $4.6\left({ }_{-2.6}^{+3.7}\right)$ 
mmol $\mathrm{O}_{2} \mathrm{~m}^{-2} \mathrm{~d}^{-1}$. Beginning after 8 April, the mixed layer depth began to increase and the non-steady state calculation showed a substantial increase in mixed layer-integrated GOP, to $7.2\left({ }_{-3.3}^{+5.4}\right) \mathrm{mmol} \mathrm{O}_{2} \mathrm{~m}^{-3} \mathrm{~d}^{-1}$ on 14 April, a $56 \%$ increase. The non-steady state GOP term is multiplied by the mixed layer depth, and therefore it increases linearly as the mixed layer deepens, causing the total mixed layer-integrated GOP to decrease. However, the non-steady state GOP term is constant in volumetric units because the mixed layer depth cancels out of the equation. After the ice is gone, ${ }^{17} \Delta$ begins to decrease and so does the mixed layer-integrated GOP. On the last four days of the time-series, the mixed layerintegrated mixed layer GOP is $8.7\left({ }_{-6.3}^{+3.6}\right) \mathrm{mmol} \mathrm{O}_{2} \mathrm{~m}^{-2} \mathrm{~d}^{-1}, 89 \%$ higher than the average value prior to 9 April, but the mixed layer is also more than twice as deep at the end of the time-series compared to the beginning.

The influence of mixed layer depth on the GOP calculations is demonstrated with the cumulative GOP (Figure 5-6e). The volumetric GOP is roughly constant with time and therefore the cumulative volumetric GOP increases in an approximately linear fashion, except during the period from 16-19 April, where GOP is most uncertain. The mixed layerintegrated GOP is slightly lower than the volumetric GOP at the start of the time-series (because the mixed layer is slightly less than $1 \mathrm{~m}$ deep) and then as the mixed layer deepens, the cumulative mixed layer-integrated GOP increases rapidly. In the last few days of the time-series the mixed layer shoals and the rate of increase in cumulative mixed layerintegrated GOP is lower.

Overall, the result that volumetric GOP was similar at the beginning and end of the timeseries indicates that ice-free conditions are not a pre-requisite for phytoplankton growth in this system. Currently, ecosystem dynamics within and below freshwater ice are not well understood, and algal communities in ice formed at intermediate salinities (such as in the Bras d'Or Lake) are even less well studied (Salonen et al., 2009; Bertilsson et al., 2013; Hampton et al., 2015). Other investigators have shown that photosynthetic microbes can inhabit the interior, upper surface, and lower surface of ice, and tend to be most concentrated on the bottom surface (Welch et al., 1988; Cota et al., 1991; Frenette et al., 2008; Boetius et al., 2013). Traditionally, investigators have argued that ice-associated communities are most prevalent in ice formed from seawater; as salinity increases, the volume of unfrozen brines within the ice that the microbes can inhabit increases, and the bottom surface of the ice becomes more uneven, increasing bottom algal settlement efficiency (Legendre et al., 1981; Gosselin et al., 1986). However, more recently, investigators have also found algae growing within and on the bottom of freshwater ice in lakes and rivers, including locations in Canada such as the Great Lakes and the St. Lawrence River (Bondarenko et al., 2006; Frenette et al., 2008; Twiss et al., 2012; D'souza et al., 2013).

Phytoplankton can also grow in the water column beneath ice, especially thinner firstyear ice (Legendre et al., 1981; Mundy et al., 2009; Arrigo et al., 2012). Bare ice transmits 
more light to surface waters than snow-covered ice, and melt pond-covered ice transmits 4 times as much light as bare ice (Light et al., 2008; Arrigo et al., 2012; Light et al., 2015). First-year ice in the Arctic (0.5-1.5 m thick) transmits $\sim 47-75 \%$ of incident light through melt pond-covered ice and $\sim 13-25 \%$ of incident light through snow-free ice (Arrigo et al., 2012; Light et al., 2015). The ice in the Bras d'Or Lake near the site of Injection 1 was $~ 0.3$ $\mathrm{m}$ thick on 29 March and therefore likely similar or greater fractions of light were transmitted through the ice. Ice transmitting just $2 \%$ of surface irradiance may support high rates of photosynthetic activity, if the microbes are acclimated to lower light levels (Cota, 1985). We observed melt ponds on the Bras d'Or Lake during tracer injections on 31 March and frequently during visual surveys in April. The shallow mixed layer prior to ice melt $(\sim 0.8 \mathrm{~m}$ from the beginning of the time-series until 8 April) would have kept phytoplankton in the mixed layer close to the surface and therefore receiving light.

Our $\mathrm{O}_{2}$ mass balance techniques will record GOP by free-floating phytoplankton in the water column below the ice, as well as GOP by ice-associated phytoplankton if the $\mathrm{O}_{2}$ they produce diffuses into the water rather than into the ice surface. Bottom-associated algae likely release much of their $\mathrm{O}_{2}$ into the water column, especially for filamentous forms such as the diatoms frequently observed in Lake Erie and the Arctic (D'souza et al., 2013; Boetius et al., 2013).

\subsubsection{Net oxygen production and export efficiency}

\section{Calculation}

We quantify non-steady state NOP, incorporating the observed changes in $\mathrm{O}_{2} / \mathrm{Ar}$ during the time-series. We calculate NOP as

$$
N O P=k_{\mathrm{O}_{2}} \Delta\left(\mathrm{O}_{2} / \mathrm{Ar}\right)\left[\mathrm{O}_{2}\right]_{e q}+h \frac{\partial \Delta\left(\mathrm{O}_{2} / \mathrm{Ar}\right)}{\partial t}\left[\mathrm{O}_{2}\right]_{e q}
$$

where $k_{\mathrm{O}_{2}}$ is the real-time gas transfer velocity $\left(\mathrm{m} \mathrm{d}^{-1}\right),\left[\mathrm{O}_{2}\right]_{e q}$ is the equilibrium $\mathrm{O}_{2}$ concentration $\left(\mathrm{mol} \mathrm{m}^{-3}\right)$ and $h$ is the mixed layer depth $(\mathrm{m})$ (Hamme et al., 2012). The first term on the right side of equation 5.11 is the steady state NOP term, and the second term is the non-steady state NOP term, which is dependent on the rate of change in $\Delta\left(\mathrm{O}_{2} / \mathrm{Ar}\right)$ with time. To calculate the rate of change in $\Delta\left(\mathrm{O}_{2} / \mathrm{Ar}\right)$ with time, we resampled the data to a fixed $5 \mathrm{~s}$ interval (each scan of all masses took 5-6 s) and filled in gaps with a linear interpolation. Then we applied a third order lowpass Butterworth filter with a cutoff frequency of $0.3 \mathrm{~d}^{-1}$ to generate a smooth $\mathrm{O}_{2} /$ Ar record (Roberts and Roberts, 1978) (Figure 5-7). We selected the cutoff frequency to remove the short-term variability from tides and diel changes in photosynthesis and respiration, and to minimize the number of times the inflection of the curve changed while capturing the overall trends in $\mathrm{O}_{2} /$ Ar. Below we discuss the sensitivity of the calculated NOP to the choice of cutoff frequency. Finally, we calcu- 
lated the derivative of $\Delta\left(\mathrm{O}_{2} / \mathrm{Ar}\right)$ with respect to time using the filtered record. We applied the same filtering method to the in situ salinity and thermocouple temperature data and used the filtered data to calculate the $\left[\mathrm{O}_{2}\right]_{e q}$ and $k_{\mathrm{O}_{2}}$, to prevent short-term fluctuations in salinity and temperature from producing apparent changes in NOP. We calculate the daily NOP (from 19:30 to 19:29 local time) using the average $\partial \Delta\left(\mathrm{O}_{2} / \mathrm{Ar}\right) / \partial t$ based on the filtered record and the average $\Delta\left(\mathrm{O}_{2} / \mathrm{Ar}\right)$ (using the raw, unflitered data).

To calculate the uncertainty in NOP, uncertainty in $k_{\mathrm{O}_{2}}$ (11\% for Injection $1,18 \%$ for Injection 2), mixed layer depth $(0.3 \mathrm{~m}, 10-38 \%)$, and the non-steady state term are propagated. Uncertanty in the non-steady state term is based on the results using different filtering methods. For the cumulative NOP over the whole time-series, the non-steady state NOP changes by 3-13\% if we double or halve the cutoff frequency. A conservative $13 \%$ error associated with the cutoff frequency choice is included in the estimates of daily and cumulative NOP. Uncertainty in $\Delta\left(\mathrm{O}_{2} / \mathrm{Ar}\right)(<0.1 \%$, based on the mean offset between the EIMS and the discrete samples) has a negligible impact on NOP, relative to the other sources of error. The average errors in the daily NOP are $\pm 0.8 \mathrm{mmol} \mathrm{O}_{2} \mathrm{~m}^{-2} \mathrm{~d}^{-1}(34 \%)$ and \pm 0.3 mmol $\mathrm{O}_{2} \mathrm{~m}^{-3} \mathrm{~d}^{-1}$ (23\%) for the mixed layer-integrated and volumetric NOP, respectively. All uncertainties are the standard deviation.

Finally, we calculate the ratio of NOP to GOP for each daily estimate (the export efficiency). This ratio is similar to an f-ratio or an e-ratio (Dugdale and Goering, 1967; Laws et al., 2000) and provides information on the fraction of GOP that is available for export out of the mixed layer (Figure 5-7f). The uncertainties in the NOP/GOP ratio on each day are quite large. In a steady state NOP and GOP calculation, the gas transfer velocity $k_{\mathrm{O}_{2}}$ cancels out of the equation for the NOP/GOP ratio and therefore it is not a source of uncertainty; however, in the non-steady state term the $k_{\mathrm{O}_{2}}$ does not cancel out.

\section{NOP results and comparison of of NOP and GOP}

Based on the non-steady state NOP estimates, the ecosystem was on average net autotrophic as the ice was melting, from the beginning of the time-series through 15 April (mean volumetric NOP of 1.9(2.1) mmol $\mathrm{O}_{2} \mathrm{~m}^{-3} \mathrm{~d}^{-1}$, median $\left.2.5 \mathrm{mmol} \mathrm{O}_{2} \mathrm{~m}^{-3} \mathrm{~d}^{-1}\right)$. During the (nearly) ice-free period from 20 April through the end of the time-series, the community was on average net heterotrophic but with a smaller magnitude than during the start of the time-series (mean volumetric NOP of $-0.7(0.9) \mathrm{mmol} \mathrm{O}_{2} \mathrm{~m}^{-3} \mathrm{~d}^{-1}$, median $-0.7 \mathrm{mmol} \mathrm{O}_{2}$ $\mathrm{m}^{-3} \mathrm{~d}^{-1}$ ). When the bay was nearly full of ice cover (from the beginning of the time-series until 16 April), NOP was dominated by the non-steady state term and this term was positive except for between 31 March-3 April when it was negative but small in magnitude. As the ice cover decreased, the non-steady state term decreased and became negative on 18 April. From 18-22 April the steady state term is roughly equal in magnitude but opposite in sign to the non-steady state NOP term. The NOP is more strongly negative from $23-25$ 

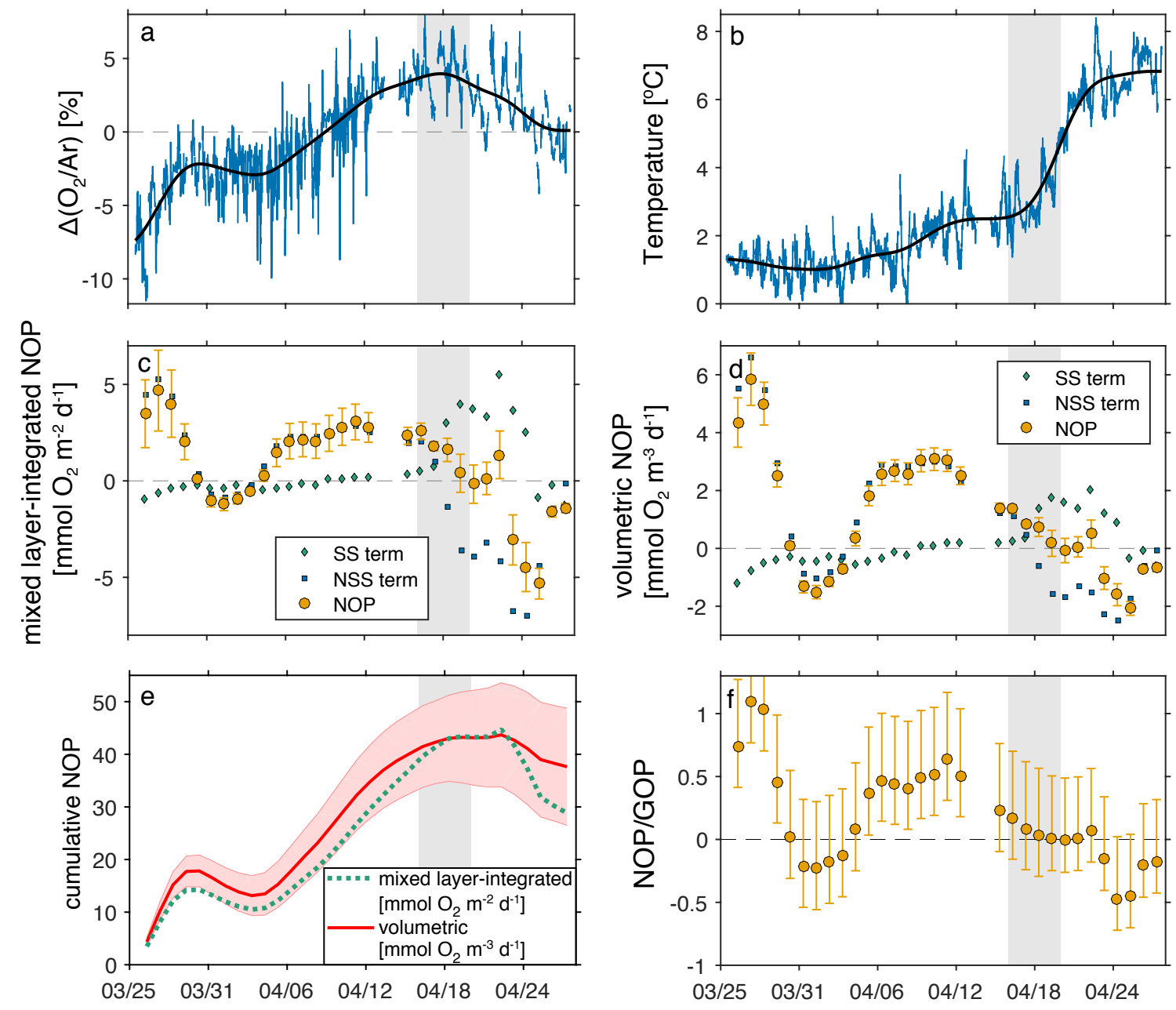

Figure 5-7: Net oxygen production at Little Narrows and data used in the calculation. (a) $\Delta\left(\mathrm{O}_{2} / \mathrm{Ar}\right)$ and (b) temperature measurements. The blue lines are the raw data and the black line is the filtered data. NOP in (c) mixed layer-integrated and (d) volumetric units. The yellow diamonds and pink triangles show the values of the two terms in the GOP calculation (steady state, SS and non-steady state, NSS). (e) cumulative NOP in mixed layer-integrated and volumetric units. (f) export efficiency ratio (NOP/GOP). The shaded grey area is the period where ice cover was decreasing rapidly and NOP is most uncertain. 
April (volumetric NOP of -1.6(0.5) $\mathrm{mmol} \mathrm{O}_{2} \mathrm{~m}^{-3} \mathrm{~d}^{-1}$ ) and then on the last two days of the time-series $\Delta\left(\mathrm{O}_{2} / \mathrm{Ar}\right)$ was close to 0 and so was the rate of change in $\Delta\left(\mathrm{O}_{2} / \mathrm{Ar}\right)$ with time (volumetric NOP of $-0.7 \mathrm{mmol} \mathrm{O}_{2} \mathrm{~m}^{-3} \mathrm{~d}^{-1}$ ). If the time-series were extended, we could determine whether the NOP value eventually stabilized near 0 following the dynamic ice melt period, or if it continued to oscillate between periods of net autotrophy and net heterotrophy.

Similarly to GOP, NOP is most uncertain between 16-19 April due to uncertainties in the fractional ice cover and the parameterization of gas exchange. However, the rate of change with time term is more certain for NOP during this period, compared to the same period in the GOP calculation, because the high-frequency measurements allowed us to calculate a smoothly varying non-steady state term, rather than an abrupt change in the non-steady state term based on a linear fit. The NOP appears to be decreasing during the period where the ice cover is changing most rapidly.

When summed over the entire time-series, the magnitude of the cumulative volumetric NOP (37.6(6.3) $\mathrm{mmol} \mathrm{O}_{2} \mathrm{~m}^{-3}$ ) is greater than the cumulative mixed layer-integrated NOP (28.8(4.4) $\mathrm{mmol} \mathrm{O}_{2} \mathrm{~m}^{-2}$ ). Volumetric NOP is negative in the second half of the time-series and the mixed layer is deepest during this period, causing the cumulative mixed layerintegrated NOP to decrease substantially. The cumulative NOP contrasts with cumulative GOP; the mixed layer-integrated GOP exceeds the volumetric GOP because GOP is always positive and the mixed layer was on average $>1 \mathrm{~m}$ deep.

The ratio of $\mathrm{NOP} / \mathrm{GOP}$ has large uncertainties but qualitatively follows the trends of NOP, since GOP was relatively constant throughout the time-series (except during and immediately following the ice cover reduction). At the start of the time-series there are two dates where NOP/GOP $>1$ which by definition is not possible. The high NOP/GOP values could be due to uncertainty in the isotopic composition of water, which enters into the GOP calculation but not the NOP calculation, and/or the non-steady state terms for GOP and NOP. Vertical mixing is another possible cause of the estimated NOP/GOP ratios exceeding 1; we cannot correct the calculations for vertical mixing because we do not have measurements below the mixed layer. The gradients in ${ }^{17} \Delta$ and $\Delta\left(\mathrm{O}_{2} / \mathrm{Ar}\right)$ are likely different with depth, leading to different magnitudes of the entrainment correction for NOP and GOP which could potentially lead to errors in the NOP/GOP ratio (section 5.4.6).

The different trends in GOP and NOP warrant some discussion. Volumetric GOP was relatively constant throughout the time-series except briefly at the end of the ice melt period where it was likely somewhat higher, whereas NOP decreased during and following the end of the ice melt period, and was negative from 23-27 April.

One possible explanation for the GOP results is that light may have limited productivity at the beginning of the time-series and nutrients may have limited productivity at the end of the time-series. In this case, the peak in GOP during the end of the melt period could 
indicate a reduction in light limitation causing enhanced growth rates, followed by the onset of nutrient limitation. Although nutrient concentrations have not been measured in the Bras d'Or Lake during winter or early spring, Strain and Yeats (2002) estimated a maximum wintertime $\mathrm{NO}_{3}{ }^{-}$concentration of $\sim 5 \mu \mathrm{M}$, based on fall nutrient measurements and assuming no primary production for 3 months. This $\mathrm{NO}_{3}{ }^{-}$concentration should be considered a maximum since the assumption of dormant phytoplankton in winter may not be correct (Hampton et al., 2015). Five $\mu \mathrm{M}$ of $\mathrm{NO}_{3}{ }^{-}$could support gross primary production of $33 \mathrm{mmol} \mathrm{C} \mathrm{m}{ }^{-3}$, using a Redfield ratio of 106:16 C:N (Redfield et al., 1963), and $46 \mathrm{mmol} \mathrm{O}_{2}$ $\mathrm{m}^{-3}$, using a ratio of 1.4:1 $\mathrm{O}_{2}$ :C for nitrate-based uptake (Laws, 1991). This concentration of $\mathrm{NO}_{3}{ }^{-}$would be consumed in 8 days at the average volumetric GOP of $5.5 \mathrm{mmol} \mathrm{O} \mathrm{m}^{-3}$ $\mathrm{d}^{-1}$, or just 3 days using the average GOP rate of $15(8) \mathrm{mmol} \mathrm{O}_{2} \mathrm{~m}^{-3} \mathrm{~d}^{-1}$ from $16-19$ April.

Another possible explanation for the GOP trends is that nutrients limited productivity throughout the time-series, causing GOP to be similar at the start and end of the timeseries. In this case, the likely increase in volumetric GOP during the most rapid reduction in ice cover could be due to changes in the water column that affected nutrient supply (e.g., nutrients were released from the melting ice, or the ice melt drove convection which entrained nutrients from deeper waters into the mixed layer).

The decrease in volumetric NOP must be due to an increase in respiration and recycling of organic carbon by heterotrophs (and possibly also autotrophs) within the mixed layer, since autotrophic production (GOP) stayed relatively constant. One possible explanation of the NOP trends is that zooplankton growth rates increased as the ice was melting and water warmed, leading to an increase in respiration at the same time that GOP was decreasing due to nutrient limitation. Zooplankton growth rates may increase more rapidly than phytoplankton growth rates in response to increasing temperatures (Rose and Caron, 2007). The water temperature in the system rapidly increased between 12-24 April from $\sim 2$ to 7 ${ }^{\circ} \mathrm{C}$, as the ice cover retreated and the surface waters absorbed more heat and therefore it is likely that growth rates also increased over this period (Figure 5-7b).

A second possible explanation for the decreasing NOP is that ice melt (and perhaps riverine inflow) increased the organic carbon concentrations within the water column and therefore increased grazing rates by heterotrophs. Zooplankton are thought to graze primarily on free-floating phytoplankton and algae that are released from the ice, rather than algae attached to the ice surface, and organic carbon sedimentation events associated with ice melt have been observed in other environments (Michel et al., 2002; Boetius et al., 2013). Concentrations of organic carbon in ice vary widely. Boetius et al. (2013) measured average ice algal deposition to sediments of $750 \mathrm{mmol} \mathrm{C} \mathrm{m}^{-2}$ during summer in the Arctic. If a similar amount of ice algae were released from the ice in the Bras d'Or Lake, it would be more than sufficient to support the observed increase in respiration (decrease in NOP) of up 
to $\sim 7 \mathrm{mmol} \mathrm{O} 2 \mathrm{~m}^{-2} \mathrm{~d}^{-1}\left(5-6 \mathrm{mmol} \mathrm{C} \mathrm{m} \mathrm{m}^{-2} \mathrm{~d}^{-1}\right.$, using a C:O 2 ratio of 1.1-1.4 (Laws, 1991; Bender et al., 1999)) at the end of the time-series. Additionally, river flow on Cape Breton Island typically peaks in April or May, and river flows in April are 40-100 \% higher than flows in March and 50-150\% greater than the average monthly flow. However, dissolved organic carbon concentrations of rivers in Cape Breton during early spring have not been measured and the bioavailability of terrigenous organic carbon to aquatic microbes is not well known (Wiegner et al., 2006; Caissie and Robichaud, 2009; Guillemette and del Giorgio, 2011).

However, for both of these potential explanations of the NOP results, the increased respiration rates should also increase the nutrient supply available to autotrophs which would counteract nutrient limitation of GOP. Therefore, we might expect the observed increase in respiration (decrease in NOP) to favor an increase in GOP because the organic matter is being more efficiently recycled and therefore available to support more autotrophic production within the mixed layer. It is possible that the increase in mixed layer depth between 16-23 April diluted the autotrophic biomass and caused volumetric GOP to decrease.

Additionally, the uncertainties in GOP are relatively large, averaging ${ }_{-31}^{+52} \%$ for the volumetric GOP throughout the time-series, and therefore it is possible that there were moderate differences in GOP between the beginning and end of the time-series that are not apparent from our methods. For example, a systematic error in the gas transfer velocity at the end of the time-series (the Injection 2 result) would cause us to systematically overestimate or underestimate GOP at the end of the time series, if our calculated $k_{\mathrm{O}_{2}}$ were too high or too low, respectively. We discuss the potential impacts of other physical processes on our GOP and NOP estimates below (section 5.4.6).

\subsubsection{Comparison to other productivity estimates}

To our knowledge, our data are the only published estimates of NOP and GOP in the Bras d'Or Lake. Geen (1965) measured primary production by ${ }^{14} \mathrm{C}$ uptake in the Bras d'Or Lake during summer 1962-1964 and found an average uptake rate at $5 \mathrm{~m}$ depth, the depth of maximum photosynthesis, of $\sim 4 \mathrm{mmol} \mathrm{C} \mathrm{m}{ }^{-3} \mathrm{~d}^{-1}$ (50 $\mathrm{mg} \mathrm{C} \mathrm{m}^{-3} \mathrm{~d}^{-1}$ ) (Geen and Hargrave, 1966). These rates are based on 6 -h daytime incubations and ignore nighttime respiratory loss of ${ }^{14} \mathrm{C}$, and therefore are likely measuring net primary production (NPP, gross primary production minus autotrophic respiration) or something between NPP and gross primary production (GPP) (Bender et al., 1999; Marra, 2002, 2009; Quay et al., 2010). If we assume a $\mathrm{C}: \mathrm{O}_{2}$ ratio of 1.1-1.4 (Laws, 1991) we calculate an equivalent $\mathrm{O}_{2}$ production of 4.4-5.6 mmol $\mathrm{O}_{2} \mathrm{~m}^{-3} \mathrm{~d}^{-1}$ from the ${ }^{14} \mathrm{C}$ incubations. Thus, the ${ }^{14} \mathrm{C}-\mathrm{PP}$ is between the average NOP and GOP values, as expected.

Hargrave and Geen (1970) found, based on incubation,s that zooplankton grazing was sufficient to consume all of the daily primary production, indicating the estuary ecosystem 
metabolism may be close to balanced (NOP $\sim 0$ ). We obtained a more dynamic record of NOP, with an average volumetric rate of 1.1(2.0) $\mathrm{mmol} \mathrm{O}_{2} \mathrm{~m}^{-3} \mathrm{~d}^{-1}$ over the entire time-series.

Comparisons with in situ gas tracer-based estimates in other environments are challenging because mixed layer-integrated rates are most commonly calculated, and the mixed layer in many other systems is much deeper than 0.8-3 m. In the Beaufort Gyre (Arctic Ocean) (Stanley et al., 2015) estimate a steady state GOP of 16(5) and 38(3) $\mathrm{mmol} \mathrm{O}_{2} \mathrm{~m}^{-2} \mathrm{~d}^{-1}$ in summer 2011 (higher ice cover) and 2012 (lower ice cover), respectively and NOP of $3 \mathrm{mmol}$ $\mathrm{O}_{2} \mathrm{~m}^{-2} \mathrm{~d}^{-1}$ in both summers. Mixed layer depths were $\sim 10 \mathrm{~m}$. Mixed layer-integrated GOP increases as the mixed layer depth increases, but trends in NOP are less clear. In this study we found that cumulative volumetric and cumulative mixed layer-integrated NOP were similar when calculated over the entire time-series, but the result might be different if we integrated to $10 \mathrm{~m}$.

\subsubsection{Effect of physical processes on productivity estimates}

There are a number of additional environmental processes that may affect the $\mathrm{O}_{2}$ isotope and $\mathrm{O}_{2} / \mathrm{Ar}$ mass balance in the mixed layer but cannot be directly quantified from the time-series. However, in some cases we can determine whether these processes would tend to increase or decrease our NOP and GOP estimates.

The isotopic composition of freshwater within the bay may have varied during our timeseries, which would impact the GOP estimates. Using model results from Gurbutt and Petrie (1995) we calculate that the residence time of water in Whycocomagh Bay is $\sim 0.7 \mathrm{y}$ for surface waters $(0-10 \mathrm{~m})$ with respect to freshwater input and $\sim 2 \mathrm{y}$ for deep waters $(10 \mathrm{~m}$ to bottom) with respect to exchange with the surface waters. Thus we expect the isotopic composition of the water in the mixed layer of Whycocomagh Bay to reflect some average over multiple months. For example, if a substantial portion of the meltwater entering the estuary is derived from snow rather than from ice that freezes from water within the bay, its isotopic composition will be more reflective of seasonal precipitation. If we calculate an amount-weighted $\delta^{18} \mathrm{O}-\mathrm{H}_{2} \mathrm{O}$ for meteoric water at Truro, NS, using only the months when ice was present in Whycocomagh Bay in 2013 (January-April), $\delta^{18} \mathrm{O}-\mathrm{H}_{2} \mathrm{O}=-11.0(3.6) \%$ versus VSMOW, which is within the uncertainty of our annually-averaged value $(-9.3(3.1)$ $\%$ ). In general, a lower value of $\delta^{18} \mathrm{O}-\mathrm{H}_{2} \mathrm{O}$ will increase GOP.

The freezing and melting of ice in saline waters will generate a non-linear salinity- $\delta^{18} \mathrm{O}$ $\mathrm{H}_{2} \mathrm{O}$ relationship because the $\delta^{18} \mathrm{O}-\mathrm{H}_{2} \mathrm{O}$ value of sea ice is similar to the water from which it formed (within $\sim 2-3 \%$ ) (Tan and Strain, 1980; Macdonald et al., 1995, 1999), but the salinity is substantially lower due to brine rejection (O'Neil, 1968; Weeks and Ackley, 1986). We are not able to accurately quantify the triple oxygen isotopic composition of $\mathrm{H}_{2} \mathrm{O}$ in the ice and in water, nor can we quantify the timing and volume of ice freezing and melt 
within Whycocomagh Bay (although we know when the ice cover decreased most rapidly, the ice volume was decreasing throughout our time-series). The volume contribution and isotopic composition of other sources of water inputs (e.g., riverine input and melting snow) is another source of uncertainty in the calculations.

In addition to affecting the isotopic composition of $\mathrm{H}_{2} \mathrm{O}$, ice melt and riverine inflow may affect the NOP and GOP calculations in other ways. If the ice melted at the upper surface (in contact with the atmosphere) and then drained through brine channels in the ice, it likely had an isotopic composition and gas ratio similar to air-equilibrated water $\left({ }^{17} \Delta\right.$ $\simeq 8$ per meg and $\left.\Delta\left(\mathrm{O}_{2} / \mathrm{Ar}\right) \simeq 0 \%\right)$. Thus, water in melt ponds that was added to the water column would tend to decrease GOP, as ${ }^{17} \Delta$ always exceeded 8 per meg in the mixed layer (Figure 5-6 a) and either increase or decrease NOP, since $\Delta\left(\mathrm{O}_{2} / \mathrm{Ar}\right)$ transitioned from negative to positive as the ice was melting. If the ice melted at the bottom (in contact with the water) its effect on NOP and GOP estimates is less clear. During sea ice formation, approximately $40-55 \%$ of the $\mathrm{O}_{2}$ and Ar originally dissolved in the water is retained in the ice matrix (i.e. within the ice itself, in gas bubbles, or in brine pockets) and the remainder is excluded, generating supersaturations of the gases in the water below the ice (Top et al., 1988; Hood et al., 1998; Loose et al., 2009a). Photosynthesis and respiration both occur in sea ice (Loose et al., 2011b; Zhou et al., 2014) and will change the $\mathrm{O}_{2} / \mathrm{Ar}$ and ${ }^{17} \Delta$ signatures within the ice and it is difficult to predict what proportions of the $\mathrm{O}_{2}$ within brine pockets in the ice remained within the brines, migrated into the water column, or migrated into the atmosphere prior to the complete melting of the ice. We measured an ice thickness of $\sim 0.3 \mathrm{~m}$ near the injection site on $29 \mathrm{March}$, and thus if the mixed layer depth after ice melt was $2.5 \mathrm{~m}$ deep, the ice could contribute $\sim 11 \%$ of the mixed layer volume, or $\sim 5 \%$ of the mixed layer $\mathrm{O}_{2}$ (assuming $\left[\mathrm{O}_{2}\right]$ in ice is $\sim 45 \%$ of the equilibrium $\left[\mathrm{O}_{2}\right]$ in water). Thus bottom ice melt would likely be a minor influence on the oxygen mass balance. We observed bare ice and melt ponds at the surface of the ice (Figures D-3-D-5) and the water temperature throughout our time-series was above freezing which would stimulate bottom melt. Therefore, both surface and bottom melt likely occurred during the time-series. The volume, $\mathrm{O}_{2}$ concentration and isotopic composition of runoff and river water during our time-series is also poorly constrained and thus these water sources are another uncertainty in our NOP and GOP calculations.

It is likely that GOP occurred below the mixed layer but was not quantified by our methods because we only had measurements within the mixed layer. In open water, the Secchi depth at the ice edge on 26 March (just west of Little Narrows) was $1.9 \mathrm{~m}$, yielding a euphotic zone depth of $\sim 5 \mathrm{~m}$ (defined as the depth where $1 \%$ of surface photosynthetically active radiation penetrates) and the Secchi depth at Little Narrows on 7 April was $\sim 4.5 \mathrm{~m}$ giving an approximate euphotic zone depth of $12 \mathrm{~m}$ (Idso and Gilbert, 1974). Mixed layer depths during our time-series ranged from 0.8-3.0 m. Even when $\mathrm{O}_{2}$ measurements below 
the euphotic zone are available, it is challenging to quantify this subsurface productivity because the biological $\mathrm{O}_{2}$ fluxes below the mixed layer are small and the physical fluxes are large and highly uncertain, as they are driven by lateral and vertical mixing rather than air-water gas exchange (Giesbrecht et al., 2012; Munro et al., 2013; Manning et al., 2016b).

We also cannot correct our results for the effect of entrainment of deeper waters into the mixed layer (Hendricks et al., 2004; Munro et al., 2013; Wurgaft et al., 2013). We were not able to sample below the mixed layer but we hypothesize that ${ }^{17} \Delta$ and $\mathrm{O}_{2} / \mathrm{Ar}$ both likely decreased below the mixed layer when the ice was melting and the mixed layer was deepening. Therefore, entrainment of these waters into the mixed layer would tend to decrease NOP and GOP estimates. Respiration increases with depth, causing $\left[\mathrm{O}_{2}\right]$ and $\Delta\left(\mathrm{O}_{2} / \mathrm{Ar}\right)$ to decrease with depth in oceanic systems (Spitzer and Jenkins, 1989; Emerson et al., 1991). In some oceanic regions, photosynthesis below the mixed layer generates excess ${ }^{17} \Delta$ (because there is no process decreasing ${ }^{17} \Delta$ below the mixed layer), which can then be entrained into the euphotic zone, where ${ }^{17} \Delta$ is lower because some of the photosynthetic $\mathrm{O}_{2}$ is ventilated to the atmosphere (Hendricks et al., 2004; Sarma et al., 2005; Juranek and Quay, 2013). In this study, the gas transfer velocity out of the mixed layer was extremely low up until 16 April, and therefore photosynthetic $\mathrm{O}_{2}$ (i.e., ${ }^{17} \Delta$ ) would also accumulate in the mixed layer, likely at a greater rate than below the mixed layer due to photosynthesis rates being higher closer to the surface. Once the gas transfer velocity increased to the open water value (20 April), it is not clear whether we would expect to observe an excess of ${ }^{17} \Delta$ below the mixed layer, and therefore whether entrainment would increase or decrease GOP.

Another source of error in our interpretation of the $\mathrm{O}_{2}$ data is that we must assume that spatial variability in $\mathrm{O}_{2}$ has a negligible effect on our calculations. We interpret all changes in $\mathrm{O}_{2}$ assuming that we are measuring the same water mass; this is an oversimplification because the bay is within an estuary that experiences tidal flows. One reason we chose to install the instruments in one location rather than conducting spatial surveys is that during a pilot experiment in 2011 we found that spatial variability in $\mathrm{O}_{2}$ within the Bras d'Or Lake was relatively low. We hypothesized that obtaining a longer near-continuous time-series of $\mathrm{O}_{2} / \mathrm{Ar}$ (at all times of day) throughout the ice melt period would be more scientifically informative than periodic ship surveys which could only be conducted during daytime and only over a restricted area until the ice was fully melted.

\subsection{Conclusions}

Using the dual tracer $\left({ }^{3} \mathrm{He} / \mathrm{SF}_{6}\right)$ technique in the Bras d'Or Lake, we found that at $>90 \%$ ice cover, the gas transfer velocity was $6 \%$ of the open water gas transfer velocity. This result indicates that $k_{i c e}$ is negligible.

The volumetric GOP was similar at the beginning and end of the time-series, when the 
basin was full of ice and when it was ice free, but likely increased in the middle of the time-series as the last of the ice melted. Volumetric NOP was more variable with time; Whycocomagh Bay was on average net autotrophic (NOP $>0$ ) while the ice was melting, and net heterotrophic $(\mathrm{NOP}<0)$ but with a smaller magnitude after the bay was ice-free. These results indicate that an algal bloom (increasing NOP) can occur in an ice-covered estuary, similar to observations in the Great Lakes, Arctic, and Antarctic. The decrease in NOP may be due to the onset of nutrient limitation and/or an increase in heterotrophic respiration of organic carbon released from the ice and possibly also from rivers.

Obtaining a time-series of $\mathrm{O}_{2}$ data and obtaining simultaneous gas transfer velocity measurements were both critical for quantifying productivity. The non-steady state term was a significant contributor to NOP and GOP throughout the time-series, and time-series measurements are needed to quantify the non-steady state $\mathrm{O}_{2}$ flux. Additionally, because the gas transfer velocity was $\sim 16$ times higher at the end of the time-series than at the beginning and the values of ${ }^{17} \Delta$ and $\Delta\left(\mathrm{O}_{2} / \mathrm{Ar}\right)$ changed with time, the relative importance of the steady state term versus the non-steady state term changed substantially during our time-series.

\subsection{Acknowledgments}

We thank Zoe Sandwith (WHOI), Ann Lovely (University of Rhode Island), and Dave Woodland (Cape Breton University) for field and laboratory assistance. We also thank the staff of the Little Narrows Ferry Terminal for providing facilities. RHR Stanley and B Loose received funding from the WHOI Arctic Research Initiative, and CC Manning received graduate scholarships from NSERC and CMOS. 


\section{Chapter 6}

\section{Conclusions and future directions}




\subsection{Thesis summary}

For this thesis, I developed a new noble gas analysis system and applied $\mathrm{O}_{2}$ and inert gas tracers to study chemical, biological, and physical processes in coastal waters. In Chapter 2, I described a new field-deployable system for measurement of four noble gases in water and used the system to obtain high-frequency noble gas measurements in Waquoit Bay, MA. In Chapters 3-4, I collected a six-day time series of dissolved $\mathrm{O}_{2}$ and five stable noble gases. I evaluated the performance of four published gas exchange parameterizations in simulating the noble gas data and showed that I could correct the gas mass balances for vertical mixing by using vertical microstructure profiles obtained throughout the cruise. I compared gas tracer-based measurements of net community production and gross primary production with concurrent incubation-based $\left({ }^{14} \mathrm{C},{ }^{15} \mathrm{NO}_{3}{ }^{-}\right.$, and ${ }^{15} \mathrm{NH}_{4}{ }^{+}$uptake $)$and sediment trap-based estimates of productivity. To my knowledge, this study is the first published data set where all of these productivity techniques have been used simultaneously. In Chapter 5, I quantified productivity and air-water gas exchange by dual tracer release during seasonal ice melt in a Canadian estuary. I also demonstrated the importance of using the local isotopic composition of $\mathrm{H}_{2} \mathrm{O}$ in gross primary production (GPP) calculations and presented a new method of calculating the triple oxygen isotopic composition of water in estuaries using two salinity and $\delta^{18} \mathrm{O}-\mathrm{H}_{2} \mathrm{O}$ endmembers. This study is the first field-based experiment where the dual tracer technique is used to quantify the gas transfer velocity in the presence of ice. Together, the research in my thesis demonstrates that $\mathrm{O}_{2}$ and inert gases are effective tracers of a variety of processes in coastal waters. Below, I outline a few of the many areas for future research that would expand upon the results in my thesis, and I summarize the broader implications of my work.

\subsection{Future directions}

\subsubsection{Applications and modifications to the gas equilibration mass spec- trometer}

There are numerous applications for the portable noble gas analyzer (gas equilibration mass spectrometer, GEMS) described in Chapter 2. The system is ideal for laboratory experiments where high-frequency sampling is beneficial, for example in studies of gas exchange and gas partitioning during ice formation and melt (Loose et al., 2009a; Lovely et al., 2015), and studies of air-water gas exchange using wind-wave tanks or bubble plume generators (Asher et al., 1996; Callaghan et al., 2014; Krall and Jähne, 2014; Mesarchaki et al., 2015b; Nagel et al., 2015). It could be used for continuous, high-frequency monitoring of surface waters in order to improve understanding of physically-driven gas fluxes, and be used alongside instruments measuring the fluxes of biologically-active gases (Cassar et al., 2009; 
Rafelski et al., 2015). In recent years, numerous sensors for measuring dissolved gases have become available, including gas tension devices that measure the total gas pressure, and can be used to derive $\mathrm{N}_{2}$ concentration when paired with an $\mathrm{O}_{2}$ sensor (McNeil et al., 2005, 2006), and portable analyzers for individual dissolved gases including $\mathrm{CO}_{2}, \mathrm{CH}_{4}$, and $\mathrm{N}_{2} \mathrm{O}$ (Gonzalez-Valencia et al., 2014; Grefe and Kaiser, 2014; Bastviken et al., 2015). Simultaneous measurements of noble gases and bioactive gases would assist in separately quantifying the physical and biological fluxes for bioactive gases. It may be possible to determine concentrations of all four noble gases using the GEMS (in addition to their ratios) if the system is used alongside a second mass spectrometer measuring $\mathrm{O}_{2} / \mathrm{Ar}$ and a calibrated $\mathrm{O}_{2}$ sensor (Kana et al., 1994; Cassar et al., 2009; Eveleth et al., 2014).

Modifications could enable the system to measure $\mathrm{He}$, a gas that is less soluble than Ne and therefore more sensitive to differences in the treatment of bubble-mediated exchange in gas exchange parameterizations (Machler et al., 2012; Visser et al., 2013), and/or to measure discrete samples rather than a continuous water flow (Kana et al., 1994; Visser et al., 2013). Discrete samples would reduce the water volume requirements for analysis and enable samples from a wider range of locations to be analyzed, such as locations where power and shelter for the instrument is unavailable. Furthermore, analysis of discrete samples would likely make it possible to determine individual gas concentrations rather than their ratios (Machler et al., 2012; Visser et al., 2013). Building a vibration-isolating housing or support system for the mass spectrometer would improve the instrument's performance on ships and in other environments where vibrations are present (Aldrich et al., 1999; Volpe et al., 2001; Dubé et al., 2006; Pratt et al., 2009).

\subsubsection{Parameterization of air-sea exchange and other physical processes}

Accurate parameterization of gas exchange in the presence of ice remains a controversial topic, with some studies showing high rates of gas transfer and others showing low rates (Fanning and Torres, 1991; Papakyriakou and Miller, 2011; Lovely et al., 2015; Butterworth and Miller, 2016). In Chapter 5, I showed that at $>90 \%$ ice cover, the gas transfer velocity for ${ }^{3} \mathrm{He}$ was $\sim 94 \%$ lower than the open water transfer velocity. This result is consistent with a linear scaling of the gas transfer velocity in the presence of ice as a function of the fraction of open water (Takahashi et al., 2009; Butterworth and Miller, 2016). To determine whether this relationship holds at lower fractional ice cover, more tracer release experiments should be conducted.

In many studies (including the ones in this thesis), only one method of measuring gas exchange is used at a time (Else et al., 2011; Rutgers van der Loeff et al., 2014; Lovely et al., 2015). Given the wide range of estimates of gas transfer velocity in the presence of ice, future gas exchange studies using multiple methods simultaneously in ice-covered environments would be highly informative. For example, the dual tracer release method 
could be combined with measurements of radon, stable noble gases at natural abundances, and/or eddy covariance gas fluxes (Rutgers van der Loeff et al., 2014; Butterworth and Miller, 2016; Loose et al., 2016). If the different methods agree, this will improve confidence in the study's results. If the different methods provide contrasting results, the data may may help us to understand biases inherent in different methods and assist in interpreting the results from published studies where only one method is used.

Furthermore, even in open water there is no consensus on the most accurate gas exchange parameterization to use for gases such as $\mathrm{O}_{2}$ that have a large portion of their air-sea flux driven by bubble-mediated exchange (Stanley et al., 2009b; Liang et al., 2013; Emerson and Bushinsky, 2016). In Monterey Bay, I showed that daily measurements of five stable noble gases can be used to evaluate gas exchange parameterizations in coastal waters and that the parameterization of Liang et al. (2013) displayed the most skill in simulating the observations. Using $\mathrm{O}_{2}$ sensors on autonomous Bio-Argo floats, moorings, and gliders, it is possible to quantify net community production throughout the oceans at low cost compared to ship-based time-series (Emerson et al., 2008; Nicholson et al., 2008; Emerson and Bushinsky, 2014; Nicholson et al., 2015; Plant et al., 2016). However, autonomous sensors for dissolved noble gases are not currently available and NCP estimates derived from $\mathrm{O}_{2}$ alone are strongly dependent on the choice of gas exchange parameterization (Plant et al., 2016; Emerson and Bushinsky, 2016). Measurements of natural abundance inert gases are an effective tool for developing and distinguishing between different gas exchange parameterizations, which can subsequently be used in studies where noble gas measurements are not available (Spitzer and Jenkins, 1989; Hamme and Emerson, 2006; Stanley et al., 2006, 2009b).

Because traditional methods of measuring multiple noble gases are very labor- and costintensive, many studies using field-based measurements of multiple noble gases to study physical processes are based on a monthly time-series at one location, or on a single profile (or average profile) at one or multiple locations (Hamme and Emerson, 2002; Hamme and Severinghaus, 2007; Stanley et al., 2009b; Ito et al., 2011; Emerson and Bushinsky, 2016). These studies typically focus on processes operating on weekly to decadal timescales. The gas equilibration mass spectrometer is a valuable tool for studying a wide range of physical processes that affect dissolved gases on short timescales, such as the response of gases to diurnal temperature changes and variable wind speeds.

\subsubsection{Lagrangian studies of gases and productivity}

In Chapters 3-4, I presented a six-day time series from Monterey Bay of $\mathrm{O}_{2}$ concentration and isotopic composition and noble gas concentrations, along with productivity estimates by other methods. I successfully quantified productivity and air-sea exchange using the gas data. However, a longer, fully Lagrangian cruise would likely produce even more valuable 
results.

The duration of the cruise in Monterey Bay was short in relation to the physically dynamic nature of the mixed layer. An upwelling event in the middle of the cruise required us to reset our vertical model for the noble gases, which made it more difficult to see how the various gas exchange models diverged from one another. Furthermore, the fact that we were sampling multiple biogeochemically distinct water masses during the second half of the cruise (because the time-series was not Lagrangian with respect to the mixed layer) made it challenging to quantify productivity by $\mathrm{O}_{2}$ mass balance. In the future, a longer continuous time-series (e.g., multiple weeks) that was Lagrangian with respect to the mixed layer and where the same suite of measurements was collected (noble gases, $\mathrm{O}_{2}$, bottle incubations, and particle export) would be highly informative in understanding the longer-term impacts of physical dynamics on productivity.

An additional challenge in comparing productivity estimates by different methods is that each method integrates over a different spatiotemporal scale (Juranek and Quay, 2005; Hamme et al., 2012; Juranek and Quay, 2013). In Chapter 4, we observed an increase in ${ }^{14} \mathrm{C}$ and ${ }^{15} \mathrm{NO}_{3}{ }^{-}$uptake immediately following the arrival of recently upwelled water, but no increase in productivity by $\mathrm{O}_{2}$ mass balance, nor an increase in particle export. We hypothesized that the lack of a detectable response in $\mathrm{O}_{2}$ may have been due to the longer integration time of the $\mathrm{O}_{2}$ mass balance technique and/or the fact that the $\mathrm{O}_{2}$ measurements, which were performed at all times of the day throughout the cruise track, sampled a wider range of conditions compared to the incubations, which were done with water sampled once per day. A longer cruise that was Lagrangian with respect to the mixed layer would have enabled us to determine whether the increase in $\mathrm{C}$ and $\mathrm{N}$ uptake was coupled to an increase in NCP and particle export flux over longer timescales.

Monthly, multi-year time-series measurements of productivity by multiple methods at the Hawaiian Ocean Time-series site (HOT) and Bermuda Atlantic Time-series Study (BATS) have resulted in improved scientific understanding of the controls on gross, net, and export production in the oligotrophic open ocean (Lohrenz et al., 1992; Karl et al., 1996; Emerson, 2014). Longer duration cruises where productivity is measured at higher frequency would enable scientists to better understand the importance of episodic, high export events in determining the annual or seasonal export flux and the timescales of decoupling between net community production and export production (Burd et al., 2016; Siegel et al., 2016). Such measurements would greatly improve scientists' understanding of the controls on on export production in the ocean and ability to improve satellite- and ecosystem-based models of productivity (Hamme et al., 2012; Emerson, 2014; Burd et al., 2016; Palevsky et al., 2016b; Siegel et al., 2016). 


\subsubsection{Characterization of the isotopic composition of photosynthetic $\mathrm{O}_{2}$}

One promising area of future research is paired measurements of the triple oxygen isotopic composition of $\mathrm{H}_{2} \mathrm{O}$ and $\mathrm{O}_{2}$. In Chapter 5 , one source of error in our work was uncertainty in the triple oxygen isotopic composition of $\mathrm{H}_{2} \mathrm{O}$, which is the substrate for photosynthetic $\mathrm{O}_{2}$. At the time we conceived and conducted this study, very few labs worldwide analyzed $\mathrm{H}_{2} \mathrm{O}$ using methods with sufficient precision to resolve the ${ }^{17} \mathrm{O}$-excess in $\mathrm{H}_{2} \mathrm{O}$. Published methods involved the generation of hazardous chemicals (hydrofluoric acid) and required the conversion of $\mathrm{H}_{2} \mathrm{O}$ to $\mathrm{O}_{2}$ prior to analysis, precluding the simultaneous measurement of $\delta^{17} \mathrm{O}$ and $\delta^{2} \mathrm{H}$ (Luz and Barkan, 2005). The recent development of commercially-available laser-based sensors that simultaneously measure the ${ }^{17} \mathrm{O}$-excess and $\delta^{2} \mathrm{H}$ of $\mathrm{H}_{2} \mathrm{O}$ directly, without complicated sample processing (Berman et al., 2013; Steig et al., 2014), will greatly increase the number of laboratories making this measurement and reduce analytical costs.

Laboratory experiments (e.g. with microbial cultures) could be performed to verify how the isotopic composition of photosynthetic $\mathrm{O}_{2}$ changes as a function of the isotopic composition of $\mathrm{H}_{2} \mathrm{O}$ (Eisenstadt et al., 2010; Luz and Barkan, 2011). These experiments would help to verify the approach used in Chapter 5 and also serve as an additional method of evaluating the equations used for quantifying GPP from the triple oxygen isotopic composition of $\mathrm{O}_{2}$; if the ${ }^{17} \Delta$ of photosynthetic $\mathrm{O}_{2}$ does not vary with the isotopic composition of $\mathrm{H}_{2} \mathrm{O}$ in the expected manner, this result may indicate some inaccuracies in the equations and/or constants used to calculate GPP. Furthermore, although the ratio of respiratory fractionation factors for ${ }^{18} \mathrm{O}-\mathrm{O}_{2}$ and ${ }^{17} \mathrm{O}-\mathrm{O}_{2}$ appears to be nearly constant among a variety of organisms (including autotrophs and heterotrophs) (Luz and Barkan, 2005), the photosynthetic isotopic fractionation varies between classes of phytoplankton and also with the ambient $\mathrm{O}_{2}$ concentration (Helman et al., 2005; Eisenstadt et al., 2010; Barkan and Luz, 2011). More experimental work is needed to better characterize the isotopic composition of photosynthetic $\mathrm{O}_{2}$ in a variety of systems.

I recommend that in the future, when the triple oxygen isotope tracer of photosynthesis is used in locations where the isotopic composition is expected to differ significantly from VSMOW, the ${ }^{17} \mathrm{O}$-excess of water should ideally be measured. Furthermore, I concur with other authors that all data needed to calculate productivity (e.g., $\delta^{17} \mathrm{O}-\mathrm{O}_{2}, \delta^{18} \mathrm{O}-\mathrm{O}_{2},{ }^{17} \Delta$, $\left[\mathrm{O}_{2}\right]$, temperature, salinity, and depth, as well as the wind speed record used and/or gas transfer velocity) should be archived so that productivity can be recalculated in the future based on new consensus and information about the most accurate equations and constants to use in these calculations (Hamme et al., 2012; Juranek and Quay, 2013). To this end, I have made the field data from Monterey Bay publicly available (Manning et al., 2016b,c) and also provided the MATLAB code that I used to calculate air-sea gas fluxes and gross primary production (Manning and Nicholson, 2016; Manning and Howard, 2016). The data associated with Chapter 5 will be provided when it is submitted to a journal for peer review. 
Public databases of ${ }^{17} \mathrm{O}$-excess and $\delta^{17} \mathrm{O}-\mathrm{H}_{2} \mathrm{O}$ and spatiotemporal isotope models (isoscapes), similar to the references used to estimate $\delta^{18} \mathrm{O}-\mathrm{H}_{2} \mathrm{O}$ in Chapter 5 , would also be highly beneficial to the community of scientists working with triple oxygen isotopes (Schmidt et al., 1999; LeGrande and Schmidt, 2006; Bowen, 2010; Bowen and Good, 2015; IAEA/WMO, 2016).

\subsection{Broader implications}

Improved understanding of gas exchange mechanisms and biological productivity in coastal zones is needed to reduce uncertainty in carbon cycling near air-sea and land-sea interfaces. These interfaces are dynamic regions where carbon fluxes are large and poorly quantified. Although the field measurements in this thesis focussed on small regions, some of the results of this thesis have improved our broader understanding of the chemical, biological, and physical processes affecting the carbon cycle.

In Chapter 3, I presented a six-day time-series of noble gases in an upwelling-influenced coastal zone and showed that the gas exchange parameterization of Liang et al. (2013) displayed the most skill in simulating the $\mathrm{He}$ and $\mathrm{Ne}$ observations. These results indicate that gas exchange parameterizations not explicitly developed for the coastal ocean may be applicable in such environments. Recent studies have indicated that the parameterization of Liang et al. (2013) is also appropriate for simulating $\mathrm{O}_{2}$ in the northeast Pacific Ocean (Emerson and Bushinsky, 2016; Plant et al., 2016). All of these studies demonstrate that a parameterization with no bubble flux and too much bubble flux will both result in errors in the calculated air-water flux for low solubility gases. These and other studies provide insight into the most appropriate gas exchange parameterization to use in global biogeochemical models, and when interpreting data sets where inert gas measurements are not available, such as measurements of $\mathrm{O}_{2}$ from moorings and BioArgo floats (Hamme and Emerson, 2006; Emerson and Stump, 2010; Plant et al., 2016).

In Chapter 4, I demonstrated, for the first time in a coastal nitrogen-limited system, that $\mathrm{O}_{2} / \mathrm{Ar}$ and ${ }^{15} \mathrm{NO}_{3}^{-}$uptake-based methods of estimating net community production can give equivalent results in a steady state system. Furthermore, the ${ }^{15} \mathrm{NO}_{3}{ }^{-}$tracer loadings represented 51(8) \% of the ambient nutrient concentrations, which is substantially higher than the typical recommendation to limit tracer loadings to $<10 \%$ of the ambient concentration (Dugdale and Goering, 1967). This result indicates that ${ }^{15} \mathrm{NO}_{3}{ }^{-}$uptake incubations are an effective method of estimating productivity in low nitrogen environments. Comparisons of ${ }^{15} \mathrm{NO}_{3}{ }^{-}$uptake and $\mathrm{O}_{2} /$ Ar-based net community production in a wide range of environments would advance our understanding of the benefits and limitations of both methods, and provide more accurate recommendations on the optimal tracer loading. Additionally, I demonstrated that we obtain a fuller understanding of the carbon cycle by using multi- 
ple methods of estimating productivity simultaneously. For example, incubations provide real-time estimates of productivity once per day, whereas the $\mathrm{O}_{2}$ mass balance technique provides information on submesoscale variability in productivity but requires corrections for physically-driven $\mathrm{O}_{2}$ fluxes, which can be challenging to estimate.

In Chapter 5, I showed that at $>90 \%$ ice cover, the effective gas transfer velocity can be calculated by multiplying the open water gas transfer velocity by the fraction of open water. This result is significant because the few existing field-based measurements of gas transfer velocity in the presence of ice gave a wide range of values. Recent estimates of the gas transfer velocity for $\mathrm{CO}_{2}$ from eddy covariance measurements at 0 to $>90 \%$ ice cover in the Southern Ocean also support a linear scaling of gas transfer velocity based on ice cover (Butterworth and Miller, 2016). Some earlier eddy covariance studies using open path sensors that showed high $\mathrm{CO}_{2}$ fluxes in partially ice-covered waters may be have overestimated the flux due to water vapor interference on the $\mathrm{CO}_{2}$ signal (Blomquist et al., 2014; Butterworth and Miller, 2016). Accurate parameterization of gas exchange in partially ice-covered waters is needed because ice cover in polar regions is rapidly changing. In order to understand whether the Arctic Ocean carbon sink is decreasing or increasing, we must understand how changes in ice cover affect the gas transfer velocity. The results in this thesis and those of Butterworth and Miller (2016) indicate that the rate of gas transfer through ice is negligible relative to gas transfer through water and that gas transfer is not enhanced in the presence of ice, which suggests that an ice-free Arctic Ocean will have substantially higher gas transfer velocities than an ice-covered Arctic Ocean. However, more field-based measurements of the gas transfer velocity at intermediate ice cover by a variety of methods would help to validate these results.

Another important implication of this work is the importance of determining whether a system is at steady state when quantifying productivity from gas tracers. In many studies, time-series measurements are not available and it is necessary to assume the system is at steady state in order to estimate GPP and NCP from gas tracer measurements. In Chapter 5 , we measured the rate of change in the gas tracers with time and found that the non-steady state terms were a significant contributor to the overall GPP and NCP. In Chapter 4, we found that the system was in steady state at the start of the time-series, and at the end of the time-series our measurements indicated that the system was not at steady state, but we were unable to quantify the non-steady state term, in part because we sampled a variety of different water masses with different biogeochemical properties, rather than only sampling one water mass. Although we were not able to quantify the non-steady state term at the end of the time-series, having the time-series helped us to interpret the data more accurately. Specifically, the time-series measurements indicated that the steady-state GPP and NCP were lower than the true GPP and NCP.

In summary, the results in this thesis provide insight into which gas exchange parame- 
terizations are most accurate to use in coastal and ice-covered waters, which can be applied in future studies. They demonstrate that ${ }^{15} \mathrm{~N}$ incubation- and gas tracer-based estimates of export production can give equivalent results in a steady state nitrogen-limited system, which validates both approaches. Additionally, they show that time-series measurements improve the accuracy of GPP and NCP estimates in dynamic coastal systems, which will help inform the design of future field campaigns. 
Appendix A

Supplemental Information for Chapter 2:

Continuous measurements of dissolved $\mathrm{Ne}, \mathrm{Ar}, \mathrm{Kr}$, and $\mathrm{Xe}$ ratios with a field-deployable gas equilibration mass spectrometer 


\section{A.1 Introduction}

In the this appendix, we present ancillary information that will be useful to researchers who plan to build a gas equilibration mass spectrometer (GEMS) and/or modify the method described in the main paper. We describe alternate configurations that were tested, and the instrument settings used for the mass spectrometer. We provide photos of the GEMS (Figures A-5-A-7), a schematic of the custom-made getter chambers (Figure A-8), and tables of the parts used (Tables A-1 and A-2). We show the field data collected in Waquoit Bay before and after calibration with discrete samples, along with the calibration curves used. Additionally, we discuss the effect of vibrations on the data quality.

\section{A.2 Alternative configurations tested}

Water flow configuration: On a research vessel, we tested a setup where the underway seawater stream flowed directly from the filter canisters into the membrane contactor (avoiding the need for overflowing water, thereby simplifying the setup). However, we found that the gas mole ratios measured were inaccurate in this configuration, based on comparison of the GEMS data with discrete samples. We hypothesize that back pressure within the underway line may have altered the equilibration kinetics within the membrane contactor. By pumping the water into a bucket, which is open to the ambient air, and then sampling from the bucket, we allow the water to depressurize before it enters the membrane contactor. We minimize gas exchange within the bucket by using a high water flow rate, so that the residence time of water in the bucket is $<30 \mathrm{~s}$, and we pump water from the bottom of the bucket into the membrane contactor.

Cartridge selection: We tested smaller membrane contactor cartridges from LiquiCel (MiniModules and MicroModules) but found that they did not achieve full equilibrium, even with a much longer capillary. The LiquiCel ExtraFlow configuration includes a center baffle that diverts the water flow radially (perpendicular to the lumens), which improves gas transfer efficiency compared to a design in which the water flow is parallel to the lumen orientation (e.g., LiquiCel MiniModule). Additionally, the ExtraFlow cartridge has a low headspace to water volume ratio $\left(150 \mathrm{~cm}^{3}\right.$ headspace, $400 \mathrm{~cm}^{3}$ water $)$ compared to the MiniModule, which improves the e-folding response time. We also tested a nonporous silicone membrane contactor (PermSelect PDMSXA-8300) but found that it did not fully equilibrate

and its performance was substantially worse compared to a similarly-sized porous membrane contactor.

Capillary dimensions and material: We tested a wider diameter and/or shorter cap- 
illary, which would increase the flow rate and therefore the the signal measured by the mass spectrometer, but were unable to achieve full equilibrium at a higher gas flow rate, based on tests with equilibrated water. We found the data was most consistent with time, and had less of an offset between air and headspace, when we used deactivated fused silica capillary as compared to untreated fused silica. The untreated silica adsorbs water vapor from the gas stream, whereas the deactivated tubing (in this case, phenyl methyl deactivation) has an interior coating surface that makes the capillary inert to water and other chemicals containing - $\mathrm{OH}$ groups.

Vacuum pumping speed: Since we could not increase the flow rate into the mass spectrometer, we opted to reduce the speed at which gas was pumped away after entering the mass spectrometer by including an orifice in the design of the manifold, between the mass spectrometer and the vacuum pumps. We created the orifice by replacing a standard 4.5" CF flange gasket with a blank off gasket drilled with a 1" diameter hole. This orifice reduces the pumping speed by a factor of 25. Vacuum is provided by an Agilent TPS-Compact with IDP-3 dry scroll pump and Turbo-V 301 Navigator turbomolecular pump; we selected this system due to its small size (46 cm L x $33 \mathrm{~cm} \mathrm{~W} \mathrm{x} \mathrm{12"} \mathrm{H,} 18 \mathrm{~kg}$ ). We also tested the Turbo-V 81 MacroTorr turbomolecular pump, which has a slower pumping speed compared to the 301 Navigator model, but found this vacuum pumping system to be more prone to failure and therefore did not use it in our final configuration.

Getters: Other investigators have used SAES Getters alloys St101 and St707 to purify gas for the measurement of noble gases in water and ice samples (Visser et al., 2013; Stanley et al., 2009a; Hamme and Severinghaus, 2007; Severinghaus et al., 2003; Beyerle et al., 2000). We did not test alloy St101 because its activation temperature is $750{ }^{\circ} \mathrm{C}$, which makes the system less safe to operate outside of the lab. Instead, we tested alloys St707 and St2002, which have activation temperatures of 450 and $400{ }^{\circ} \mathrm{C}$ respectively. We selected alloy St2002, which contains $\mathrm{Zr}, \mathrm{V}, \mathrm{Mg}, \mathrm{Fe}$, and rare earth elements, due to its improved removal efficiency for $\mathrm{N}_{2}$ (Figure A-1). Nitrogen is the most difficult gas to remove, due to its strong triple bond. The long, cylindrical design of the getter cans (Figure A-8) increases the exposure of the gas stream to the getter surface, thereby improving the removal efficiency.

We use SAES Getters alloy St2002 pieces (cylindrical pellets, $6 \mathrm{~mm}$ diameter x $2 \mathrm{~mm}$ long), with approximately $100 \mathrm{~g}$ of getter in the first, heated chamber, and $30 \mathrm{~g}$ of getter in the second, room temperature chamber. The long, cylindrical design of the getter chambers (1" diameter x 4" long and 3/4" diameter x 3" long) increases the contact between the gas stream and the getters, thereby improving the removal efficiency. After placing the getter pieces into the chambers, the initial activation of the getters is done on a vacuum pumping system without the mass spectrometer installed, due to concerns that the mass 

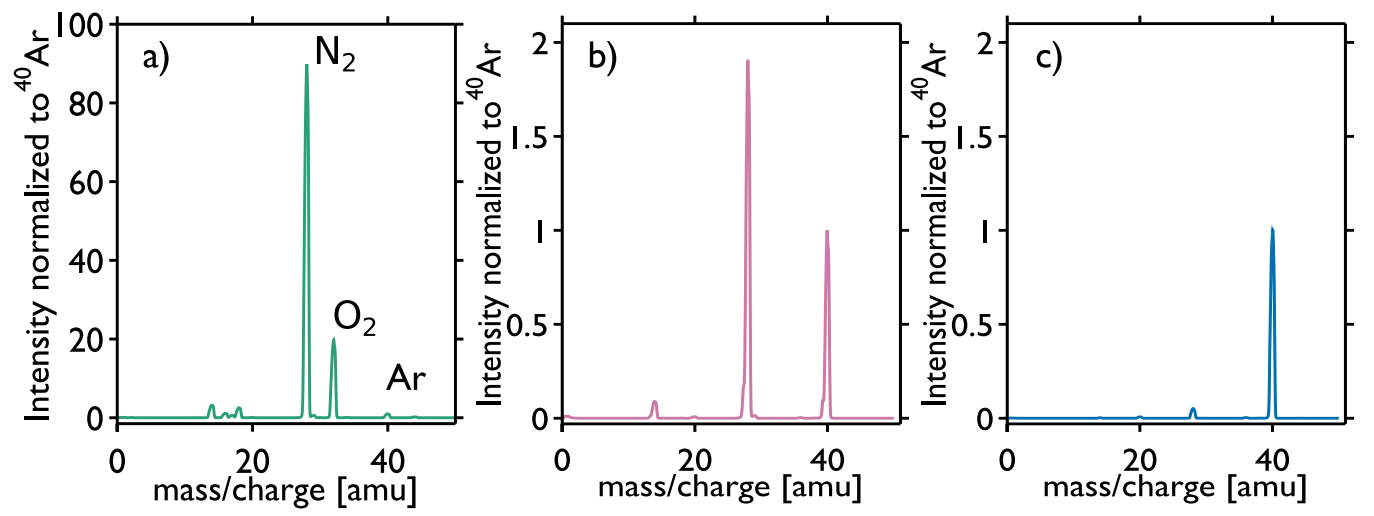

Figure A-1: Mass spectra of air with a) no purification, b) St707 getter, and c) St2002 getter, normalized to ${ }^{40} \mathrm{Ar}$. Note the changes in the $\mathrm{N}_{2} / \mathrm{O}_{2} / \mathrm{Ar}$ ratio.

spectrometer could be contaminated by the large quantities of gas released, and any loose getter particles in the cans. Subsequent re-activations release much smaller quantities of gas and can be performed with the getters attached to the mass spectrometer, as long as the mass spectrometer is turned off. The getters are reactivated any time that the getters have been exposed to air at atmospheric pressure, or when the peak height for $\mathrm{N}_{2}(\mathrm{~m} / \mathrm{z}=28)$ becomes greater than the peak height for $\operatorname{Ar}(\mathrm{m} / \mathrm{z}=40)$, which occurs approximately once per month when the system is in continuous use. Replacement of the getters is performed roughly once per year, when reactivation fails to reduce the $\mathrm{N}_{2}$ peak height to a satisfactory level, because the getters are saturated with gas.

Mass spectrometer: We selected the Hiden Analytical HAL 3F RC 201 quadrupole mass spectrometer due to its unique capability to vary the electron energy during an experiment, without a loss of instrument stability. The manufacturer's software (MASsoft Professional 7) allows a different electron energy to be used for each mass measured in a multiple ion detection experiment. Preliminary method development was done using a Pfeiffer PrismaPlus 200 QMS, which requires the same electron energy to be used for all masses within an experiment. In this case, we measured all masses with an electron energy of $35 \mathrm{~V}$, which enabled accurate measurement of ${ }^{22} \mathrm{Ne}$, but degraded the precision for ratios with Xe to be worse than $2 \%$, in comparison to $0.7 \%$ or better using the Hiden QMS. The disadvantage of the Hiden QMS is that it is more sensitive to vibrations than the Pfeiffer.

Measurement of other gases: We have used the GEMS, with the getter chambers removed, to obtain high-accuracy, high-precision measurements of the dissolved $\mathrm{O}_{2} / \mathrm{Ar}$ ratio. We have also attempted to measure $\mathrm{N}_{2} / \mathrm{Ar}$, but found there was a systematic offset between the GEMS and discrete samples run on an isotope ratio mass spectrometer. We do not believe this offset is caused by incomplete equilibration, since the GEMS is able to 
equilibrate $\mathrm{Ne}$, which is $30 \%$ less soluble than $\mathrm{N}_{2}$. We hypothesize that matrix differences between the headspace and air (e.g., pressure and/or humidity differences) may cause this offset. These matrix differences would be reduced or eliminated for the noble gas GEMS since the getters purify the air and headspace gas, removing nearly all of the non-noble gas content before the gas stream enters the ion source. Further testing of the equilibration components and the GEMS for measurement of other gases should be conducted.

\section{A.3 Details on water pumps, dessicant, and membrane con- tactor}

\section{A.3.1 Choice of water pumps}

To pump the water from the bucket through the membrane contactor, we use a suction shoe gear pump from Micropump. The pump is the same type used by Cassar et al. (2009), however, we selected a head that would produce a higher flow rate $\left(1.17 \mathrm{~cm}^{3} /\right.$ revolution, or up to $3500 \mathrm{~cm}^{3} \mathrm{~min}^{-1}$ ). Pumping the water through the gear pump (which is typically at a different height than the bucket water level, though within $\sim 1 \mathrm{~m}$ ) could potentially alter the dissolved gas content. Therefore, when we collected discrete samples to determine the accuracy of the method, we collected these samples from a separate sampling port, using water that did not pass through the gear pump. Since, as shown in the main paper, the two methods agree well, we do not think that the gear pump affects the gas mole ratios.

For the field experiment, we used a Goulds submersible well pump (SB Bruiser 5-18 GPM), capable of flow rates from $20000-70000 \mathrm{~cm}^{3} \mathrm{~min}^{-1}$, along with a pressure regulating valve. This submersible pump is ideal for dissolved gas measurements when the water needs to be pumped long distances and when the height of the water changes, since it pushes, rather than pulls, the water along its flow path. Pushing the water helps to ensure the gas content is not altered and prevents cavitation.

\section{A.3.2 Maintaining a dry environment in the headspace}

The headspace gas is continuously recirculated and dried to reduce the condensation of water vapor on the headspace side. We use two desiccants: a Nafion tube surrounded by molecular sieves, followed by a Drierite tube. Using the two desiccants in sequence improves the water removal efficiency and reduces the replacement frequency. Both desiccants have color-changing indicators to alert when the desiccant is saturated and should be reactivated, which is typically every $2-7$ days. The frequency of replacement depends greatly on the temperature of the water flowing through the cartridge (condensation forms more rapidly for warmer water). Although the desiccants reduce the formation of condensation, there is 
still some accumulation of water on the headspace side. During operation, we orient the membrane contactor at a $45^{\circ}$ angle (Figure A-5), so that liquid water does not enter the recirculation loop.

When the desiccants are replaced, the membrane contactor is dried to remove condensation by flowing a dry gas, such as $\mathrm{N}_{2}$, through the contactor. Gas enters one of the headspace ports, and the second headspace port is plugged. This setup forces the dry gas to pass through the pores in the membrane and out the water ports, thereby flushing out any liquid water that has clogged the pores. The membrane contactor is oriented so that water can drain out the water ports. After drying the cartridge for $\sim 30 \mathrm{~min}$, the contactor is inverted and gas is flushed through the other headspace port. To reduce downtime, we have a second membrane contactor and desiccant set available, so that the new set can be installed as soon as the old set is removed. When the new cartridge is installed, we allow water to flow through the membrane contactor for at least $1 \mathrm{~h}$ before sampling from the headspace of the membrane contactor, to ensure all bubbles have been flushed out.

The Drierite and molecular sieves can be reactivated by heating in an oven at 210 and $250{ }^{\circ} \mathrm{C}$, respectively, until the indicator has turned from pink to blue. Both dessicants should be allowed to cool in closed, gastight containers.

\section{A.3.3 Potential permeation of $\mathrm{He}$ and $\mathrm{Ne}$}

One concern with measurement of the lighter noble gases is the potential for permeation of the gas through the membrane housing and the capillary. We confirmed that the mass spectrometer housing and the getter chambers were leak tight, by spraying He along these components while the system was isolated from air (capillary valve was closed) and observing that the peak height at $\mathrm{m} / \mathrm{z}=4$ did not increase. However, He gas was detected by the mass spectrometer within minutes when sprayed near the capillary and the membrane contactor. Other investigators have reported on permeation of He through fused silica capillaries (Cahill and Tracy, 1998; Swets et al., 1961). We concluded that accurate measurement of He with the GEMS would require changing the materials used for the capillary and/or the membrane contactor (e.g., to stainless steel). Machler et al. (2012) used a Liqui-Cel membrane contactor for equilibration of $\mathrm{He}$, with accuracy of $3 \%$, but the capillary had a greater wall thickness (0.73 $\mathrm{mm}$ versus $0.16 \mathrm{~mm}$ ) and was made of PEEK rather than fused silica.

We sprayed pure Ne gas at various points along the sample pathway (the tubing, the capillary, the sample cartridge, the getters, etc.) for $15 \mathrm{~min}$. We continuously monitored the signal for Ne on the mass spectrometer. No change was observed in the Ne peak height. We conclude that the sampling system can be considered leak tight with respect to Ne, for the residence time of $\mathrm{Ne}$ in the sampling system. 


\section{A.4 Mass spectrometer settings}

The typical ion source settings and the acquisition settings for an experiment measuring the noble gases are summarized in Tables A-1 and A-2. Each noble gas is measured with a different amplifier (range) based on its abundance, and we keep the range fixed for each mass during the experiment by deselecting the auto range setting. We deselect the auto zero setting to speed up the acquisition; the auto zero would perform a zeroing function before measuring each gas during each measurement cycle to correct for leakage currents and amplifier offsets, and this zeroing function lasts at least as long as the measurement. Disabling the auto zero function does not affect the accuracy of the method, since we are measuring the noble gas mole ratios rather than their absolute abundances. For Xe, we measure both ${ }^{129} \mathrm{Xe}$ and ${ }^{132} \mathrm{Xe}$ for $10 \mathrm{~s}$ each and take the average of the two peak heights. $\mathrm{Xe}$ is the least abundant of the gases we measure and the software limits the acquisition to $16 \mathrm{~s}$ per mass; therefore, by measuring ${ }^{129} \mathrm{Xe}$ and ${ }^{132} \mathrm{Xe}$ for $10 \mathrm{~s}$ each we improve the precision of the method.

The mass spectrometer settings for an experiment to measure noble gas mole ratios are shown in Table A-2. For $\mathrm{m} / \mathrm{z}=22$ we use an electron energy of $35 \mathrm{~V}$ and emission current of $500 \mu \mathrm{A}$. The rest of the ions are measured with an electron energy of $55 \mathrm{~V}$ and emission current of $1000 \mu \mathrm{A}$. For $\mathrm{m} / \mathrm{z}=22$, the lower electron energy is used to avoid formation of doubly charged $\mathrm{CO}_{2}$, which has the same $\mathrm{m} / \mathrm{z}$ as ${ }^{22} \mathrm{Ne}$ (Hamme and Emerson, 2004b), and the lower emission current is necessary when operating at reduced electron energy to avoid overloading the filament. The settle time describes how long the instrument waits before starting the next measurement, and we use a longer settle time for ${ }^{22} \mathrm{Ne}$ and Ar to allow the instrument to stabilize after a change in electron energy. Each scan (measurement of all six masses) takes approximately $1 \mathrm{~min}$. Note that in some experiments in the main text (notably the lab accuracy test) we measured ${ }^{36} \mathrm{Ar}$ at a range of $10^{-8}$ (typical intensity $2 \mathrm{x}$ $10^{-9}$ Torr) rather than measuring ${ }^{38} \mathrm{Ar}$ at a range of $10^{-10}$. We found ${ }^{38} \mathrm{Ar}$ was preferable as it could be measured on the same range as ${ }^{84} \mathrm{Kr}$. Minimizing the number of amplifiers used during the scan helps to minimize nonlinearities in the detector response.

Table A-1: Ion source settings

\begin{tabular}{ll}
\hline Variable & Setting \\
\hline focus & $-65 \mathrm{~V}$ \\
cage & $3 \mathrm{~V}$ \\
electron energy & 35 or $55 \mathrm{~V}$ \\
emission current & 500 or $1000 \mu \mathrm{A}$ \\
multiplier & $1100 \mathrm{~V}$ \\
\hline
\end{tabular}


Table A-2: Acquisition settings for MID (multiple ion detection) experiment

\begin{tabular}{llllllll}
\hline Gas & $\begin{array}{l}\text { Mass } \\
(\mathrm{m} / \mathrm{z})\end{array}$ & $\begin{array}{l}\text { Dwell time } \\
(\mathrm{ms})\end{array}$ & $\begin{array}{l}\text { Settle time } \\
(\mathrm{ms})\end{array}$ & $\begin{array}{l}\text { Range } \\
(\mathrm{Torr})\end{array}$ & $\begin{array}{l}\text { Electron } \\
\text { energy }(\mathrm{V})\end{array}$ & $\begin{array}{l}\text { Emission } \\
\text { current }(\mu \mathrm{A})\end{array}$ & $\begin{array}{l}\text { Typical } \\
\text { intensity (Torr) }\end{array}$ \\
\hline $\mathrm{Ne}$ & 22 & 10000 & 4000 & $10^{-11}$ & 35 & 500 & $8 \times 10^{-12}$ \\
$\mathrm{Ar}$ & 38 & 2000 & 4000 & $10^{-10}$ & 55 & 1000 & $4 \times 10^{-10}$ \\
$\mathrm{Kr}$ & 84 & 5000 & 1000 & $10^{-10}$ & 55 & 1000 & $1 \times 10^{-10}$ \\
${ }^{129} \mathrm{Xe}$ & 129 & 10000 & 1000 & $10^{-11}$ & 55 & 1000 & $3 \times 10^{-12}$ \\
${ }^{132} \mathrm{Xe}$ & 132 & 10000 & 1000 & $10^{-11}$ & 55 & 1000 & $3 \times 10^{-12}$ \\
\hline
\end{tabular}

\section{A.4.1 Elimination of doubly-charged $\mathrm{CO}_{2}$}

In order for doubly-charged $\mathrm{CO}_{2}$ to be formed in the QMS, the energy of the electron beam must be at least $37 \mathrm{~V}$, which is the minimum energy required to eliminate two electrons from $\mathrm{CO}_{2}$. We measure ${ }^{22} \mathrm{Ne}$ at an electron energy of $35 \mathrm{~V}$ to prevent doubly-charged $\mathrm{CO}_{2}$ from forming. To verify that doubly-charged $\mathrm{CO}_{2}$ is not formed at the reduced electron energy, we sampled from a gas cylinder of 360 ppmv $\mathrm{CO}_{2}$ in $99.9990 \%$ purity He using the same capillary used for sampling air. We found that when the ionization energy was $35 \mathrm{~V}$, the signal at $\mathrm{m} / \mathrm{z}=22$ was not distinguishable from the baseline at $\mathrm{m} / \mathrm{z}=23.5$, indicating that doubly-charged $\mathrm{CO}_{2}$ was not detected. When the ionization energy was $70 \mathrm{~V}, \mathrm{~m} / \mathrm{z}=$ 22 had a substantial peak that was 11 times the background and $75 \%$ the height of the peak for singly-charged $\mathrm{CO}_{2}$ at $\mathrm{m} / \mathrm{z}=44$. Thus we conclude that ${ }^{22} \mathrm{Ne}$ can be accurately measured with the Hiden QMS at an ionization energy of $35 \mathrm{~V}$.

We do not measure ${ }^{20} \mathrm{Ne}$ (which is 10 times more abundant than ${ }^{22} \mathrm{Ne}$ ) due to the isobaric interference from singly-charged $\mathrm{H}_{2} \mathrm{O}$ at $\mathrm{m} / \mathrm{z}=20\left({ }^{18} \mathrm{O}-\mathrm{H}_{2} \mathrm{O}, 0.2 \%\right.$ abundance $)$. If we were able to completely remove $\mathrm{H}_{2} \mathrm{O}$, we could measure ${ }^{20} \mathrm{Ne}$ by reducing the electron energy below $43 \mathrm{~V}$, the minimum required to form doubly-charged ${ }^{40} \mathrm{Ar}$. In the current configuration, at an electron energy of $40 \mathrm{~V}, \mathrm{H}_{2} \mathrm{O}$ will contribute $\sim 10 \%$ to the peak height at $\mathrm{m} / \mathrm{z}=20$ (based on the peak height at $\mathrm{m} / \mathrm{z}=18$ ), and the peak height will vary with time, making it impossible to achieve measurements of ${ }^{20} \mathrm{Ne}$ with better than $1 \%$ accuracy and precision.

\section{A.5 Field data}

\section{A.5.1 Data from Waquoit Bay, MA}

In the main manuscript (Figure 2-5), we show data from a field experiment where the instrument was installed on land and water was pumped to shore. We achieved similar accuracy in the lab and field for $\mathrm{Ne} / \mathrm{Kr}, \mathrm{Ne} / \mathrm{Ar}$, and $\mathrm{Ar} / \mathrm{Kr}$ when air was used as the only calibration standard (field accuracy of $0.7 \%$ or better, compared to lab accuracy of $0.9 \%$ or better). However, we achieved worse accuracy for the ratios with $\mathrm{Xe}(\mathrm{Ne} / \mathrm{Xe}, \mathrm{Ar} / \mathrm{Xe}$, 
and $\mathrm{Kr} / \mathrm{Xe}$ ) in the field when air was used as the only calibration standard (field accuracy of $2.0-2.5 \%$, compared to lab accuracy of $0.8 \%$ or better). However, after calibration with discrete samples, the accuracy for the ratios with Xe improved to $0.6 \%$ or better. We did not calibrate the measured $\mathrm{Ne} / \mathrm{Kr}, \mathrm{Ne} / \mathrm{Ar}$, and $\mathrm{Ar} / \mathrm{Kr}$ ratios with the discrete sample data since the accuracy with air calibration only was very good. Here we show the calibration curve that was used to calibrate the GEMS data (Figure A-2). We also show the data before calibration (Figure A-3) and after calibration (Figure A-4). In these figures, the uncertainties in the gas ratios are estimated at 2.0, 1.0, 0.4, 2.0, 1.0, and $2.0 \%$ for $\mathrm{Ne} / \mathrm{Xe}, \mathrm{Ne} / \mathrm{Kr}, \mathrm{Ne} / \mathrm{Ar}$, $\mathrm{Ar} / \mathrm{Xe}, \mathrm{Ar} / \mathrm{Kr}$, and $\mathrm{Kr} / \mathrm{Xe}$, respectively. This is because the published seawater solubilities of $\mathrm{Kr}$ and Xe have large uncertainties compared to the solubilities of $\mathrm{Ne}$ and Ar, which were redetermined more recently (Hamme and Emerson, 2004a; Hamme and Severinghaus, 2007; Stanley et al., 2009b).

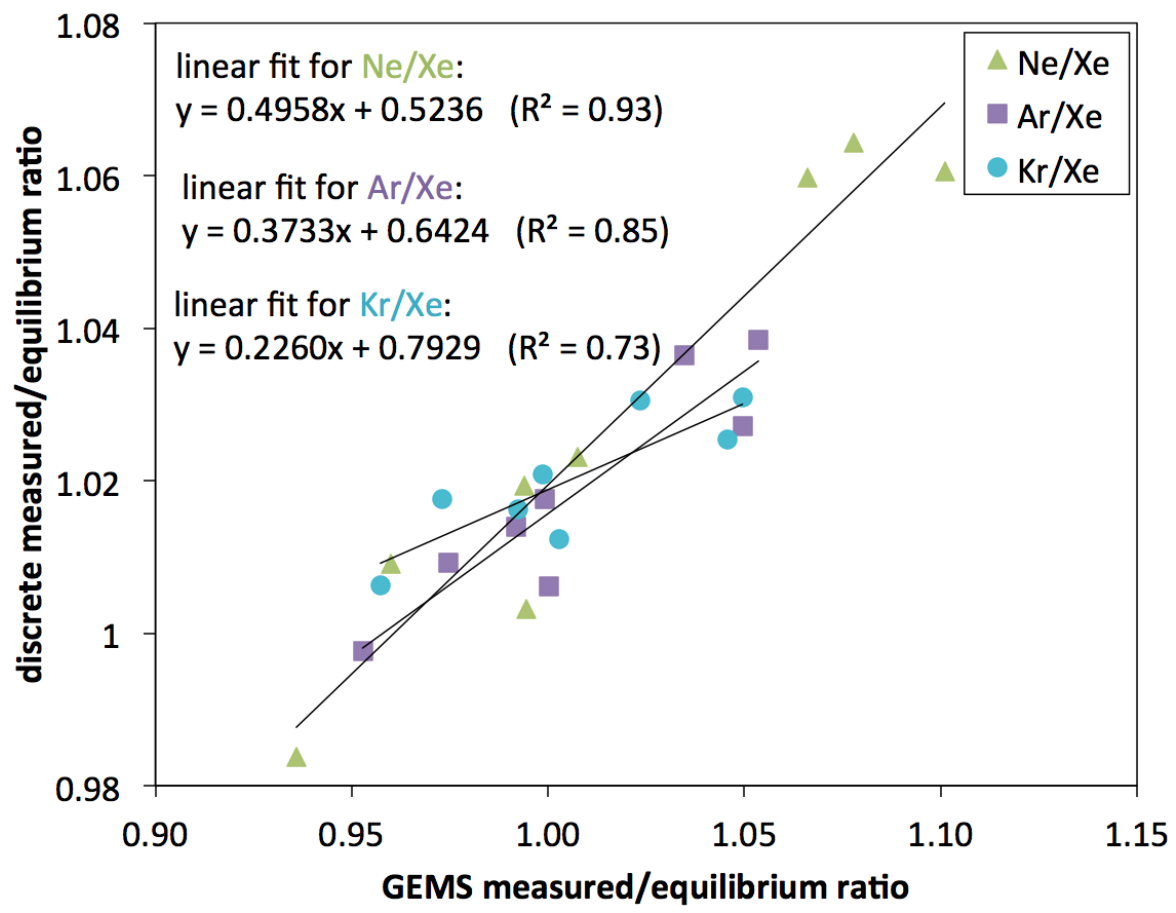

Figure A-2: We constructed a calibration curve for $\mathrm{Ne} / \mathrm{Xe}, \mathrm{Ar} / \mathrm{Xe}$, and $\mathrm{Kr} / \mathrm{Xe}$ by plotting the measured ratio, normalized to equilibrium, that was measured using the discrete samples versus the GEMS at the time of sample collection.

\section{A.5.2 Data on a moving vessel and the impact of vibration-induced noise}

We have installed the instrument on two research vessels, the R/V Tioga ( $\sim 60 \mathrm{ft}$, single hull) and the $\mathrm{R} / \mathrm{V}$ Western Flyer ( $\sim 17 \mathrm{ft}$, twin hull). On both vessels, we observed an increase in noise when the ship's motor was on and the ship was moving, compared to when the ship was docked. On the R/V Tioga, which can travel at speeds up to $18 \mathrm{kt}$, we observed spikes 

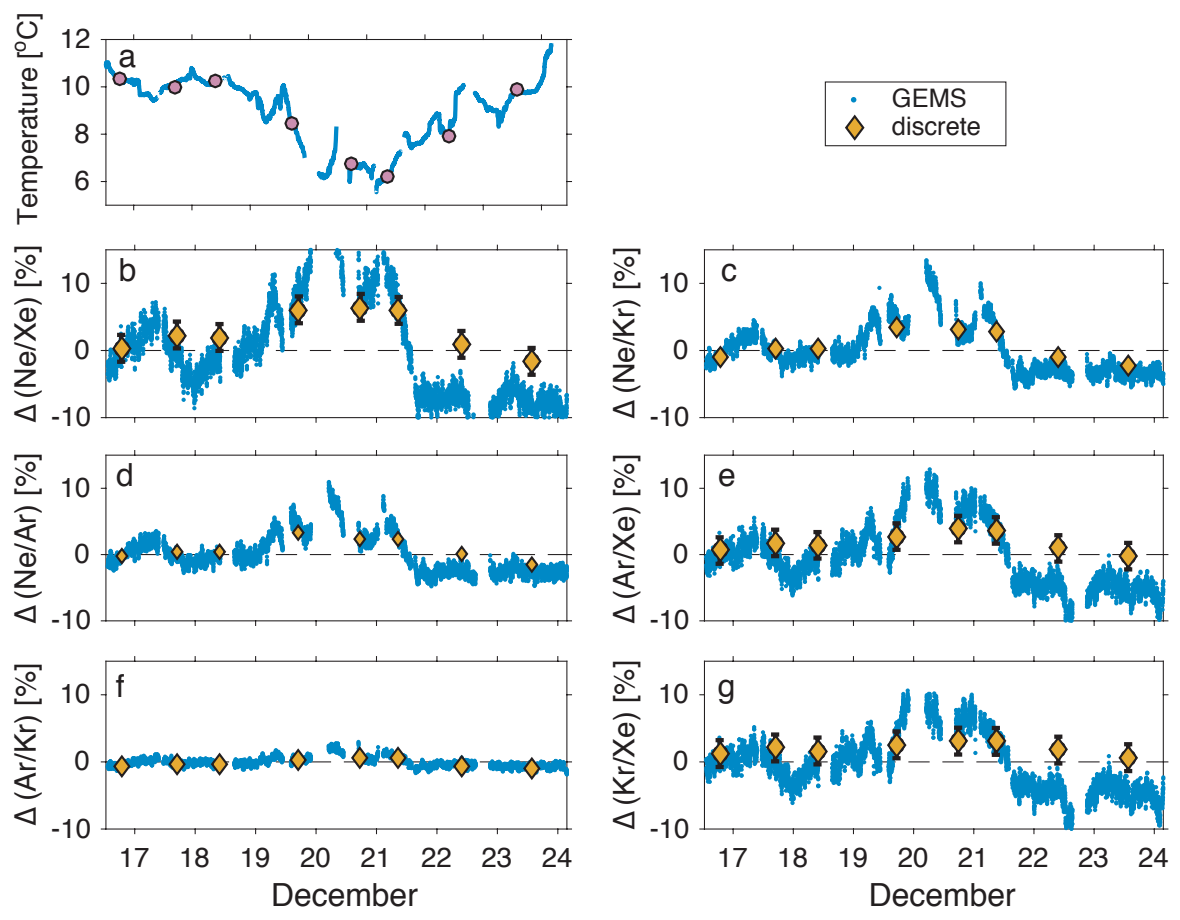

Figure A-3: Field data from GEMS and discrete samples, calibrated only with air.
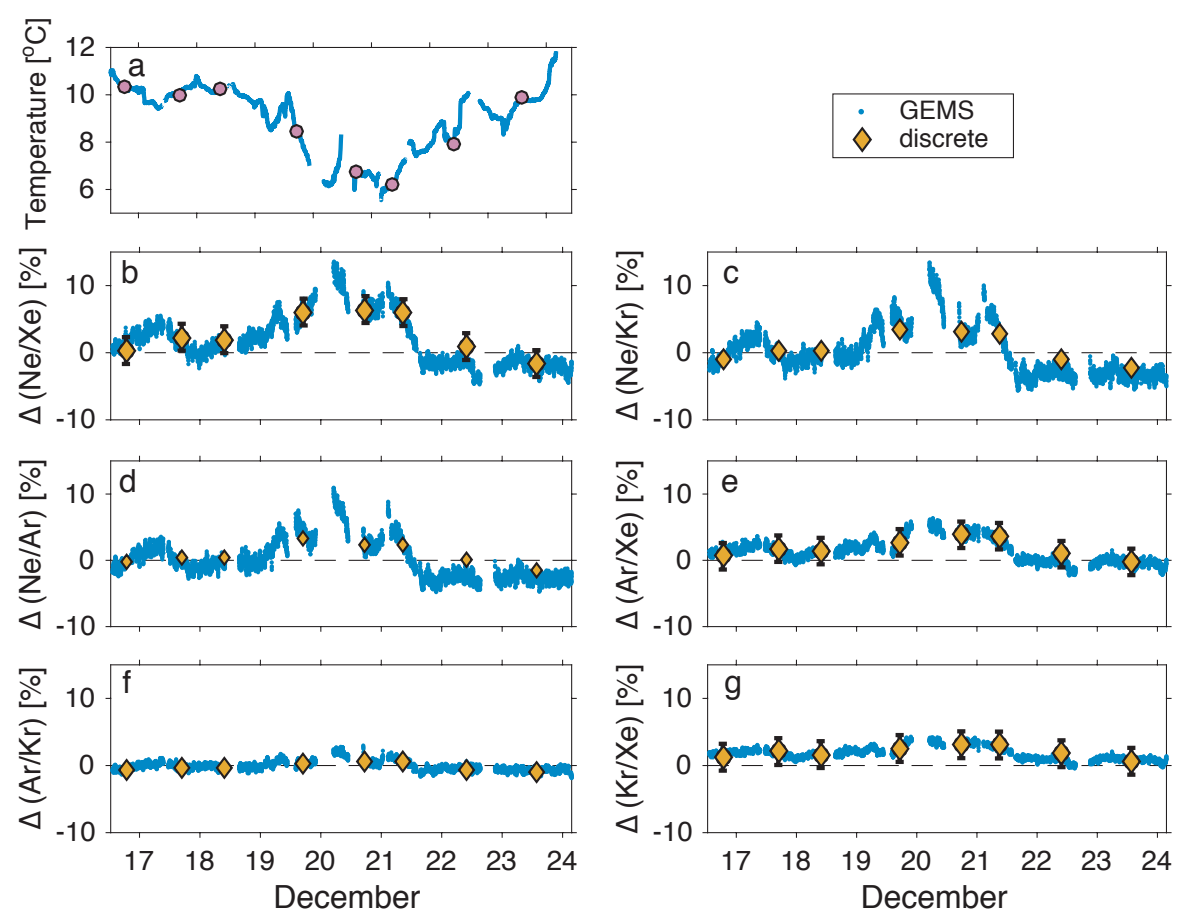

Figure A-4: Field data from GEMS and discrete samples. Data for $\mathrm{Ne} / \mathrm{Xe}, \mathrm{Ar} / \mathrm{Xe}$, and $\mathrm{Kr} / \mathrm{Xe}$ was calibrated using discrete samples as described in the main text. 
in the signal intensity, particularly for Ne and Xe, when the ship's speed rapidly increased. From discussions with staff at Hiden Analytical, we suspect that the slightly piezoelectric materials used to insulate the amplifiers in the mass spectrometer's electronics cause the instrument response to be sensitive to the high-frequency vibrations of the ship's motor. We do not believe the issue is associated with power fluctuations, since we connected the mass spectrometer to an uninterruptible power conditioning and supply unit (Eaton 9130). We also tested connecting the instrument to the ship's battery power, instead of the main power system, and it did not improve the noise in the data.

We replaced the hard plastic feet on the TPS-Compact with rubber vibration-isolating feet, and took care to keep power cables from leaning on the mass spectrometer. These changes reduced vibration-induced noise and improved the data quality; however, the data measured on both ships was still more noisy than data on a fixed platform (i.e., in the lab). Other investigators have used more robust vibration isolation apparatuses (e.g. pneumatic vibration isolation platforms) to successfully isolate mass spectrometers and optical instruments from vibrations on ships and aircraft,(Pratt et al., 2009; Dubé et al., 2006; Volpe et al., 2001) and such an apparatus could potentially be used for the GEMS.

We determined the accuracy of the GEMS by comparison with 12 discrete samples collected during a research cruise in Monterey Bay, CA on the R/V Western Flyer. The relative percent accuracy of the $\mathrm{Ne} / \mathrm{Xe}$ and $\mathrm{Ar} / \mathrm{Kr}$ ratios was 1.9 and $1.5 \%$, respectively. The remaining gas ratios had accuracies of 4.4-5.7\%. We suspect that the wide range in accuracy is partially related to the different amplifiers used to measure the different masses. $\mathrm{Ne}$ and Xe are the least abundant gases and are measured on the same amplifier (range $10^{-11}$ Torr), thus we expect them to be the most sensitive to, but affected similarly by vibrationinduced noise in the amplifier output. Ar was measured with an amplification range of $10^{-8}$ Torr (we measured ${ }^{36} \mathrm{Ar}$ in this experiment). Kr was measured with a range of $10^{-10}$ Torr. Therefore, Ar and Kr will be less sensitive to noise in the amplifiers, since the signal is greater and amplification is less. For gas ratios where one gas has a high amplification range setting $\left(10^{-11}\right.$ Torr $)$, and the other has a low amplification $\left(10^{-10}\right.$ to $10^{-8}$ Torr $)$, the measured ratio is less reliable. Therefore, $\mathrm{Ar} / \mathrm{Kr}$ is the most accurate ratio, and $\mathrm{Ne} / \mathrm{Xe}$ is the second most accurate ratio.

For applications on ships and other environments where the instrument may experience vibrations, we strongly recommend further testing of vibration-isolating equipment.

\section{A.6 Other potential applications}

In addition to the field experiment described in the main text, there are many other attractive applications for the GEMS. The GEMS could be used for lab-based tank experiments to quantify air-sea gas exchange fluxes under varying wind speeds (Mesarchaki et al., 2015a) 
and varying levels of ice cover (Lovely et al., 2015). For this type of study, the GEMS has clear advantages over discrete sampling: first, since the data is displayed in real time, the user would be able to ensure steady state gas distributions were established for all four noble gases at a specific set of forcing conditions, and then the forcing conditions could be changed. Second, the GEMS would enable many more measurements to be collected at a wide range of forcing conditions, without dramatically increasing the cost of the study, which would result in a more complete data set. Third, the water could be recirculated back to the tank after flowing through the membrane contactor, meaning that the sampling process would not perturb the water (or ice) level in the tank or change the total volume of water in the tank.

The GEMS could also be used for tank experiments aimed at characterizing the timescale and extent of gas partitioning between water and ice during freezing and melt. The continuous measurements and response time of 90-410 s would be effective for this type of study, whereas discrete gas sampling would provide less information (Loose et al., 2009b).

Furthermore, the GEMS could be used for introduced tracer studies to measure groundwater flow, glacial meltwater transport, and/or stream rearation (Benson et al., 2014; Visser et al., 2013). A tracer such as Xe would be added to the water and the GEMS would be installed downstream of the addition site. Since the GEMS operates continuously, unattended, users would be assured that high-frequency data is collected when the tracer passes through the sampling site, without the need for a worker sampling around the clock. The live display would ensure that the experiment would continue until all of the tracer had passed through the sampling site, and then be stopped.

The system could be used in tandem with sensors measuring bioactive gases, such as $\mathrm{CO}_{2}$ and $\mathrm{O}_{2}$, and other chemicals. Since the four noble gases we measure span a wide range in diffusivity, solubility, and dependence of solubility on temperature, they can be used to parameterize the physical processes controlling the distributions of other, non-inert gases and chemicals (Stanley and Jenkins, 2013). Thus, the GEMS could be used in marine and aquatic systems to improve determinations of net ecosystem metabolism using $\mathrm{O}_{2}$ measurements, net ecosystem calcification using $\mathrm{CO}_{2}$ and other carbonate parameters, and denitrification using $\mathrm{N}_{2}$ measurements. For many biologically active gases and chemicals, sensors capable of continuous measurements are already available, enabling the determination of daily cycles in biological fluxes. Thus, the GEMS enables determination of physical fluxes on the same time and spatial scales as the biological fluxes. As shown in the main manuscript, the GEMS can achieve the precision and accuracy that is needed for measurements in natural waters, where the noble gas concentrations are typically within $10 \%$ of equilibrium (Hamme and Severinghaus, 2007; Stanley et al., 2009b). 


\section{A.7 Parts list and photographs}

We provide tables of parts used, and photographs of the system, on the following pages.

Table A-3: Measurement components

\begin{tabular}{|c|c|c|}
\hline Description & Supplier & Part number \\
\hline $\begin{array}{l}\text { quadrupole mass spectrometer with triple mass filter, } \\
\text { Faraday and SCEM detectors, } 1-200 \mathrm{amu} \text { range }\end{array}$ & Hiden Analytical & $\begin{array}{l}\text { HAL 3F RC201, } \\
553010 / 200\end{array}$ \\
\hline quadrupole mass spectrometer ion source control module & Hiden Analytical & 304800 \\
\hline $\begin{array}{l}\text { direct source inlet flange for QMS } \\
\text { with } 1 / 4^{\prime \prime} \text { female VCR nut }\end{array}$ & Hiden Analytical & custom \\
\hline $\begin{array}{l}\text { vacuum pumping station (TPS-Compact with Turbo-V } 301 \\
\text { Navigator turbomolecular and IDP-3 dry scroll pump) }\end{array}$ & Agilent & $\mathrm{X} 3580 \mathrm{~A} \# 011 \# 024 \# 120$ \\
\hline vibration-isolating stud mount feet for TPS (order five) & MSC Industrial Supply & 36691178 \\
\hline full range pressure gauge kit for TPS-compact & Agilent & 9699201 \\
\hline manifold/housing for quadrupole & Sharon Vacuum & custom \\
\hline solid blank gasket for $4.5 " \mathrm{CF}$ flange, copper & Ideal Vacuum Products & P104352 \\
\hline $\begin{array}{l}\text { heating jacket, adjustable set-point, including controller } \\
\text { with communications and display module }\end{array}$ & MKS & custom, from series 48 \\
\hline power adapter for heating jacket & MKS & 48PWRCORD04 \\
\hline extension cable for heating jacket & MKS & 100014811 \\
\hline St2002 getter, cylindrical pieces, $6 \mathrm{~mm}$ diameter, $2 \mathrm{~mm}$ long & SAES Getters & $5 \mathrm{~F} 0542$ \\
\hline getter chamber & custom & See Figure A-8 \\
\hline manual valve, modified with capillary inlet & custom & See Figure A-8 \\
\hline manual $90^{\circ}$ valve between getters and mass spec inlet & Swagelok & SS-4BAG-V13-CU \\
\hline $\begin{array}{l}\text { heating element and shroud for getter can, } \\
1 \text { " diameter, } 4 \text { " long }\end{array}$ & Chromalox & MTB10E40 120V 750W \\
\hline $\begin{array}{l}\text { heating element and shroud for getter can, } \\
3 / 4 \text { " diameter, } 3 \text { " long }\end{array}$ & Chromalox & MTB7E30 120V 600W \\
\hline variac transformer (order two) & ISE, Inc. & 3PN1010B \\
\hline uninterruptible power supply (UPS) and power conditioner & Eaton & PW9130L-1000T-XL \\
\hline capillary ferrules for Swagelok fitting & Agilent & $5062-3508$ \\
\hline
\end{tabular}




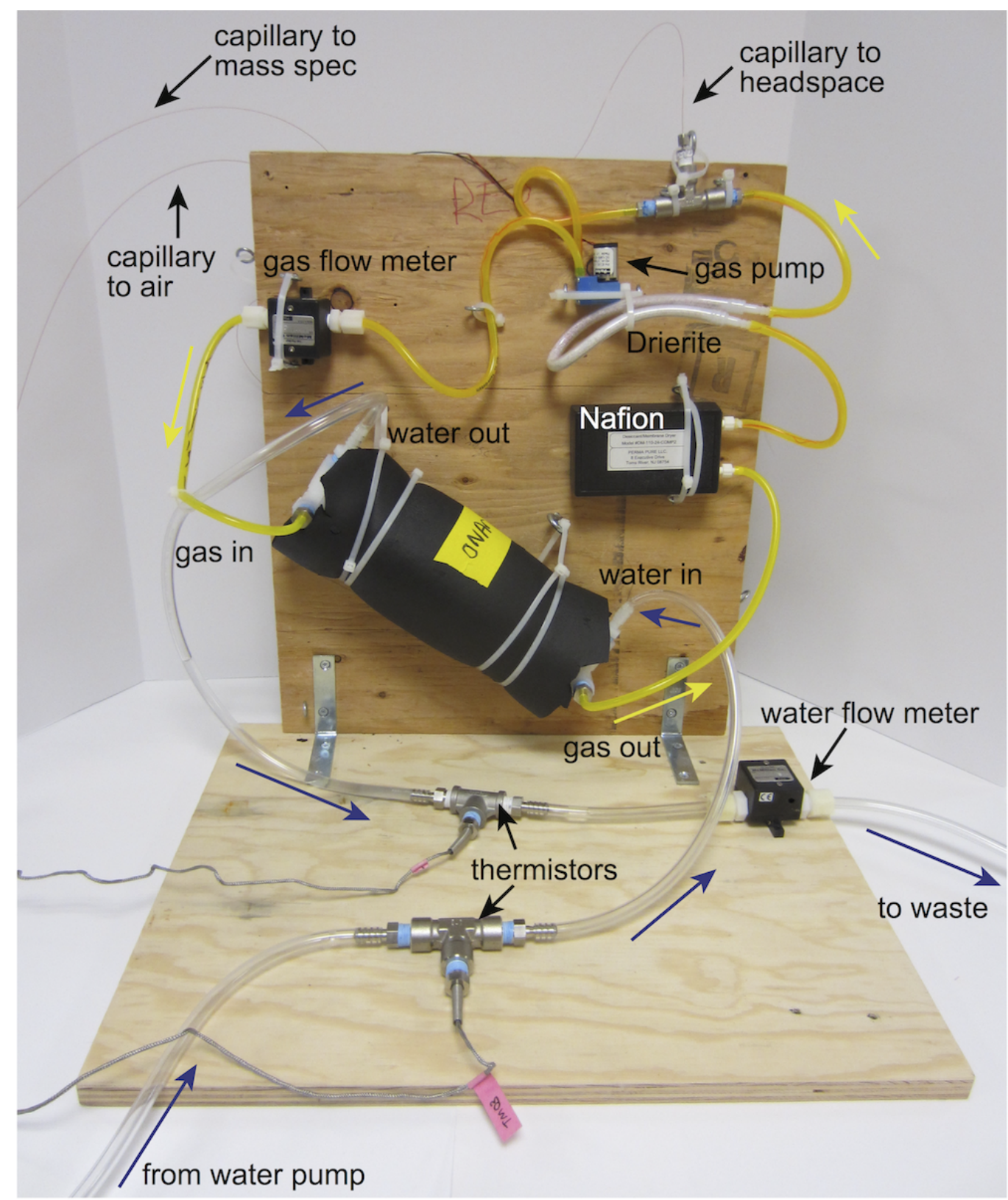

Figure A-5: The equilibration components (wet side) are attached to a board for organization. The board can be assembled before it is transported to the field site, to speed up the installation time. This photograph shows the front of the equilibration components board. The water flow direction is shown with blue arrows, and the gas flow direction is shown with yellow arrows. The water tubing is normally covered with foam insulation to reduce temperature change. The vertical board is $\sim 40 \mathrm{~cm}$ wide $\mathrm{x} 50 \mathrm{~cm}$ tall. 


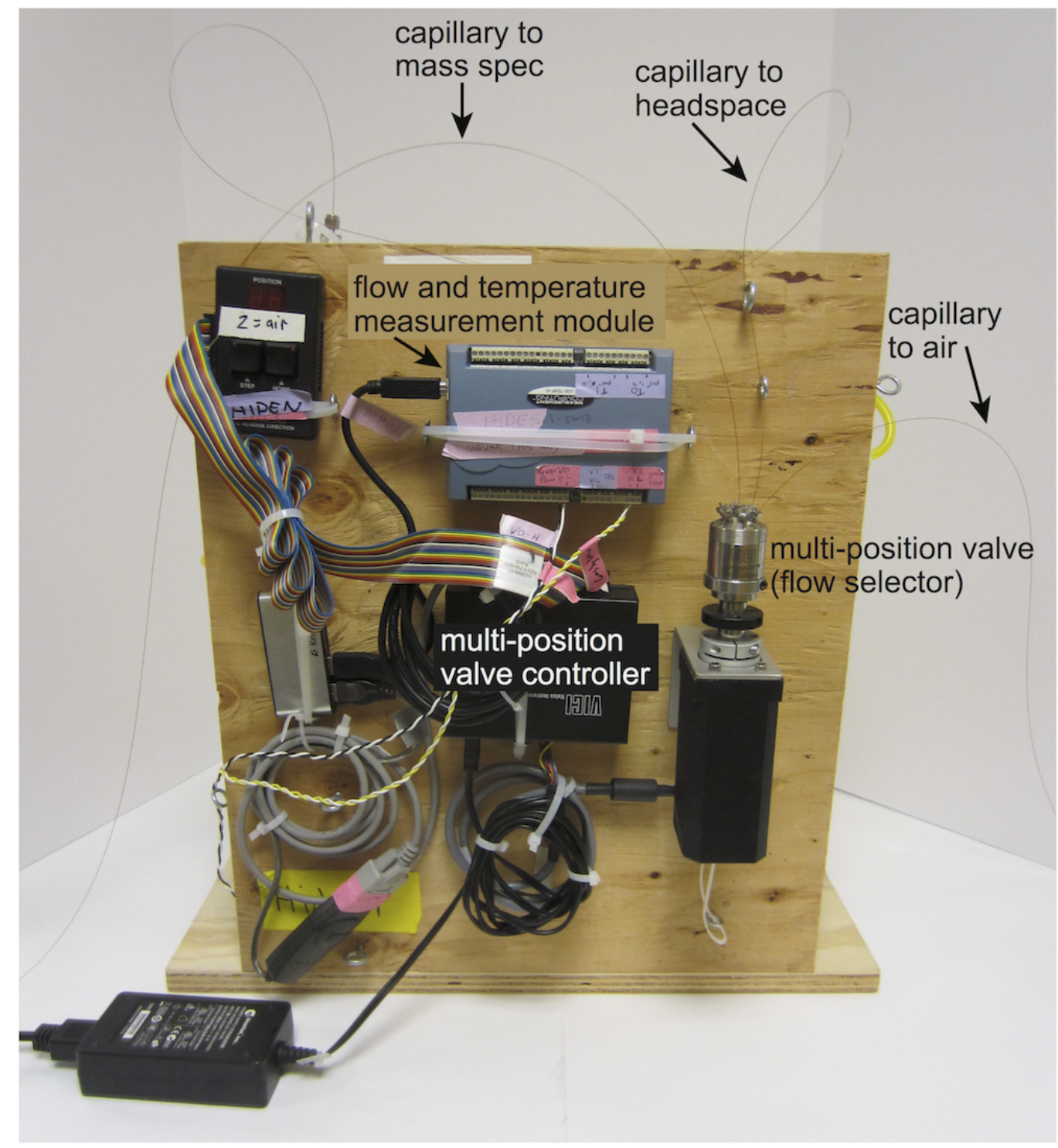

Figure A-6: The remainder of the equilibration components (wet side) of the GEMS. These components are on the opposite side of the board shown in Figure A-7. 


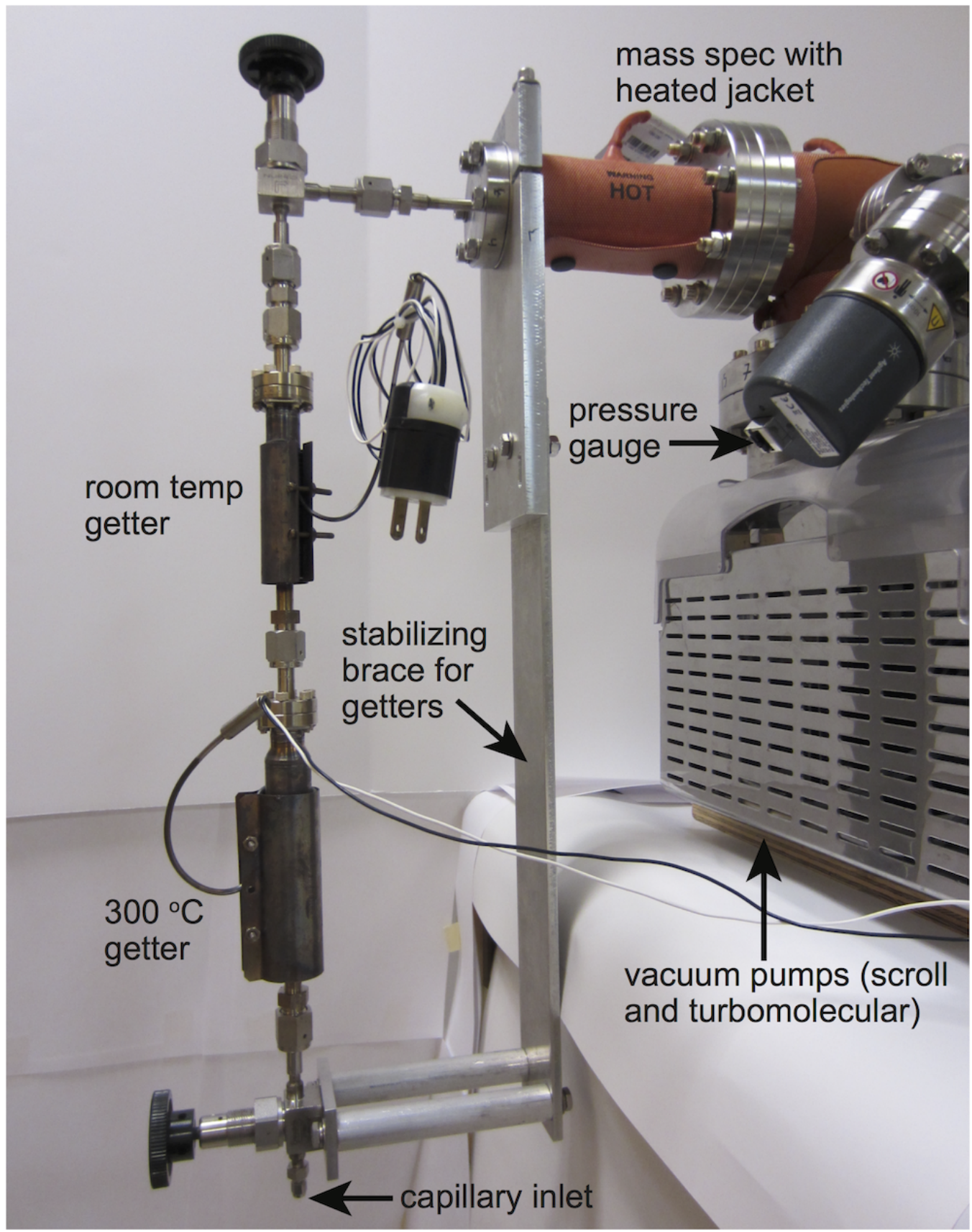

Figure A-7: The measurement components. See Figure A-8 for a schematic of the getters and valves. The combined apparatus (mass spec, pumps, and getter chambers) is $\sim 40 \mathrm{~cm}$ wide (perpendicular to the quadrupole), $85 \mathrm{~cm}$ long (parallel to the quadrupole), and $64 \mathrm{~cm}$ tall, from the capillary inlet to the top valve, including $\sim 51 \mathrm{~cm}$ tall above the counter and $13 \mathrm{~cm}$ below the counter. The total weight is $\sim 30 \mathrm{~kg}$. 


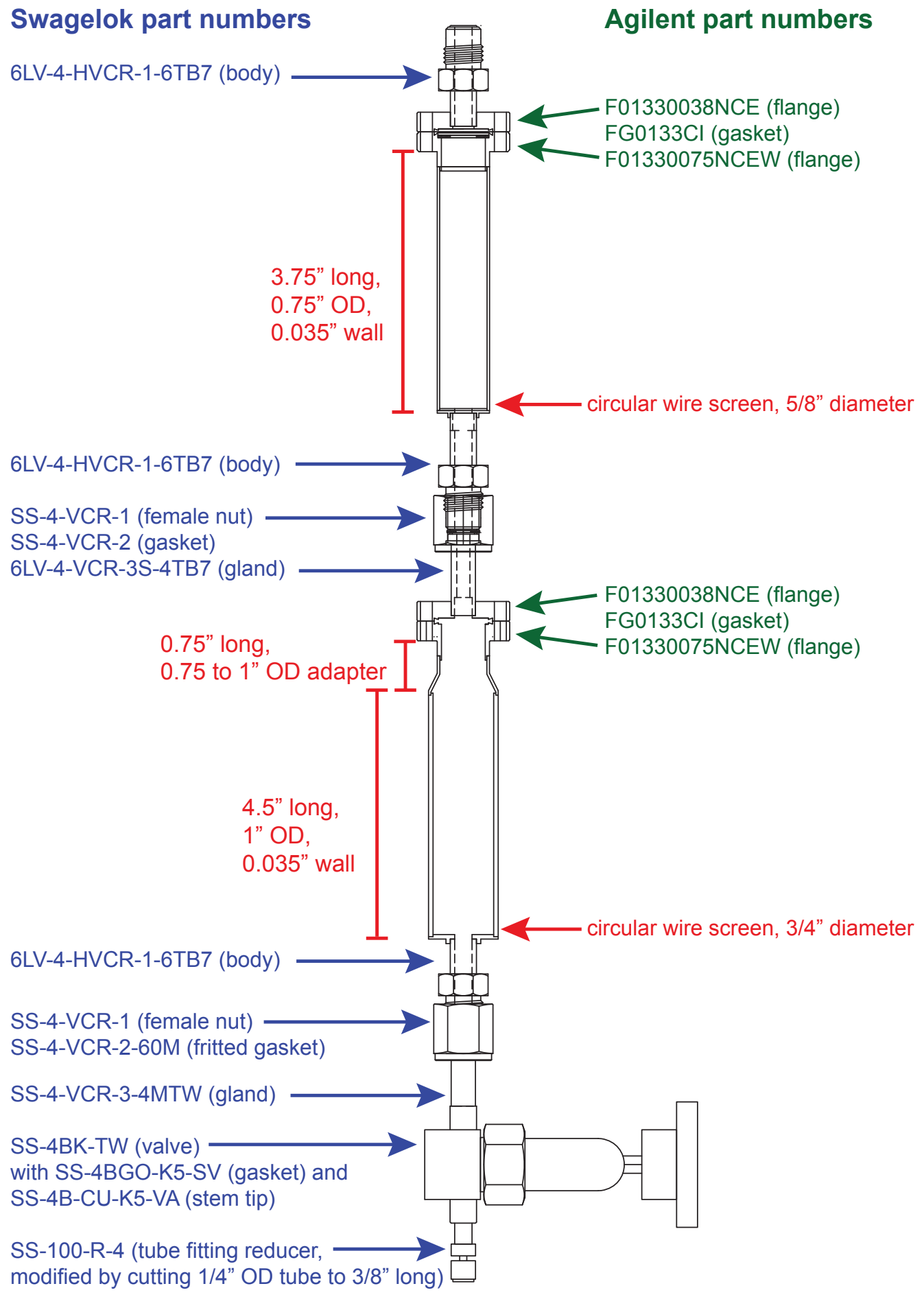

Figure A-8: Schematic of the getter cans, including dimensions and parts numbers. The parts are welded and leak-checked, the two getter cans are filled with getter, the flange connections are tightened, and then the heater jackets are attached so that the heating coils terminate at the bottom of each getter can. 
Table A-4: Equilibration components

\begin{tabular}{|c|c|c|}
\hline Description & Supplier & Part number \\
\hline membrane contactor, Extra-Flow $2.5 \times 8$, X40 fiber & Membrana Liqui-Cel & G420 \\
\hline $\begin{array}{l}\text { nylon hose barb to NPT adapters for } \\
\text { membrane contactor ports }\end{array}$ & McMaster-Carr & $\begin{array}{l}5372 \mathrm{~K} 112\left(1 / 4^{\prime \prime} \text { barb }\right) \\
5463 \mathrm{~K} 439\left(1 / 8^{\prime \prime} \text { barb }\right)\end{array}$ \\
\hline nafion dessicant box & PermaPure & DM-110-24 \\
\hline micro diaphragm pump for gas recirculation & Parker/Hargraves Fluidics & E191-11-060 \\
\hline patch cable to connect gas pump to power supply & McMaster-Carr & $6927 \mathrm{~K} 42$ \\
\hline water flow meter, $1-5 \mathrm{~L} / \mathrm{min}$ range, $3 / 8 "$ acetal fittings & McMillan & 101-8-D-A6 \\
\hline gas flow meter, $40-200 \mathrm{~mL} / \mathrm{min}$ range, $1 / 4 "$ acetal fittings & McMillan & 100-4-D-A4-G0 \\
\hline power supply for flow meters (order two) & McMillan & $110-00-08 \mathrm{~T}$ \\
\hline DC power supply with variable voltage & GW Instek & GPS-2303 \\
\hline water pump head, A-mount suction shoe, $1.17 \mathrm{~mL} / \mathrm{rev}$ & Cole-Parmer/Micropump & EW-07003-04 \\
\hline water pump drive, $150-4000 \mathrm{rpm}$ & Cole-Parmer/Micropump & EW-07003-90 \\
\hline $\begin{array}{l}\text { felt filter socks, 2-layer, } 5 \mu \mathrm{m} \text { inner, } 100 \mu \mathrm{m} \text { outer } \\
\text { pore size, } 12 \text { in long x } 1.5 \text { in wide }\end{array}$ & Universal Filters & PG5/100-1.5FX12 \\
\hline 10" filter canisters & Pentair/Pentek & 158643 \\
\hline pleated and/or string-wound filters $(100,20,5 \mu \mathrm{m})$ & Neo-Pure & PH-27097-100 \\
\hline deactivated fused silica capillary, $0.05 \mathrm{~mm}$ ID, $0.36 \mathrm{~mm}$ OD & Agilent & $160-2655-10$ \\
\hline capillary tubing cutter & Agilent & $5181-8836$ \\
\hline Valco multi-position valve with flow selector & VICI Valco & C5-1306EMH2Y \\
\hline ferrules for Valco valve & VICI Valco & FS1.36 or FS1.3PK-5 \\
\hline capillary adapter for sampling headspace in recirculation loop & Swagelok & SS-100-1-4 \\
\hline $\begin{array}{l}\text { t-shaped fitting for gas recirculation loop } \\
\text { and thermistors (order three) }\end{array}$ & Swagelok & SS-4-T \\
\hline $\begin{array}{l}\text { stainless steel } 1 / 4 \text { " NPT to } 1 / 8 \text { " hose barb adapter } \\
\text { for gas recirculation (order two) }\end{array}$ & Swagelok & SS-2-HC-1-4 \\
\hline $\begin{array}{l}\text { stainless steel } 1 / 4 " \text { NPT to } 1 / 4 " \text { hose barb adapter } \\
\text { for thermistors (order four) }\end{array}$ & Swagelok & SS-4-HC-1-4 \\
\hline flexible PVC tubing, $1 / 4 "$ ID x 3/8" OD (for water) & McMaster-Carr & $5233 \mathrm{~K} 56$ \\
\hline flexible PVC tubing, $1 / 8$ " ID x 1/4" OD (for gas) & McMaster-Carr & $5233 \mathrm{~K} 52$ \\
\hline foam rubber insulation for flexible PVC tubing & McMaster-Carr & $4463 \mathrm{~K} 131$ \\
\hline foam rubber insulation for membrane contactor & McMaster-Carr & $4463 \mathrm{~K} 145$ \\
\hline thermistors (order two) & OMEGA Engineering & TH-44032-1/4NPT \\
\hline thermocouples for air and heater jacket & OMEGA Engineering & 5TC-TT-T-24-72 \\
\hline thermocouples for getter chambers (order two) & OMEGA Engineering & KTSS-14U-12 \\
\hline multi-sensor temperature and voltage measurement device & Measurement Computing & USB-TEMP-AI \\
\hline thermocouple and voltage measurement device & Measurement Computing & USB-TC-AI \\
\hline
\end{tabular}




\section{Appendix B}

\section{Supplemental Information for Chapter 3:}

Quantifying air-sea gas exchange using noble gases in a coastal upwelling zone 


\section{B.1 Software and data}

The MATLAB code that was used in this paper for calculating air-sea fluxes with a variety of gas exchange parameterizations is available on Zenodo (Manning and Nicholson, 2016) and GitHub (http://www.github.com/dnicholson/gas_toolbox). The noble gas data is available as a supplement to the original publication (Manning et al., 2016c).

\section{B.2 Description of figures}

The gas data and model results in the main paper are presented using the gas solubilties of Lott and Jenkins (personal communication, 2015) for He, Kr, and Xe, and Hamme and Emerson (2004a) for Ne and Ar. Here, we present the gas data and model results using the published solubilities of Weiss (1971) for He, Weiss and Kyser (1978) for Kr, Hamme and Emerson (2004a) for Ne and Ar, and Wood and Caputi (1966) fit following the procedure of Hamme and Emerson (2004a) for Xe. We have provided a MATLAB function for calculating Xe solubility using Hamme's fit to Wood and Caputi (1966) in our toolbox of MATLAB functions (Manning and Nicholson, 2016). At the typical sea surface conditions during our study $\left(\mathrm{S}=34.4 \mathrm{PSS}, \mathrm{T}=16{ }^{\circ} \mathrm{C}\right.$ ), the solubilities of Lott and Jenkins are $2.2 \%$ higher for $\mathrm{He}, 1.3 \%$ higher for $\mathrm{Kr}$, and $0.1 \%$ less for Xe, compared to the published solubilities. 

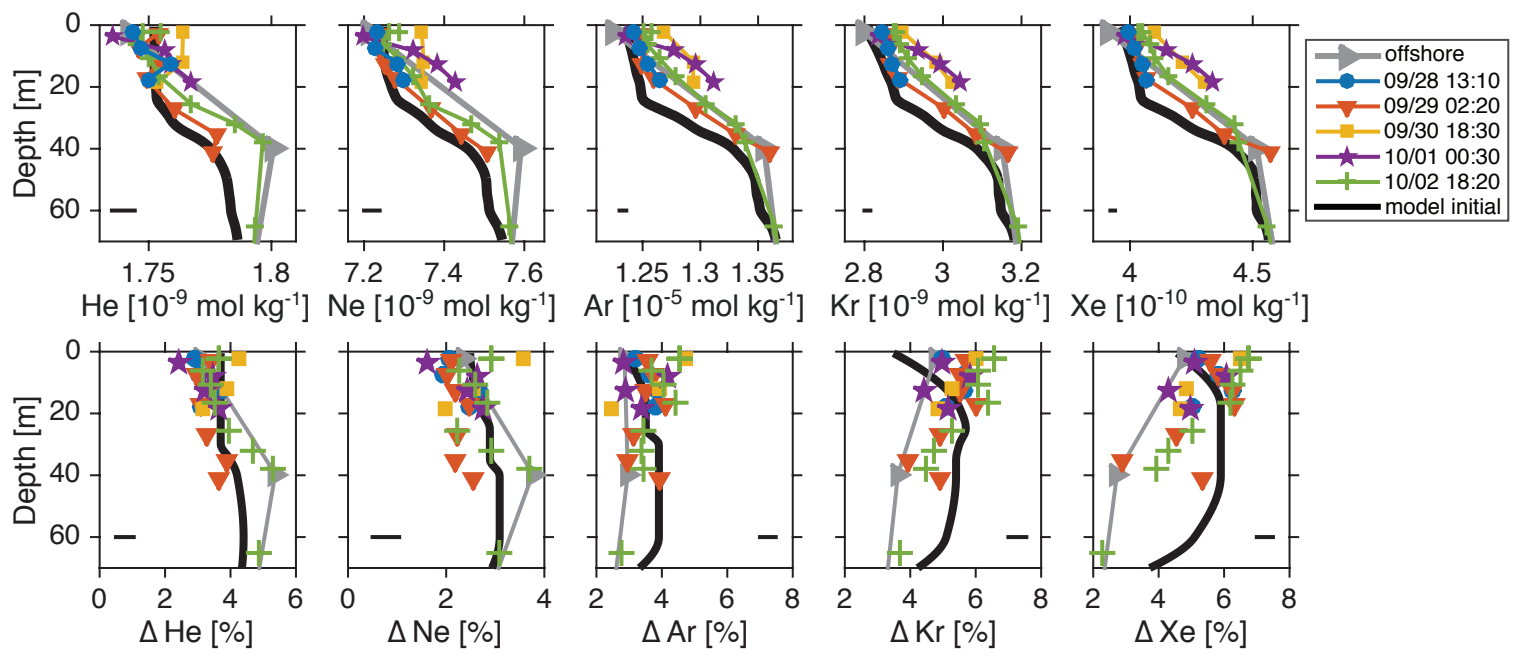

Figure B-1: Measured profiles and idealized initial profile of noble gas concentrations (a-e) and saturation anomalies $(\mathrm{f}-\mathrm{j})$ during the cruise. The offshore cast was collected on Sept 30, 21:30 and its location is shown in Figure 1 in the main text. The black line is the idealized profile that was used to initialize the model on Sept 28, 00:00. The horizontal black lines show the estimated measurement error. This figure is equivalent to Figure 3-2 in the main text, but uses published solubility functions for He, Kr, and Xe (Weiss, 1971; Weiss and Kyser, 1978; Wood and Caputi, 1966).
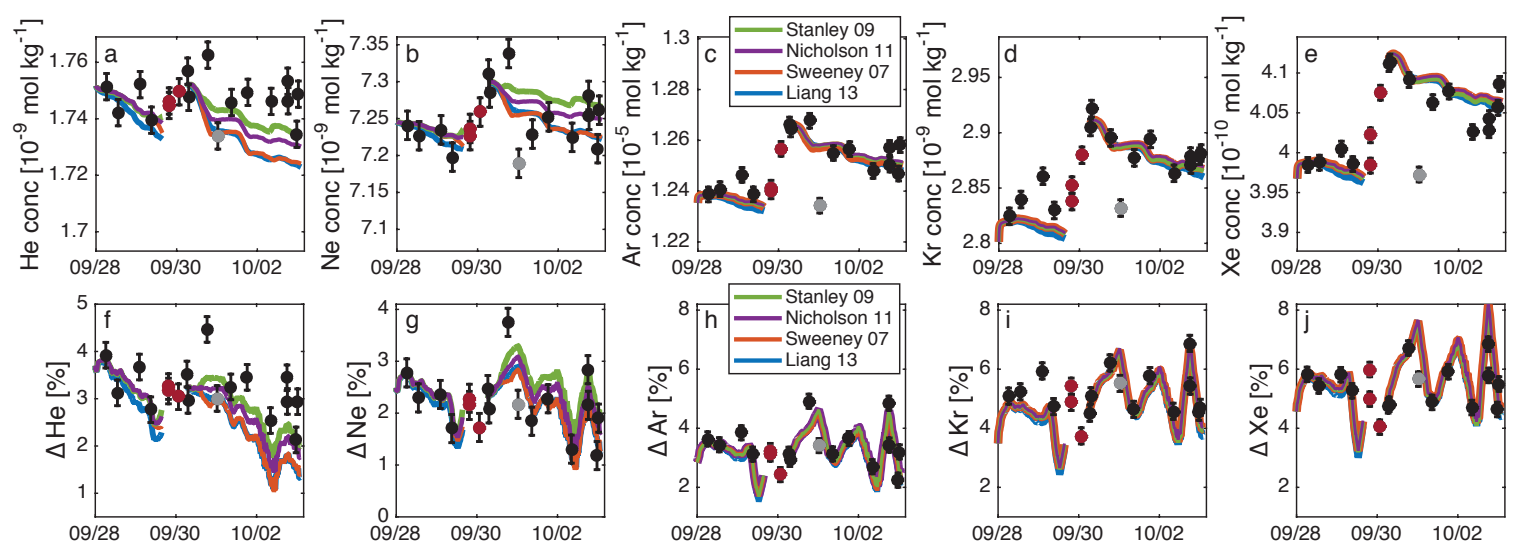

Figure B-2: Near-surface gas concentrations (a-e) and saturation anomalies $(\mathrm{f}-\mathrm{j}$ ), from samples and model results. The gap in the models around midnight Sept 30 corresponds to the upwelling event. Error bars reflect one standard deviation error in concentration measurement and do not include solubility uncertainty. Maroon circles are samples during the upwelling event that were not included in the Monte Carlo error analysis. The grey circle is an unexplained outlier that was included in the error analysis. This figure is equivalent to Figure 3-4 in the main text, but uses published solubility functions for $\mathrm{He}, \mathrm{Kr}$, and $\mathrm{Xe}$ (Weiss, 1971; Weiss and Kyser, 1978; Wood and Caputi, 1966). 

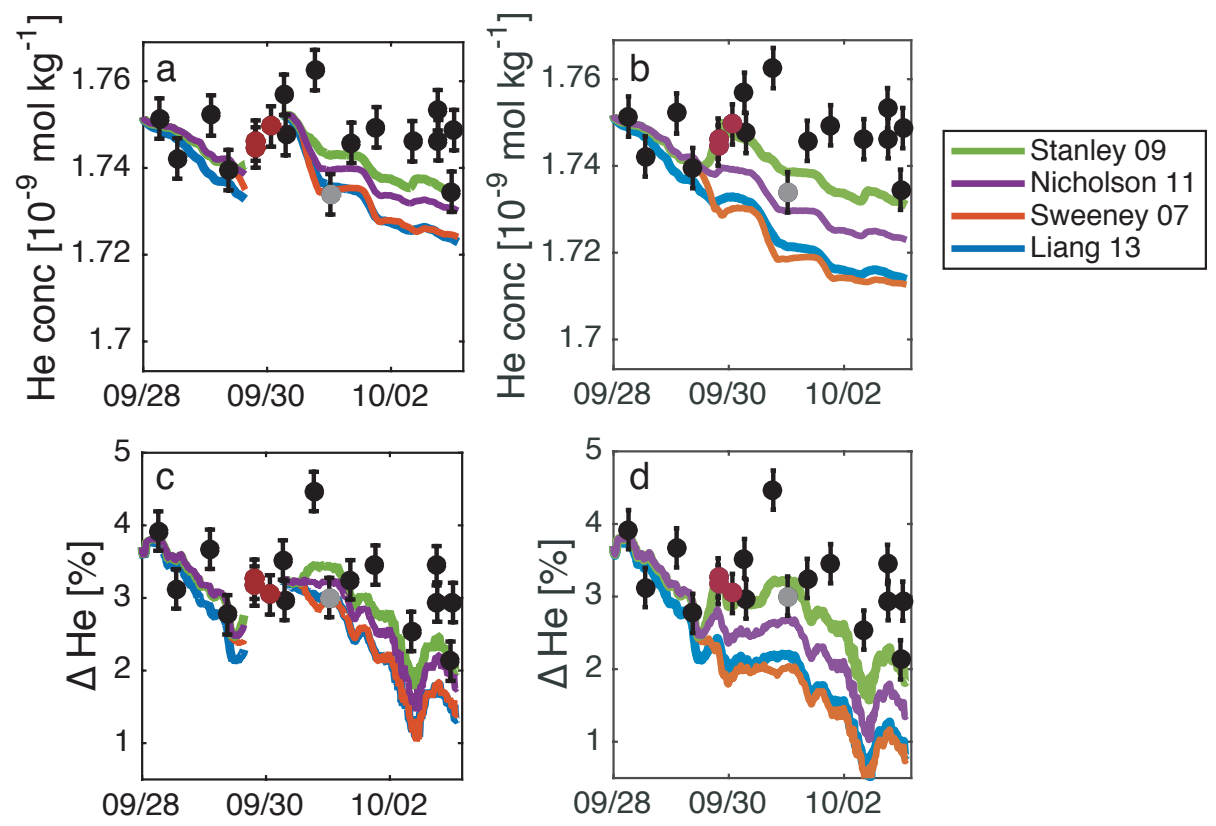

Figure B-3: Near-surface He concentrations ( $a-b)$ and He saturation anomalies $(c-d)$, from samples and model results, using the published He solubility of Weiss (1971). Comparison of model results with upwelling adjustment (a, c) and without upwelling adjustment (b, d). Note that all parameterizations underestimate the He concentrations and saturation anomalies. This figure is equivalent to Figure 3-4 (a, f) and Figure 3-5 (a, d) in the main text, but uses published solubility functions for He (Weiss, 1971; Weiss and Kyser, 1978; Wood and Caputi, 1966).
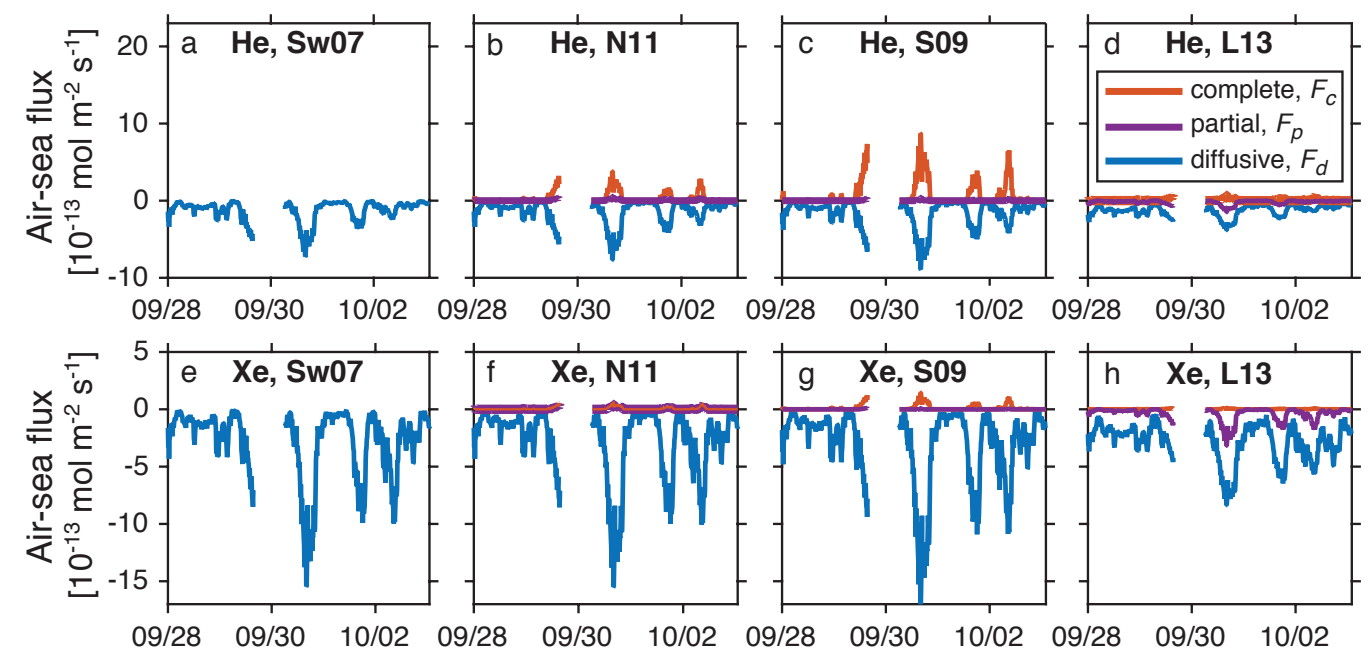

Figure B-4: Air-sea gas fluxes of He $(\mathrm{a}-\mathrm{d})$ and Xe $(\mathrm{e}-\mathrm{h})$ during the time series modeled using all four parameterizations. Positive fluxes are into the ocean. The flux is separated into three components: diffusive flux $\left(F_{d}\right)$, complete bubble trapping $\left(F_{c}\right)$, and partial bubble trapping $\left(F_{p}\right)$. The Sw07 parameterization only includes $F_{d}$. This figure is equivalent to Figure 3-6 in the main text, but uses published solubility functions for He and Xe (Weiss, 1971; Wood and Caputi, 1966). 


\section{Appendix C}

Supplemental Information for Chapter 4: Impact of recently upwelled water on productivity investigated using in situ and incubation-based methods in Monterey Bay 


\section{C.1 Introduction}

The supporting information contains figures showing maps of underway $\Delta\left(\mathrm{O}_{2} / \mathrm{Ar}\right)$, temperature, and chlorophyll data (Figures C-1-C-2) and additional calculations with $\mathrm{O}_{2} / \mathrm{Ar}$ : estimates of a time rate of change term for Phase 2 (Figure C-3) and model simulations of the evolution of mixed layer $\Delta\left(\mathrm{O}_{2} / \mathrm{Ar}\right)$ during Phase 2 in the recently upwelled water (Figure C-4). We also show the relationship between temperature, salinity, and $\mathrm{O}_{2} / \mathrm{Ar}$ (FigureC-5). Data will be available as a supplement to the published paper. Code for calculating gross oxygen production from triple oxygen isotope data is available at http://github.com/caramanning and will be provided as a supplement to the published paper. 


\section{C.2 Supplementary figures}
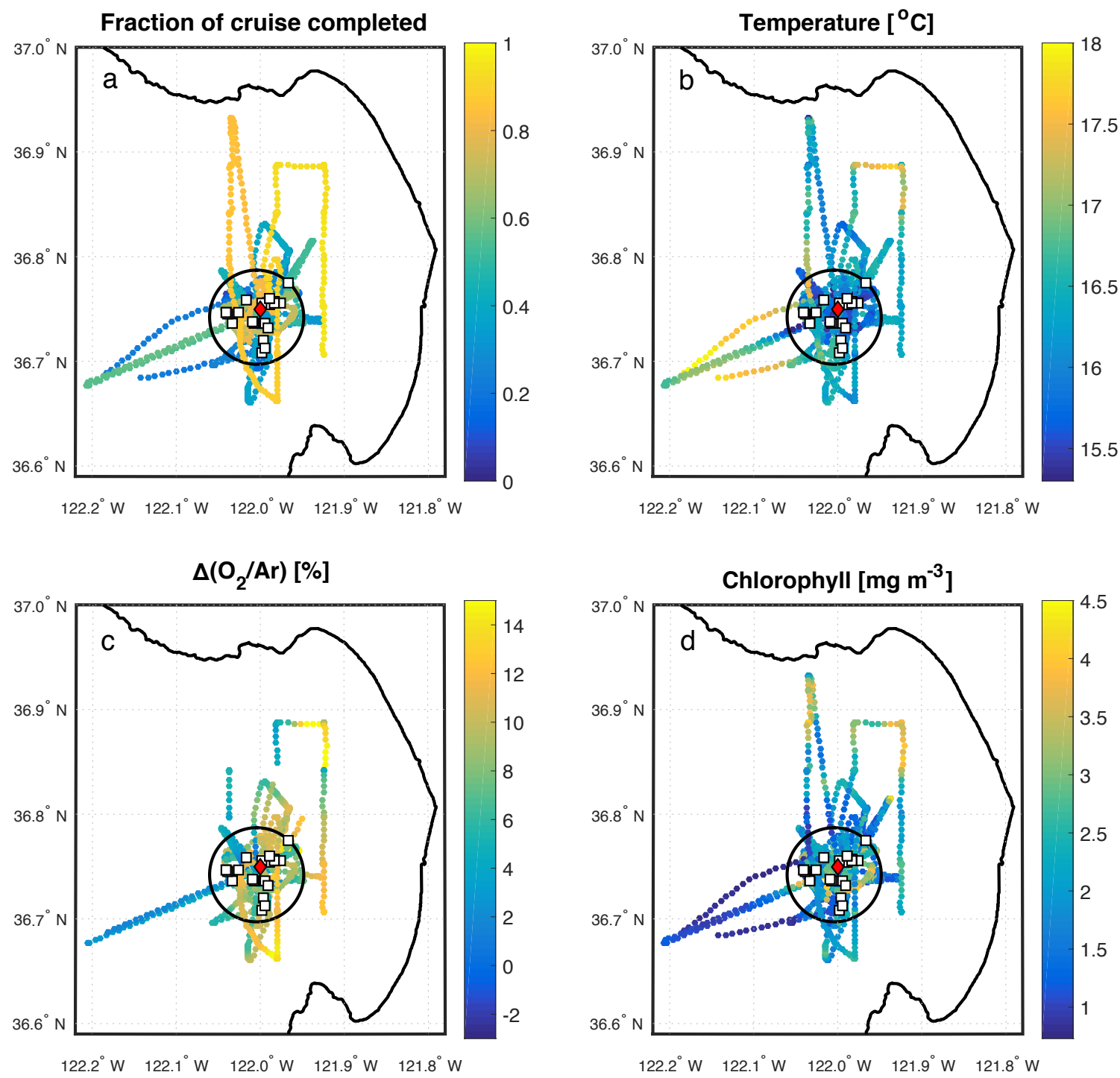

Figure C-1: Underway shipboard measurements showing all data. Plots are (a) fraction of cruise completed, (b) temperature, (c) $\Delta\left(\mathrm{O}_{2} / \mathrm{Ar}\right)$ and (d) chlorophyll. Gaps in $\Delta\left(\mathrm{O}_{2} / \mathrm{Ar}\right)$ data are due to air calibrations. The white squares show locations of CTD casts and red diamond shows the location of mooring M1. The black circle shows the main study area used in the paper (within $5 \mathrm{~km}$ of the mean CTD profile location). 

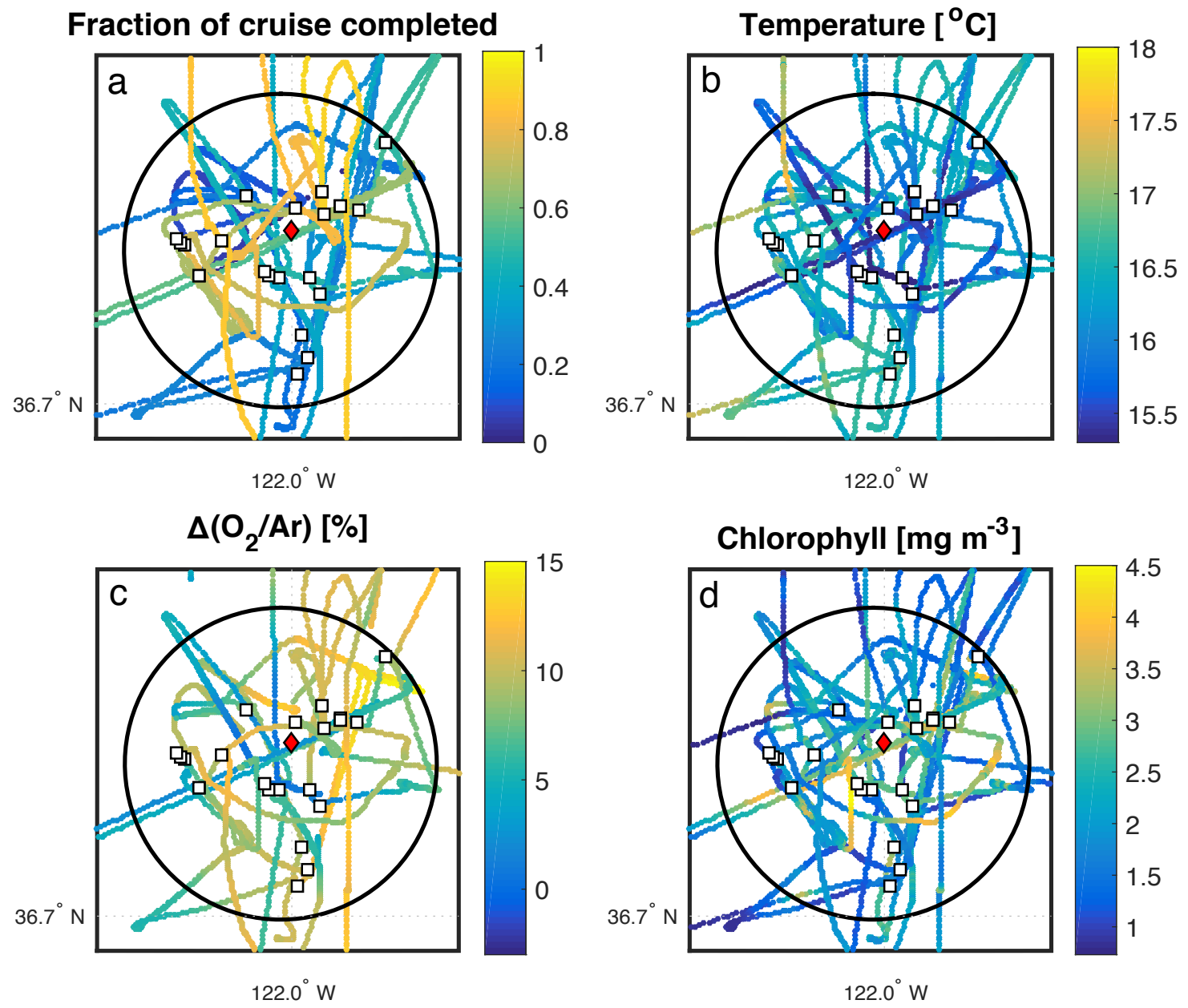

Figure C-2: Same as Figure C-1 but zoomed in on the main study area. Plots are (a) fraction of cruise completed, (b) temperature, (c) $\Delta\left(\mathrm{O}_{2} / \mathrm{Ar}\right)$ and (d) chlorophyll. Gaps in $\Delta\left(\mathrm{O}_{2} / \mathrm{Ar}\right)$ data are due to air calibrations. The white squares show locations of CTD casts and the red diamond shows the location of mooring M1. The black circle shows the main study area used in the paper (within $5 \mathrm{~km}$ of the mean CTD profile location). 

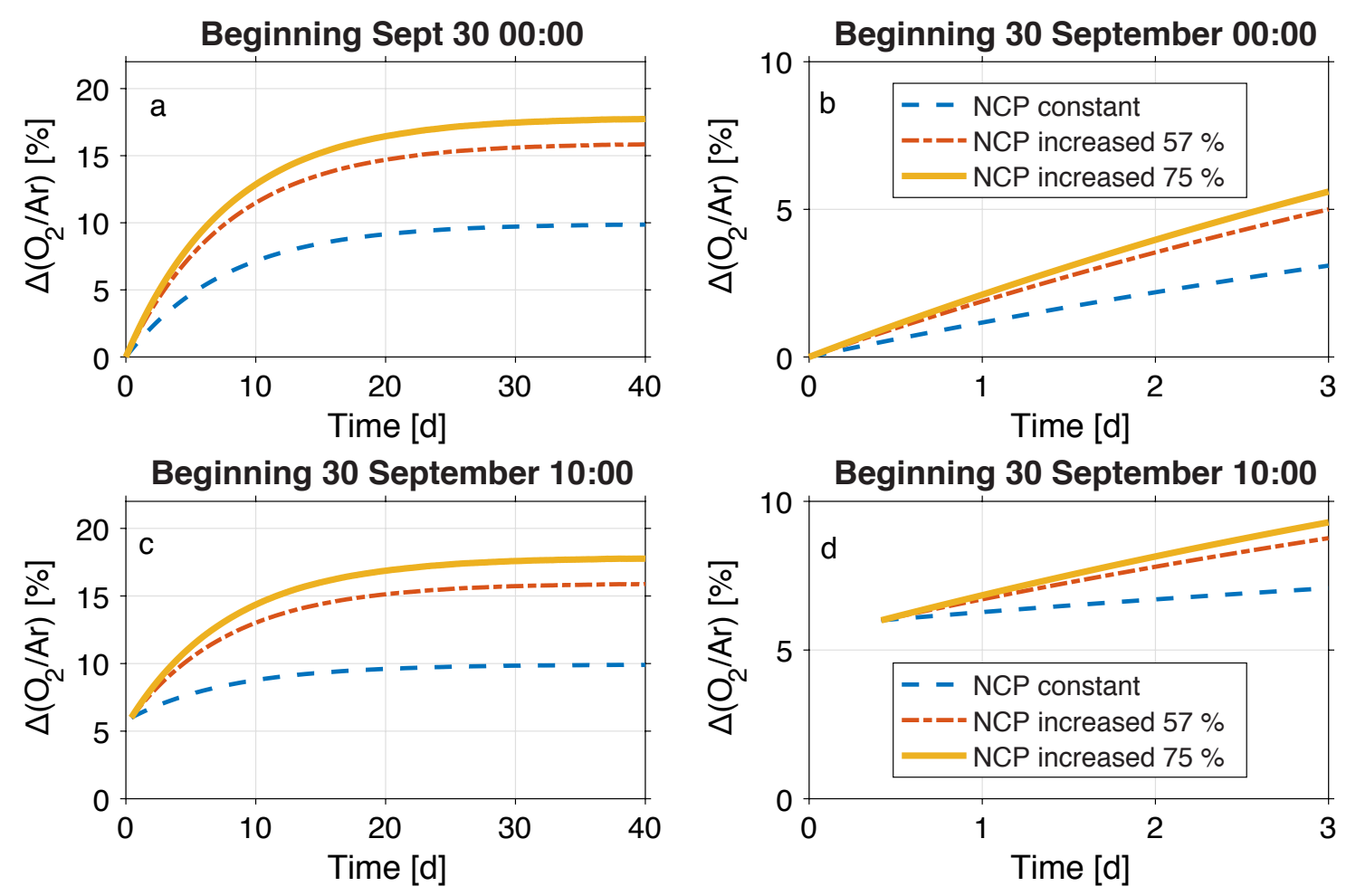

Figure C-3: Modeled change in $\Delta\left(\mathrm{O}_{2} / \mathrm{Ar}\right)$ following a perturbation (i.e. following the arrival of low- $\mathrm{O}_{2}$, recently upwelled water at the study site). The model is run with two different initial conditions: (a, b) $0 \%$ (the value on 30 September 00:00), and (c, d) $6 \%$ (the value on 30 September 10:00). Panels (a) and (c) show the evolution of $\Delta\left(\mathrm{O}_{2} / \mathrm{Ar}\right)$ toward steady state and panels $(\mathrm{b})$ and $(\mathrm{d})$ show the increase expected by the end of the cruise. The model uses a gas transfer velocity, $\mathrm{k}=1.8 \mathrm{~m} \mathrm{~d}^{-1}$, a mixed layer depth $=14 \mathrm{~m}$, and a vertical mixing flux of $3.5 \mathrm{mmol} \mathrm{O}_{2} \mathrm{~m}^{-2} \mathrm{~d}^{-1}$, consistent with the calculations in the main text. The constant NCP is $47 \mathrm{mmol} \mathrm{O} \mathrm{m}^{-2} \mathrm{~d}^{-1}$ (the rate in Phase 1 ), the $57 \%$ increase is equivalent to the increase in ${ }^{14} \mathrm{C}-\mathrm{PP}$ in Phase 2 relative to Phase 1 , and the $75 \%$ increase is equivalent to the increase in ${ }^{15} \mathrm{NO}_{3}^{-}$uptake $\left({ }^{15} \mathrm{~N}\right.$-new $\left.\mathrm{P}\right)$ in Phase 2 relative to Phase 1. 

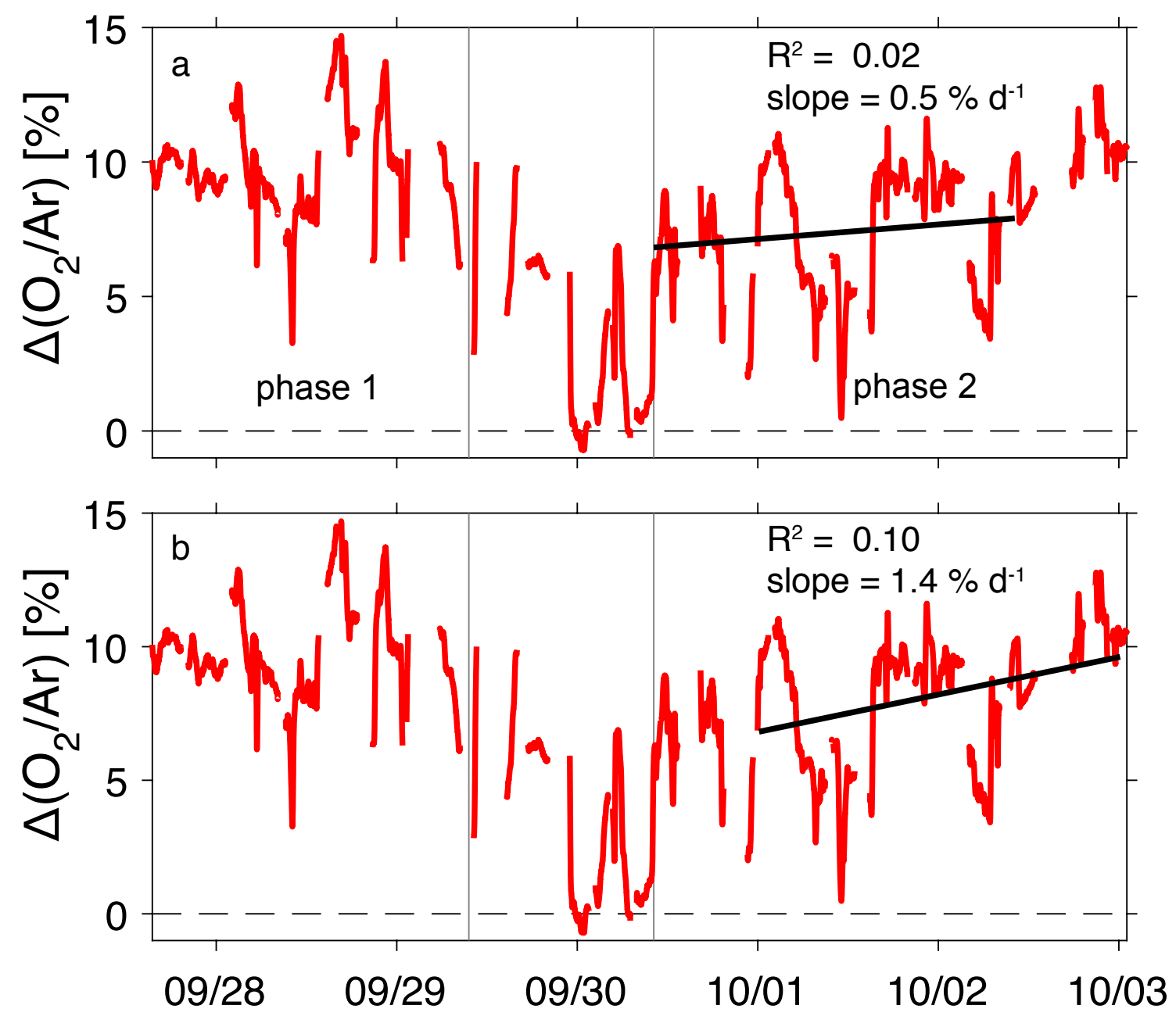

Figure C-4: Calculation of a linear regression of $\Delta\left(\mathrm{O}_{2} / \mathrm{Ar}\right)$ versus time in Phase 2. In (a) the slope is calculated between 30 September 10:10 and 2 October 10:10, and in (b) the slope is calculated from 1 October 00:10 to 3 October 00:10. All slopes calculated over 48-h periods during Phase 2 give low $R^{2}$ values (0.02 to 0.10) and the slope varies from 0.5 to 1.4 $\% \mathrm{~d}^{-1}$ depending on the start time. One contributor to the low $R^{2}$ values is likely diurnal changes in $\mathrm{O}_{2}$, but the lack of a consistent slope with time indicates that we would have low confidence in any term for the rate of change with time in $\Delta\left(\mathrm{O}_{2} / \mathrm{Ar}\right)$ applied to the Phase 2 data. 

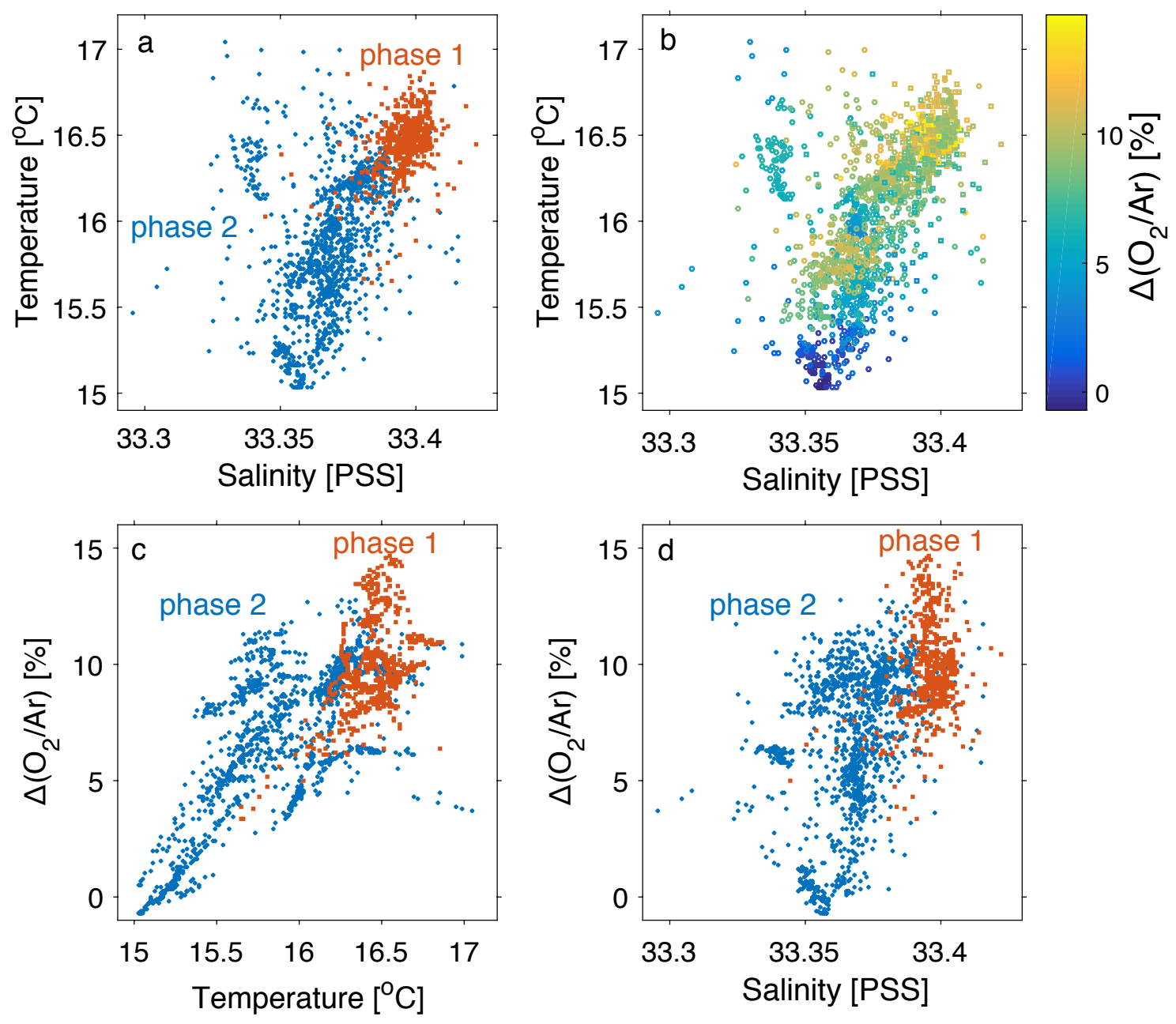

Figure C-5: Relationship between underway temperature, salinity, and $\Delta\left(\mathrm{O}_{2} / \mathrm{Ar}\right)$. Each data point is the average of $5 \mathrm{~min}$ of data. In all plots it is clear that the data cannot be easily separated into unique water masses. In (a), the majority of the points lie along a linear temperature-salinity mixing line connecting the waters of Phase 1 and Phase 2. In (c), deviations from the linear relationship between $\Delta\left(\mathrm{O}_{2} / \mathrm{Ar}\right)$ and temperature could result from either biological or physical processes. Panel (d) demonstrates that $\Delta\left(\mathrm{O}_{2} / \mathrm{Ar}\right)$ does not show a strong relationship with salinity. Similarly, in (b), $\Delta\left(\mathrm{O}_{2} / \mathrm{Ar}\right)$ is generally uncorrelated with temperature or salinity, however, the coldest waters have low $\Delta\left(\mathrm{O}_{2} / \mathrm{Ar}\right)$ and are reflective of the recently-upwelled water passing through the site on 29-30 September. 


\section{Appendix D}

Supplemental Information for Chapter 5: Changes in gross primary production, net community production, and air-water gas exchange during

seasonal ice melt in the Bras d'Or Lake, a Canadian estuary 


\section{D.1 ADCP data}
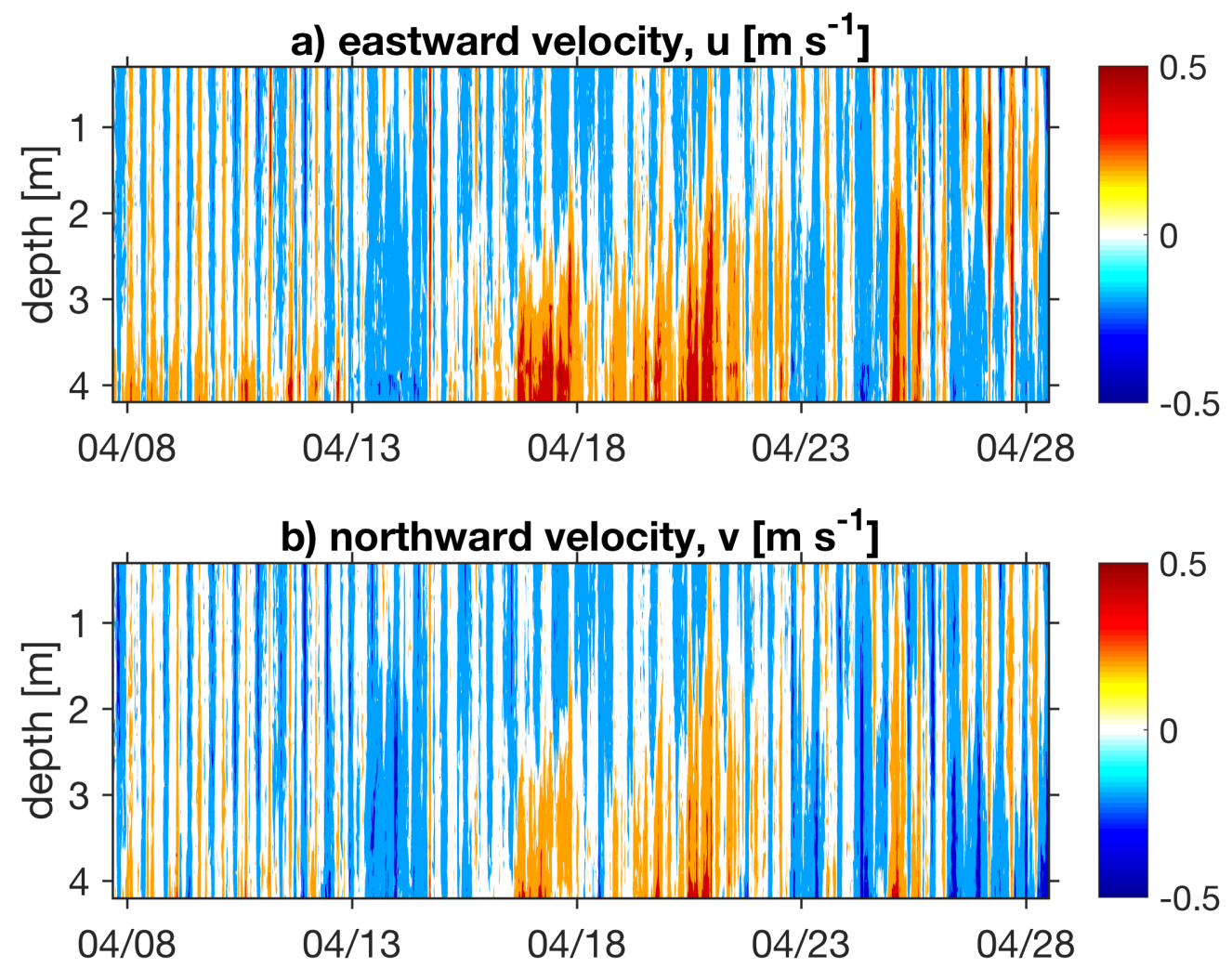

Figure D-1: ADCP measurements showing water velocity at the center of Little Narrows channel. The velocity data are 20-min averages, measured every $0.1 \mathrm{~m}$ from $0.3-4.2 \mathrm{~m}$ depth. 


\section{D.2 Photographs of study area}

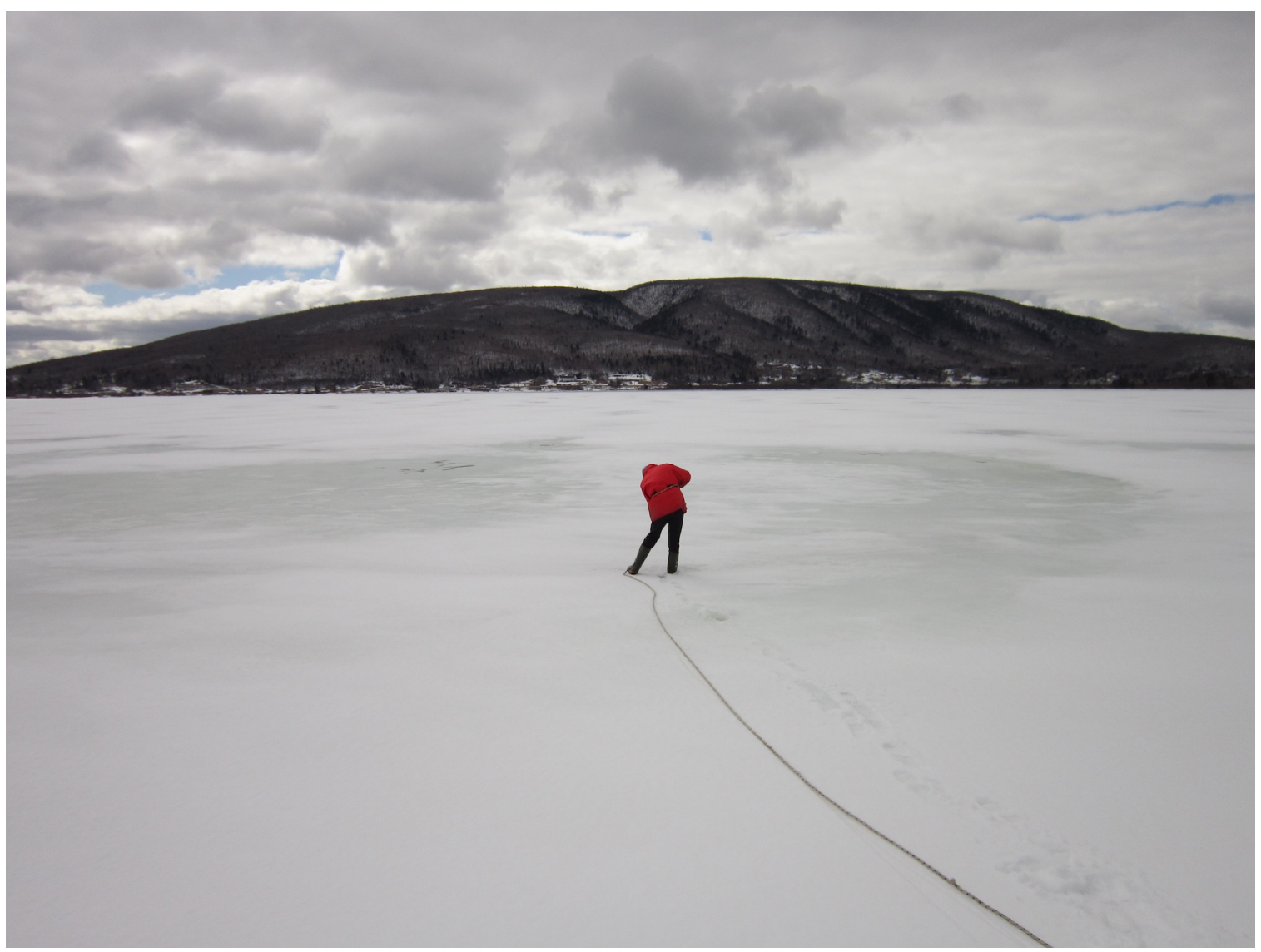

Figure D-2: Photograph of Whycocomagh Bay taken on 28 March 2013. 


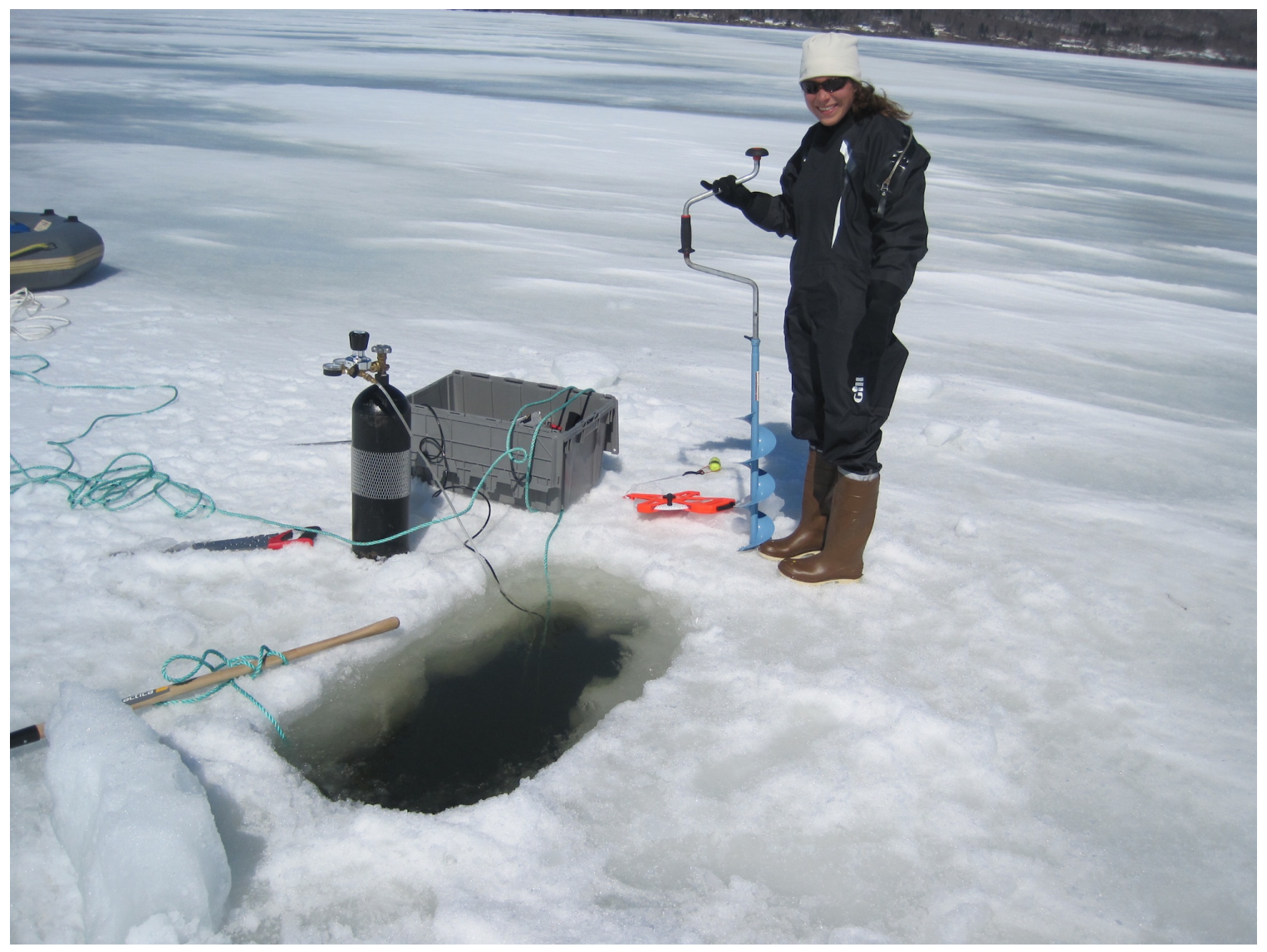

Figure D-3: Photograph of the tracer injection that occurred through ice on 30 March 2013. The ice behind the injection site shows some bare areas and/or melt ponds, which facilitate the transmittance of light through the ice to the water below. Photo taken by Brice Loose. 


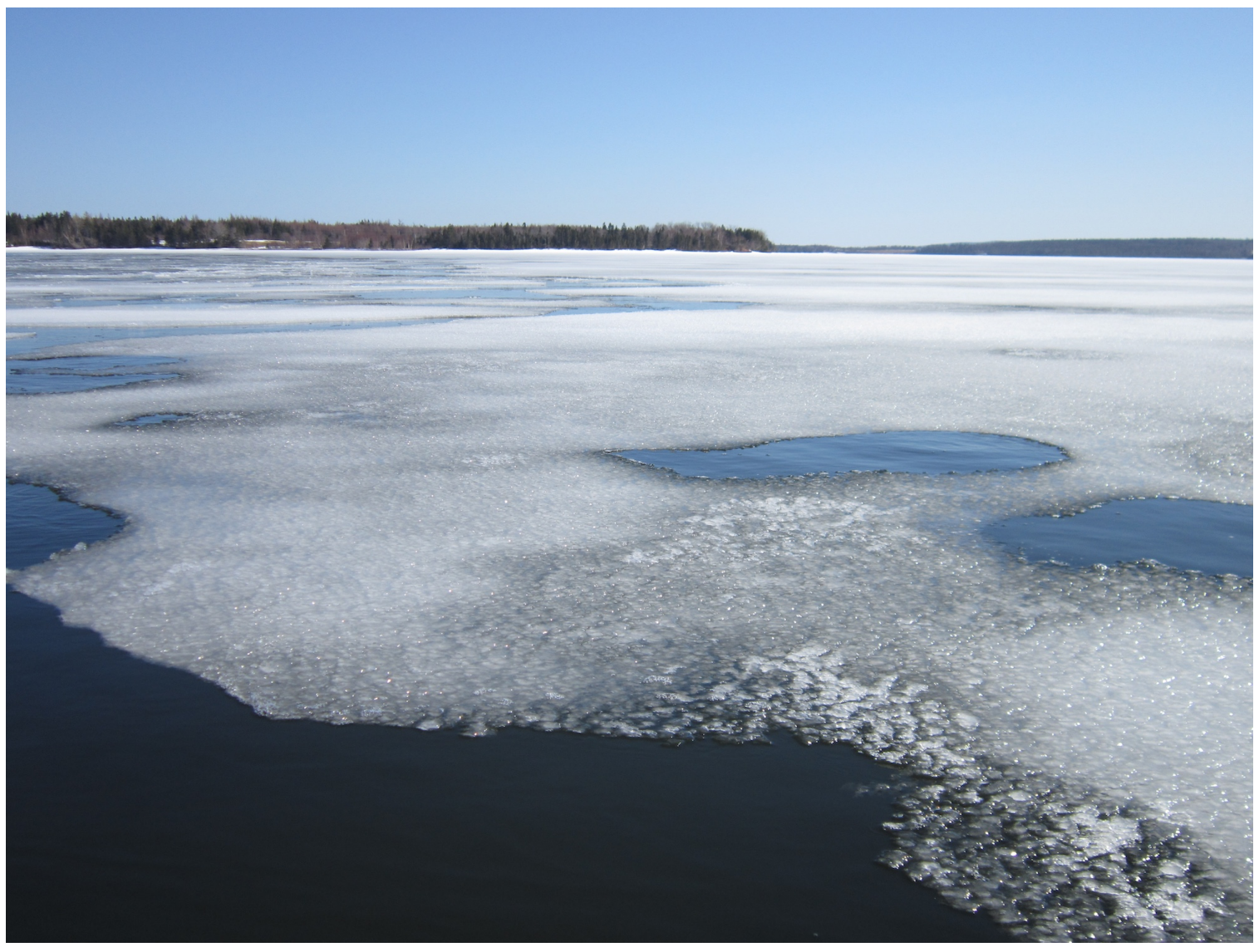

Figure D-4: Photograph of the ice edge in Whycocomagh Bay (close to Little Narrows) taken on 7 April 2013. 


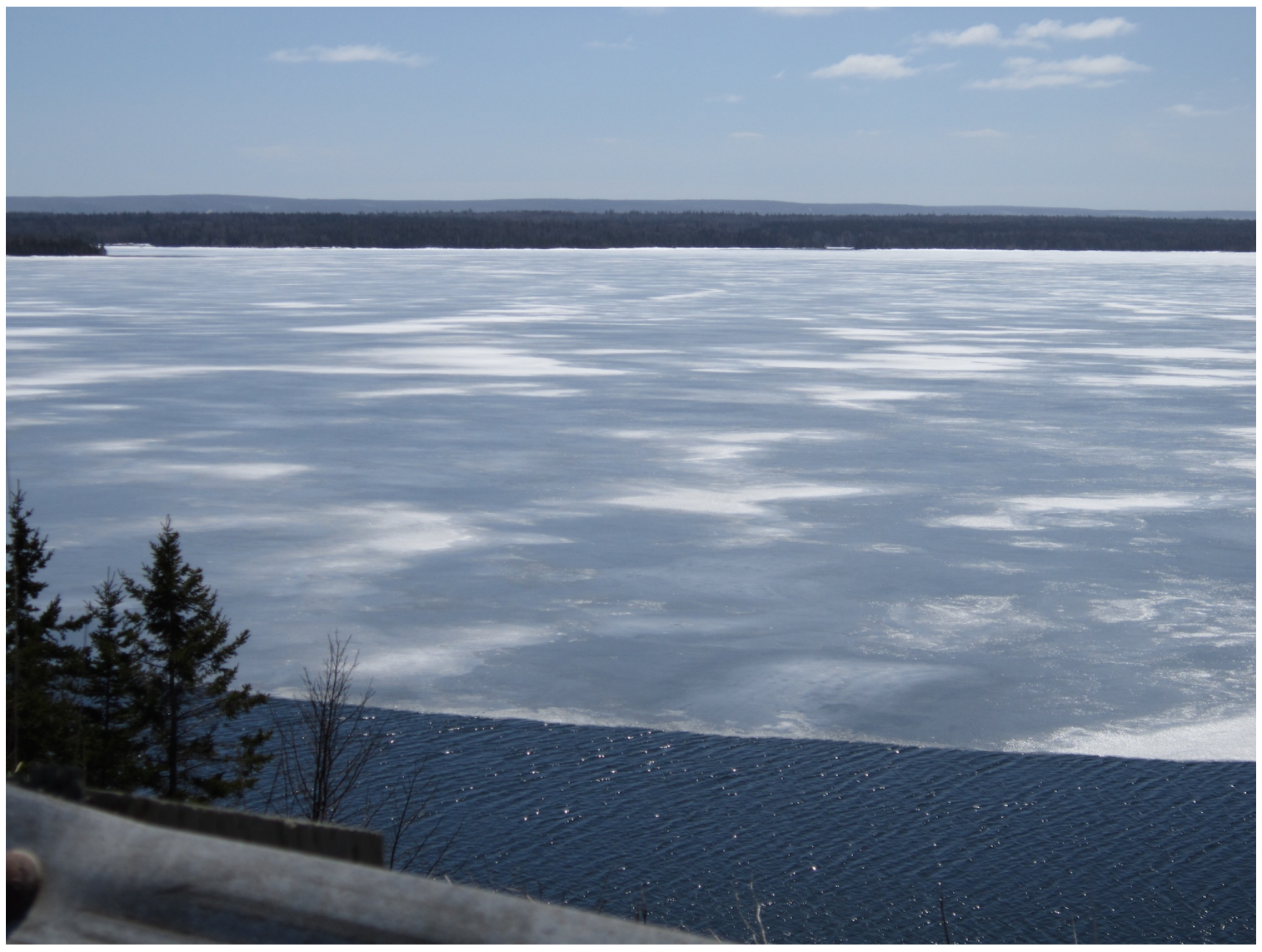

Figure D-5: Photograph of Whycocomagh Bay taken on 12 April 2013. The photo shows that the ice is thin, with melt ponds in some areas, and allows some light to pass through to the water below. 


\section{Bibliography}

ACIA. Impacts of a Warming Arctic-Arctic Climate Impact Assessment, volume 1. Cambridge University Press, Cambridge, UK, 2004.

Aeschbach, W. New perspectives for noble gases in oceanography. J. Geophys. Res. Oceans, 121(8):6550-6554, 2016. doi: 10.1002/2016JC012133.

Aeschbach-Hertig, W. and Solomon, D. Noble gas thermometry in groundwater hydrology. In The Noble Gases as Geochemical Tracers, pages 81-122. Springer, 2013.

Ahn, J., Headly, M., Wahlen, M., Brook, E. J., Mayewski, P. A., and Taylor, K. C. $\mathrm{CO}_{2}$ diffusion in polar ice: observations from naturally formed $\mathrm{CO}_{2}$ spikes in the Siple Dome (Antarctica) ice core. J. Glaciol., 54(187):685-695, 2008.

Aldrich, A. P., Jahme, M., and van den Berg, C. M. Antivibration electrode support for shipboard stripping voltammetry. Electroanal., 11(15):1155-1157, 1999.

Allen, C. B., Kanda, J., and Laws, E. A. New production and photosynthetic rates within and outside a cyclonic mesoscale eddy in the North Pacific subtropical gyre. Deep-Sea Res. Pt. I, 43(6):917-936, 1996.

Angert, A., Cappa, C. D., and DePaolo, D. J. Kinetic ${ }^{17} \mathrm{O}$ effects in the hydrologic cycle: Indirect evidence and implications. Geochim. Cosmochim. Acta, 68(17):3487-3495, 2004.

Aoki, N. and Makide, Y. The concentration of krypton in the atmosphere-its revision after half a century. Chem. Lett., 34(10):1396-1397, 2005.

Arrigo, K. R., Perovich, D. K., Pickart, R. S., Brown, Z. W., Van Dijken, G. L., Lowry, K. E., Mills, M. M., Palmer, M. A., Balch, W. M., Bahr, F., et al. Massive phytoplankton blooms under Arctic sea ice. Science, 336(6087):1408, 2012.

Asher, W. E., Karle, L. M., Higgins, B. J., Farley, P. J., Monahan, E. C., and Leifer, I. S. The influence of bubble plumes on air-seawater gas transfer velocities. J. Geophys. Res. Oceans, 101(C5):12027-12041, 1996.

Barkan, E. and Luz, B. The relationships among the three stable isotopes of oxygen in air, seawater and marine photosynthesis. Rapid Commun. Mass Spectrom., 25(16):2367-2369, 2011. doi: $10.1002 / \mathrm{rcm} .5125$.

Barkan, E. and Luz, B. High-precision measurements of ${ }^{17} \mathrm{O} /{ }^{16} \mathrm{O}$ and ${ }^{18} \mathrm{O} /{ }^{16} \mathrm{O}$ of $\mathrm{O}_{2}$ and $\mathrm{O}_{2} / \mathrm{Ar}$ ratio in air. Rapid Commun. Mass Spectrom., 17(24):2809-2814, 2003.

Barkan, E. and Luz, B. High precision measurements of ${ }^{17} \mathrm{O} /{ }^{16} \mathrm{O}$ and ${ }^{18} \mathrm{O} /{ }^{16} \mathrm{O}$ ratios in $\mathrm{H}_{2}$ O. Rapid Commun. Mass Spectrom., 19(24):3737-3742, 2005.

Barkan, E. and Luz, B. Diffusivity fractionations of $\mathrm{H}_{2}^{16} \mathrm{O} / \mathrm{H}_{2}^{17} \mathrm{O}$ and $\mathrm{H}_{2}^{16} \mathrm{O} / \mathrm{H}_{2}^{18} \mathrm{O}$ in air and their implications for isotope hydrology. Rapid Commun. Mass Spectrom., 21(18): 2999-3005, 2007. 
Bastviken, D., Sundgren, I., Natchimuthu, S., Reyier, H., and Gålfalk, M. Technical Note: Cost-efficient approaches to measure carbon dioxide $\left(\mathrm{CO}_{2}\right)$ fluxes and concentrations in terrestrial and aquatic environments using mini loggers. Biogeosciences, 12(12):3849-3859, 2015 .

Bates, N. R. and Mathis, J. T. The Arctic Ocean marine carbon cycle: evaluation of air-sea $\mathrm{CO}_{2}$ exchanges, ocean acidification impacts and potential feedbacks. Biogeosciences, 6 (11):2433-2459, 2009.

Bates, N. R., Moran, S. B., Hansell, D. A., and Mathis, J. T. An increasing $\mathrm{CO}_{2}$ sink in the Arctic Ocean due to sea-ice loss. Geophys. Res. Lett., 33(23), 2006. doi: 10.1029/ 2006GL027028. L23609.

Bauer, J. E., Cai, W.-J., Raymond, P. A., Bianchi, T. S., Hopkinson, C. S., and Regnier, P. A. The changing carbon cycle of the coastal ocean. Nature, 504(7478):61-70, 2013.

Beaird, N., Straneo, F., and Jenkins, W. Spreading of Greenland meltwaters in the ocean revealed by noble gases. Geophys. Res. Lett., 42(18):7705-7713, 2015.

Behrenfeld, M. J. and Falkowski, P. G. Photosynthetic rates derived from satellite-based chlorophyll concentration. Limnol. Oceanogr., 42(1):1-20, 1997.

Behrenfeld, M. J., Boss, E., Siegel, D. A., and Shea, D. M. Carbon-based ocean productivity and phytoplankton physiology from space. Global Biogeochem. Cy., 19(1), 2005. doi: 10.1029/2004GB002299. GB1006.

Bekryaev, R. V., Polyakov, I. V., and Alexeev, V. A. Role of polar amplification in longterm surface air temperature variations and modern Arctic warming. J. Climate, 23(14): 3888-3906, 2010.

Bender, M., Orchardo, J., Dickson, M.-L., Barber, R., and Lindley, S. In vitro $\mathrm{O}_{2}$ fluxes compared with ${ }^{14} \mathrm{C}$ production and other rate terms during the JGOFS Equatorial Pacific experiment. Deep-Sea Res. Pt. I, 46(4):637-654, 1999.

Benson, A., Zane, M., Becker, T. E., Visser, A., Uriostegui, S. H., DeRubeis, E., Moran, J. E., Esser, B. K., and Clark, J. F. Quantifying reaeration rates in alpine streams using deliberate gas tracer experiments. Water, 6(4):1013-1027, 2014.

Benson, B. B. and Krause, D. The concentration and isotopic fractionation of gases dissolved in freshwater in equilibrium with the atmosphere. 1. Oxygen. Limnol. Oceanogr., 25(4): 662-671, 1980a.

Benson, B. B. and Krause, D. Isotopic fractionation of helium during solution: A probe for the liquid state. J. Solution Chem., 9(12):895-909, 1980b.

Benson, B. B. and Krause, D. The concentration and isotopic fractionation of oxygen dissolved in freshwater and seawater in equilibrium with the atmosphere. Limnol. Oceanogr., 29(3):620-632, 1984.

Benson, B. B. and Krause Jr, D. Empirical laws for dilute aqueous solutions of nonpolar gases. J. Chem. Phys., 64(2):689-709, 1976. 
Berman, E. S. F., Levin, N. E., Landais, A., Li, S., and Owano, T. Measurement of $\delta^{18} \mathrm{O}, \delta^{17} \mathrm{O}$, and ${ }^{17} \mathrm{O}$-excess in water by off-axis integrated cavity output spectroscopy and isotope ratio mass spectrometry. Anal. Chem., 85(21):10392-10398, 2013.

Bertilsson, S., Burgin, A., Carey, C. C., Fey, S. B., Grossart, H.-P., Grubisic, L. M., Jones, I. D., Kirillin, G., Lennon, J. T., Shade, A., et al. The under-ice microbiome of seasonally frozen lakes. Limnol. Oceanogr., 58(6):1998-2012, 2013.

Beyerle, U., Aeschbach-Hertig, W., Imboden, D. M., Baur, H., Graf, T., and Kipfer, R. A mass spectrometric system for the analysis of noble gases and tritium from water samples. Environ. Sci. Technol., 34(10):2042-2050, 2000. doi: 10.1021/es990840h.

Bigg, G. R. and Rohling, E. J. An oxygen isotope data set for marine waters. J. Geophys. Res. Oceans, 105(C4):8527-8535, 2000. doi: 10.1029/2000JC900005.

Bittig, H. C. and Körtzinger, A. Tackling oxygen optode drift: Near-surface and in-air oxygen optode measurements on a float provide an accurate in situ reference. J. Atmos. Ocean. Tech., 32(8):1536-1543, 2015.

Blomquist, B. W., Huebert, B. J., Fairall, C. W., Bariteau, L., Edson, J. B., Hare, J. E., and McGillis, W. R. Advances in air-sea $\mathrm{CO}_{2}$ flux measurement by eddy correlation. Boundary-Layer Meteorol., 152(3):245-276, 2014. doi: 10.1007/s10546-014-9926-2.

Boetius, A., Albrecht, S., Bakker, K., Bienhold, C., Felden, J., Fernández-Méndez, M., Hendricks, S., Katlein, C., Lalande, C., Krumpen, T., et al. Export of algal biomass from the melting Arctic sea ice. Science, 339(6126):1430-1432, 2013.

Bond, N. A., Cronin, M. F., Freeland, H., and Mantua, N. Causes and impacts of the 2014 warm anomaly in the NE Pacific. Geophys. Res. Lett., 42(9):3414-3420, 2015.

Bondarenko, N. A., Timoshkin, O. A., Röpstorf, P., and Melnik, N. G. The under-ice and bottom periods in the life cycle of Aulacoseira baicalensis (K. Meyer) Simonsen, a principal Lake Baikal alga. Hydrobiologia, 568(1):107-109, 2006.

Bowen, G. J. Isoscapes: spatial pattern in isotopic biogeochemistry. Ann. Rev. Earth Pl. Sci., 38:161-187, 2010.

Bowen, G. J. and Good, S. P. Incorporating water isoscapes in hydrological and water resource investigations. WIREs Water, 2(2):107-119, 2015.

Brennwald, M. S., Hofer, M., and Kipfer, R. Simultaneous analysis of noble gases, sulfur hexafluoride, and other dissolved gases in water. Environ. Sci. Technol., 47(15):8599-8608, 2013a.

Brennwald, M. S., Vogel, N., Figura, S., Vollmer, M. K., Langenfelds, R., Steele, L. P., Maden, C., and Kipfer, R. Concentrations and isotope ratios of helium and other noble gases in the earth's atmosphere during 1978-2011. Earth Planet. Sci. Lett., 366:27-37, 2013b.

Bronk, D. A., Glibert, P. M., and Ward, B. B. Nitrogen uptake, dissolved organic nitrogen release, and new production. Science, 265(5180):1843-1846, 1994. 
Buesseler, K. O. Do upper-ocean sediment traps provide an accurate record of particle flux? Nature, 353(6343):420-423, 1991.

Bullister, J. L. Atmospheric Histories (1765-2015) for CFC-11, CFC-12, CFC-113, $\mathrm{CCl}_{4}$, $\mathrm{SF}_{6}$ and $\mathrm{N}_{2} \mathrm{O}$. NDP-095(2015). http://cdiac.ornl.gov/ftp/oceans/CFC_ATM_Hist/ CFC_ATM_Hist_2015, 2015.

Bullister, J. L., Wisegarver, D. P., and Menzia, F. A. The solubility of sulfur hexafluoride in water and seawater. Deep-Sea Res. Pt. I, 49(1):175-187, 2002.

Bullister, J. L., Wisegarver, D. P., and Sonnerup, R. E. Sulfur hexafluoride as a transient tracer in the North Pacific Ocean. Geophys. Res. Lett., 33(18), 2006. doi: 10.1029/ 2006GL026514. L18603.

Burd, A. B., Buchan, A., Church, M., Landry, M., McDonnell, A., Passow, U., Steinberg, D., and Benway, H. Towards a transformative understanding of the biology of the ocean's biological pump: Priorities for future research. Report of the NSF Biology of the Biological Pump Workshop, February 19-20, 2016 (Hyatt Place New Orleans, New Orleans, LA), 2016. doi: $10.1575 / 1912 / 8263$.

Bushinsky, S. M. and Emerson, S. A method for in-situ calibration of Aanderaa oxygen sensors on surface moorings. Mar. Chem., 155:22-28, 2013.

Bushinsky, S. M., Emerson, S. R., Riser, S. C., and Swift, D. D. Accurate oxygen measurements on modified Argo floats using in situ air calibrations. Limnol. Oceanogr. Methods, 2016. doi: 10.1002/lom3.10107.

Butterworth, B. J. and Miller, S. D. Air-sea exchange of carbon dioxide in the Southern Ocean and Antarctic marginal ice zone. Geophys. Res. Lett., 43(13):7223-7230, 2016. doi: 10.1002/2016GL069581. 2016GL069581.

Cahill, J. E. and Tracy, D. H. Effects of permeation of helium through the walls of fused silica capillary GC columns. J. High Res. Chromatogr., 21(10):531-539, 1998.

Cai, W.-J. Estuarine and coastal ocean carbon paradox: $\mathrm{CO}_{2}$ sinks or sites of terrestrial carbon incineration? Ann. Rev. Mar. Sci., 3:123-145, 2011.

Cai, W.-J., Dai, M., and Wang, Y. Air-sea exchange of carbon dioxide in ocean margins: A province-based synthesis. Geophys. Res. Lett., 33(12), 2006.

Cai, W.-J., Chen, L., Chen, B., Gao, Z., Lee, S. H., Chen, J., Pierrot, D., Sullivan, K., Wang, Y., $\mathrm{Hu}$, X., et al. Decrease in the $\mathrm{CO}_{2}$ uptake capacity in an ice-free Arctic Ocean basin. Science, 329(5991):556-559, 2010.

Caissie, D. and Robichaud, S. Towards a better understanding of the natural flow regimes and streamflow characteristics of rivers of the Maritime Provinces. Technical Report 2843, Fisheries and Oceans Canada, 2009.

Callaghan, A. H., Stokes, M. D., and Deane, G. B. The effect of water temperature on air entrainment, bubble plumes, and surface foam in a laboratory breaking-wave analog. $J$. Geophys. Res. Oceans, 119(11):7463-7482, 2014. doi: 10.1002/2014JC010351. 
Callies, J., Ferrari, R., Klymak, J. M., and Gula, J. Seasonality in submesoscale turbulence. Nature Commun., 6:6862, 2015. doi: 10.1038/ncomms7862.

Carlson, C. A., Ducklow, H. W., Michaels, A. F., et al. Annual flux of dissolved organic carbon from the euphotic zone in the northwestern Sargasso Sea. Nature, 371(6496): 405-408, 1994.

Carpenter, J. H. The Chesapeake Bay Institute technique for the Winkler dissolved oxygen method. Limnol. and Oceanogr., 10(1):141-143, 1965.

Carr, M.-E., Friedrichs, M. A. M., Schmeltz, M., Aita, M. N., Antoine, D., Arrigo, K. R., Asanuma, I., Aumont, O., Barber, R., Behrenfeld, M., et al. A comparison of global estimates of marine primary production from ocean color. Deep-Sea Res. Pt. II, 53(5): 741-770, 2006.

Cassar, N., Barnett, B. A., Bender, M. L., Kaiser, J., Hamme, R. C., and Tilbrook, B. Continuous high-frequency dissolved $\mathrm{O}_{2} / \mathrm{Ar}$ measurements by equilibrator inlet mass spectrometry. Anal. Chem., 81(5):1855-1864, 2009.

Cassar, N., DiFiore, P. J., Barnett, B. A., Bender, M. L., Bowie, A. R., Tilbrook, B., Petrou, K., Westwood, K. J., Wright, S. W., and Lefevre, D. The influence of iron and light on net community production in the Subantarctic and Polar Frontal Zones. Biogeosciences, $8(2): 227-237,2011$.

Cassar, N., Nevison, C. D., and Manizza, M. Correcting oceanic $\mathrm{O}_{2} /$ Ar-net community production estimates for vertical mixing using $\mathrm{N}_{2} \mathrm{O}$ observations. Geophys. Res. Lett., 41 (24):8961-8970, 2014.

Castro, M. C., Goblet, P., Ledoux, E., Violette, S., and Marsily, G. Noble gases as natural tracers of water circulation in the Paris Basin: 2. Calibration of a groundwater flow model using noble gas isotope data. Water Resour. Res., 34(10):2467-2483, 1998.

Castro-Morales, K. and Kaiser, J. Using dissolved oxygen concentrations to determine mixed layer depths in the Bellingshausen Sea. Ocean Sci., 8(1):1-10, 2012.

Cayan, D. R. Latent and sensible heat flux anomalies over the northern oceans: Driving the sea surface temperature. J. Phys. Oceanogr., 22(8):859-881, 1992.

Chang, B. X., Devol, A. H., and Emerson, S. R. Fixed nitrogen loss from the eastern tropical North Pacific and Arabian Sea oxygen deficient zones determined from measurements of $\mathrm{N}_{2}$ :Ar. Global Biogeochem. Cy., 26(3), 2012.

Ciais, P., Sabine, C., Bala, G., Bopp, L., Brovkin, V., Canadell, J., Chhabra, A., DeFries, R., Galloway, J., Heimann, M., Jones, C., Quéré, C. L., Myneni, R., Piao, S., and Thornton, P. Carbon and other biogeochemical cycles. In Fagerberg, J., Mowery, D. C., and Nelson, R. R., editors, Climate Change 2013: The Physical Science Basis. Contribution of Working Group I to the Fifth Assessment Report of the Intergovernmental Panel on Climate Change, chapter 10, pages 266-290. Cambridge University Press, Cambridge, United Kingdom and New York, NY, USA, 2013.

Clark, D. R., Flynn, K. J., and Owens, N. J. The large capacity for dark nitrate-assimilation in diatoms may overcome nitrate limitation of growth. New Phytol., 155(1):101-108, 2002. 
Clark, J. F., Schlosser, P., Wanninkhof, R., Simpson, H. J., Schuster, W. S., and Ho, D. T. Gas transfer velocities for $\mathrm{SF}_{6}$ and ${ }^{3} \mathrm{He}$ in a small pond at low wind speeds. Geophys. Res. Lett., 22(2):93-96, 1995.

Cloern, J. E. Our evolving conceptual model of the coastal eutrophication problem. Mar. Ecol. Prog. Ser., 210:223-253, 2001.

COESA working group. US Standard Atmosphere, 1976, 1976. National Aeronautics and Space Administration. U.S. Government Printing Office, Washington, D.C.

Cole, J. J. and Caraco, N. F. Atmospheric exchange of carbon dioxide in a low-wind oligotrophic lake measured by the addition of $\mathrm{SF}_{6}$. Limnol. Oceanogr., 43(4):647-656, 1998.

Comiso, J. C., Parkinson, C. L., Gersten, R., and Stock, L. Accelerated decline in the Arctic sea ice cover. Geophys. Res. Lett., 35(1), 2008.

Cota, G. F., Legendre, L., Gosselin, M., and Ingram, R. G. Ecology of bottom ice algae: I. Environmental controls and variability. J. Mar. Sys., 2(3):257-277, 1991.

Cota, G. F. Photoadaptation of high Arctic ice algae. Nature, 315:219-222, 1985.

Craig, H. and Hayward, T. Oxygen supersaturation in the ocean: Biological versus physical contributions. Science, 235:199-202, 1987. doi: 10.1126/science.235.4785.199.

Craig, H. Standard for reporting concentrations of deuterium and oxygen-18 in natural waters. Science, 133(3467):1833-1834, 1961a.

Craig, H. Isotopic variations in meteoric waters. Science, 133(3465):1702-1703, 1961b. doi: 10.1126/science.133.3465.1702.

Crusius, J. and Wanninkhof, R. Gas transfer velocities measured at low wind speed over a lake. Limnol. Oceanogr., 48(3):1010-1017, 2003.

DeVries, T., Deutsch, C., Primeau, F., Chang, B., and Devol, A. Global rates of watercolumn denitrification derived from nitrogen gas measurements. Nat. Geosci., 5(8):547$550,2012$.

Diaz, F. and Raimbault, P. Nitrogen regeneration and dissolved organic nitrogen release during spring in a NW Mediterranean coastal zone (Gulf of Lions): implications for the estimation of new production. Mar. Ecol. Prog. Ser., 197:51-65, 2000.

Dickson, A. G., Sabine, C. L., Christian, J. R., et al. Physical and thermodynamic data. In Guide to best practices for ocean $\mathrm{CO}_{2}$ measurements, PICES Special Publication 3, chapter 5. North Pacific Marine Science Organization, 2007.

Dore, J. E. and Karl, D. M. Nitrification in the euphotic zone as a source for nitrite, nitrate, and nitrous oxide at station aloha. Limnol. Oceanogr., 41(8):1619-1628, 1996.

D'souza, N. A., Kawarasaki, Y., Gantz, J. D., Lee, R. E., Beall, B. F. N., Shtarkman, Y. M., Kocer, Z. A., Rogers, S. O., Wildschutte, H., Bullerjahn, G. S., et al. Diatom assemblages promote ice formation in large lakes. ISME J., 7(8):1632-1640, 2013. 
Dubé, W. P., Brown, S. S., Osthoff, H. D., Nunley, M. R., Ciciora, S. J., Paris, M. W., McLaughlin, R. J., and Ravishankara, A. Aircraft instrument for simultaneous, in situ measurement of $\mathrm{NO}_{3}$ and $\mathrm{N}_{2} \mathrm{O}_{5}$ via pulsed cavity ring-down spectroscopy. Rev. Sci. Instrum., 77(3):034101, 2006.

Ducklow, H. W., Steinberg, D. K., and Buesseler, K. O. Upper ocean carbon export and the biological pump. Oceanography, 14(4):50-58, 2001.

Ducklow, H. W., Fraser, W. R., Meredith, M. P., Stammerjohn, S. E., Doney, S. C., Martinson, D. G., Sailley, S. F., Schofield, O. M., Steinberg, D. K., Venables, H. J., et al. West Antarctic Peninsula: an ice-dependent coastal marine ecosystem in transition. Oceanography, 26:190-203, 2013.

Dugdale, R. C. and Goering, J. J. Uptake of new and regenerated forms of nitrogen in primary productivity. Limnol. Oceanogr., 12(2):196-206, 1967.

Eisenstadt, D., Barkan, E., Luz, B., and Kaplan, A. Enrichment of oxygen heavy isotopes during photosynthesis in phytoplankton. Photosynth. Res., 103(2):97-103, 2010.

Else, B. G. T., Papakyriakou, T. N., Galley, R. J., Drennan, W. M., Miller, L. A., and Thomas, $\mathrm{H}$. Wintertime $\mathrm{CO}_{2}$ fluxes in an Arctic polynya using eddy covariance: Evidence for enhanced air-sea gas transfer during ice formation. J. Geophys. Res. Oceans, 116(C9), 2011. doi: 10.1029/2010JC006760. C00G03.

Emerson, S., Quay, P., Karl, D., Winn, C., Tupas, L., and Landry, M. Experimental determination of the organic carbon flux from open-ocean surface waters. Nature, 389 (6654):951-954, 1997.

Emerson, S. R. and Bushinsky, S. M. Oxygen concentrations and biological fluxes in the open ocean. Oceanography, 27(1):168-171, 2014. doi: 10.5670/oceanog.2014.20.

Emerson, S. Seasonal oxygen cycles and biological new production in surface waters of the subarctic Pacific Ocean. J. Geophys. Res. Oceans, 92(C6):6535-6544, 1987.

Emerson, S. Annual net community production and the biological carbon flux in the ocean. Global Biogeochem. Cy., 28(1):14-28, 2014.

Emerson, S. and Bushinsky, S. The role of bubbles during air-sea gas exchange. J. Geophys. Res. Oceans, 121(6):4360-4376, 2016. doi: 10.1002/2016JC011744.

Emerson, S., Quay, P., Stump, C., Wilbur, D., Knox, M., and Washington, S. O 2 , Ar, $\mathrm{N}_{2}$, and ${ }^{222} \mathrm{Rn}$ in the surface water of the subarctic ocean: Net biological $\mathrm{O}_{2}$ production. Global Biogeochem. Cy., 5(1):49-69, 1991.

Emerson, S., Quay, P., and Wheeler, P. A. Biological productivity determined from oxygen mass balance and incubation experiments. Deep-Sea Res. Pt. I, 40(11):2351-2358, 1993.

Emerson, S., Stump, C., and Nicholson, D. Net biological oxygen production in the ocean: Remote in situ measurements of $\mathrm{O}_{2}$ and $\mathrm{N}_{2}$ in surface waters. Global Biogeochem. Cy. 22:GB3023, 2008.

Emerson, S. R. and Stump, C. Net biological oxygen production in the ocean-II: Remote in situ measurements of $\mathrm{O}_{2}$ and $\mathrm{N}_{2}$ in subarctic Pacific surface waters. Deep Sea Res. I, 57(10):1255 - 1265, 2010. doi: 10.1016/j.dsr.2010.06.001. 
Eppley, R. W. Chlorophyll, photosynthesis and new production in the Southern California Bight. Prog. Oceanogr., 30(1-4):117-150, 1992.

Eppley, R. W. and Peterson, B. J. Particulate organic matter flux and planktonic new production in the deep ocean. Nature, 282:677-680, 1979.

Epstein, S. and Mayeda, T. Variation of $\mathrm{O}^{18}$ content of waters from natural sources. Geochim. Cosmochim. Acta, 4(5):213-224, 1953.

Estapa, M. L., Siegel, D. A., Buesseler, K. O., Stanley, R. H. R., Lomas, M. W., and Nelson, N. B. Decoupling of net community and export production on submesoscales in the sargasso sea. Global Biogeochem. Cy., 29(8):1266-1282, 2015.

Evans, W., Mathis, J. T., Cross, J. N., Bates, N. R., Frey, K. E., Else, B. G. T., Papkyriakou, T. N., DeGrandpre, M. D., Islam, F., Cai, W.-J., Chen, B., Yamamoto-Kawai, M., Carmack, E., Williams, W. J., and Takahashi, T. Sea-air $\mathrm{CO}_{2}$ exchange in the western Arctic coastal ocean. Global Biogeochem. Cy., 29(8):1190-1209, 2015. doi: 10.1002/2015GB005153.

Eveleth, R., Cassar, N., Sherrell, R., Ducklow, H., Meredith, M., Venables, H., Lin, Y., and $\mathrm{Li}, \mathrm{Z}$. Ice melt influence on summertime net community production along the Western Antarctic Peninsula. Deep-Sea Res. Pt. II, page In press, 2016.

Eveleth, R., Timmermans, M.-L., and Cassar, N. Physical and biological controls on oxygen saturation variability in the upper Arctic Ocean. J. Geophys. Res. Oceans, 119(11):7420$7432,2014$.

Fairall, C., Hare, J., Edson, J., and McGillis, W. Parameterization and micrometeorological measurement of air-sea gas transfer. Bound. Lay. Meteorol., 96(1-2):63-106, 2000.

Falkowski, P. G., Barber, R. T., and Smetacek, V. Biogeochemical controls and feedbacks on ocean primary production. Science, 281(5374):200-206, 1998.

Falkowski, P. G., Laws, E. A., Barber, R. T., and Murray, J. W. Phytoplankton and their role in primary, new, and export production. In Ocean Biogeochemistry, pages 99-121. Springer, 2003.

Fanning, K. A. and Torres, L. M. ${ }^{222} \mathrm{Rn}$ and ${ }^{226} \mathrm{Ra}$ : Indicators of sea-ice effects on air-sea gas exchange. Polar Research, 10(1):51-58, 1991.

Fassbender, A. J., Sabine, C. L., and Cronin, M. F. Net community production and calcification from 7 years of NOAA Station Papa Mooring measurements. Global Biogeochem. Cy., 30(2):250-267, 2016. doi: 10.1002/2015GB005205. 2015GB005205.

Feng, X., Faiia, A. M., and Posmentier, E. S. Seasonality of isotopes in precipitation: A global perspective. J. Geophys. Res. Pt. Atm., 114(D8), 2009. doi: 10.1029/2008JD011279. D08116.

Ferrell, R. T. and Himmelblau, D. M. Diffusion coefficients of nitrogen and oxygen in water. J. Chem. Eng. Data, 12(1):111-115, 1967.

Fine, R. A. Observations of CFCs and SF6 as ocean tracers. Ann. Rev. Mar. Sci., 3:173-195, 2011. 
Fitzwater, S. E., Johnson, K. S., Elrod, V. A., Ryan, J. P., Coletti, L. J., Tanner, S. J., Gordon, R. M., and Chavez, F. P. Iron, nutrient and phytoplankton biomass relationships in upwelled waters of the California coastal system. Cont. Shelf Res., 23(16):1523-1544, 2003.

Frankignoulle, M., Abril, G., Borges, A., Bourge, I., Canon, C., Delille, B., Libert, E., and Théate, J.-M. Carbon dioxide emission from European estuaries. Science, 282(5388): 434-436, 1998.

Frenette, J.-J., Thibeault, P., Lapierre, J.-F., and Hamilton, P. B. Presence of algae in freshwater ice cover of fluvial Lac Saint-Pierre (St. Lawrence River, Canada). J. Phycol., 44(2):284-291, 2008. doi: 10.1111/j.1529-8817.2008.00481.x.

Frew, N. The role of organic films in air-sea gas exchange. In Liss, P. and Duce, R., editors, The Sea Surface and Global Change, pages 121-171. Cambridge Univ. Press, New York, 1997.

Garcia, H. E. and Gordon, L. I. Oxygen solubility in seawater: Better fitting equations. Limnol. Oceanogr., 37(6):1307-1312, 1992.

Geen, G. H. and Hargrave, B. T. Primary and secondary production in Bras d'or Lake, Nova Scotia, Canada. Int. Ver. Theor. Angew. Limnol. Verh, 16:333-340, 1966.

Geen, G. H. Primary production in Bras d'Or Lake and other inland waters of Cape Breton Island, Nova Scotia. PhD thesis, Dalhousie University, 1965.

Giesbrecht, K. E., Hamme, R. C., and Emerson, S. R. Biological productivity along Line $\mathrm{P}$ in the subarctic northeast Pacific: In situ versus incubation-based methods. Global Biogeochem. Cy., 26(3), 2012. doi: 10.1029/2012GB004349. GB3028.

Glibert, P. and Goldman, J. Rapid ammonium uptake by marine phytoplankton. Mar. Biol. Lett, 2:25-31, 1981.

Golden, K. M., Eicken, H., Heaton, A. L., Miner, J., Pringle, D. J., and Zhu, J. Thermal evolution of permeability and microstructure in sea ice. Geophys. Res. Lett., 34(16), 2007. doi: 10.1029/2007GL030447. L16501.

Goldman, J. C., Taylor, C. D., and Glibert, P. M. Nonlinear time-course uptake of carbon and ammonium by marine phytoplankton. Mar. Ecol. Prog. Ser, 6:137-148, 1981.

Goldman, J. A. L., Kranz, S. A., Young, J. N., Tortell, P. D., Stanley, R. H. R., Bender, M. L., and Morel, F. M. M. Gross and net production during the spring bloom along the Western Antarctic Peninsula. New Phytol., 205(1):182-191, 2015.

Gonzalez-Valencia, R., Magana-Rodriguez, F., Gerardo-Nieto, O., Sepulveda-Jauregui, A., Martinez-Cruz, K., Walter Anthony, K., Baer, D., and Thalasso, F. In situ measurement of dissolved methane and carbon dioxide in freshwater ecosystems by off-axis integrated cavity output spectroscopy. Environ. Sci. Technol., 48(19):11421-11428, 2014.

Gosink, T. A., Pearson, J. G., and Kelley, J. J. Gas movement through sea ice. Nature, 263 (5572):41-42, 1976. 
Gosselin, M., Legendre, L., Therriault, J.-C., Demers, S., and Rochet, M. Physical control of the horizontal patchiness of sea-ice microalgae. Mar. Ecol. Prog. Ser., 29(3):289-298, 1986.

Graham, W. M. and Largier, J. L. Upwelling shadows as nearshore retention sites: the example of northern Monterey Bay. Cont. Shelf Res., 17(5):509-532, 1997.

Greenan, B. J. W., Petrie, B. D., Harrison, W. G., and Oakey, N. S. Are the spring and fall blooms on the Scotian Shelf related to short-term physical events? Cont. Shelf Res., 24 (4):603-625, 2004.

Grefe, I. and Kaiser, J. Equilibrator-based measurements of dissolved nitrous oxide in the surface ocean using an integrated cavity output laser absorption spectrometer. Ocean Sci., 10(3):501-512, 2014.

Gregg, M. C. Diapycnal mixing in the thermocline: A review. J. Geophys. Res. Oceans, 92 (C5):5249-5286, 1987. doi: 10.1029/JC092iC05p05249.

Grundle, D. S., Juniper, S. K., and Giesbrecht, K. E. Euphotic zone nitrification in the NE subarctic Pacific: Implications for measurements of new production. Mar. Chem., 155: 113-123, 2013.

Guillemette, F. and del Giorgio, P. A. Reconstructing the various facets of dissolved organic carbon bioavailability in freshwater ecosystems. Limnol. Oceanogr., 56(2):734-748, 2011.

Gurbutt, P. A. and Petrie, B. Circulation in the Bras d'Or Lakes. Est. Coast. Shelf Sci., 41 (6):611-630, 1995.

Halsey, K. H., Milligan, A. J., and Behrenfeld, M. J. Physiological optimization underlies growth rate-independent chlorophyll-specific gross and net primary production. Photosynth. Res., 103(2):125-137, 2010.

Halsey, K. H., O’Malley, R. T., Graff, J. R., Milligan, A. J., and Behrenfeld, M. J. A common partitioning strategy for photosynthetic products in evolutionarily distinct phytoplankton species. New Phytol., 198(4):1030-1038, 2013.

Hamme, R. C. and Emerson, S. R. Deep-sea nutrient loss inferred from the dissolved $\mathrm{N}_{2} / \mathrm{Ar}$ ratio. Geophys. Res. Lett., 40:1149-1153, 2013. doi: 10.1002/grl.50275.

Hamme, R. and Emerson, S. The solubility of neon, nitrogen and argon in distilled water and seawater. Deep-Sea Res. Pt. I, 51(11):1517-1528, 2004a.

Hamme, R. C. and Emerson, S. R. Mechanisms controlling the global oceanic distribution of the inert gases argon, nitrogen and neon. Geophys. Res. Lett., 29(23):35-1-35-4, 2002. doi: $10.1029 / 2002$ GL015273.

Hamme, R. C. and Emerson, S. R. Measurement of dissolved neon by isotope dilution using a quadrupole mass spectrometer. Mar. Chem., 91(1-4):53-64, 2004b. doi: 10.1016/j. marchem.2004.05.001.

Hamme, R. C. and Emerson, S. R. Constraining bubble dynamics and mixing with dissolved gases: Implications for productivity measurements by oxygen mass balance. J. Mar. Res., 64:73-95, 2006. doi: 10.1357/002224006776412322. 
Hamme, R. C. and Severinghaus, J. P. Trace gas disequilibria during deep-water formation. Deep Sea Res. Pt. I, 54(6):939-950, 2007. doi: 10.1016/j.dsr.2007.03.008.

Hamme, R. C., Cassar, N., Lance, V. P., Vaillancourt, R. D., Bender, M. L., Strutton, P. G., Moore, T. S., DeGrandpre, M. D., Sabine, C. L., Ho, D. T., and Hargreaves, B. R. Dissolved $\mathrm{O}_{2} / \mathrm{Ar}$ and other methods reveal rapid changes in productivity during a Lagrangian experiment in the Southern Ocean. J. Geophys. Res. Oceans, 117(C4): C00F12, 2012. doi: 10.1029/2011JC007046.

Hampton, S. E., Moore, M. V., Ozersky, T., Stanley, E. H., Polashenski, C. M., and Galloway, A. W. E. Heating up a cold subject: prospects for under-ice plankton research in lakes. J. Plankton. Res., 37(2):277-284, 2015.

Hansell, D. A. and Carlson, C. A. Net community production of dissolved organic carbon. Global Biogeochem. Cy., 12(3):443-453, 1998.

Hargrave, B. T. and Geen, G. H. Effects of copepod grazing on two natural phytoplankton populations. J. Fish. Board Can., 27(8):1395-1403, 1970.

Haskell, W. Z., Prokopenko, M. G., Stanley, R. H. R., and Knapp, A. N. Estimates of vertical turbulent mixing used to determine a vertical gradient in net and gross oxygen production in the oligotrophic South Pacific Gyre. Geophys. Res. Lett., 2016a. doi: 10.1002/2016GL069523. 2016GL069523.

Haskell, W. Z., Prokopenko, M. G., Hammond, D. E., Stanley, R. H. R., Berelson, W. M., Baronas, J. J., Fleming, J. C., and Aluwihare, L. An organic carbon budget for coastal Southern California determined by estimates of vertical nutrient flux, net community production and export. Deep-Sea Res. Pt. I, 116:49-76, 2016b.

Helman, Y., Barkan, E., Eisenstadt, D., Luz, B., and Kaplan, A. Fractionation of the three stable oxygen isotopes by oxygen-producing and oxygen-consuming reactions in photosynthetic organisms. Plant Physiol., 138(4):2292-2298, 2005.

Hendricks, M. B., Bender, M. L., and Barnett, B. A. Net and gross $\mathrm{O}_{2}$ production in the Southern Ocean from measurements of biological $\mathrm{O}_{2}$ saturation and its triple isotope composition. Deep-Sea Res. Pt. I, 51(11):1541-1561, 2004.

Hendricks, M. B., Bender, M. L., Barnett, B. A., Strutton, P., and Chavez, F. P. Triple oxygen isotope composition of dissolved $\mathrm{O}_{2}$ in the equatorial Pacific: A tracer of mixing, production, and respiration. J. Geophys. Res. Oceans, 110(C12), 2005. doi: 10.1029/ 2004JC002735. C12021.

Henson, S. A., Sanders, R., Madsen, E., Morris, P. J., Le Moigne, F., and Quartly, G. D. A reduced estimate of the strength of the ocean's biological carbon pump. Geophys. Res. Lett., 38(4), 2011. doi: 10.1029/2011GL046735. L04606.

Ho, D. T., Law, C. S., Smith, M. J., Schlosser, P., Harvey, M., and Hill, P. Measurements of air-sea gas exchange at high wind speeds in the Southern Ocean: Implications for global parameterizations. Geophys. Res. Lett., 33(16), 2006. doi: 10.1029/2006GL026817. L16611. 
Ho, D. T., Sabine, C. L., Hebert, D., Ullman, D. S., Wanninkhof, R., Hamme, R. C., Strutton, P. G., Hales, B., Edson, J. B., and Hargreaves, B. R. Southern ocean gas exchange experiment: setting the stage. J. Geophys. Res. Oceans, 116(C4), 2011a. doi: 10.1029/2010JC006852. C00F08.

Ho, D. T., Schlosser, P., and Orton, P. M. On factors controlling air-water gas exchange in a large tidal river. Estuar. Coast, 34(6):1103-1116, 2011 b.

Holmes, R. M., Aminot, A., Kérouel, R., Hooker, B. A., and Peterson, B. J. A simple and precise method for measuring ammonium in marine and freshwater ecosystems. Can. J. Fish. Aquat. Sci., 56(10):1801-1808, 1999.

Hood, E. M., Howes, B. L., and Jenkins, W. J. Dissolved gas dynamics in perennially ice-covered Lake Fryxell, Antarctica. Limnol. Oceanogr., 43(2):265-272, 1998.

Hsu, S. A., Meindl, E. A., and Gilhousen, D. B. Determining the power-law wind-profile exponent under near-neutral stability conditions at sea. J. Appl. Meteor., 33(6):757-765, 1994.

Hutchins, D. and Bruland, K. Fe, Zn, Mn and N transfer between size classes in a coastal phytoplankton community: Trace metal and major nutrient recycling compared. J. Mar. Res., 53(2):297-313, 1995.

IAEA/WMO. Global Network of Isotopes in Precipitation. The GNIP Database. http: //www . iaea.org/water, 2016.

Idso, S. B. and Gilbert, R. G. On the universality of the Poole and Atkins Secchi disk-light extinction equation. J. Appl. Ecol., 11(1):399-401, 1974.

Ito, T. and Deutsch, C. Understanding the saturation state of argon in the thermocline: The role of air-sea gas exchange and diapycnal mixing. Global Biogeochem. Cy., 20(3), 2006. doi: 10.1029/2005GB002655. GB3019.

Ito, T., Deutsch, C., Emerson, S., and Hamme, R. C. Impact of diapycnal mixing on the saturation state of argon in the subtropical North Pacific. Geophys. Res. Lett., 34:L09602, 2007. doi: 10.1029/2006GL029209.

Ito, T., Hamme, R. C., and Emerson, S. Temporal and spatial variability of noble gas tracers in the North Pacific. J. Geophys. Res. Oceans, 116(C8), 2011. doi: 10.1029/2010JC006828. C08039.

Jähne, B., Huber, W., Dutzi, A., Wais, T., and Ilmberger, J. Wind/wave-tunnel experiment on the Schmidt number and wave field dependence of air/water gas exchange. In Gas transfer at water surfaces, pages 303-309. Springer, 1984.

Jähne, B., Heinz, G., and Dietrich, W. Measurement of the diffusion coefficients of sparingly soluble gases in water. J. Geophys. Res. Oceans, 92(C10):10767-10776, 1987a. doi: 10. 1029/JC092iC10p10767.

Jähne, B., Münnich, K. O., Bösinger, R., Dutzi, A., Huber, W., and Libner, P. On the parameters influencing air-water gas exchange. J. Geophys. Res. Oceans, 92(C2):19371949, 1987b. 
Jasechko, S., Sharp, Z. D., Gibson, J. J., Birks, S. J., Yi, Y., and Fawcett, P. J. Terrestrial water fluxes dominated by transpiration. Nature, 496(7445):347-350, 2013.

Jenkins, W. J., Lott, D. E., Cahill, K., Curtice, J., and Landry, P. Sampling and measuring helium isotopes and tritium in seawater. Technical Report 14, IOCCP report, 2010.

Jenkins, W. J. Studying subtropical thermocline ventilation and circulation using tritium and ${ }^{3}$ He. J. Geophys. Res. Oceans, 103(C8):15817-15831, 1998. doi: 10.1029/98JC00141.

Johnson, K. S. Simultaneous measurements of nitrate, oxygen, and carbon dioxide on oceanographic moorings: Observing the Redfield ratio in real time. Limnol. Oceanogr., $55(2): 615,2010$.

Johnson, K. S., Plant, J. N., Riser, S. C., and Gilbert, D. Air oxygen calibration of oxygen optodes on a profiling float array. J. Atmos. Ocean. Tech., 32(11):2160-2172, 2015.

Jonsson, B. F., Doney, S. C., Dunne, J., and Bender, M. Evaluation of the Southern Ocean $\mathrm{O}_{2} /$ Ar-based NCP estimates in a model framework. J. Geophys. Res. Pt. Biogeosci., 118 (2):385-399, 2013.

JPL/OBPG/RSMAS. GHRSST Level 2P Global Skin Sea Surface Temperature from the Moderate Resolution Imaging Spectroradiometer (MODIS) on the NASA Aqua satellite. Ver. 1.0. PO.DAAC, CA, USA. Dataset accessed [2016-06-21]., 2006a.

JPL/OBPG/RSMAS. GHRSST Level 2P Global Skin Sea Surface Temperature from the Moderate Resolution Imaging Spectroradiometer (MODIS) on the NASA Terra satellite. Ver. 1.0.Dataset accessed [2016-06-21], 2006b.

Juranek, L. W. and Quay, P. D. In vitro and in situ gross primary and net community production in the North Pacific Subtropical Gyre using labeled and natural abundance isotopes of dissolved $\mathrm{O}_{2}$. Global Biogeochem. Cy., 19(3), 2005. doi: 10.1029/2004GB002384. GB3009.

Juranek, L. W. and Quay, P. D. Using triple isotopes of dissolved oxygen to evaluate global marine productivity. Ann. Rev. Mar. Sci., 5:503-524, 2013.

Juranek, L. W., Hamme, R. C., Kaiser, J., Wanninkhof, R., and Quay, P. D. Evidence of $\mathrm{O}_{2}$ consumption in underway seawater lines: Implications for air-sea $\mathrm{O}_{2}$ and $\mathrm{CO}_{2}$ fluxes. Geophys. Res. Lett., 37(1), 2010.

Kaiser, J. Technical note: Consistent calculation of aquatic gross production from oxygen triple isotope measurements. Biogeosciences, 8(7):1793-1811, 2011.

Kameyama, S., Tanimoto, H., Inomata, S., Tsunogai, U., Ooki, A., Yokouchi, Y., Takeda, S., Obata, H., and Uematsu, M. Equilibrator inlet-proton transfer reaction-mass spectrometry (EI-PTR-MS) for sensitive, high-resolution measurement of dimethyl sulfide dissolved in seawater. Anal. Chem., 81(21):9021-9026, 2009.

Kana, T. M., Darkangelo, C., Hunt, M. D., Oldham, J. B., Bennett, G. E., and Cornwell, J. C. Membrane inlet mass spectrometer for rapid high-precision determination of $\mathrm{N}_{2}$, $\mathrm{O}_{2}$, and Ar in environmental water samples. Anal. Chem., 66(23):4166-4170, 1994. 
Kanda, J., Laws, E., Saino, T., and Hattori, A. An evaluation of isotope dilution effect from conventional data sets of ${ }^{15} \mathrm{~N}$ uptake experiments. J. Plankton Res., 9(1):79-90, 1987.

Karl, D. M., Christian, J. R., Dore, J. E., Hebel, D. V., Letelier, R. M., Tupas, L. M., and Winn, C. D. Seasonal and interannual variability in primary production and particle flux at Station ALOHA. Deep-Sea Res. Pt. II, 43(2):539-568, 1996.

Karl, D. M., Hebel, D. V., Björkman, K., and Letelier, R. M. The role of dissolved organic matter release in the productivity of the oligotrophic North Pacific Ocean. Limnol. Oceanogr., 43:1270-1286, 1998.

Karl, D. M., Laws, E. A., Morris, P., Emerson, S., et al. Metabolic balance of the open sea. Nature, 426(6962):32-32, 2003.

Khatiwala, S., Primeau, F., and Hall, T. Reconstruction of the history of anthropogenic $\mathrm{CO}_{2}$ concentrations in the ocean. Nature, 462(7271):346-349, 2009.

King, D. and Saltzman, E. Measurement of the diffusion coefficient of sulfur hexafluoride in water. J. Geophys. Res. Oceans, 100(C4):7083-7088, 1995.

Knauer, G. A., Martin, J. H., and Bruland, K. W. Fluxes of particulate carbon, nitrogen, and phosphorus in the upper water column of the northeast Pacific. Deep-Sea Res. Pt. A, 26(1):97-108, 1979.

Kohout, A. L. and Meylan, M. H. An elastic plate model for wave attenuation and ice floe breaking in the marginal ice zone. J. Geophys. Res. Oceans, 113(C9), 2008.

Krall, K. E. and Jähne, B. First laboratory study of air-sea gas exchange at hurricane wind speeds. Ocean Sci., 10(2):257-265, 2014.

Kudela, R. M. and Dugdale, R. C. Nutrient regulation of phytoplankton productivity in Monterey Bay, California. Deep-Sea Res. Pt. II, 47(5):1023-1053, 2000.

Kudela, R. M. Characterization and prediction of planktonic nitrogenous nutrition and new production in Monterey Bay, California: nutrition and physiological interactions. $\mathrm{PhD}$ thesis, University of Southern California, 1995.

Kunze, E., Dower, J. F., Beveridge, I., Dewey, R., and Bartlett, K. P. Observations of biologically generated turbulence in a coastal inlet. Science, 313(5794):1768-1770, 2006.

Landais, A., Barkan, E., and Luz, B. Record of $\delta^{18} \mathrm{O}$ and ${ }^{17} \mathrm{O}$-excess in ice from Vostok Antarctica during the last 150,000 years. Geophys. Res. Lett., 35(2), 2008.

Large, W. G. and Pond, S. Open ocean momentum flux measurements in moderate to strong winds. J. Phys. Oceanogr., 11(3):324-336, 1981.

Large, W. G., McWilliams, J. C., and Doney, S. C. Oceanic vertical mixing: A review and a model with a nonlocal boundary layer parameterization. Rev. Geophys., 32(4):363-403, 1994.

Laws, E. A. Photosynthetic quotients, new production and net community production in the open ocean. Deep-Sea Res. Pt. A, 38(1):143-167, 1991. 
Laws, E. A., Falkowski, P. G., Smith, W. O., Ducklow, H., and McCarthy, J. J. Temperature effects on export production in the open ocean. Global Biogeochem. Cy., 14(4):1231-1246, 2000 .

Le Quéré, C., Moriarty, R., Andrew, R. M., Canadell, J. G., Sitch, S., Korsbakken, J. I., Friedlingstein, P., Peters, G. P., Andres, R. J., Boden, T., et al. Global carbon budget 2015. Earth Syst. Sci. Data, 7(2):349-396, 2015.

Ledwell, J. R., Watson, A. J., and Law, C. S. Evidence for slow mixing across the pycnocline from an open-ocean tracer-release experiment. Nature, 364(6439):701-703, 1993.

Ledwell, J., Montgomery, E., Polzin, K., Laurent, L. S., Schmitt, R., and Toole, J. Evidence for enhanced mixing over rough topography in the abyssal ocean. Nature, 403(6766): 179-182, 2000.

Legendre, L., Ingram, R. G., and Poulin, M. Physical control of phytoplankton production under sea ice (Manitounuk Sound, Hudson Bay). Can. J. Fish. Aquat. Sci., 38(11):1385$1392,1981$.

Legge, O. J., Bakker, D. C., Johnson, M. T., Meredith, M. P., Venables, H. J., Brown, P. J., and Lee, G. A. The seasonal cycle of ocean-atmosphere $\mathrm{CO}_{2}$ flux in Ryder Bay, west Antarctic Peninsula. Geophys. Res. Lett., 42(8):2934-2942, 2015.

LeGrande, A. N. and Schmidt, G. A. Global gridded data set of the oxygen isotopic composition in seawater. Geophys. Res. Lett., 33(12), 2006. doi: 10.1029/2006GL026011. L12604.

Li, S., Levin, N. E., and Chesson, L. A. Continental scale variation in ${ }^{17} \mathrm{O}$-excess of meteoric waters in the United States. Geochim. Cosmochim. Acta, 164:110-126, 2015.

Liang, J.-H., Deutsch, C., McWilliams, J. C., Baschek, B., Sullivan, P. P., and Chiba, D. Parameterizing bubble-mediated air-sea gas exchange and its effect on ocean ventilation. Global Biogeochem. Cy., 27(3):894-905, 2013.

Light, B., Grenfell, T. C., and Perovich, D. K. Transmission and absorption of solar radiation by Arctic sea ice during the melt season. J. Geophys. Res. Oceans, 113(C3), 2008. doi: 10.1029/2006JC003977. C03023.

Light, B., Perovich, D. K., Webster, M. A., Polashenski, C., and Dadic, R. Optical properties of melting first-year Arctic sea ice. J. Geophys. Res. Oceans, 120(11):7657-7675, 2015. doi: $10.1002 / 2015 J C 011163$.

Liss, P. S. and Merlivat, L. Air-sea gas exchange rates: Introduction and synthesis. In The role of air-sea exchange in geochemical cycling, pages 113-127. Springer, 1986.

Livingstone, D. M. and Imboden, D. M. The non-linear influence of wind-speed variability on gas transfer in lakes. Tellus B, 45(3):275-295, 1993.

Lohrenz, S. E., Knauer, G. A., Asper, V. L., Tuel, M., Michaels, A. F., and Knap, A. H. Seasonal variability in primary production and particle flux in the northwestern sargasso sea: Us jgofs bermuda atlantic time-series study. Deep-Sea Res. Pt. A, 39(7):1373-1391, 1992. 
Longhurst, A. R. and Harrison, W. G. The biological pump: profiles of plankton production and consumption in the upper ocean. Prog. Oceanogr., 22(1):47-123, 1989.

Loose, B., McGillis, W. R., Schlosser, P., Perovich, D., and Takahashi, T. Effects of freezing, growth, and ice cover on gas transport processes in laboratory seawater experiments. Geophys. Res. Lett., 36(5), 2009a. doi: 10.1029/2008GL036318. L05603.

Loose, B., McGillis, W., Schlosser, P., Perovich, D., and Takahashi, T. Effects of freezing, growth, and ice cover on gas transport processes in laboratory seawater experiments. Geophys. Res. Lett., 36(5), 2009b.

Loose, B., Schlosser, P., Perovich, D., Ringelberg, D., Ho, D. T., Takahashi, T., RichterMenge, J., Reynolds, C. M., McGillis, W., and Tison, J.-L. Gas diffusion through columnar laboratory sea ice: Implications for mixed-layer ventilation of $\mathrm{CO}_{2}$ in the seasonal ice zone. Tellus B, 63(1):23-39, 2011a.

Loose, B., McGillis, W. R., Perovich, D., Zappa, C. J., and Schlosser, P. A parameter model of gas exchange for the seasonal sea ice zone. Ocean Sci., 10(1):17-28, 2014. doi: 10.5194/os-10-17-2014.

Loose, B. and Jenkins, W. J. The five stable noble gases are sensitive unambiguous tracers of glacial meltwater. Geophys. Res. Lett., 41(8):2835-2841, 2014.

Loose, B. and Schlosser, P. Sea ice and its effect on $\mathrm{CO}_{2}$ flux between the atmosphere and the Southern Ocean interior. J. Geophys. Res. Oceans, 116(C11), 2011.

Loose, B., Miller, L. A., Elliott, S., and Papakyriakou, T. Sea ice biogeochemistry and material transport across the frozen interface. Oceanography, 24:202-218, 2011b.

Loose, B., Jenkins, W. J., Moriarty, R., Brown, P., Jullion, L., Naveira Garabato, A. C., Torres Valdes, S., Hoppema, M., Ballentine, C., and Meredith, M. P. Estimating the recharge properties of the deep ocean using noble gases and helium isotopes. J. Geophys. Res. Oceans, 2016. doi: 10.1002/2016JC011809.

Lovely, A., Loose, B., Schlosser, P., McGillis, W., Zappa, C., Perovich, D., Brown, S., Morell, T., Hsueh, D., and Friedrich, R. The Gas Transfer through Polar Sea ice experiment: Insights into the rates and pathways that determine geochemical fluxes. J. Geophys. Res. Oceans, 120(12):8177-8194, 2015. doi: 10.1002/2014JC010607.

Ludin, A., Weppernig, G., Bönisch, G., and Schlosser, P. Mass spectrometric measurement of helium isotopes and tritium in water samples. Technical report, Lamont-Doherty Earth Observatory, Palisades, NY, 1998.

Luz, B. and Barkan, E. Assessment of oceanic productivity with the triple-isotope composition of dissolved oxygen. Science, 288:2028-2031, 2000.

Luz, B. and Barkan, E. The isotopic ratios ${ }^{17} \mathrm{O} /{ }^{16} \mathrm{O}$ and ${ }^{18} \mathrm{O} /{ }^{16} \mathrm{O}$ in molecular oxygen and their significance in biogeochemistry. Geochim. Cosmochim. Acta, 69(5):1099-1110, 2005.

Luz, B. and Barkan, E. Net and gross oxygen production from $\mathrm{O}_{2} / \mathrm{Ar},{ }^{17} \mathrm{O} /{ }^{16} \mathrm{O}$ and ${ }^{18} \mathrm{O} /{ }^{16} \mathrm{O}$ ratios. Aquat. Microb. Ecol, 56:133-145, 2009. 
Luz, B. and Barkan, E. Variations of ${ }^{17} \mathrm{O} /{ }^{16} \mathrm{O}$ and ${ }^{18} \mathrm{O} /{ }^{16} \mathrm{O}$ in meteoric waters. Geochim. Cosmochim. Acta, 74(22):6276-6286, 2010.

Luz, B. and Barkan, E. Proper estimation of marine gross $\mathrm{O}_{2}$ production with ${ }^{17} \mathrm{O} /{ }^{16} \mathrm{O}$ and ${ }^{18} \mathrm{O} /{ }^{16} \mathrm{O}$ ratios of dissolved $\mathrm{O}_{2}$. Geophys. Res. Lett., 38(19), 2011.

Lv, Y., Yu, X., Tu, S.-T., Yan, J., and Dahlquist, E. Wetting of polypropylene hollow fiber membrane contactors. J. Membr. Sci., 362(1):444-452, 2010.

Macdonald, R. W., Carmack, E. C., and Paton, D. W. Using the $\delta^{18} \mathrm{O}$ composition in landfast ice as a record of Arctic estuarine processes. Mar. Chem., 65(1):3-24, 1999.

Macdonald, R. W., Paton, D. W., Carmack, E. C., and Omstedt, A. The freshwater budget and under-ice spreading of Mackenzie River water in the Canadian Beaufort Sea based on salinity and ${ }^{18} \mathrm{O} /{ }^{16} \mathrm{O}$ measurements in water and ice. J. Geophys. Res. Oceans, 100(C1): 895-919, 1995. doi: 10.1029/94JC02700.

MacGilchrist, G. A., Garabato, A. C. N., Tsubouchi, T., Bacon, S., Torres-Valdés, S., and Azetsu-Scott, K. The Arctic Ocean carbon sink. Deep-Sea Res. Pt. I, 86:39-55, 2014.

Machler, L., Brennwald, M. S., and Kipfer, R. Membrane inlet mass spectrometer for the quasi-continuous on-site analysis of dissolved gases in groundwater. Environ. Sci. Technol., 46(15):8288-8296, August 2012.

MacIsaac, J. J., Dugdale, R. C., Barber, R. T., Blasco, D., and Packard, T. T. Primary production cycle in an upwelling center. Deep-Sea Res. Pt. A, 32(5):503-529, 1985.

MacMahon, D. Half-life evaluations for ${ }^{3} \mathrm{H},{ }^{90} \mathrm{Sr}$, and ${ }^{90} \mathrm{Y}$. Appl. Radiat. Isotopes, 64(10): 1417-1419, 2006.

Mamyrin, B., Anufriyev, G., Kamenskiy, I., and Tolstikhin, I. Determination of the isotopic composition of atmospheric helium. Geochem. Int., 7:498-505, 1970.

Manning, C. C. and Howard, E. M. calcGOP: Matlab functions for the calculation of gross oxygen production from triple oxygen isotope data. http://github.com/caramanning/ calcGOP, 2016.

Manning, C. C. and Nicholson, D. P. gas_toolbox: Updated release for Manning et al. GTWS-7 proceedings. doi: 10.5281/zenodo.46569, 2016.

Manning, C. C., Stanley, R. H. R., and Lott, D. E. Continuous measurements of dissolved $\mathrm{Ne}, \mathrm{Ar}, \mathrm{Kr}$, and Xe with a field-deployable gas equilibration mass spectrometer. Anal. Chem., 88(6):3040-3048, 2016a. doi: 10.1021/acs.analchem.5b03102.

Manning, C. C., Stanley, R. H. R., Nicholson, D. P., Smith, J. M., Pennington, J. T., Fewings, M., Squibb, M. E., and Chavez, F. P. Quantifying air-sea gas exchange using noble gases in a coastal upwelling zone. J. Geophys. Res. Oceans, 2016b. Manuscript 2016JC012306, in revision.

Manning, C. C., Stanley, R. H. R., Nicholson, D. P., and Squibb, M. E. Quantifying air-sea gas exchange using noble gases in a coastal upwelling zone. IOP Conf. Ser.: Earth Envir. Sci., 88(6):3040-3048, 2016c. doi: 10.1088/1755-1315/35/1/012017. 
Marandino, C., De Bruyn, W. J., Miller, S. D., and Saltzman, E. S. Open ocean DMS air/sea fluxes over the eastern South Pacific Ocean. Atmos. Chem. Phys., 9:345-356, 2009.

Marra, J. Approaches to the measurement of plankton production. In Phytoplankton Productivity: Carbon assimilation in marine and freshwater ecosystems, pages 78-108. Blackwell Publishing, Ltd. Cambridge, Blackwells, 2002.

Marra, J. Net and gross productivity: weighing in with ${ }^{14}$ C. Aquat. Microb. Ecol., 56: 123-131, 2009.

Martin, J., Coale, K., Johnson, K., Fitzwater, S., Gordon, R., Tanner, S., Hunter, C., Elrod, V., Nowicki, J., Coley, T., et al. Testing the iron hypothesis in ecosystems of the equatorial Pacific Ocean. Nature, 371(6493):123-129, 1994.

Maslanik, J., Stroeve, J., Fowler, C., and Emery, W. Distribution and trends in Arctic sea ice age through spring 2011. Geophys. Res. Lett., 38(13), 2011.

McCarthy, J. J. The uptake of urea by natural populations of marine phytoplankton. Limnol. Oceanogr., 17(5):738-748, 1972. doi: 10.4319/lo.1972.17.5.0738.

McDougall, T. J. and Barker, P. M. Getting started with TEOS-10 and the Gibbs Seawater (GSW) Oceanographic Toolbox. SCOR/IAPSO WG127, 2011. ISBN 978-0-646-55621-5.

McNeil, C., Katz, D., Wanninkhof, R., and Johnson, B. Continuous shipboard sampling of gas tension, oxygen and nitrogen. Deep-Sea Res. Pt. I, 52(9):1767-1785, 2005.

McNeil, C., D'Asaro, E., Johnson, B., and Horn, M. A gas tension device with response times of minutes. J. Atmos. Ocean. Tech., 23(11):1539-1558, 2006.

McPhee, M. G. Turbulent heat flux in the upper ocean under sea ice. J. Geophys. Res. Oceans, 97(C4):5365-5379, 1992. doi: 10.1029/92JC00239.

Meijer, H. A. J. and Li, W. J. The use of electrolysis for accurate $\delta^{17} \mathrm{O}$ and $\delta^{18} \mathrm{O}$ isotope measurements in water. Isot. Environ Healt. Sci., 34(4):349-369, 1998.

Mesarchaki, E., Kräuter, C., Krall, K. E., Bopp, M., Helleis, F., Williams, J., and Jähne, B. Measuring air-sea gas exchange velocities in a large scale annular wind-wave tank. Ocean Sci., 11:121-138, 2015a.

Mesarchaki, E., Kräuter, C., Krall, K., Bopp, M., Helleis, F., Williams, J., and Jähne, B. Measuring air-sea gas-exchange velocities in a large-scale annular wind-wave tank. Ocean Sci., 11(1):121-138, 2015b.

Michaels, A. F., Silver, M. W., Gowing, M. M., and Knauer, G. A. Cryptic zooplankton "swimmers" in upper ocean sediment traps. Deep-Sea Res. Pt. A, 37(8):1285-1296, 1990.

Michel, C., Nielsen, T. G., Nozais, C., and Gosselin, M. Significance of sedimentation and grazing by ice micro-and meiofauna for carbon cycling in annual sea ice (northern Baffin Bay). Aquat. Microb. Ecol., 30(1):57-68, 2002.

Moore, C., Mills, M., Arrigo, K., Berman-Frank, I., Bopp, L., Boyd, P., Galbraith, E., Geider, R. J., Guieu, C., Jaccard, S., et al. Processes and patterns of oceanic nutrient limitation. Nature Geoscience, 6(9):701-710, 2013. 
Moreau, S., Vancoppenolle, M., Zhou, J., Tison, J.-L., Delille, B., and Goosse, H. Modelling argon dynamics in first-year sea ice. Ocean Model., 73:1-18, 2014.

Morel, A. and Berthon, J.-F. Surface pigments, algal biomass profiles, and potential production of the euphotic layer: Relationships reinvestigated in view of remote-sensing applications. Limnol. Oceanogr., 34(8):1545-1562, 1989.

Morison, J. H., McPhee, M. G., Curtin, T. B., and Paulson, C. A. The oceanography of winter leads. J. Geophys. Res, 97(11):199-11, 1992.

Mucci, A. and Page, P. The water chemistry of cruise 85-036 on Bras d'Or Lake, Cape Breton Island, Nova Scotia. Current Research, Part A, Geological Survey of Canada, Paper 87-1A:17-24, 1987.

Mundy, C., Gosselin, M., Ehn, J., Gratton, Y., Rossnagel, A., Barber, D. G., Martin, J., Tremblay, J.-É., Palmer, M., Arrigo, K. R., et al. Contribution of under-ice primary production to an ice-edge upwelling phytoplankton bloom in the Canadian Beaufort Sea. Geophys. Res. Lett., 36(17), 2009.

Munro, D. R., Quay, P. D., Juranek, L. W., and Goericke, R. Biological production rates off the Southern California coast estimated from triple $\mathrm{O}_{2}$ isotopes and $\mathrm{O}_{2}$ :Ar gas ratios. Limnol. Oceanogr., 58(4):1312-1328, 2013.

Naegler, T., Ciais, P., Rodgers, K., and Levin, I. Excess radiocarbon constraints on air-sea gas exchange and the uptake of $\mathrm{CO}_{2}$ by the oceans. Geophys. Res. Lett., 33(11), 2006.

Nagai, T., Gruber, N., Frenzel, H., Lachkar, Z., McWilliams, J., and Plattner, G.-K. Dominant role of eddies and filaments in the offshore transport of carbon and nutrients in the California Current System. J. Geophys. Res. Oceans, 120:5318-5341, 2015.

Nagel, L., Krall, K., and Jähne, B. Comparative heat and gas exchange measurements in the Heidelberg Aeolotron, a large annular wind-wave tank. Ocean Sci., 11:111-120, 2015.

Nicholls, R. J. and Cazenave, A. Sea-level rise and its impact on coastal zones. Science, 328 (5985):1517-1520, 2010.

Nicholson, D. P., Khatiwala, S., and Heimbach, P. Noble gas tracers of ventilation during deep-water formation in the Weddell Sea. IOP Conf. Ser. Earth Environ. Sci, 35(1): 012019, 2016. doi: 10.1088/1755-1315/35/1/012019.

Nicholson, D., Emerson, S., and Eriksen, C. C. Net community production in the deep euphotic zone of the subtropical North Pacific gyre from glider surveys. Limnol. Oeanogr., 53(5, part 2):2226-2236, 2008. doi: 10.4319/lo.2008.53.5_part_2.2226.

Nicholson, D., Emerson, S., Caillon, N., Jouzel, J., and Hamme, R. C. Constraining ventilation during deepwater formation using deep ocean measurements of the dissolved gas ratios ${ }^{40} \mathrm{Ar} /{ }^{36} \mathrm{Ar}, \mathrm{N}_{2} / \mathrm{Ar}$, and $\mathrm{Kr} / \mathrm{Ar}$. J. Geophys. Res. Oceans, 115(C11), 2010.

Nicholson, D., Stanley, R. H., and Doney, S. C. The triple oxygen isotope tracer of primary productivity in a dynamic ocean model. Global Biogeochem. Cy., 28(5):538-552, 2014. 
Nicholson, D. P., Emerson, S. R., Khatiwala, S., and Hamme, R. C. An inverse approach to estimate bubble-mediated air-sea gas flux from inert gas measurements. In Proceedings of the 6th International Symposium on Gas Transfer at Water Surfaces, Kyoto University Press, Kyoto, Japan, 2011.

Nicholson, D. P., Stanley, R. H. R., Barkan, E., Karl, D. M., Luz, B., Quay, P. D., and Doney, S. C. Evaluating triple oxygen isotope estimates of gross primary production at the Hawaii Ocean Time-series and Bermuda Atlantic Time-series Study sites. J. Geophys. Res. Oceans, 117(C5), 2012.

Nicholson, D. P., Wilson, S. T., Doney, S. C., and Karl, D. M. Quantifying subtropical North Pacific gyre mixed layer primary productivity from Seaglider observations of diel oxygen cycles. Geophys. Res. Lett., 42(10):4032-4039, 2015. doi: 10.1002/2015GL063065. 2015GL063065.

Nieto, K., Demarcq, H., and McClatchie, S. Mesoscale frontal structures in the Canary Upwelling System: New front and filament detection algorithms applied to spatial and temporal patterns. Remote Sens. Environ., 123:339-346, 2012.

Nightingale, P. D., Liss, P. S., and Schlosser, P. Measurements of air-sea gas transfer during an open ocean algal bloom. Geophys. Res. Lett., 27(14):2117-2120, 2000.

NNERRS. NOAA National Estuarine Research Reserve System, System-wide Monitoring Program, Centralized Data Management Office website. http://www.nerrsdata.org, 2016. Accessed January 1, 2016.

Nozais, C., Perissinotto, R., and Tita, G. Seasonal dynamics of meiofauna in a South African temporarily open/closed estuary (Mdloti Estuary, Indian Ocean). Est. Coast. Shelf Sci., 62(1):325-338, 2005.

Olivieri, R. A. and Chavez, F. P. A model of plankton dynamics for the coastal upwelling system of Monterey Bay, California. Deep-Sea Res. Pt. II, 47(5):1077-1106, 2000.

Olivieri, R. A. Plankton dynamics and the fate of primary production in the coastal upwelling ecosystem of Monterey Bay, California. University of California, Santa Cruz, 1996.

O'Neil, J. R. Hydrogen and oxygen isotope fractionation between ice and water. J. Phys. Chem., 72(10):3683-3684, 1968.

Oram, J. J., McWilliams, J. C., and Stolzenbach, K. D. Gradient-based edge detection and feature classification of sea-surface images of the Southern California Bight. Remote Sens. Environ., 112(5):2397-2415, 2008.

Ortiz-Suslow, D. G., Haus, B. K., Williams, N. J., Laxague, N. J. M., Reniers, A. J. H. M., and Graber, H. C. The spatial-temporal variability of air-sea momentum fluxes observed at a tidal inlet. J. Geophys. Res. Oceans, 120(2):660-676, 2015.

Overeem, I., Anderson, R. S., Wobus, C. W., Clow, G. D., Urban, F. E., and Matell, N. Sea ice loss enhances wave action at the Arctic coast. Geophys. Res. Lett., 38(17), 2011. doi: 10.1029/2011GL048681. L17503. 
Palevsky, H. I., Quay, P. D., Lockwood, D. E., and Nicholson, D. P. The annual cycle of gross primary production, net community production, and export efficiency across the North Pacific Ocean. Global Biogeochem. Cy., 30(2):361-380, 2016a. doi: 10.1002/ 2015 GB005318.

Palevsky, H. I., Quay, P. D., and Nicholson, D. P. Discrepant estimates of primary and export production from satellite algorithms, a biogeochemical model, and geochemical tracer measurements in the North Pacific Ocean. Geophys. Res. Lett., 43(16):8645-8653, 2016b.

Papakyriakou, T. and Miller, L. Springtime $\mathrm{CO}_{2}$ exchange over seasonal sea ice in the Canadian Arctic Archipelago. Ann. Glaciol., 52(57):215-224, 2011.

Parker, M., Westhead, M., Doherty, P., and Naug, J. Ecosystem Overview and Assessment Report for the Bras d'Or Lakes, Nova Scotia. Technical Report 2789, Fisheries and Oceans Canada, 2007.

Parmentier, F.-J. W., Christensen, T. R., Sørensen, L. L., Rysgaard, S., McGuire, A. D., Miller, P. A., and Walker, D. A. The impact of lower sea-ice extent on Arctic greenhousegas exchange. Nature Clim. Change, 3(3):195-202, 2013.

Pei, S. and Laws, E. A. Does the ${ }^{14} \mathrm{C}$ method estimate net photosynthesis? Implications from batch and continuous culture studies of marine phytoplankton. Deep-Sea Res. Pt. I, 82:1-9, 2013.

Pennington, J. T., Blum, M., and Chavez, F. P. Seawater sampling by an autonomous underwater vehicle: Gulper sample validation for nitrate, chlorophyll, phytoplankton, and primary production. Limnol. Oceanogr. Meth., 2015.

Pennington, J. T. and Chavez, F. P. Seasonal fluctuations of temperature, salinity, nitrate, chlorophyll and primary production at station H3/M1 over 1989-1996 in Monterey Bay, California. Deep-Sea Res. Pt. II, 47(5):947-973, 2000.

Pennington, J. T., Mahoney, K. L., Kuwahara, V. S., Kolber, D. D., Calienes, R., and Chavez, F. P. Primary production in the eastern tropical Pacific: A review. Prog. Oceanogr., 69(2):285-317, 2006.

Peterson, W. T., Arcos, D. F., McManus, G. B., Dam, H., Bellantoni, D., Johnson, T., and Tiselius, P. The nearshore zone during coastal upwelling: daily variability and coupling between primary and secondary production off central Chile. Prog. Oceanogr., 20(1):1-40, 1988 .

Petrie, B. and Bugden, G. The physical oceanography of the Bras d'Or Lakes. Proc. N. S. Inst. Sci., 42(1), 2002.

Petrie, B. and Raymond, J. The oceanography of the Bras d'Or Lakes: General introduction. Proc. N. S. Inst. Sci., 42(1), 2002.

Pilskaln, C. H., Paduan, J. B., Chavez, F. P., Anderson, R. Y., and Berelson, W. M. Carbon export and regeneration in the coastal upwelling system of Monterey Bay, central California. J. Mar. Res., 54(6):1149-1178, 1996. 
Plant, J. N., Johnson, K. S., Sakamoto, C. M., Jannasch, H. W., Coletti, L. J., Riser, S. C., and Swift, D. D. Net community production at Ocean Station Papa observed with nitrate and oxygen sensors on profiling floats. Global Biogeochem. Cy., 30(6):859-879, 2016. doi: 10.1002/2015GB005349.

Porcelli, D., Ballentine, C. J., and Wieler, R. An overview of noble gas geochemistry and cosmochemistry. Rev. Mineral. Geochem., 47(1):1-19, 2002.

Pratt, K. A., Mayer, J. E., Holecek, J. C., Moffet, R. C., Sanchez, R. O., Rebotier, T. P., Furutani, H., Gonin, M., Fuhrer, K., Su, Y., Guazzotti, S., and Prather, K. A. Development and characterization of an aircraft aerosol time-of-flight mass spectrometer. Anal. Chem, 81:1792-1800, 2009.

Price, J. F., Weller, R. A., and Pinkel, R. Diurnal cycling: Observations and models of the upper ocean response to diurnal heating, cooling, and wind mixing. J. Geophys. Res. Oceans, 91(C7):8411-8427, 1986.

Prokopenko, M. G., Pauluis, O. M., Granger, J., and Yeung, L. Y. Exact evaluation of gross photosynthetic production from the oxygen triple-isotope composition of $\mathrm{O}_{2}$ : Implications for the net-to-gross primary production ratios. Geophys. Res. Lett., 38(14), 2011.

Quay, P. D., Peacock, C., Björkman, K., and Karl, D. M. Measuring primary production rates in the ocean: Enigmatic results between incubation and non-incubation methods at Station ALOHA. Global Biogeochem. Cy., 24(3), 2010. doi: 10.1029/2009GB003665.

Rafelski, L. E., Paplawsky, B., and Keeling, R. F. Continuous measurements of dissolved $\mathrm{O}_{2}$ and oxygen isotopes in the Southern California coastal ocean. Mar. Chem., 174:94-102, 2015.

Redfield, A. C., Ketchum, B., and Richards, F. The influence of organisms on the composition of sea-water. In Hill, M., editor, The Sea: Ideas and Observations of Progress in the Study of the Seas. Volume 2, The Composition of Sea-Water, volume 2, pages 26-77. John Wiley, 1963.

Reuer, M., Barnett, B., Bender, M., Falkowski, P., and Hendricks, M. New estimates of Southern Ocean biological production rates from $\mathrm{O}_{2} / \mathrm{Ar}$ ratios and the triple isotope composition of $\mathrm{O}_{2}$. Deep-Sea Res. Pt. I, 54:951-974, 2007.

Roberts, J. and Roberts, T. D. Use of the Butterworth low-pass filter for oceanographic data. J. Geophys. Res. Oceans, 83(C11):5510-5514, 1978. doi: 10.1029/JC083iC11p05510.

Rose, J. M. and Caron, D. A. Does low temperature constrain the growth rates of heterotrophic protists? Evidence and implications for algal blooms in cold waters. Limnol. Oceanogr., 52(2):886-895, 2007. doi: 10.4319/lo.2007.52.2.0886.

Rosenfeld, L. K., Schwing, F. B., Garfield, N., and Tracy, D. E. Bifurcated flow from an upwelling center: a cold water source for Monterey Bay. Cont. Shelf Res., 14(9):931-964, 1994.

Rutgers van der Loeff, M. M., Cassar, N., Nicolaus, M., Rabe, B., and Stimac, I. The influence of sea ice cover on air-sea gas exchange estimated with radon-222 profiles. J. Geophys. Res. Oceans, 119(5):2735-2751, 2014. doi: 10.1002/2013JC009321. 
Ryan, J. P., Fischer, A. M., Kudela, R. M., Gower, J. F., King, S. A., Marin, R., and Chavez, F. P. Influences of upwelling and downwelling winds on red tide bloom dynamics in Monterey Bay, California. Cont. Shelf Res., 29(5):785-795, 2009.

Sabine, C. L., Feely, R. A., Gruber, N., Key, R. M., Lee, K., Bullister, J. L., Wanninkhof, R., Wong, C., Wallace, D. W., Tilbrook, B., et al. The oceanic sink for anthropogenic $\mathrm{CO}_{2}$. Science, 305(5682):367-371, 2004.

Sakamoto, C. M., Friederich, G. E., and Codispoti, L. A. MBARI procedures for automated nutrient analyses using a modified Alpkem Series 300 rapid flow analyzer. Technical report, Monterey Bay Aquarium Research Institute, 1990.

Salonen, K., Leppäranta, M., Viljanen, M., and Gulati, R. D. Perspectives in winter limnology: closing the annual cycle of freezing lakes. Aquat. Ecol., 43(3):609-616, 2009.

Sano, Y. and Takahata, N. Measurement of noble gas solubility in seawater using a quadrupole mass spectrometer. J. Oceanogr., 61(3):465-473, 2005.

Santoro, A. E., Sakamoto, C. M., Smith, J. M., Plant, J. N., Gehman, A. L., Worden, A. Z., Johnson, K. S., Francis, C. A., and Casciotti, K. L. Measurements of nitrite production in and around the primary nitrite maximum in the central California Current. Biogeosciences, 10(11):7395-7410, 2013.

Sarma, V. V. S. S., Abe, O., Hashimoto, S., Hinuma, A., and Saino, T. Seasonal variations in triple oxygen isotopes and gross oxygen production in the Sagami Bay, central Japan. Limnol. Oceanogr., 50(2):544-552, 2005.

Sarma, V. V. S. S., Abe, O., Honda, M., and Saino, T. Estimating of gas transfer velocity using triple isotopes of dissolved oxygen. J. Oceanogr., 66(4):505-512, 2010.

Sarmiento, J. L. and Gruber, N. Ocean Biogeochemical Dynamics. Princeton University Press, Princeton, NJ, USA, 2006.

Saucier, F., Senneville, S., Prinsenberg, S., Roy, F., Smith, G., Gachon, P., Caya, D., and Laprise, R. Modelling the sea ice-ocean seasonal cycle in Hudson Bay, Foxe Basin and Hudson Strait, Canada. Clim. Dynam., 23(3-4):303-326, 2004.

Schmidt, G., Bigg, G. R., and Rohling, E. J. Global Seawater Oxygen-18 Database - v1.21. http://data.giss.nasa.gov/o18data/, 1999. Accessed September 1, 2016.

Schmidt, G. A. Forward modeling of carbonate proxy data from planktonic foraminifera using oxygen isotope tracers in a global ocean model. Paleoceanography, 14(4):482-497, 1999. doi: 10.1029/1999PA900025.

Seager, R., Hoerling, M., Schubert, S., Wang, H., Lyon, B., Kumar, A., Nakamura, J., and Henderson, N. Causes of the 2011-14 California drought. J. Climate, 28(18):6997-7024, 2015 .

Severinghaus, J. P., Grachev, A., Luz, B., and Caillon, N. A method for precise measurement of argon 40/36 and krypton/argon ratios in trapped air in polar ice with applications to past firn thickness and abrupt climate change in Greenland and at Siple Dome, Antarctica. Geochim. Cosmochim. Acta, 67(3):325-343, 2003. doi: 10.1016/S0016-7037(02)00965-1. 
Siegel, D. A., Buesseler, K. O., Behrenfeld, M. J., Benitez-Nelson, C. R., Boss, E., Brzezinski, M. A., Burd, A., Carlson, C. A., D'Asaro, E. A., Doney, S. C., et al. Prediction of the export and fate of global ocean net primary production: The EXPORTS Science Plan. Front. Mar. Sci., 3:22, 2016.

Sigman, D. M., Casciotti, K. L., Andreani, M., Barford, C., Galanter, M., and Böhlke, J. K. A bacterial method for the nitrogen isotopic analysis of nitrate in seawater and freshwater. Anal. Chem., 73(17):4145-4153, 2001.

Sigman, D. M. and Boyle, E. A. Glacial/interglacial variations in atmospheric carbon dioxide. Nature, 407(6806):859-869, 2000. doi: 10.1038/35038000.

Smith, D. C. and Morison, J. H. A numerical study of haline convection beneath leads in sea ice. J. Geophys. Res. Oceans, 98(C6):10069-10083, 1993.

Smith, J. M., Casciotti, K. L., Chavez, F. P., and Francis, C. A. Differential contributions of archaeal ammonia oxidizer ecotypes to nitrification in coastal surface waters. ISME J., 8(8):1704-1714, 2014a.

Smith, J. M., Chavez, F. P., and Francis, C. A. Ammonium uptake by phytoplankton regulates nitrification in the sunlit ocean. PLOS ONE, 9(9):e108173, 2014b.

Smith, J. M., Damashek, J., Chavez, F. P., and Francis, C. A. Factors influencing nitrification rates and the abundance and transcriptional activity of ammonia-oxidizing microorganisms in the dark northeast Pacific Ocean. Limnol. Oceanogr., 61(2):596-609, 2016. doi: 10.1002/lno.10235.

Smith, R. E. H., Geider, R. J., and Platt, T. Microplankton productivity in the oligotrophic ocean. Nature, 311:252-254, 1984.

Sonnerup, R. E., Mecking, S., and Bullister, J. L. Transit time distributions and oxygen utilization rates in the Northeast Pacific Ocean from chlorofluorocarbons and sulfur hexafluoride. Deep-Sea Res. Pt. I, 72:61-71, 2013.

Spitzer, W. S. and Jenkins, W. J. Rates of vertical mixing, gas exchange and new production: Estimates from seasonal gas cycles in the upper ocean near Bermuda. J. Mar. Res., 47: 169-196, 1989. doi: 10.1357/002224089785076370.

Squire, V. A., Dugan, J. P., Wadhams, P., Rottier, P. J., and Liu, A. K. Of ocean waves and sea ice. Ann. Rev. Fluid Mech., 27(1):115-168, 1995.

St. Laurent, L. and Schmitt, R. W. The contribution of salt fingers to vertical mixing in the North Atlantic Tracer Release Experiment. J. Phys. Oceanogr., 29(7):1404-1424, 1999.

Stanley, R. H. R., Kirkpatrick, J. B., Cassar, N., Barnett, B. A., and Bender, M. L. Net community production and gross primary production rates in the western equatorial Pacific. Global Biogeochem. Cy., 24(4), 2010. doi: 10.1029/2009GB003651. GB4001.

Stanley, R. H. R. and Jenkins, W. J. Noble gases in seawater as tracers for physical and biogeochemical ocean processes. In The Noble Gases as Geochemical Tracers, pages 55-79. Springer, 2013. 
Stanley, R. H. R., Jenkins, W. J., and Doney, S. C. Quantifying seasonal air-sea gas exchange processes using noble gas time-series: A design experiment. J. Mar. Res., 64(2):267-295, 2006.

Stanley, R. H. R., Baschek, B., Lott, D. E., and Jenkins, W. J. A new automated method for measuring noble gases and their isotopic ratios in water samples. Geochem. Geophys. Geosyst., 10(5), 2009a. doi: 10.1029/2009GC002429.

Stanley, R. H. R., Jenkins, W. J., Lott, D. E., and Doney, S. C. Noble gas constraints on air-sea gas exchange and bubble fluxes. J. Geophys. Res. Oceans, 114(C11), 2009b. doi: 10.1029/2009JC005396.

Stanley, R. H. R., Sandwith, Z. O., and Williams, W. J. Rates of summertime biological productivity in the Beaufort Gyre: A comparison between the low and record-low ice conditions of August 2011 and 2012. J. Mar. Sys., 147:29-44, 2015.

Steemann Nielsen, E. Measurement of the production of organic matter in the sea by means of carbon-14. Nature, 167:684-685, 1951.

Steemann Nielsen, E. The use of radio-active carbon $\left(\mathrm{C}^{14}\right)$ for measuring organic production in the sea. J. Cons., 18(2):117-140, 1952.

Steemann Nielsen, E. and Jensen, E. A. Primary oceanic production. Galathea Rep., 1: 49-135, 1957.

Steig, E. J., Gkinis, V., Schauer, A. J., Schoenemann, S. W., Samek, K., Hoffnagle, J., Dennis, K. J., and Tan, S. M. Calibrated high-precision ${ }^{17} \mathrm{O}$-excess measurements using cavity ring-down spectroscopy with laser-current-tuned cavity resonance. Atmos. Meas. Tech., 7(8):2421-2435, 2014.

Steinberg, D. K., Carlson, C. A., Bates, N. R., Goldthwait, S. A., Madin, L. P., and Michaels, A. F. Zooplankton vertical migration and the active transport of dissolved organic and inorganic carbon in the Sargasso Sea. Deep-Sea Res. Pt. I, 47(1):137-158, 2000.

Strain, P. M. Nutrient, dissolved oxygen, trace metal and related measurements in the Bras d'Or Lakes, 1995-97. Can. Data Rep. Fish Aquat. Sci., 1073:56 pp., 2001.

Strain, P. M. and Yeats, P. A. The chemical oceanography of the Bras d'Or Lakes. Proc. N. S. Inst. Sci., 42(1):37-64, 2002.

Stute, M. and Schlosser, P. Principles and applications of the noble gas paleothermometer. Climate Change in Continental Isotopic Records, pages 89-100, 1993. doi: 10.1029/GM078p0089.

Sullivan, P. P., Romero, L., McWilliams, J. C., and Melville, W. K. Transient evolution of langmuir turbulence in ocean boundary layers driven by hurricane winds and waves. $J$. Phys. Oceanogr., 42(11):1959-1980, 2012.

Surge, D. M. and Lohmann, K. C. Temporal and spatial differences in salinity and water chemistry in SW Florida estuaries: effects of human-impacted watersheds. Estuaries, 25 (3):393-408, 2002. 
Sweeney, C., Gloor, E., Jacobson, A. R., Key, R. M., McKinley, G., Sarmiento, J. L., and Wanninkhof, R. Constraining global air-sea gas exchange for $\mathrm{CO}_{2}$ with recent bomb ${ }^{14}$ C measurements. Global Biogeochem. Cy., 21(2), 2007. doi: 10.1029/2006GB002784. GB2015.

Swets, D. E., Lee, R. W., and Frank, R. C. Diffusion coefficients of helium in fused quartz. J. Chem. Phys., 34(1):17-22, 1961.

Takahashi, T., Sutherland, S. C., Wanninkhof, R., Sweeney, C., Feely, R. A., Chipman, D. W., Hales, B., Friederich, G., Chavez, F., Sabine, C., et al. Climatological mean and decadal change in surface ocean $\mathrm{pCO}_{2}$, and net sea-air $\mathrm{CO}_{2}$ flux over the global oceans. Deep-Sea Res. Pt. II, 56(8):554-577, 2009.

Tan, F. C. and Strain, P. M. The distribution of sea ice meltwater in the eastern Canadian Arctic. J. Geophys. Res. Oceans, 85(C4):1925-1932, 1980.

Teeter, L. Modelling oxygen and argon to improve estimation of net community productivity in a coastal upwelling zone using $\Delta \mathrm{O}_{2} /$ Ar. Master's thesis, University of Victoria, Victoria, BC, Canada, 2014.

Thiemens, M. H., Jackson, T., Zipf, E. C., Erdman, P. W., and van Egmond, C. Carbon dioxide and oxygen isotope anomalies in the mesosphere and stratosphere. Science, 270 (5238):969-972, 1995. doi: 10.1126/science.270.5238.969.

Thompson, A. F., Lazar, A., Buckingham, C., Naveira Garabato, A. C., Damerell, G. M., and Heywood, K. J. Open-ocean submesoscale motions: A full seasonal cycle of mixed layer instabilities from gliders. J. Phys. Oceanogr., 46(4):1285-1307, 2016.

Timsic, S. and Patterson, W. P. Spatial variability in stable isotope values of surface waters of Eastern Canada and New England. J. Hydrol., 511:594-604, 2014.

Top, Z., Martin, S., and Becker, P. A laboratory study of dissolved noble gas anomaly due to ice formation. Geophys. Res. Lett., 15(8):796-799, 1988.

Tortell, P. D. Dissolved gas measurements in oceanic waters made by membrane inlet mass spectrometry. Limnol. Oceanogr. Methods, 3:24-37, 2005.

Tortell, P. D. and Long, M. C. Spatial and temporal variability of biogenic gases during the southern ocean spring bloom. Geophys. Res. Lett., 36(1), 2009. doi: 10.1029/ 2008GL035819.

Tortell, P. D., Asher, E. C., Ducklow, H. W., Goldman, J. A., Dacey, J. W., Grzymski, J. J., Young, J. N., Kranz, S. A., Bernard, K. S., and Morel, F. M. Metabolic balance of coastal Antarctic waters revealed by autonomous $\mathrm{pCO}_{2}$ and $\Delta \mathrm{O}_{2} /$ Ar measurements. Geophys. Res. Lett., 41(19):6803-6810, 2014.

Twiss, M. R., McKay, R. M. L., Bourbonniere, R. A., Bullerjahn, G. S., Carrick, H. J., Smith, R. E. H., Winter, J. G., D'souza, N. A., Furey, P. C., Lashaway, A. R., et al. Diatoms abound in ice-covered Lake Erie: An investigation of offshore winter limnology in Lake Erie over the period 2007 to 2010. J. Great Lakes Res., 38(1):18-30, 2012.

Upstill-Goddard, R. C. Air-sea gas exchange in the coastal zone. Estuar. Coast. Shelf Sci., 70(3):388-404, 2006. 
Vachon, R. W., White, J. W. C., Gutmann, E., and Welker, J. M. Amount-weighted annual isotopic $\left(\delta^{18} \mathrm{O}\right)$ values are affected by the seasonality of precipitation: A sensitivity study. Geophys. Res. Lett., 34(21), 2007. doi: 10.1029/2007GL030547. L21707.

Vaughan, D. G., Comiso, J. C., Allison, I., Carrasco, J., Kaser, G., Kwok, R., Mote, P., Murray, T., Paul, F., Ren, J., Rignot, E., Solomina, O., Steffen, K., and Zhang, T. Observations: Cryosphere. In Stocker, T. F., Qin, D., Plattner, G.-K., Tignor, M., Allen, S. K., Boschung, J., Nauels, A., Xia, Y., Bex, V., and Midgley, P., editors, Climate Change 2013: The Physical Science Basis. Contribution of Working Group I to the Fifth Assessment Report of the Intergovernmental Panel on Climate Change, chapter 4, pages 317-482. Cambridge University Press, Cambridge, United Kingdom and New York, NY, USA, 2013.

Virkki, V. T., Ketola, R. A., Ojala, M., Kotiaho, T., Komppa, V., Grove, A., and Facchetti, S. On-site environmental analysis by membrane inlet mass spectrometry. Anal. Chem., 67(8):1421-1425, 1995.

Visser, A., Singleton, M. J., Hillegonds, D. J., Velsko, C. A., Moran, J. E., and Esser, B. K. A membrane inlet mass spectrometry system for noble gases at natural abundances in gas and water samples. Rapid Commun. Mass Spectrom., 27(21):2472-2482, 2013.

Volpe, A. M., Esser, B. K., and Bianchini, G. M. Real-time ocean chemical measurement: at-sea ICP-MS experiments. Journal of Analytical Atomic Spectrometry, 16(8):801-805, 2001.

Wang, R., Zhang, H. Y., Feron, P. H. M., and Liang, D. T. Influence of membrane wetting on $\mathrm{CO}_{2}$ capture in microporous hollow fiber membrane contactors. Sep. Purif. Technol., 46(1):33-40, 2005.

Wankel, S. D., Kendall, C., Francis, C. A., and Paytan, A. Nitrogen sources and cycling in the San Francisco Bay Estuary: A nitrate dual isotopic composition approach. Limnol. Oceanogr., 51(4):1654-1664, 2006.

Wanninkhof, R. Relationship between wind speed and gas exchange over the ocean revisited. Limnol. Oceanogr. Meth., 12(6):351-362, 2014.

Wanninkhof, R. and McGillis, W. R. A cubic relationship between air-sea $\mathrm{CO}_{2}$ exchange and wind speed. Geophys. Res. Lett., 26(13):1889-1892, 1999.

Wanninkhof, R., Ledwell, J. R., Broecker, W. S., and Hamilton, M. Gas exchange on Mono Lake and Crowley Lake, California. J. Geophys. Res. Oceans, 92(C13):14567-14580, 1987.

Wanninkhof, R., Asher, W., Weppernig, R., Chen, H., Schlosser, P., Langdon, C., and Sambrotto, R. Gas transfer experiment on Georges Bank using two volatile deliberate tracers. J. Geophys. Res., 98(C11):20237-20248, 1993.

Wanninkhof, R., Asher, W. E., Ho, D. T., Sweeney, C., and McGillis, W. R. Advances in quantifying air-sea gas exchange and environmental forcing. Ann. Rev. Mar. Sci., 1: 213-244, 2009.

Wanninkhof, R., Park, G.-H., Takahashi, T., Sweeney, C., Feely, R. A., Nojiri, Y., Gruber, N., Doney, S. C., McKinley, G. A., Lenton, A., et al. Global ocean carbon uptake: 
magnitude, variability and trends. Biogeosciences, 10:1983-2000, 2013. doi: 10.5194/ bg-10-1983-2013.

Ward, B. B. Temporal variability in nitrification rates and related biogeochemical factors in Monterey Bay, California, USA. Mar. Ecol. Prog. Ser., 292(97):109, 2005.

Watson, A., Liss, P., and Duce, R. Design of a small-scale in situ iron fertilization experiment. Limnol. Oceanogr., 36(8):1960-1965, 1991a.

Watson, A. J., Upstill-Goddard, R. C., and Liss, P. S. Air-sea gas exchange in rough and stormy seas measured by a dual-tracer technique. Nature, 349(6305):145-147, 1991b.

Weeding, B. and Trull, T. W. Hourly oxygen and total gas tension measurements at the Southern Ocean Time Series site reveal winter ventilation and spring net community production. J. Geophys. Res. Oceans, 119(1):348-358, 2014.

Weeks, W. F. and Ackley, S. F. The growth, structure, and properties of sea ice. In Untersteiner, N., editor, The Geophysics of Sea Ice, pages 9-164. Springer US, Boston, MA, 1986. doi: 10.1007/978-1-4899-5352-0_2.

Weiss, R. F. Solubility of helium and neon in water and seawater. J. Chem. Eng. Data, 16 (2):235-241, 1971.

Weiss, R. F. and Kyser, T. K. Solubility of krypton in water and sea water. J. Chem. Eng. Data, 23(1):69-72, 1978. doi: 10.1021/je60076a014.

Welch, H. E., Bergmann, M. A., Jorgenson, J. K., and Burton, W. A subice suction corer for sampling epontic ice algae. Can. J. Fish. Aquat. Sci., 45(3):562-568, 1988.

Wiegner, T. N., Seitzinger, S. P., Glibert, P. M., and Bronk, D. A. Bioavailability of dissolved organic nitrogen and carbon from nine rivers in the eastern United States. Aquat. Microb. Ecol., 43(3):277-287, 2006.

Wilkerson, F. P., Dugdale, R. C., Kudela, R. M., and Chavez, F. P. Biomass and productivity in Monterey Bay, California: contribution of the large phytoplankton. Deep-Sea Res. Pt. II, 47(5):1003-1022, 2000.

Williams, P. J. and Purdie, D. A. In vitro and in situ derived rates of gross production, net community production and respiration of oxygen in the oligotrophic subtropical gyre of the North Pacific Ocean. Deep-Sea Res. Pt. A, 38(7):891-910, 1991.

Williams, P. J., Heinemann, K. R., Marra, J., and Purdie, D. A. Comparison of ${ }^{14} \mathrm{C}$ and $\mathrm{O}_{2}$ measurements of phytoplankton production in oligotrophic waters. Nature, pages 49-50, 1983.

Williams, P. J. Chemical and tracer methods of measuring plankton production. ICES Mar. Sci. Symp., 197:20-36, 1993.

Williams, P. J., Morris, P. J., and Karl, D. M. Net community production and metabolic balance at the oligotrophic ocean site, station ALOHA. Deep-Sea Res. Pt. I, 51(11): 1563-1578, 2004. 
Wilson, S. T., Barone, B., Ascani, F., Bidigare, R. R., Church, M. J., del Valle, D. A., Dyhrman, S. T., Ferrón, S., Fitzsimmons, J. N., Juranek, L. W., Kolber, Z. S., Letelier, R. M., Martínez-García, S., Nicholson, D. P., Richards, K. J., Rii, Y. M., Rouco, M., Viviani, D. A., White, A. E., Zehr, J. P., and Karl, D. M. Short-term variability in euphotic zone biogeochemistry and primary productivity at Station ALOHA: A case study of summer 2012. Global Biogeochem. Cy., 29(8):1145-1164, 2015.

Wolk, F., Yamazaki, H., Seuront, L., and Lueck, R. G. A new free-fall profiler for measuring biophysical microstructure. J. Atm. Ocean. Tech., 19(5):780-793, 2002.

Wong, P. P., Losada, I. J., Gattuso, J., Hinkel, J., Khattabi, A., McInnes, K., Saito, Y., and Sallenger, A. Coastal systems and low-lying areas. In Field, C. B., Barros, V. R., Dokken, D. J., Mach, K. J., Mastrandrea, M. D., Bilir, T. E., Chatterjee, M., Ebi, K. L., Estrada, Y. O., Genova, R. C., Girma, B., Kissel, E. S., Levy, A. N., MacCracken, S., Mastrandrea, P. R., and L.White, L., editors, Climate Change 2014: Impacts, Adaptation, and Vulnerability. Part A: Global and Sectoral Aspects. Contribution of Working Group II to the Fifth Assessment Report of the Intergovernmental Panel on Climate Change, chapter 5, pages 361-409. Cambridge University Press, Cambridge, United Kingdom and New York, NY, USA, 2014.

Wood, D. and Caputi, R. Solubilities of Kr and Xe in fresh and sea water. Technical report, U.S. Naval Radiological Defense Laboratory, San Francisco, CA, U.S. Naval Radiological Defense Laboratory, San Francisco, CA, 1966.

Woodson, C., Washburn, L., Barth, J. A., Hoover, D., Kirincich, A. R., McManus, M., Ryan, J. P., and Tyburczy, J. Northern Monterey Bay upwelling shadow front: Observations of a coastally and surface-trapped buoyant plume. J. Geophys. Res. Oceans, 114(C12), 2009. doi: 10.1029/2009JC005623. C12013.

Wüest, A., Van Senden, D., Imberger, J., Piepke, G., and Gloor, M. Comparison of diapycnal diffusivity measured by tracer and microstructure techniques. Dynam. Atmos. Ocean, 24 (1):27-39, 1996.

Wurgaft, E., Shamir, O., and Angert, A. Technical Note: The effect of vertical turbulent mixing on gross $\mathrm{O}_{2}$ production assessments by the triple isotopic composition of dissolved $\mathrm{O}_{2}$. Biogeosciences, 10(12):8363-8371, 2013.

Yang, B., Sheng, J., Hatcher, B. G., and Petrie, B. Numerical study of circulation and temperature-salinity distributions in the Bras d'Or Lakes. Ocean Dyn., 57(4-5):245-268, 2007.

Yool, A., Martin, A. P., Fernández, C., and Clark, D. R. The significance of nitrification for oceanic new production. Nature, 447(7147):999-1002, 2007.

Zhou, J., Delille, B., Eicken, H., Vancoppenolle, M., Brabant, F., Carnat, G., Geilfus, N.-X., Papakyriakou, T., Heinesch, B., and Tison, J.-L. Physical and biogeochemical properties in landfast sea ice (barrow, alaska): Insights on brine and gas dynamics across seasons. J. Geophys. Res. Oceans, 118(6):3172-3189, 2013. doi: 10.1002/jgrc.20232.

Zhou, J., Delille, B., Brabant, F., and Tison, J.-L. Insights into oxygen transport and net community production in sea ice from oxygen, nitrogen and argon concentrations. Biogeosciences, 11(18):5007-5020, 2014. 